\title{
On polyhedral cones of cooperative games
}

\author{
Citation for published version (APA):
}

Derks, J. J. M. (1991). On polyhedral cones of cooperative games. [Doctoral Thesis, Maastricht University]. Datawyse. https://doi.org/10.26481/dis.19911212jd

Document status and date:

Published: 01/01/1991

DOI:

10.26481/dis.19911212jd

Document Version:

Publisher's PDF, also known as Version of record

\section{Please check the document version of this publication:}

- A submitted manuscript is the version of the article upon submission and before peer-review. There can be important differences between the submitted version and the official published version of record.

People interested in the research are advised to contact the author for the final version of the publication, or visit the DOI to the publisher's website.

- The final author version and the galley proof are versions of the publication after peer review.

- The final published version features the final layout of the paper including the volume, issue and page numbers.

Link to publication

\footnotetext{
General rights rights.

- You may freely distribute the URL identifying the publication in the public portal. please follow below link for the End User Agreement:

www.umlib.nl/taverne-license

Take down policy

If you believe that this document breaches copyright please contact us at:

repository@maastrichtuniversity.nl

providing details and we will investigate your claim.
}

Copyright and moral rights for the publications made accessible in the public portal are retained by the authors and/or other copyright owners and it is a condition of accessing publications that users recognise and abide by the legal requirements associated with these

- Users may download and print one copy of any publication from the public portal for the purpose of private study or research.

- You may not further distribute the material or use it for any profit-making activity or commercial gain

If the publication is distributed under the terms of Article $25 \mathrm{fa}$ of the Dutch Copyright Act, indicated by the "Taverne" license above, 
ON POLYHEDRAL CONES

OF

COOPERATIVE GAMES 



\section{ON POLYHEDRAL CONES \\ OF \\ COOPERATIVE GAMES}

\section{PROEFSCHRIFT}

ter verkrijging van de graad van doctor

aan de Rijksuniversiteit Limburg te Maastricht, op gezag van de Rector Magnificus, Prof. Mr. M.J. Cohen, volgens het besluit van het College van Dekanen, in het openbaar te verdedigen op

donderdag 12 december 1991 om 16.00 uur

door

\section{Jean Joseph Marie Derks}

geboren te Roermond op 9 april 1956 


\section{Promotores:}

Prof.dr.ir.drs. O.J. Vrieze

Prof.dr. S.H. Tijs (KU Brabant)

Co-promotor:

Dr. H.J.M. Peters

Beoordelingscommissie:

Prof.dr. A.W.J. Kolen (voorzitter)

Dr. Y. Crama

Dr. T.S.H. Driessen (Universiteit Twente)

Prof.dr. H.Th. Jongen (RWTH Aken, BRD)

Dr. J.A.M. Potters (KU Nijmegen)

Druk: Datawyse Maastricht/Krips Repro Meppel

CIP-DATA KONINKLIJKE BIBLIOTHEEK, DEN HAAG

Derks, Jean Joseph Marie

On polyhedral cones of cooperative games / Jean Joseph

Marie Derks. - Maastricht : Datawyse.

Thesis Maastricht. - With index, ref. - With a summary

in Dutch.

ISBN 90-5291-069-3

NUGI 811

Subject heading: cooperative game theory. 


\section{Contents}

Preface $\quad$ ix

Introduction ................... ix

An outline of the monograph $\ldots \ldots \ldots$ xi

Acknowledgements .................... . . xii

Notations and symbols xiii

1 Preliminaries 1

1.1 Introduction . . . . . . . . . . . . . . . 1

1.2 Cooperative games . . . . . . . . . . . . . . . 1

1.2.1 The game model . . . . . . . . . . . . . . 1

1.2.2 The core and the domination core . . . . . . . 5

1.2 .3 Core catchers . . . . . . . . . . . . . . . 9 9

1.2.4 Single-valued solution schemes . . . . . . . . 16

1.2.5 Summary of the introduced classes of garnes . . . . . 18

1.2.6 Games arising from cost situations . . . . . . . . . 18

1.3 Pollyhedral cones . . . . . . . . . . . . . . . . 20

1.3 .1 Cones . . . . . . . . . . . . . . . . . 20

1.3.2 Extreme directions . . . . . . . . . . . . 22

1.3 .3 Cones of games . . . . . . . . . . . . 25

2 Cones of games with a non-empty core 33

2.1 Introduction . . . . . . . . . . . . . . 33

2.2 Cones of games with a non-empty core . . . . . . . . 35

2.2.1 The cone of games with a non-empty core . . . . . 35

2.2.2 A proof of the Bondareva-Shapley correspondence . . 40

2.2.3 The cone of non-negative games with a non-empty core 41

2.2.4 Constructive methods . . . . . . . . . . . 45

2.2.5 Population monotonic allocation schemes ...... 50 
2.2.6 The airport cost savings games ... . . . . . 52

2.3 Linear programming games ................ 55

2.3.1 Linear production games ........... 56

2.3.2 Balanced linear programming games . . . . . . . 59

2.3.3 Core allocations of linear programming games . . . . 63

2.4 Multicommodity games ................ 64

2.4.1 Balanced multicommodity games ......... 65

2.4.2 Multicommodity core allocations . . . . . . . 66

2.4.3 The quasi-additive multicommodity games . . . . . . 68

2.5 Flow games . . . . . . . . . . . . . . . 70

2.5.1 A multicommodity flow model . . . . . . . 70

2.5.2 The flow game . . . . . . . . . . . . 74

2.5.3 Multicommodity games arising from veto-controlled flow models . . . . . . . . . . . . . . . 76

2.5 .4 Concluding remarks ............. 81

3 Cones of semibalanced games and related classes 83

3.1 Introduction . . . . . . . . . . . . . . . 83

3.2 Cones of semibalanced games . . . . . . . . . . . 84

3.2.1 The cone of semibalanced games ... . . . . . . 84

3.2.2 The cone of non-negative semibalanced games . . . . 87

3.3 Cones of maximally blocked games . . . . . . . . . . . . 90

3.3.1 The cones of maximally blocked games and 1-convex games .................... 90

3.3.2 Cones of non-negative maximally blocked games . . 9 93

3.4 The class of semiconvex games . . . . . . . . . . . 97

3.4.1 A decomposition of the cone of semiconvex games . 98

3.5 Cones of quasi-balanced games . . . . . . . . . . 103

3.5.1 The cone of 0-normalized quasi-balanced games . . . 104

3.5.2 The cone of quasi-balanced games . . . . . . 108

3.5.3 A decomposition procedure . . . . . . . . . 110

3.6 A comparison of considered classes of games . . . . . . . . 112

3.6 .1 A review . . . . . . . . . . . 112

3.6.2 Spanning sets for the game space . . . . . . . 116

3.6.3 The cost games of the considered games . . . . . . 121

4 Minimum closed cones of games $\quad 125$

4.1 Introduction . . . . . . . . . . . . . . . . 125

4.2 Minimum closed cones of games . . . . . . . . . . 126 
4.2.1 A characterization of minimum closed cones . . . . 126

4.2.2 Minimum closed cones of games . . . . . . . . 128

4.2.3 Essential coalitions and minimum covers . . . . . . . 130

4.2 .4 Decomposability . . . . . . . . . . . . . 133

4.2 .5 Extreme games . . . . . . . . . . . . 136

4.3 The cone of non-negative superadditive games . . . . . . 137

4.3.1 Extreme superadditive games ...........137

4.3.2 A survey of results in the literature concerning extreme superadditive games . . . . . . . . . . 141

5 Upper bound sets for the class of superadditive games 145

5.1 Introduction . . . . . . . . . . . . . . 145

5.2 The semistable games . . . . . . . . . . . . 146

5.3 The splitting stable games . . . . . . . . . . . . 150

5.4 The partition stable games $\ldots \ldots \ldots \ldots . \ldots 152$

$\begin{array}{ll}\text { Bibliography } & \mathbf{1 5 7}\end{array}$

$\begin{array}{ll}\text { Author index } & 163\end{array}$

$\begin{array}{lr}\text { Subject index } & 165\end{array}$

$\begin{array}{lr}\text { Samenvatting } & 169\end{array}$

$\begin{array}{lr}\text { Curriculum vitae } & 173\end{array}$ 



\section{Preface}

\section{Introduction}

The main subject of Game Theory is the study of mathematical models in which conflict and cooperation have an explicit meaning. As such it has many applications in management science, social science and economic theory. Game theoretic models have been studied extensively during the last five decades, initiated by the publication of the comprehensive work Theory of Games and Economic Behavior in 1944, written by John von Neumann and Oskar Morgenstern. The first author is generally regarded as the founder of Game Theory.

The game model studied in this monograph is the main subject of Cooperative Game Theory. It consists of two components: a (finite) set of players and a real-valued pay-off function. It is assumed that the players are free to cooperate and that the pay-off function reflects the savings or strength of the coalitions among the players. There are excellent reviews available on this subject. We mention here Luce and Raiffa [1957], Owen [1982], Rosenmüller [1981] and Shubik [1982].

With respect to games of the above type, the two following questions can be posed: which coalitions are likely to be formed in the course of the negotiations, and, secondly, what is the relative strength of a player in a game. The first question regards the description and discussion of processes of cooperative behavior. In this monograph several properties for games are considered which induce or encourage the formation of the grand coalition of all participating players. In the context of the formation of the grand coalition the second question can be rephrased as how to distribute the savings of the grand coalition among the players. A prescription of the distribution of these savings is called a solution scheme. Several schemes are discussed in the literature; all of them are based on different insights of how to describe or define the strength of a player in a game. Furthermore, each 
scheme proposes stable or reasonable distributions only for games fulfilling certain properties, or are defined on a subclass of the game space.

The core is probably the most studied and applied set-valued solution scheme. It consists of allocations representing distributions of the savings of the grand coalition among the players which cannot be improved by one of the other coalitions of players. One generally considers the existence of core allocations as a necessary and sufficient condition to assure the stability of the grand coalition. Not all games, however, possess a non-empty core. Moreover, relatively complex methods, based on linear programming, are needed to compute a core allocation, if at all possible. Partly due to these considerations, various kinds of games have been introduced and studied for which the core has suitable computational properties or for which an alternative computationally simple set of allocations is available containing the core as a subset.

We will consider several types of games which are introduced in the context as described in the previous paragraph. The primary intention is to obtain insight in the structure of the corresponding classes of games considered as subsets in the game space. Also the restrictions to the 0 -normalized games and to the non-negative games are studied for most of the considered classes of games. Especially the latter restriction is a standard limitation of the worth of a coalition if one considers negative savings or profit as having no meaning.

The game space is treated as a Euclidean vector space with dimension equal to the total of non-empty coalitions, in which most of the considered classes may be seen as polyhedral cones, i.e., as a finite intersection of closed half-spaces. According to well-known duality arguments, a polyhedral cone is characterized by the existence of a finite generating set, i.e., a finite set with the property that each element in the cone can be described as a non-negative weighted sum of the elements of this subset. Some of the boundary points of a cone, namely the extreme directions, are contained in each generating set (up to a non-negative multiplication factor).

Besides its mathematical interest the characterization of (the) extreme directions of a class of games and the construction of a (finite) generating set have several interesting game theoretic features. First of all it is relevant to understand the behavior of a solution scheme on the games in a generating set of a particular cone of games in order to describe its behavior on the whole cone. Moreover, if there are shortcomings in the underlying ideas of rationality, these should be especially revealed by the outcomes of the solution scheme with respect to the extreme games. In this context, one 
other remark seems justified: if a solution scheme is seen as the outcome of a certain social behavior and the considered cone of games consists of all those games for which the solution scheme yields a reasonable outcome, then the extremality of a game in the geometrical sense should correspond to a notion of extremality in the social behavior sense (cf. Rosenmüller [1977]). This extremality correspondence will be illustrated by some of the considered classes of games.

\section{An outline of the monograph}

Chapter 1 starts with an introduction of the game theoretic notions which are used throughout. We will discuss game properties such as superadditivity, convexity, balancedness, semibalancedness and quasi-balancedness. Furthermore, we will treat several set-valued solution schemes such as the core, the domination core, the marginal core and the core cover. The latter two schemes may be considered as relaxations of the core concept. Two single-valued solution schemes are discussed: the Shapley value and the $r$ value. Furthermore, several concepts from convex analysis such as polyhedral cones, generating sets and extreme elements are explained in more detail, and some preliminary results are given. Basic knowledge of linear algebra, set theory, and Euclidean topology is sufficient for the understanding of most of the mathematical reasoning.

Chapter 2 is devoted to classes of games for which the core is non-empty. The main result is the characterization of the extreme games of the class of non-negative games with a non-empty core. These games correspond to the $\{0,1\}$-valued games for which so-called veto-players exist. This implies that these games fulfill a notion of extremality of social behavior and, therefore, the core is an example for which the above mentioned extremality correspondence holds. As an application of our main result, alternative proof will be given of the well-known Bondareva-Shapley correspondence expressing the equivalence of the balancedness property and the existence of a non-empty core. Furthermore, several subclasses of games with a non-empty core are considered and identified by a construction of a generating set. We mention here the class of games having a so-called population monotonic allocation scheme and the class of games containing the airport cost savings games. Chapter 2 is concluded by a survey of applications of our main result in situations in which $\{0,1\}$-valued games are used as a way to describe committee control. This will be done for the linear programming games, the 
multicommodity games and the (multicommodity) flow games.

In chapter 3 we will present and discuss generating sets for several classes of games for which the semibalancedness property holds. Besides the semibalanced games, we will discuss the 1-convex games (games for which the non-empty core equals the marginal core) and related games such as the maximally blocked games and the clan games. The semiconvex games and the quasi-balanced games (games with non-empty core cover) are also treated here. An overview of our results is given at the end of the chapter.

Chapter 4 concerns the study of cones of games which are closed under the minimum operation. A well-known example of a minimum closed cone of games is the class of non-negative games with the superadditivity property. The theory that has been developed is applied to this class of games, resulting in a partial characterization of its extreme games.

An other approach of characterizing extreme superadditive games (a full characterization is still unknown) is by relaxing the superadditivity property. The last chapter is devoted to three classes of games which arise in this way. A result with respect to one of these classes shows that its extreme games can be of use in identifying extreme superadditive games. An other class is related to the games for which the core and the domination core are equal.

\section{Acknowledgements}

I wish to thank all persons who made it possible for me to accomplish this dissertation. First of all I like to thank Stef Tijs for introducing me in the fields of Operations Research and Game Theory. Most of the basic ideas which led to the results in this monograph arose during several stimulating discussions with Stef during the two and a half years I was a member of the $\Omega$-group in Nijmegen.

I want to express my gratitude to Koos Vrieze and Hans Peters for the encouragements, support and confidence they showed in the progress of this work.

Furthermore, I wish to thank all members of the department of mathematics in Maastricht for their support and pleasant and stimulating conversations.

Last but not least I like to thank Marlies Haenen for her IATEXtual support, Miriam Tillie who had the comprehensive task of correcting the English of this manuscript, and my wife Gerrie. Her support made this work really possible. 


\section{Notations and symbols}

Let $I R$ denote the set of scallars or reals. For a Euclidean vector space $I R^{P}$, with $P$ a finite index set, the inner product of two vectors $x, y \in I R^{P}$, $\sum_{i \in P} x_{i} y_{i}$, is denoted by $x \cdot y$. For a vector $x$ the norm $\|x\|$ equals $\sqrt{x \cdot x}$. We will sometimes represent an $x \in I R^{P}$ as a collection $\left(x_{i}\right)_{i \in P}$ of scalars.

For two vectors $x, y \in I R^{P}$ we denote $x_{i}<y_{i}$ for all $i \in P$ simply by $x<y$. The inequalities $x \leq y, x>y$, and $x \geq y$ are defined similarly.

For two subsets $X, Y$ of $I R^{P}$ the inclusion $X \subset Y$ is to be regarded as strict: $X \subseteq Y$ and $X \neq Y$. The algebraic sum $X+Y$ equals $\{x+y: x \in X$ and $y \in Y\}$, with the assumption that if one of the sets equals the empty set $\emptyset$ then the sum is the empty set. The set $\alpha X$, with scalar $\alpha \in I R$, denotes the set $\{\alpha x: x \in X\}$. For a scalar $\alpha$ the absolute value is denoted by $|\alpha|$. For a set $X$ the expression $|X|$ denotes the number of elements of $X$.

The symbol $\square$ denotes the end of the proof.

Let us finally list the symbols which will be defined at the moment they are used. In the listing the number denotes the page on which the corresponding symbol is defined.

$\begin{array}{llrl}1 & N & 4 & u^{S} \\ 2 & 2^{N} & 4 & \mathbf{1}^{S} \\ 2 & \langle N, v\rangle & 6 & \operatorname{Imp}(v) \\ 2 & \mathbf{G}^{N} & 6 & \operatorname{Core}(v) \\ 2 & I R^{2^{N} \backslash\{\emptyset\}} & 7 & \chi^{S} \\ 2 & \operatorname{car}^{Q} & 8 & \operatorname{DCore}(v) \\ 2 & v_{\mid S} & 9 & x^{\pi} \\ 3 & v_{0} & 10 & \mathrm{~W}(v) \\ 3 & v_{01} & 11 & b^{v} \\ 3 & v_{\text {one }} & 11 & \operatorname{MCore}(v)\end{array}$


xiv

$11 \gamma^{v}$

$13 \quad \mathrm{M}(v)$

$13 \quad a^{v}$

14 QCore $(v)$

$16 \phi(v)$

$17 \tau^{v}$

$18 \mathrm{MN}^{N}$

$18 \mathrm{SA}^{N}$

$18 \mathrm{CV}^{N}$

$18 \mathrm{~B}^{N}$

$18 \mathrm{~TB}^{N}$

$18 \mathrm{C}^{N}$

$18 \mathrm{~A}^{N}$

$18 \mathrm{~S}^{N}$

$18 \mathrm{Q}^{N}$

$18 \mathrm{MB}^{N}$

$18 \quad 1 \mathrm{C}^{N}$

$18 \quad \mathrm{~S}_{0}^{N}$

$18 \mathrm{~S}_{+}^{N}$

$19 c_{y}$

$19 h^{c}$

$19 w^{y}$

$19 v^{*}$

$19 g^{v}$

21 Cone $(X)$

$21 \operatorname{Conv}(X)$

$21 \quad \operatorname{Span}(X)$

$21 \quad H(y)$

$22 \quad \operatorname{Extr}(C)$

$22 \quad[a, b]$

$22 \quad \operatorname{mix}_{a}(b$,

$22 \operatorname{mix}_{J}(b)$
$24 \quad \operatorname{EDir}(C)$

$26 u^{x}$

$37 \quad \mathbf{D}^{N}$

$37 \quad \mathbf{H}^{N}$

$47 \quad \mathcal{N}(y)$

50 PMA

$50 \quad \mathbf{P M}^{N}$

$53 \quad w^{S}$

$53 \quad \mathrm{AC}^{N}$

$56 v^{c}$

$63 \quad v^{y}$

$64 \mathrm{MC}$

$68 U^{X}$

$70 \quad P$

$70 s$

$70 \quad t$

$70 \quad C(p, q)$

$71 \quad \nu(f)$

$71 \quad f(Q, P)$

$71 \quad V^{\mathbb{T}}$

$74 \quad W(p, q)$

$84 \hat{w}$

95 CLAN $^{N, C}$

$95 f_{C}$

$97 \quad \mathrm{SC}^{N}$

$104 v^{S}$

$117 \quad c_{S}$

$131 \quad \operatorname{Ess}\left(v, \mathbf{W}^{N}\right)$

$131 v_{\min }$

$141 \mathrm{~L}^{N}$

$153 \bar{v}$ 


\section{Chapter 1}

\section{Preliminaries}

\subsection{Introduction}

This monograph deals with the so-called transferable utility games, a subject of cooperative game theory. Here we will introduce and discuss the involved game theoretic notions and set a mathematical base for the chapters to come.

\section{The contents}

Section 1.2 begins with an introduction of the game theoretic model and some basic notions. Much attention is paid to the notion of the core. We will discuss three core catchers, which are sets containing the core as a subset; these are the Weber set, the marginal core and the core cover. Two single-valued solution schemes will be treated, the Shapley value and the $\tau$-value. Furthermore, games arising from situations in which costs are involved, rather than savings, will be discussed.

Finally, we will discuss some notions and preliminary results on cones and, in particular, on cones of games.

\subsection{Cooperative games}

\subsubsection{The game model}

The game model considered here consists of two components.

The player set

The first component is a finite and non-empty set of players, denoted by $N$. It is often convenient to number the players, i.e., to assume that $N$ is 
equal to $\{1,2, \ldots, n\}$, where $n$ denotes the number of players. The players are free to cooperate and a subset of players is thus called a coalition.

\section{The characteristic function}

The second component is the so-called characteristic function which is a real-valued function on the set $2^{N}$ of coalitions of $N$, assigning 0 to the empty set $\emptyset$. The ordered pair $\langle N, v\rangle$, with the player set $N$ and characteristic function $v: 2^{N} \longrightarrow I R$ is called a cooperative game or game for short. For a coalition $S \subseteq N$ the worth $v(S)$ is interpreted as the savings which can be obtained by $S$ in case the players in $S$ decide to cooperate.

Normally a game is identified with its characteristic function. In case this leads to confusion we will explicitly mention the player set. The set of games (with player set $N$ ) is denoted by $\mathbf{G}^{N}$. It is considered as the $\left(2^{|N|}-1\right)$-dimensional Euclidean vector space $I R^{2^{N} \backslash\{\emptyset\}}$, where its axes are indexed by the non-empty coalitions. Addition of games and multiplication by a real scalar are hereby defined.

\section{An example}

An example of a game is the so-called glove game, where the player set $N$ is the union of two finite sets $P$ and $Q$. Assume that a player in $P$ possesses a left hand glove and a player in $Q$ a right hand glove. Here, the worth of a coalition $S \subseteq N$ is determined by the number of pairs of gloves that can be formed by the members of $S$, and is given by the expression

$$
v(S)=\min (|S \cap P|,|S \cap Q|)
$$

Here $|S|$ denotes the number of players in coalition $S$.

The situation $N=P=Q$ gives rise to the so-called cardinality game $\operatorname{car}^{Q}$ (with respect to coalition $Q$ ) defined by $\operatorname{car}^{Q}(S)=|S \cap Q|$ for all $S \subseteq N$.

\section{Subgames}

The subgame of a game $v$ relative to a non-empty coalition $S$ is the game $\left\langle S, v_{\mid S}\right\rangle$ where $v_{\mid S}$ is defined to be the restriction of $v$ to the subsets of $S$. It is mathematically convenient to let a subgame of $v$ have the same player set as the game $v$. It is not confusing to define the game $v_{\mid S}$ alternatively as

$$
v_{\mid S}(T)=v(S \cap T) \text { for each coalition } T \subseteq N
$$


The 0,1-normalization

For a game $v$ the 0 -normalization $v_{0} \in \mathbf{G}^{N}$ of $v$ is defined by

$$
v_{0}(S)=v(S)-\sum_{j \in S} v(\{j\}) \text { for each } S \subseteq N .
$$

It arises from $v$ after the distribution of the worths which can be obtained by the single players without cooperation. Note that the worths of the oneperson coalitions are zero in $v_{0}$. A game $v$ for which $v(\{i\})=0$ for each player $i \in N$, yielding $v=v_{0}$, is thus called 0 -normalized. Two games $v$ and $w$ are said to be strategically equivalent if their 0-normalizations are equal up to a positive multiplication factor.

For a game $v$ with $v_{0}(N)>0$, i.e., $v(N)>\sum_{j \in N} v(\{j\})$, we call the game $v_{01}$, with

$$
v_{01}(S)=\frac{v_{0}(S)}{v_{0}(N)} \text { for each coalition } S,
$$

the 0,1 -normalization of $v$.

Simple games

A game $v$ is called non-negative if the worths of the coalitions are nonnegative. A game $v$ is called simple if the worths of the coalitions admit only two values, 0 and 1 , and the worth of the grand coalition $N$ equals 1 , i.e., $v(S) \in\{0,1\}$ for $\neq S \subset N$ and $v(N)=1$. The values 0 and 1 are usually associated with losing and winning (cf. Shapley [1956]). The all-winning game is denoted by vone, i.e.,

$$
v_{\text {one }}(S)=1 \text { for each non-empty coalition } S \text {. }
$$

In cases where it is not confusing we will denote the all-losing game simply by 0 . Notice that the all-losing game is not a simple game.

As an example consider the following voting situation: each player $i \in N$ has a voting weight $q_{i} \in I R_{+}$and there is a voting treshold $Q \in I R_{+}$with $Q \leq \sum_{j \in N} q_{j}$ (in many common voting situations the weights equal 1 and the treshold equals half of the number of voters/players, the so called majority rule); corresponding to this situation a simple game can be considered which assigns to each coalition $S$ the value 1 if the voting weights of the players in $S$ exceed the treshold, i.e., $\sum_{j \in S} q_{j} \geq Q$, and the value 0 otherwise. Coalitions, which are 1-valued, are thus called winning and 0-valued coalitions losing.

A player $i$ is a veto-player in a simple game if each coalition $S$ that does not contain $i$ has worth 0 . If a veto-player exists then the game is called vetocontrolled. For example, in the simple game arising from the above voting 
situation a player $i$ is a veto-player if $Q>\sum_{j \in N \backslash\{i\}} q_{j}$. Other examples of simple games with veto-players are the so-called unanimity games $u^{\mathcal{S}}, \emptyset \neq$ $S \subseteq N$, defined by

$$
u^{S}(T)=\left\{\begin{array}{ll}
1 & \text { if } T \supseteq S, \\
0 & \text { otherwise },
\end{array} \quad \text { for each } T \subseteq N\right.
$$

Observe that each member of $S$ is a veto-player in $u^{S}$. The unanimity games associated to the one-person coalitions are also called the dictator games. Notice that the cardinality game car ${ }^{\mathcal{S}}$ equals the sum of the dictator games corresponding to the players in $S$.

Other examples of $\{0,1\}$-valued games are the unity games $\mathbb{1}^{S}, S \subseteq N$, $S \neq \emptyset$, defined by

$$
\mathbb{I I}^{S}(T)=\left\{\begin{array}{ll}
1 & \text { if } S=T, \\
0 & \text { otherwise, }
\end{array} \text { for each } T \subseteq N .\right.
$$

Only $\mathbb{1}^{N}$ is a simple game.

Inequality conditions

The glove games and the games arising from voting situations are monotonic, i.e.,

$$
v(S) \leq v(T) \text { for all non-empty coalitions } S \subseteq T \text {. }
$$

It expresses the property that coalitions possess savings exceding those of the subcoalitions. If $v$ satisfies the condition

$$
v(S)+v(T) \leq v(S \cup T) \text { for all disjoint coalitions } S, T \subseteq N,
$$

then it is advantageous for already existing disjoint coalitions to join into larger coalitions. Games which fulfil (1.2) are called superadditive.

Games satisfying

$$
v(S)+v(T) \leq v(S \cup T)+v(S \cap T) \text { for all coalitions } S, T \subseteq N,
$$

are called convex. Of course, a convex game is superadditive. As we shall see later on (theorem 1.5) there is a strong incentive among the players in a convex game to form the grand coalition $N$. This may not be the case in a superadditive game as one can easily observe in the simple game $w$ with $w(S)=1$ only for coalitions $S$ with at least more than half of the 
players. This game corresponds to the majority rule voting situation and its superadditivity is easily checked.

\section{Balancedness}

Finally, we will mention here the so-called balancedness condition. Let $B$ be a set of non-empty coalitions. A collection $\left(\lambda_{S}\right)_{s \in B}$ of positive weights is called balanced if

$$
\sum_{S \in B, S \ni i} \lambda_{S}=1 \text { for each player } i \in N,
$$

and $B \subseteq 2^{N} \backslash\{\emptyset\}$ is called a balanced family of coalitions if a collection $\left(\lambda_{S}\right)_{S \in B}$ of positive weights exists for which (1.4) holds.

An interpretation of a collection $\left(\lambda_{S}\right)_{S \in B}$ is to consider a weight $\lambda_{S}$ as the (relative) length of time the players in the coalition $S \in B$ are in cooperation. The weights form a balanced collection, up to a scalar multiplication, if the total time a player is in cooperation is equal for each player. As an example consider the set $N$ to be $\{1,2,3\}$ and $B$ is the set of the two-person coalitions of $N$. If the corresponding weights all equal $\frac{1}{2}$ then these weights form a balanced collection since each of the three players is contained in exactly two of the coalitions in $B$.

A game $v$ is called balanced if it fulfils

$$
\sum_{S \in B} \lambda_{S} v(S) \leq v(N) \text { for each balanced collection }\left(\lambda_{S}\right)_{S \in B}
$$

The game $v$ is said to be totally balanced if each subgame is balanced.

\subsubsection{The core and the domination core}

One of the goals of cooperative game theory is to obtain reasonable rulles which reflect the strength of a player in a game. In this context the following notions appear.

\section{Allocations}

An element $x=\left(x_{i}\right)_{i \in N} \in I R^{N}$ is called an allocation. It can be interpreted to represent a distribution of strength or savings among the players. In a game $v$ an allocation $x$ is called efficient if $x$ denotes a distribution of the value of $N$ among the players, i.e.,

$$
\sum_{j \in N} x_{j}=v(N)
$$


A (set-valued) function on a subset of the game space is called a solution scheme if it assigns to a game one (a set of) efficient allocation(s) of that game.

An allocation $x$ is said to be individually rational if for each player $i$ his distribution $x_{i}$ exceeds the worth he can get by operating on his own, i.e.,

$$
x_{i} \geq v(\{i\}) \quad \text { for each player } i \in N .
$$

Let $\operatorname{Imp}(v)$ denote the set of allocations which are both efficient and individually rational in the game $v$, i.e.,

$$
\operatorname{Imp}(v)=\left\{x \in I R^{N}: \sum_{j \in N} x_{j}=v(N) \text { and } x \geq(v(\{i\}))_{i \in N}\right\} .
$$

This set is called the imputation set of the game $v$.

The imputation set, considered as a set-valued function may serve as an example of a solution scheme. The best-known and most widely applied solution scheme, however, is the core.

\section{The core}

If for an efficient allocation $x$ we have $\sum_{j \in S} x_{j} \geq v(S)$ for each coalition $S$ then $x$ is called a core allocation of the game $v$. The set of all core allocations is denoted by Core $(v)$,

$$
\operatorname{Core}(v)=\left\{x \in I R^{N}: \sum_{j \in N} x_{j}=v(N) \text { and } \sum_{j \in S} x_{j} \geq v(S) \text { for each } S \subset N\right\}
$$

In games with a non-empty core there is an incentive among the players to cooperate and form the grand coalition $N$. Evidently, by choosing a core allocation as a distribution of the savings $v(N)$ the formation of smaller coalitions will be discouraged since the worth of each of these coalitions does not exceed the amount the players receive from the chosen allocation.

For example, in an additive game $v$, i.e.,

$$
v(S)=\sum_{j \in S} v(\{j\}) \text { for each non-empty coalition } S,
$$

the only reasonable division of the total savings $v(N)$ among the players is the allocation of the amount $v(\{i\})$ to each player $i$. This distribution represents a core allocation of $v$; in fact it is the only core allocation.

Convexity of a game implies the non-emptiness of its core as we will discuss in the next subsection. 
Using a core allocation $x$ of a game $v$, the balancedness of $v$ follows from

$$
\sum_{S \in B} \lambda_{S} v(S) \leq \sum_{S \in B} \lambda_{S}\left(\sum_{j \in S} x_{j}\right)=\sum_{j \in N} x_{j}\left(\sum_{S \in B, j \in S} \lambda_{S}\right)=\sum_{j \in N} x_{j}=v(N)
$$

for each balanced collection $\left(\lambda_{S}\right)_{S \in B}$. Also the reverse holds, i.e., a balanced game has a non-empty core (Bondareva [1963], Shapley [1967]). We will discuss this equivalence in subsection 2.2 .2 .

The veto-controlled simple games

It is a well-known result that a veto-controlled simple game $v$ has a nonempty core. In fact we have

Theorem 1.1 For a simple game $v$ the core equals the convex hull of the allocations $\chi^{\{i\}}$, with $i \in N$ a veto-player of $v$.

Here $\chi^{\mathcal{S}}$ denotes the allocation which assigns 1 to each player in the coalition $S$ and 0 to players outside $S$.

Proof: Observe that for a veto-player $i$ the allocation $\chi^{\{i\}}$ is an element of the core of $v$. The convexity property of the core is obvious, yielding $\operatorname{Conv}\left(\left\{\chi^{\{i\}}\right.\right.$ : i a veto-player of $\left.\left.v\right\}\right) \subseteq \operatorname{Core}(v)$. Of course, equality holds in case Core $(v)$ is empty. Thus, suppose $\operatorname{Core}(v)$ is non-empty, say $x \in \operatorname{Core}(v)$. Now $x$ consists of non-negative coefficients summing up to $v(N)=1$. Let $i \in N$ be a player for which $x_{i}>0$. For each coalition $S$ not containing $i$ we have

$$
v(S) \leq \sum_{j \in S} x_{j} \leq \sum_{j \in N \backslash\{i\}} x_{j}=1-x_{i}<1,
$$

implying $v(S)=0$. Therefore, $i$ must be a veto-player. Hence,

$$
x=\sum\left\{x_{i} \chi^{\{i\}}: i \text { a veto-player of } v\right\}
$$

implying Core $(v) \subseteq \operatorname{Conv}\left(\left\{\chi^{\{i\}}:\right.\right.$ i a veto-player of $\left.\left.v\right\}\right)$. This completes the proof.

Of course, it follows that the veto-controlled simple games are precisely the simple games with non-empty core:

Corollary 1.2 For a simple game the core is non-empty if and only if there is a veto-player.

\section{The domination core}

The concept of the core is introduced in Gillies [1953]. Gillies' definition of the core, however, is slightly different from the one above; it consists of 
the so-called undominated allocations. In a game $v$ an allocation $x \in \operatorname{Imp}(v)$ is said to be dominated by $y \in \operatorname{Imp}(v)$ if there is a coalition $S$ for which

- $\sum_{j \in S} y_{j} \leq v(S)$, and

- $y_{i}>x_{i}$ for each player $i \in S$.

Thus, the allocation $x$ can be improved for all players in $S$ simply by forming the coalition $S$ and distribute at most the worth $v(S)$ in accordance with the pay-offs $y_{i}, i \in S$. The set of all allocations in $\operatorname{Imp}(v)$ which are not dominated is called the domination core and is denoted by DCore $(v)$.

Observe that a core allocation cannot be dominated. Therefore, the core is always a subset of the domination core. It is well-known that the core and domination core coincide on the class of superadditive games. It is less well-known that the equality $\operatorname{Core}(v)=\operatorname{DCore}(v)$ also holds for games for which the weaker condition

$$
v(N) \geq v(S)+\sum_{j \notin S} v(\{j\}) \quad \text { for each coalition } S
$$

holds; this is equivalent with the property that the worth of the grand coalition in the corresponding 0-normalized game is largest among the worths of all non-empty coalitions. In a certain way also the reverse is true as the following theorem shows.

Theorem 1.3 The core of a game $v$ equals the domination core DCore(v) if and only if DCore(v) is empty or $v$ fulfils (1.6).

Proof: Since the inclusion Core $(v) \subseteq$ DCore $(v)$ always holds the theorem only has to be proved for the case the domination core is non-empty. ('only if'-part) Suppose Core $(v)=\operatorname{DCore}(v)$. For an arbitrary $x \in$ Core $(v)$ and $S \subseteq N$ we have

$$
v(N)=\sum_{j \in S} x_{j}+\sum_{j \notin S} x_{j} \geq v(S)+\sum_{j \notin S} v(\{j\}),
$$

implying (1.6).

('if'-part) Suppose Core $(v) \neq$ DCore $(v)$, say $x \in \operatorname{DCore}(v) \backslash$ Core $(v)$. Let coalition $S$ be such that $\sum_{j \in S} x_{j}<v(S)$. Note that from $x \in \operatorname{Imp}(v)$ it follows that $S$ contains more than 1 player and is unequal to $N$. Consider the allocation $y$ with

$$
y_{i}=\left\{\begin{array}{cc}
x_{i}+|S|^{-1}\left(v(S)-\sum_{j \in S} x_{j}\right) & \text { if } i \in S, \\
v(\{i\})+|N \backslash S|^{-1}\left(v(N)-v(S)-\sum_{j \notin S} v(\{j\})\right) & \text { if } i \notin S .
\end{array}\right.
$$


Then $\sum_{j \in S} y_{j}=v(S)$, and $y_{i}>x_{i}$ for all $i \in S$. The allocation $y$ cannot dominate $x$ and, therefore, it is not an element of $\operatorname{Imp}(v) . y$ is clearly efficient and $y_{i}>x_{i} \geq v(\{i\})$ for all $i \in S$. Therefore, there is a player $i \notin S$ with $y_{i}<v(\{i\})$, implying $v(N)-v(S)-\sum_{j \notin S} v(\{j\})<0$. Thus, (1.6) is not fulfilled by $v$. The theorem now follows.

From theorem 1.3 it follows that the core of a game must be empty whenever it does not coincide with the domination core (cf. Van den Nouweland, Potters, Tijs and Zarzuelo [1990]).

\subsubsection{Core catchers}

The problem of constructing a core allocation for a game can be tackled by using linear programming techniques. There are no game theoretic methods available in the general case. Many types of games, however, have been studied for which core allocations can be computed in a predescribed way or for which the core is a well described set in the allocation space (see for example theorem 1.1 regarding the characterization of the core for the simple games).

Partly to avoid the computational complexity of the core other sets of efficient allocations have been introduced with nice computational properties and containing the core as a subset. Here we will discuss three of these socalled core catchers. For two of these allocation sets a characterization is given of those games for which the core coincide with the considered core catcher.

\section{The Weber set}

Consider the situation that the players in a game $v$ decide to distribute the worth $v(N)$ among themselves in the following way. First an arbitrary sequence of the players, say $\pi(1), \pi(2), \ldots, \pi(n)$, is chosen, with $n=|N|$. Now if the grand coalition is formed by subsequently adding a player in the order given by $\pi$ then the contribution of player $\pi(j)$ to the worth of $N$ equals

$$
x_{\pi(j)}^{\pi}=v(\{\pi(1), \pi(2), \ldots, \pi(j)\})-v(\{\pi(1), \pi(2), \ldots, \pi(j-1)\}) .
$$

The allocation $x^{\pi}$ is called a marginal worth allocation (with respect to the sequence $\pi$ ). The sum of the coordinates of $x^{\pi}$ equals $v(N)$ according to

$$
\sum_{j=1}^{s} x_{\pi(j)}^{\pi}=v(\{\pi(1), \pi(2), \ldots, \pi(s)\}) \quad \text { for each } s \text { with } 1 \leq s \leq n
$$


thus the marginal worth allocations are efficient. Notice that there are $n$ ! different sequences. However, some of the corresponding marginal worth allocations may coincide.

Weber [1988] showed that the convex hull of the marginal worth allocations of an arbitrary game $v$, denoted by $W(v)$ and referred to as the Weber set, is a core catcher, i.e.,

Theorem 1.4 (Weber) The core of a game is included in the Weber set.

The standard proof of this theorem uses an induction argument on the number of players in $N$. We will give here a short proof based on a well-known result in convex analysis (usually we shall not include a (full) proof of referenced results).

Proof: Let us suppose that theorem 1.4 is not true. Then there is a game $v$ with a core allocation, say $x$, which is not contained in the Weber set. The well-known Separation Theorem (cf. Rockafellar [1970]), together with the convexity and closedness of the Weber set implies that there exists a vector $y \in I R^{N}$ and a scalar $\alpha$ such that the inner product $z \cdot y$ exceeds $\alpha$ for each $z \in \mathrm{W}(v)$ and $x \cdot y<\alpha$. Let $\pi$ be a sequence of players such that $y_{\pi(1)} \geq y_{\pi(2)} \geq \ldots \geq y_{\pi(n)}$. Let us consider the inner product of the marginal worth allocation $x^{\pi}$ and $y$ :

$$
\begin{aligned}
\alpha & \leq x^{\pi} \cdot y \\
& =\sum_{i=1}^{n} y_{\pi(i)}(v(\{\pi(1), \pi(2), \ldots, \pi(i)\})-v(\{\pi(1), \pi(2), \ldots, \pi(i-1)\})) \\
& =y_{\pi(n)} v(N)+\sum_{i=1}^{n-1}\left(y_{\pi(i)}-y_{\pi(i+1)}\right) v(\{\pi(1), \pi(2), \ldots, \pi(i)\}) .
\end{aligned}
$$

Using $x \in \operatorname{Core}(v)$ and $y_{\pi(i)}-y_{\pi(i+1)} \geq 0$ for all $1 \leq i \leq n-1$, we have

$$
\begin{aligned}
\alpha & \leq y_{\pi(n)} \sum_{j=1}^{n} x_{\pi(j)}+\sum_{i=1}^{n-1}\left(y_{\pi(i)}-y_{\pi(i+1)}\right) \sum_{j=1}^{i} x_{\pi(j)} \\
& =\sum_{i=1}^{n} y_{\pi(i)} \sum_{j=1}^{i} x_{\pi(j)}-\sum_{i=2}^{n} y_{\pi(i)} \sum_{j=1}^{i-1} x_{\pi(j)} \\
& =\sum_{j=1}^{n} y_{\pi(j)} x_{\pi(j)}
\end{aligned}
$$

and this is contradictory to our assumption that the inner product of $y$ and $x$ is strictly smaller than $\alpha$. 
We conclude that the theorem holds for all games.

The core and the Weber set coincide precisely for the convex games:

Theorem 1.5 For a game $v$ we have equality Core $(v)=W(v)$ if and only if $v$ is convex.

The 'if' part of the proof is a well-known result of Shapley [1971] and the 'only-if' part has been shown in Ichiishi [1981].

Observe that the set $W(v)$ is always non-empty. Therefore, the core of a convex game is non-empty.

Other core catchers with nice computational properties have been introduced in Tijs and Lipperts [1982]. In order to introduce these allocation sets we will first associate two other (not necessarily efficient) allocations to a game $v$, the marginal contribution allocation $b^{v}$ and the maximal remainder allocation $a^{v}$.

The marginal contribution allocation

The marginal contribution allocation $b^{v} \in I R^{N}$ is defined as

$$
b_{i}^{v}=v(N)-v(N \backslash\{i\}), i \in N .
$$

Assuming that the players in a game $v$ first try to form the grand coalition, we assert that a player $i$ cannot demand a greater portion of the worth $v(N)$ than the amount $b_{i}^{v}$, since he would otherwise force the other players to divide an amount less than $v(N)-b_{i}^{v}=v(N \backslash\{i\})$ among themselves. Thus, only those efficient allocations which are majorized by the marginal contribution allocation are considered. The set of these allocations is called the marginal core of $v$ and is denoted by MCore $(v)$ :

$$
\operatorname{MCore}(v)=\left\{x \in I R^{N}: x \leq b^{v} \text { and } \sum_{j \in N} x_{j}=v(N)\right\} \text {. }
$$

It is clear that MCore $(v) \neq 0$ if and only if

$$
v(N) \leq \sum_{j \in N} b_{j}^{v}
$$

Notice that $\operatorname{MCore}(v)$ is the convex hull of the efficient allocations $f^{j}=b^{v}-$

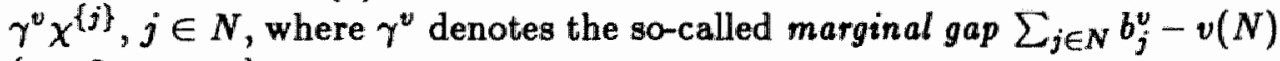
(see figure 1.1). 


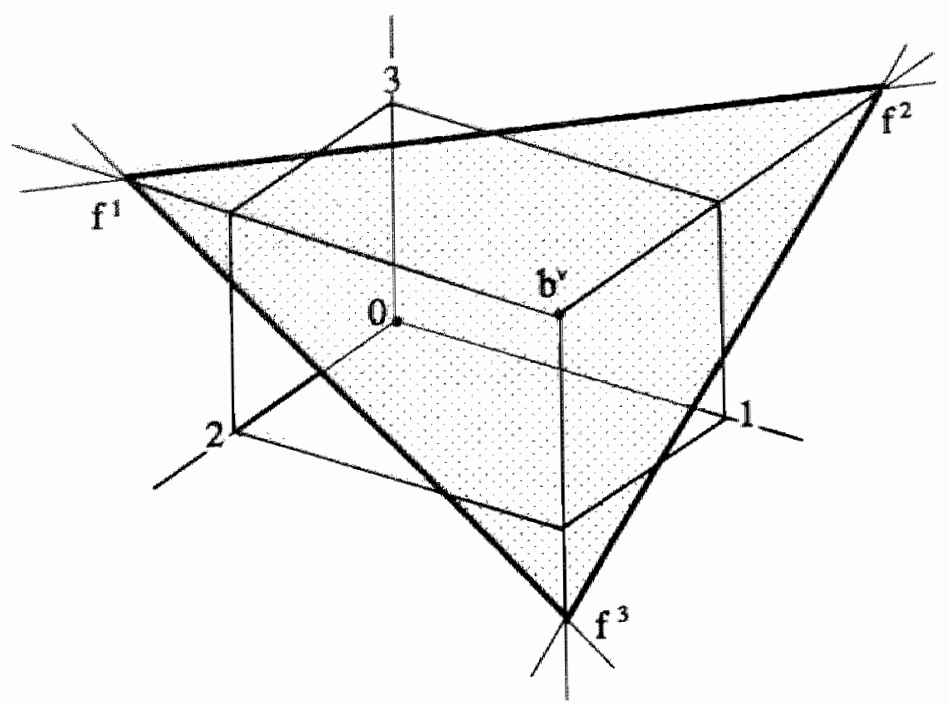

Figure 1.1: The allocation set MCore $(v)$

For a core allocation $x$ of a game $v$ we have

$$
x_{i}=v(N)-\sum_{j \in N \backslash\{i\}} x_{j} \leq v(N)-v(N \backslash\{i\})=b_{i}^{v} \quad \text { for each player } i
$$

yielding

Theorem 1.6 The core of a game is contained in the marginal core.

The marginal core is introduced in Tijs and Lipperts [1982]. In Driessen and Tijs [1983] games are considered for which the core equals the marginal core. These games are characterized by the following

Theorem 1.7 (Driessen, Tijs) Core $(v)=M \operatorname{Core}(v)$ if and only if MCore $(v)$ is empty or MCore $(v) \neq 0$, and the game $v$ fulfils

$$
v(S) \leq v(N)-\sum_{j \in N \backslash S} b_{j}^{v} \quad \text { for each non-empty coalition } S \subseteq N .
$$

Games fulfilling (1.9) are called maximally blocked. The maximally blocked games for which also the marginal core is non-empty are called 1-convex. From theorem 1.7 it thus follows that the games with a non-empty core equal to the marginal core are precisely the 1-convex games. For an extensive discussion on 1-convex games we refer the reader to Driessen [1985] and Driessen [1988]. 


\section{The Milnor set}

The marginal core of a game $v$ is a subset of the allocation set

$$
\begin{aligned}
\mathrm{M}(v)=\left\{x \in I R^{N}:\right. & \sum_{j \in N} x_{i}=v(N) \text { and } x_{i} \leq v(S \cup\{i\})-v(S) \\
& \text { for at least one } S \subseteq N \backslash\{i\}, i \in N\} .
\end{aligned}
$$

$M(v)$ contains the efficient allocations of a game $v$ for which the pay-off to a player $i$ is majorized by his marginal contribution to one of the coalitions $S \subseteq N \backslash\{i\}$. This set is first considered in Milnor[1952] (see also Luce and Raiffa [1957], chapter 11).

The semibalanced games

In distributing the worth $v(N)$ among the players in $N$ we argued that only allocations in MCore $(v)$ are to be considered. Now if a non-empty coalition $S \neq N$ exists with $v(S)>\sum_{j \in S} b_{j}^{v}$ then the players in $S$ will not be satisfied by any (efficient) allocation which is majorized by $b^{v}$. It seems, therefore, reasonable to examine the formation of the grand coalition only in a game $v$ that fulfils

$$
v(S) \leq \sum_{j \in S} b_{j}^{\mathrm{v}} \quad \text { for each non-empty coalition } S .
$$

Games with this property are called semibalanced and were first discussed in Tijs and Lipperts [1982].

A 1-convex game $v$ is semibalanced. This follows from the fact that the non-emptiness of its marginal core implies the inequality $v(N) \leq \sum_{j \in N} b_{j}^{v}$. Now using this inequality in the conditions (1.9) we obtain the semibalancedness conditions (1.10).

\section{The maximal remainder allocation}

Now let $v$ be a semibalanced game. Suppose player $i \in N$ is interested in forming a coalition $S$ and he is willing to pay the members $j \in S \backslash\{i\}$ the payoff $b_{j}^{v}$. For $i$ the amount of $v(S)-\sum_{j \in S \backslash\{i\}} b_{j}^{u}$ remains. Of course, player $i$ is interested in forming a coalition for which this is maximal. Let us denote this amount by $a_{i}^{v}$, the maximal remainder for player $i$ (cf. Tijs [1981]). Hence,

$$
\begin{aligned}
a_{i}^{v} & =\max _{S \ni i}\left(v(S)-\sum_{j \in S \backslash\{i\}} b_{j}^{v}\right) \\
& =b_{i}^{v}+\max _{S \ni i}\left(v(S)-\sum_{j \in S} b_{j}^{v}\right), \quad i \in N .
\end{aligned}
$$






Figure 1.2: The core cover

Note that

$$
b_{i}^{v}-a_{i}^{\nu}=\min _{S \ni i}\left(\sum_{j \in S} b_{j}^{\nu}-v(S)\right), \quad i \in N .
$$

Thus $b^{v} \geq a^{v}$ is equivalent to $(1.10)$, i.e.,

Lemma 1.8 A game $v$ is semibalanced if and only if $b^{v} \geq a^{*}$.

The maximal remainder allocation may serve as a lower bound for the claims of the players, and we call the set of efficient allocations majorizing $a^{v}$ and majorized by $b^{v}$ the core cover:

$$
\mathrm{QCore}(v)=\left\{x \in I R^{N}: a^{v} \leq x \leq b^{v} \text { and } \sum_{j \in N} x_{j}=v(N)\right\}
$$

This notion is introduced in Tijs and Lipperts [1982] as a core relaxation: Theorem 1.9 The core of a game is a subset of the core cover.

Its proof is simply based on the observations that a core allocation $x$ of $v$ is majorized by the marginal contribution allocation, and

$$
x_{i}=\sum_{j \in S} x_{j}-\sum_{j \in S \backslash\{i\}} x_{j} \geq v(S)-\sum_{j \in S \backslash\{i\}} x_{j} \geq v(S)-\sum_{j \in S \backslash\{i\}} b_{j}^{v}
$$

for each coalition $S$ containing player $i$; therefore, $x$ majorizes the maximal remainder allocation. 
Games for which the core and the core cover coincide are called ideally covered. There is no nice characterization available of ideally covered games using inequality conditions as done in the theorems 1.5 and 1.7 , due to the fact that the ideally covered games do not form a convex cone in the game space. See also subsection 3.5.2.

Observe that the core cover is a subset of the marginal core.

The quasi-balanced games

Games with a non-empty core cover are called quasi-balanced. According to lemma 1.8 quasi-balanced games are semibalanced. Furthermore, observe that semibalanced games $v$ for which also

$$
\sum_{j \in N} a_{j}^{v} \leq v(N)
$$

holds are quasi-balanced.

The notions semibalancedness and quasi-balancedness suggest a relationship with balancedness which is indeed the case (cf. Tijs [1981]). Each of the semibalancedness conditions $\sum_{j \in S} b_{j}^{v} \geq v(S)$, can be rephrased as

$$
v(S)+\sum_{j \in S} v(N \backslash\{j\}) \leq|S| v(N),
$$

and this corresponds to one of the balancedness conditions in (1.5), with balanced family $B=\{S\} \cup\{N \backslash\{i\}: i \in S\}$ and weights $|S|^{-1}$ for each of the coalitions in $B$.

Now consider (1.12). Using the definition of $a_{j}^{v}, j \in N$, we must have

$$
\sum_{i \in N} v\left(S^{i}\right)+\sum_{j \in N} \sum_{j \in S^{i} \backslash\{i\}} v(N \backslash\{j\}) \leq\left(1+\sum_{i \in N}\left(\left|S^{i}\right|-1\right)\right) v(N)
$$

for each collection $\left(S^{i}\right)_{i \in N}$ of coalitions satisfying $i \in S^{i}$. Consider the set $B=\left\{S^{i}: i \in N\right\} \cup\{N \backslash\{i\}: i \in N\}$ of coalitions which are involved in the above inequality. Then $B$ is a balanced family. In order to show this let us formulate a collection of weights $\left(\lambda_{S}\right)_{S \in B}$ for which (1.4) holds. Let $s$ denote the amount $1+\sum_{i \in N}\left(\left|S^{i}\right|-1\right)$. For a coalition $S \in B$ let $s_{S}$ denote the number of coalitions in the collection $\left(S^{i}\right)_{i \in N}$ which equals $S$. Furthermore, for a player $i$ let $t_{i}$ denote the number of coalitions in $\left(S^{i}\right)_{i \in N}$ containing player $i$; now consider $\lambda_{S}=s_{S} / s$ if $S \in B, S \neq N \backslash\{i\}$ for all players $i$, and $\lambda_{S}=\left(s_{S}+t_{i}\right) / s$ if $S=N \backslash\{i\}, i \in N$. Using the property $i \in S^{i}, i \in N$, it is a straightforward exercise to check that the weights $\left(\lambda_{S}\right)_{S \in B}$ indeed 
fulfil the balancedness requirements (1.4), and that the inequality (1.13) is equivalent to $\sum_{S \in B} \lambda_{S} v(S) \leq v(N)$.

We conclude that the definitions of semibalancedness and quasi-balancedness devolves to some of the balancedness inequalities.

\subsubsection{Single-valued solution schemes}

Among the considered efficient allocation sets, the core and domination core, regarded as set-valued solution schemes, have been discussed extensively in literature. Other well-known set-valued solution schemes are the Bargaining Set (Aumann and Maschler [1964]), the Kernel (Davis and Maschler [1965]) and the Prekernel (Maschler, Peleg and Shapley [1972]).

Here we will discuss two single-valued solution schemes. The nucleolus (Schmeidler [1969]) and the prenucleolus (Sobolev [1975]), although generally accepted, are left out of consideration.

\section{The Shapley value}

The first and best-known single-valued solution scheme is the so-called Shapley value, which is introduced in Shapley [1953]. For a game $v$ we define its Shapley value $\phi(v)$ as the average of the $|N|$ ! marginal worth allocations of the game $v$. It is, therefore, an efficient allocation which is contained in the Weber set. It is, however, not the 'centre of gravity' of W(v) since in general some of the marginal worth allocations may coincide. Notice that the Shapley value of a convex game is contained in the core since it is a convex combination of the marginal worth allocations which are core allocations of a convex game.

The Shapley value has been characterized in many different ways. The following description is used most frequently.

$$
\phi(v)_{i}=\sum_{S \subseteq N \backslash\{i\}} \frac{|S| !(n-|S|-1) !}{n !}(v(S \cup\{i\})-v(S)), \quad i \in N,
$$

with $n=|N|$. It easily follows that the Shapley value is a linear function on the game space. It is well-known (see also subsection 3.6.2) that the unanimity games form a base of $\mathbf{G}^{N}$ and, thus, each game can be written as a linear combination of the unanimity games. The linearity of the Shapley value now implies that it is fully specified by its values $\phi\left(u^{S}\right)$ on each unanimity game $u^{S}, \neq S \subseteq N$. In order to compute $\phi\left(u^{S}\right)$ observe that every marginal worth allocation of $u^{S}$ equals $\chi^{\{i\}}$ for a player $i$ in $S$, and among the marginal worth allocations the allocations $\chi^{\{i\}}, i \in S$, occur equally often. These observations, together with the efficiency property of the Shapley 
value, imply

$$
\phi\left(u^{s}\right)=\frac{1}{|S|} x^{s}
$$

Intuitively, this allocation corresponds to the only reasonable distribution of the worth 1 of the grand coalition in $u^{s}$.

Additional information on the Shapley value can be obtained from any introductory book on Game Theory.

The $\tau$-value

The other solution scheme discussed here is the so-called $r$-value. It is introduced in Tijs [1981], and it is defined for the quasi-balanced games. For these games the core cover of efficient allocations, positioned between the maximal remainder allocation and the marginal contribution allocation, is non-empty, and the $\tau$-value $\tau^{v}$, of a quasi-balanced game $v$ is defined as the unique efficient allocation on the line segment with end points $a^{v}$ and $b^{v}$. It is, therefore, contained in the core cover QCore(v).

In the computation of the $\tau$-value of a game the main computational effort is determined by the calculation of the maximal remainder allocation. As an example let us consider the unanimity games again. The marginal contribution allocation of a unanimity game $u^{S}$ equals $\chi^{S}$ and, thus,

$$
\begin{array}{rlrl}
a_{i}^{u^{S}} & =\chi_{i}^{S}+\max _{T \ni i}\left(u^{S}(T)-\sum_{j \in T} \chi_{j}^{S}\right) & \\
& =0+0=0 & & \text { if } i \notin S, \\
& =1+u^{S}(\{i\})-1=u^{S}(\{i\}) & \text { if } i \in S,
\end{array}
$$

i.e., $a^{u^{S}}$ equals the zero allocation if $S$ consists of at least two players and in the case $S=\{i\}$ we have $a^{u^{(i)}}=\chi^{\{i\}}$. In both cases the $T$-value of a unanimity game $u^{S}$ equals its Shapley value $|S|^{-1} \chi^{S}$.

The game $-1^{S}$, with $\emptyset \neq S \subset N$, has a non-empty core, consisting of only one element, the zero allocation. Since the core is is a subset of the core cover these games must therefore be quasi-balanced and one can show easily that their maximal remainder allocation also equals the zero allocation. We conclude that $\tau^{-\mathbf{1}^{s}}=a^{-\mathbf{1}^{s}}=0$.

For additional information on the $\tau$-value the reader is referred to Driessen [1988], Driessen and Tijs [1985] and Tijs [1981]. For a computational comparison of the Shapley value and the $\tau$-value we refer to Dragan, Potters and Tijs [1989]. 


\subsubsection{Summary of the introduced classes of games}

In the table below the introduced types of games are summarized and a notation of the corresponding classes of games is given, which is used throughout the monograph. Also some of the presented results are listed.

In the table $S$ and $T$ are arbitrary non-empty coalitions.

\begin{tabular}{|c|c|c|}
\hline game property & class & description \\
\hline $\begin{array}{l}\text { monotonic } \\
\text { superadditive } \\
\text { convex } \\
\quad \text { eq. with } \\
\text { balanced } \\
\text { tot. balanced } \\
\text { non-empty core } \\
\text { additive } \\
\text { semibalanced } \\
\text { quasi-balanced } \\
\quad \text { eq. with } \\
\text { max. blocked } \\
\text { 1-convex } \\
\quad \text { eq. with }\end{array}$ & $\begin{array}{l}\mathbf{M} \mathbf{N}^{N} \\
\mathbf{S A}^{N} \\
\mathbf{C V}^{N} \\
\mathbf{B}^{N} \\
\mathbf{T B}^{N} \\
\mathbf{C}^{N} \\
\mathbf{A}^{N} \\
\mathbf{S}^{N} \\
\mathbf{Q}^{N} \\
\mathbf{M B}^{N} \\
\mathbf{1 C}^{N}\end{array}$ & $\begin{array}{l}v(S) \leq v(T) \quad \text { if } S \subseteq T \\
v(S)+v(T) \leq v(S \cup T) \quad \text { if } S \cap T=\emptyset \\
v(S)+v(T) \leq v(S \cup T)+v(S \cap T) \\
\text { Core }(v)=W(v) \\
\text { see }(1.5) \\
\text { see definition following (1.5) } \\
\text { Core }(v) \neq \emptyset \\
v(S)=\sum_{j \in S} v(\{j\}) \\
v(S) \leq \sum_{j \in S} b_{j}^{v} \\
Q \operatorname{Core}(v) \neq \emptyset \\
\sum_{j \in N} a_{j}^{v} \leq v(N) \text { and } v(S) \leq \sum_{j \in S} b_{j}^{v} \\
v(S) \leq v(N)-\sum_{j \in N \backslash S} b_{j}^{v} \\
v(N) \leq \sum_{j \in N} b_{j}^{v}, v(S) \leq v(N)-\sum_{j \in N \backslash S} b_{j}^{v} \\
\operatorname{Core}(v)=\operatorname{MCore}(v) \neq \emptyset\end{array}$ \\
\hline
\end{tabular}

The restriction of a class of games, e.g. $\mathbf{S}^{N}$, to the non-negative games, the 0 -normalized games and the 0,1-normalized games is denoted by $\mathbf{S}_{+}^{N}, \mathbf{S}_{0}^{N}$ and $\mathbf{S}_{01}^{N}$ respectively.

\subsubsection{Games arising from cost situations}

In the foregoing we considered the worths of the coalitions in a game to be savings or profits. Many economic situations, however, give rise to assignments of costs to the involved players and coalitions. For example, Littlechild and Owen [1974] and Littlechild [1973] studied the problem of setting airport landing charges for different types of aircraft. The game theoretic approach to the airport cost allocation problem is based on an appropriately defined function, the so-called airport cost function $c_{y}: 2^{N} \rightarrow I R$. Here $N$ is the set of planes which are to land at the airport and $y=\left(y_{i}\right)_{i \in N}$ denotes the costs for each plane $i \in N$ to construct a runway of appropiate length. Then 
costs for a coalition $S$ is determined by that plane in $S$ with the longest runway, i.e.,

$$
c_{y}(S)=\max _{i \in S} y_{i *}
$$

We allow the worths of the coalitions in a game to represent costs, rather than savings; in cases where ambiguity may occur we will denote games as cost games or savings games.

\section{The savings function}

With respect to a cost game $c$ we associate the savings game $h^{c}$ defined by

$$
h^{\mathfrak{c}}(S)=\sum_{j \in S} c(\{j\})-c(S) \text { for each non-empty coalition } S .
$$

This transformation function on the game space is referred to as the savings function.

The associated savings game of the airport cost function $c_{y}$ is referred to as the airport cost savings game $w^{\nu}$ (with respect to the cost allocation $\left.y \in I R^{N}\right)$,

$$
w^{y}(S)=\sum_{j \in S} y_{j}-\max _{i \in S} y_{i} \quad \text { for each non-empty coalition } S .
$$

This game actually has a non-empty core in case the cost vector $y$ is nonnegative: one easily checks that the efficient allocation $y-y_{i} \chi^{\{i\}}$, with player $i$ such that $y_{i}=\max _{j \in N} y_{j}$, is a core allocation.

\section{The gap function}

There are various possibilities to associate a cost game to a savings game $v$. One is the so-called dual game $v^{*}$ defined by

$$
v^{*}(S)=v(N)-v(N \backslash S) \text { for each non-empty coalition } S .
$$

The one which we will consider here into more detail is introduced in Driessen [1985]. To a game $v$ we associate the cost game $g^{v}$ defined by

$$
g^{v}(S)=\sum_{j \in S} b_{j}^{v}-v(S) \text { for each non-empty coalition } S \text {. }
$$

For each coalition $S$ the gap $\sum_{j \in S} b_{j}^{v}-v(S)$ represents the costs which arise after the distribution of the marginal contributions among the players in $S$. Observe that the marginal contribution allocation of the cost game $g^{*}$ 
equals the zero allocation. Cost games with this property correspond to cost allocation problems in which dismission of one person from the grand coalition does not influence the total costs which have to be made to meet the goals of the grand coalition. For example, the airport cost function fulfils this property whenever there are at least two planes with equal longest runways.

The above transformation function $g$ of a game into a cost game is also called the gap function. One easily proves that the gap function and the savings function are linear. In fact, they are injective on the class of 0 normalized games and on the class of cost games with zero marginal contribution allocation respectively. Actually, we have

Lemma 1.10 The gap function g restricted to $\mathbf{G}_{0}^{N}$ and the savings function $h$ restricted to $\left\{c \in \mathbf{G}^{N}: b^{c}=0\right\}$ are inverses of each other.

Proof: For each 0-normalized game $v$ we have, with coalition $S$ arbitrary,

$$
\begin{aligned}
h^{g^{*}}(S) & =\sum_{j \in S} g^{v}(\{j\})-g^{v}(S) \\
& =\sum_{j \in S}\left(b_{j}^{v}-v(\{j\})\right)-\left(\sum_{j \in S} b_{j}^{v}-v(S)\right) \\
& =v(S)-\sum_{j \in S} v(\{j\})=v(S) .
\end{aligned}
$$

Similarly, $g^{h^{c}}=c$ for each $c \in \mathbf{G}^{N}, b^{c}=0$.

\subsection{Polyhedral cones}

Let us first review some notions and results related to cones.

\subsubsection{Cones}

In a vector space $I R^{m}$ a set $C$ is called a (convex) cone if it fulfils

$$
\alpha x+\beta y \in C \text { for all } x, y \in C \text { and } \alpha, \beta \in I R_{+} .
$$

To a non-empty set $X \subseteq I R^{m}$ we associate the following cone

$$
\left\{\sum_{i \in I} \alpha^{i} x^{i}: \alpha^{i} \in I R_{+}, x^{i} \in X \text { for each } i \in I \text {, for each finite set } I\right\} \text {, }
$$


which is denoted by Cone $(X)$. It consists of all elements which can be expressed as a finite non-negative linear combination of elements of $X$. If we only allow non-negative weights summing up to 1 then the set is denoted by $\operatorname{Conv}(X)$ and it consists of all convex combination of elements from $X$ (we already used this notion before). If no restrictions are put on the weights then the set is denoted by $\operatorname{Span}(X)$. It is immediate that for each two sets $X, Y \subseteq I R^{m}$ we have

$$
\operatorname{Cone}(X \cup Y)=\operatorname{Cone}(X)+\operatorname{Cone}(Y) \text {. }
$$

For a subset $X$ of a cone $C$ we have $\operatorname{Cone}(X) \subseteq C$. Now $X$ is called a generating set for $C$ if $\operatorname{Cone}(X)=C$.

Examples of cones are the set $\{0\}$, with 0 denoting the origin, the (closed) half-spaces and intersections of a family of cones.

\section{Intersection of closed half-spaces}

A cone is said to be polyhedral if it is the intersection of a finite family of closed half-spaces. There are several characterizations available for polyhedral cones. We will frequently use the following one. For a proof the reader is referred to Klee [1959].

Theorem 1.11 A cone $C$ is polyhedral if and only if there is a finite subset $X$ of $C$ such that each element of $C$ is a non-negative linear combination of elements of $X$.

In other words a set $C$ is an intersection of a finite number of closed halfspaces if and only if there exist a finite generating set for $C$.

The following notations are used. A closed half-space $\left\{x \in I R^{m}: y\right.$. $x \geq 0\}$ associated to the normal vector $y \in I R^{m}$ is denoted by $\mathcal{H}(y)$. The intersection $\bigcap_{i \in I} \mathcal{K}_{i}$ is sometimes denoted by $\bigcap\left\{\mathcal{K}_{i}: i \in I\right\}$.

The following lemma plays an important role in the next chapter. For an arbitrary closed cone $D$ it characterizes all those families of closed half-spaces whose intersection equals $D$.

Lemma 1.12 For a closed cone $D \subseteq I R^{m}$ and a set $Y \subseteq I R^{m}$ we have

$$
D=\bigcap_{v \in Y} \mathcal{H}(y) \text { if and only if } \operatorname{Cone}(Y)=\left\{y \in I R^{m}: D \subseteq \mathcal{H}(y)\right\}
$$

Proof: Suppose $D=\bigcap_{y \in Y} \mathcal{H}(y)$, i.e., $D=\left\{x \in I R^{m}: y \cdot x \geq 0\right.$ for all $y \in$ $Y\}$. We obviously have $\operatorname{Cone}(Y) \subseteq\left\{y \in I R^{m}: D \subseteq \mathcal{K}(y)\right\}$. For an arbitrary 
vector $\hat{y} \notin$ Cone $(Y)$ there exist an $\hat{x} \in I R^{m}$ and a scalar $\alpha$ with $\hat{y} \cdot \hat{x}<\alpha$ and $y \cdot \hat{x} \geq \alpha$ for all $y \in$ Cone $(Y)$ according to the Separation Theorem. Since $0 \in \operatorname{Cone}(Y)$ we must have $\alpha \leq 0$. Now if there is an element $y \in \operatorname{Cone}(Y)$ with $y \cdot \hat{x}<0$ then the inequality $\lambda y \cdot \hat{x} \geq \alpha$ cannot hold for all positive scalars $\lambda$. We conclude that if $\alpha<0$ then $y \cdot \hat{x} \geq 0$ for all $y \in \operatorname{Cone}(Y)$. Of course, the latter condition also holds in case $\alpha \geq 0$. This implies that $\hat{x} \in D$ and $\hat{x} \notin \mathcal{H}(\hat{y})$; therefore $D \notin \mathcal{H}(\hat{y})$. We conclude that the inclusion $\left\{y \in I R^{m} ; D \subseteq \mathcal{H}(y)\right\} \subseteq$ Cone $(Y)$ must hold. With the already mentioned reverse inclusion we thus have Cone $(Y)=\left\{y \in I R^{m}: D \subseteq H(y)\right\}$.

Now suppose $D \neq \bigcap_{y \in Y} \mathcal{H}(y)$. In case $D \notin \mathcal{K}(y)$ for a particular $y \in Y$ it is clear that Cone $(Y)$ is unequal to $\left\{y \in I R^{m}: D \subseteq \mathcal{H}(y)\right\}$. Thus, assume that $D \subseteq \mathcal{X}(y)$ for all $y \in Y$. Let $\hat{x} \in\left(\bigcap_{\nu \in Y} \mathcal{Y}(y)\right) \backslash D$, i.e., $\hat{x} \notin D$ and $y \cdot \hat{x} \geq 0$ for all $y \in Y$. Again using the Separation Theorem there is a vector $\hat{y}$ and scalar $\alpha$ such that $\hat{y} \cdot \hat{x}<\alpha$ and $\hat{y} \cdot x \geq \alpha$ for all $x \in D$. Since $0 \in D$ we have $\alpha \leq 0$, implying $\hat{y} \cdot \hat{x}<0$. Therefore, $\hat{y} \notin \operatorname{Cone}(Y)$ since $\hat{y}$ cannot be written as a positive combination of vectors $y$ for which $y \cdot \hat{x} \geq 0$ holds, such as the elements of $Y$. We conclude that Cone $(Y) \neq\left\{y \in I R^{m}: D \subseteq H(y)\right\}$.

\subsubsection{Extreme directions}

An element $x$ of a convex set $C$ is called extreme if it cannot be written as a convex combination of two elements of $C \backslash\{x\}$; i.e.,

if $x=\alpha y+(1-\alpha) z$ for $y, z \in C$ and scalar $\alpha, 0<\alpha<1$, then $y=z=x$.

The set of extreme points of $C$ is denoted by $\operatorname{Extr}(C)$.

Consider the convex set of the following form:

$$
[a, b]=\left\{x \in I R^{m}: a \leq x \leq b\right\},
$$

for vectors $a, b \in I R^{m}$ with $a \leq b$. We call this set an interval with top element $b$ and bottom element $a$ (notice that the core cover is an intersection of an interval and a hyperplane). Elements of an interval are the so-called mixtures of $a$ and $b$ :

$$
\operatorname{mix}_{J}(a, b)= \begin{cases}a_{i} & \text { if } i \notin J \\ b_{i} & \text { if } i \in J,\end{cases}
$$

with $J \subseteq\{1,2, \ldots, m\}$ an arbitrary index set $\left(\operatorname{mix}_{J}(0, b)\right.$ is also denoted by $\left.\operatorname{mix}_{J}(b)\right)$. These elements correspond to the corner points of $[a, b]$. To be precise, 
Lemma 1.13 The extreme elements of the interval $[a, b]$, with $a, b \in \mathbb{R ^ { m }}$, $a \leq b$, are the mixtures of $a$ and $b$.

Proof: For an element $x$ which is not a mixture consider $y=x+\epsilon \chi^{\{k\}}$ and $z=x-\epsilon \chi^{\{k\}}$ where the index $k$ is such that $a_{k}<x_{k}<b_{k}$, and $\epsilon>0$ is chosen such that $y, z \in[a, b]$. Evidently, $x \neq y$ and $x=\frac{1}{2} y+\frac{1}{2} z$, implying that $x$ cannot be extreme. This implies that the extreme elements of $[a, b]$ are among the mixtures of $a$ and $b$. Now suppose mix $J(a, b)$ is a convex combination of $\left.x, y \in[a, b], \operatorname{mix}_{J}(a, b)\right)=\alpha x+(1-\alpha) y$, with $0<\alpha<1$. From

$$
x_{j} \leq b_{j}, y_{j} \leq b_{j} \text { and } \alpha x_{j}+(1-\alpha) y_{j}=\left(\operatorname{mix}_{J}(a, b)\right)_{j}=b_{j} \text { for } j \in J,
$$

we conclude that $x_{j}=y_{j}=b_{j}, j \in J$, and from

$$
x_{j} \geq a_{j}, y_{j} \geq a_{j} \text { and } \alpha x_{j}+(1-\alpha) y_{j}=\left(\operatorname{mix}_{J}(a, b)\right)_{j}=a_{j} \text { for } j \notin J,
$$

it follows that $x_{j}=y_{j}=a_{j}, j \notin J$. Therefore, $x=y=\operatorname{mix}_{J}(a, b)$, which implies the extremality of $\operatorname{mix}_{J}(a, b)$.

For an element $x$ of the interval $[a, b]$ the following expression denotes a decomposition of $x$ into a convex combination of mixtures of $[a, b]$ (without loss of generality assume that $a<b$ ):

$$
x=\sum_{r=1}^{m+1}\left(\alpha_{\pi(r)}-\alpha_{\pi(r-1)}\right) \operatorname{mix}_{\{\pi(1), \ldots, \pi(r-1)\}}(a, b),
$$

with scalars $\alpha_{0}=0, \alpha_{m+1}=1$ and $\alpha_{r}=\left(x_{r}-a_{r}\right)\left(b_{r}-a_{r}\right)^{-1}$ for $r \epsilon$ $\{1,2, \ldots, m\}$, and $\pi$ a permutation on $\{0,1, \ldots, m+1\}$ such that $\alpha_{\pi(0)} \leq$ $\alpha_{\pi(1)} \leq \ldots \leq \alpha_{\pi(m+1)}$ (one may choose $\pi(0)=0$ and $\pi(m+1)=m+1$ ). The expression (1.19) holds since for each $k \in\{1,2, \ldots, m\}$ we have (without loss of generality we let $\pi$ to be the identity permutation)

$$
\begin{aligned}
\left(\sum_{r=1}^{m+1}\left(\alpha_{r}-\alpha_{r-1}\right) \operatorname{mix}_{\{1, \ldots, r-1\}}(a, b)\right)_{k} & \\
& \left.=\sum_{r=1}^{m+1}\left(\alpha_{r}-\alpha_{r-1}\right)\left(\operatorname{mix}_{\{1, \ldots, r-1\}}(a, b)\right)\right)_{k} \\
& =\sum_{r=1}^{k}\left(\alpha_{r}-\alpha_{r-1}\right) b_{k}+\sum_{r=k+1}^{m+1}\left(\alpha_{r}-\alpha_{r-1}\right) a_{k}
\end{aligned}
$$




$$
\begin{aligned}
& =\left(\alpha_{k}-\alpha_{0}\right) b_{k}+\left(\alpha_{m+1}-\alpha_{k}\right) a_{k} \\
& =\alpha_{k}\left(b_{k}-a_{k}\right)+a_{k} \\
& =x_{k}+a_{k}+a_{k} \\
& =x_{k}
\end{aligned}
$$

\section{Pointedness}

For a non-empty cone only one element, the origin, may be extreme. In the case the origin is extreme the cone is called pointed. Obviously, the pointedness of a non-empty cone $C$ is equivalent to $C \cap(-C)=\{0\}$ (here $-C$ denotes the set $\{-x: x \in C\})$.

Extreme directions of a cone

An element $x \neq 0$ of a cone $C$ is called extreme or an extreme direction if the following holds:

$$
\text { if } x=y+z \text { for } y, z \in C \text { then } y, z \in \operatorname{Cone}(\{x\}) \text {. }
$$

The set of extreme directions is denoted by $\operatorname{EDir}(C)$. Clearly, there exists no extreme directions if $C$ is not pointed, since in that case it contains a non-trivial linear subspace as a subset. If $\operatorname{EDir}(C) \neq\{0\}$ it consists of a union of half-lines starting at the origin. A subset $E$ of extreme directions is called representative if each $x \in \operatorname{EDir}(C)$ is a positive multiple of one and only one element in $E$.

\section{Extreme directions and generating sets}

There is a close relationship between the notions of extreme direction and generating set. One easily shows that $(1.20)$ is equivalent to the property

$$
X \cap \operatorname{Cone}(\{x\}) \neq \emptyset \text { for each generating set } X \subseteq C .
$$

Furthermore, $\operatorname{EDir}(C)$ and each representative set are generating sets for a closed and pointed cone $C$. This can be derived from the following theorem (a proof can be found in Klee [1959]).

Theorem 1.14 Let $C$ be a closed and pointed cone, and $X \subseteq C \backslash\{0\}$. Then $X$ is a generating set of $C$ if and only if $X \cap C o n e(\{x\}) \neq \emptyset$ for each extreme direction $x$ of $C \backslash\{0\}$.

Thus, the generating sets of a closed and pointed cone are exactly the subsets containing a reprentative subset of extreme directions as a subset.

The following theorem can now easily be derived. It describes a characterization of generating sets. 
Theorem 1.15 Let $C$ be a closed and pointed cone; and $X \subseteq C$. Then $X$ is a generating set of $C$ if and only if for each $y \in C \backslash\{0\}$ there is an element $x \in X$ and a positive scalar $\alpha$ such that $y-\alpha x \in C$.

Proof:

('if'-part) The latter statement of the theorem implies that $X$ contains a positive multiple of each extreme direction. Using theorem 1.14 we conclude that $X$ is a generating set.

('only if'-part) The 'only if'-part follows immediately from the definition of a generating set.

The reader is referred to Klee [1959] and Rockafellar [1970] for more detailed information about these concepts.

\subsubsection{Cones of games}

The inequality conditions in section 1.2 give rise to closed half-spaces in $\mathbf{G}^{N}$. For example the monotonicity conditions in (1.1) are equivalent to

$$
v \in \mathcal{H}\left(\mathbb{1}^{T}-\mathbf{1}^{S}\right) \text { for all non-empty coalitions } S \subseteq T \text {. }
$$

This implies that the class $\mathbf{M N}^{N}$ of monotonic games is a polyhedral cone in $\mathbf{G}^{N}$.

Also the (total) balancedness conditions can be treated in a similar way. A vector $\lambda \in I R^{2^{N} \backslash\{0\}}$ is called balanced if

$$
\lambda_{N} \geq 0, \lambda_{S} \leq 0, \text { for } S \subset N \text {, and } \sum_{S \ni i} \lambda_{S}=0, \text { for each } i \in N,
$$

and $\lambda$ is called totally balanced if

there is a $T \subseteq N$ with $\lambda_{T} \geq 0, \lambda_{S} \leq 0$, for $S \subset T, \lambda_{S}=0$, for $S \not \subset T$,

$$
\text { and } \sum_{S \ni i} \lambda_{S}=0 \text {, for each } i \in T \text {. }
$$

Notice that $\lambda \leq 0$ for a (totally) balanced vector implies $\lambda=0$. For a balanced vector with $\lambda_{N}>0$ the family $B=\left\{S \subset N: \lambda_{S}<0\right\}$ of coalitions is balanced with balanced collection $\left(-\lambda_{S} / \lambda_{N}\right)_{S \in B}$. Moreover, for a balanced collection there obviously exists a corresponding balanced vector in the above way. We conclude that the (total) balancedness property of a game $v$ is equivalent to

$$
\lambda \cdot v=\sum_{S \subseteq N} \lambda_{S} v(S) \geq 0 \text { for each (totally) balanced vector } \lambda .
$$


The classes $\mathbf{B}^{N}$ and $\mathbf{T B}^{N}$ are therefore closed cones.

In a similar way one proves the cone property of other introduced classes of games.

\section{Linear mappings}

Let $f: D \longrightarrow \mathrm{G}^{N}$ be a linear mapping on a cone $D=\operatorname{Cone}(X)$. The image $f(D)$ is again a cone, which is generated by the images of the elements in $X$, and if $x$ is extreme in $D$ and $f(x) \neq 0$ then $f(x)$ is extreme in $f(D)$.

For an allocation $x \in I R^{N}$ let $u^{x}$ denote the corresponding additive game defined by

$$
u^{x}(S)=\sum_{i \in S} x_{i} \text { for each non-empty coalition } S
$$

Now $u$, considered as a mapping on the allocation space $I R^{N}$, is linear and defines an isomorphism between $I R^{N}$ and the class $\mathrm{A}^{N}$ of additive games. The allocations $\chi^{\{i\}}, i \in N$ span $I R^{N}$, i.e., $I R^{N}=\operatorname{Span}\left(\left\{\chi^{\{i\}}: i \in N\right\}\right)$. The additive games corresponding to these allocations are the dictator games. We, thus, have

Lemma 1.16 The class $\mathbf{A}^{N}$ of additive games equals $\operatorname{Span}\left(\left\{u^{\{i\}}: i \in N\right\}\right)=$ Cone $\left(\left\{u^{\{i\}}: i \in N\right\} \cup\left\{-u^{\{i\}}: i \in N\right\}\right)$.

In the following two theorems we consider a generalized version of the above observations.

Theorem 1.17 Let $f: D \longrightarrow \mathbf{G}^{N}$ be a linear mapping on a non-empty cone $D$, and let $\Omega \subseteq 2^{N} \backslash\{0\}$ denote an arbitrary non-empty set of coalitions. Consider the cone $\mathbf{W}^{N}$ of games $v \in \mathbf{G}^{N}$ such that

there is an $x \in D$ with $v \leq f(x)$, and $v=f(x)$ on the coalitions in $\Omega$.

The cones $\mathbf{W}^{N}, \mathbf{W}_{0}^{N}$ and $\mathbf{W}_{+}^{N}$ are characterized as follows (the game $f(x)$ is sometimes denoted by $f^{*}$ ).

A: Let $X \subseteq D$ be a generating set of $D$. The class $\mathrm{W}^{N}$ of games is a cone generated by the games $f(x), x \in X$, and $-1{ }^{S}, \emptyset \neq S \in 2^{N} \backslash \Omega$.

B: Let $X_{z} \subseteq D$ be a generating set of the cone $D_{z}=\left\{x \in D: f^{x}(\{i\}) \geq 0\right.$ for all $i \in N\}$. The class $\mathbf{W}_{0}^{N}$ of the O-normalized games in $\mathbf{W}^{N}$ is a cone generated by the games $f(x)-\sum_{j \in N} f^{x}(\{j\}) \mathbb{1}^{\{j\}}, x \in X_{z}$, and $-\mathbf{1}^{S}, S \in 2^{N} \backslash \Omega,|S| \geq 2$. 
C: Let $X_{p} \subseteq D$ be a generating set of the cone $D_{p}=\left\{x \in D: f(x) \in \mathbf{G}_{+}^{N}\right\}$. The class $\mathbf{W}_{+}^{N}$ of the non-negative games in $\mathbf{W}^{N}$ is a cone generated by the games $\operatorname{mix}_{\Omega^{\prime}}(f(x))$, with $x \in X_{p}$ and $\Omega^{\prime} \subseteq 2^{N} \backslash\{\emptyset\}, \Omega \subseteq \Omega^{\prime}$.

Proof:

Proof of part $\mathbf{A}$ : let $v \in \mathbf{W}^{N}$ and $x \in D$ be such that (1.23) holds. Let $x$ equal the non-negative weighted sum $\sum_{y \in X} \alpha^{y} y$. Then

$$
\begin{aligned}
v & =f(x)-(f(x)-v) \\
& =f\left(\sum_{y \in X} \alpha^{y} y\right)+\sum_{\emptyset \neq S \subseteq N}\left(f^{x}(S)-v(S)\right)\left(-\mathbf{1}^{S}\right) \\
& =\sum_{y \in X} \alpha^{y} f(y)+\sum_{\emptyset \neq S \subseteq N, S \notin \Omega}\left(f^{x}(S)-v(S)\right)\left(-\mathbb{1}^{s}\right) .
\end{aligned}
$$

Observe that the games $f(y), y \in X$, are contained in $\mathbf{W}^{N}$. Also the games $-\mathbb{1}^{S}$ for the non-empty coalitions $S \notin \Omega$ are elements of $\mathbf{W}^{N}$, since these games fulfil (1.23) (with $x=0$ ). We conclude that part $\mathbf{A}$ holds.

Proof of part $\mathbf{B}$ : A game $v \in \mathbf{G}_{0}^{N}$ is contained in $\mathbf{W}_{0}^{N}$ if and only if it fulfils (1.23). Since $v(\{i\})=0$ for all $i \in N$ we may replace $D$ in (1.23) by $D_{z}$. Let $W$ denote the set of the games mentioned in part $\mathbf{B}$. By a similar reasoning as in the proof of part $\mathbf{A}$ it now follows that $W$ is a generating set of $\mathbf{W}_{0}^{N}$. Proof of part C: Let $W$ now denote the games mentioned in part $\mathbf{C}$. It is an easy exercise to check that these games are indeed contained in $\mathbf{W}_{+}^{N}$. We will first show that a game $v$ in $\mathbf{W}_{+}^{N}$ can be represented by a non-negative weighted sum $\sum_{y \in X_{p}} \alpha^{y} v^{y}$ of games $v^{y}$ with $v^{\nu} \leq f(y)$ and $v^{y}=f(y)$ on the coalitions in $\Omega$. The game $v$ fulfils (1.23), say with $x \in D$ chosen to be $x^{0}$. Of course, $x^{0}$ must be an element of $D_{p}$; then $x^{0}$ is a non-negative weighted sum $\sum_{y \in X_{\mathrm{p}}} \alpha^{y} y$ of elements of $X_{\mathrm{p}}$. If for a coalition $S$ we have $0=f^{x}(S)=\sum_{y \in X_{p}} \alpha^{y} f^{y}(S)$ then also $f^{y}(S)=0$ for all $y \in X_{p}$ with $\alpha^{y} \neq 0$. Now define the game $v^{v}$ to be equal to 0 for the coalitions $S$ with $f^{x}(S)=0$ and $\left(f^{y}(S) / f^{z}(S)\right) v(S)$ otherwise. Then $v(S)=0=\sum_{\nu \in X_{p}} \alpha^{y} v^{\nu}(S)$ for coalitions $S$ with $f^{x}(S)=0$ and

$$
\sum_{y \in X_{p}} \alpha^{y} v^{y}(S)=\frac{\sum_{y \in X_{p}} \alpha^{y} f^{v}(S)}{f^{x}(S)} v(S)=\frac{f^{x}(S)}{f^{x}(S)} v(S)=v(S)
$$

for the other coalitions. We conclude that $v=\sum_{v \in X_{p}} \alpha^{y} v^{v}$.

The game $v^{v}$, with $y \in X_{p}$, is an element of the interval $[0, f(y)]$. We showed in lemma 1.13 that the extreme elements of this convex set equals $\operatorname{mix}_{\Omega^{\prime}}(0, f(y)), \Omega^{\prime} \subseteq 2^{N}$. Since $v^{y}$ equals $f(y)$ on $\Omega$ we conclude that $v^{y}$ is 
a convex combination of the games $\operatorname{mix}_{\Omega^{\prime}}(f(y))$, with $\Omega \subseteq \Omega^{\prime} \subseteq 2^{N}$. These games are contained in $\mathbf{W}_{+}^{N}$, which shows that the games $v^{y}, y \in X_{p}$, are contained in Cone $(W)$. Then also $v \in \operatorname{Cone}(W)$, i.e., $W$ is a generating set of $D_{p}$.

A linear mapping $f: D \longrightarrow \mathbf{G}^{N}$ is called $\Omega$-injective, with $\Omega \subseteq 2^{N}$, if

$$
f(x)=f(y) \text { on the coalitions in } \Omega \text { implies } x=y \text {. }
$$

Notice that $\Omega$-injectivity implies that $f$ cannot be zero on the coalitions in $\Omega$.

Theorem 1.18 Consider the cone $\mathbf{W}^{N}$ of games as described in theorem 1.17. Assume that $f$ is $\Omega$-injective. Then

A: Suppose $D_{z}$ is pointed. The games $-1^{S}, S \in 2^{N} \backslash \Omega$ and $|S| \geq 2$, are extreme in $\mathbf{W}_{0}^{N}$. Furthermore, the game $f(x)-\sum_{j \in N} f^{x}(\{j\}) \mathbb{1}^{\{j\}}$, is an extreme direction of $\mathrm{W}_{0}^{N}$ if $x$ is extreme in $D_{z}$.

B: Suppose $D_{p}$ is pointed. The game mix $\Omega_{\Omega^{\prime}}(f(x))$, with $x \in D_{p}$ extreme, and $\Omega^{\prime} \subseteq 2^{N} \backslash\{\emptyset\}, \Omega \subseteq \Omega^{\prime}$, is an extreme direction of $\mathbf{W}_{+}^{N}$.

The proofs of part $\mathbf{A}$ and part $\mathbf{B}$ will be done simultaneously. We will prove part $\mathbf{A}$, and the adjustments are specified within parentheses, which are needed for the proof of part $\mathbf{B}$.

Proof: Let $v$ be one of the games mentioned in part $\mathbf{A}$ (in part B). Suppose $v=v^{\prime}+v^{\prime \prime}$, with $v^{\prime}, v^{\prime \prime} \in \mathbf{W}_{0}^{N}\left(v^{\prime}, v^{\prime \prime} \in \mathbf{W}_{+}^{N}\right)$. We will show that $v^{\prime}$ and $v^{\prime \prime}$ are non-negative multiples of $v$, which proves that $v$ is extreme in $\mathbf{W}_{0}^{N}$ (in $\mathbf{W}_{+}^{N}$ ). The games $v^{\prime}$ and $v^{\prime \prime}$ fulfil (1.23), say with $x \in D_{z}\left(x \in D_{p}\right)$ chosen to be $x^{0}, x^{\prime}$ and $x^{\prime \prime}$ respectively. We may choose $x^{0}$ to be an element of $X_{z} \cup\{0\}$ (of $X_{p}$ ), where $X_{z}$ (where $X_{p}$ ) consists of extreme directions of $D_{x}\left(\right.$ of $\left.D_{p}\right)$. Then $f\left(x^{0}\right)=v=v^{\prime}+v^{\prime \prime}=f\left(x^{\prime}\right)+f\left(x^{\prime \prime}\right)=f\left(x^{\prime}+x^{\prime \prime}\right)$ on the coalitions in $\Omega$, implying $x^{0}=x^{\prime}+x^{\prime \prime}$ according to the fact that $f$ is $\Omega$-injective. If $x^{0}=0$ then $v=-1^{S}$ for a non-empty coalition $S \notin \Omega$. From $x^{\prime}+x^{\prime \prime}=x^{0}=0$ and the pointedness of $D_{z}$ it follows that also $x^{\prime}=x^{\prime \prime}=0$. Therefore, $v^{\prime} \leq f(0)=0$, i.e., $v^{\prime}$ is majorized by the all-losing game, and $v^{\prime \prime} \leq 0$. From $-\mathbf{1}^{S}=v=v^{\prime}+v^{\prime \prime}$ it now follows that $v^{\prime}$ and $v^{\prime \prime}$ are nonnegative multiples of $v$.

Thus, suppose now that $x^{0} \neq 0$. Then $v$ equals $f\left(x^{0}\right)-\sum_{j \in N} f^{x^{0}}(\{i\}) \mathbf{1}^{\{i\}}$ $\left(v=\operatorname{mix}_{\Omega^{\prime}}\left(f\left(x^{0}\right)\right)\right.$, for an $\left.\Omega^{\prime} \subseteq 2^{N} \backslash\{\theta\}, \Omega \subseteq \Omega^{\prime}\right)$. Now $x$ is an extreme direction of $D_{z}$ (of $\left.D_{p}\right)$, which implies that $x^{\prime}$ and $x^{\prime \prime}$ are non-negative multiples 
of $x$, say $x^{\prime}=\alpha^{\prime} x$ and $x^{\prime \prime}=\alpha^{\prime \prime} x$. Observe that from $v^{\prime} \leq f\left(x^{\prime}\right)=\alpha^{\prime} f(x)$, $v^{\prime \prime} \leq \alpha^{\prime \prime} f(x)$ and the 0-normalizedness of $v, v^{\prime}$ and $v^{\prime \prime}$, (and $v(S)=0=$ $v^{\prime}(S)=v^{\prime \prime}(S)$ for coalitions $S$ with $v(S)<f^{x}(S)$,) it follows that $v^{\prime} \leq \alpha^{\prime} v$ and $v^{\prime \prime} \leq \alpha^{\prime \prime} v$. Furthermore, there is a coalition $S$ in $\Omega$ with $f^{z}(S) \neq 0$. Then $f^{x}(S)=v(S)=v^{\prime}(S)+v^{\prime \prime}(S)=\alpha^{\prime} f^{x}(S)+\alpha^{\prime \prime} f^{x}(S)$ implying $\alpha^{\prime}+\alpha^{\prime \prime}=1$. Thus, $v^{\prime}+v^{\prime \prime}=v=\alpha^{\prime} v+\alpha^{\prime \prime} v \geq v^{\prime}+v^{\prime \prime}$, and we conclude that $v=\alpha^{\prime} v^{\prime}$ and $v=\alpha^{\prime \prime} v^{\prime \prime}$ must hold.

Applications of these theorems will be discussed in the following chapters.

\section{Decompositions into the classes of additive and 0-normalized games}

For most of the considered classes of games the class $\mathbf{A}^{N}$ is a subset. The following lemma states that each of these classes can be decomposed into the algebraic sum of the class of additive games and the class of the 0-normalized games.

Lemma 1.19 Let $\mathrm{W}^{N}$ be a cone of games containing the class $\mathrm{A}^{N}$ of additive games. Then $\mathbf{W}^{N}$ is the algebraic sum of $\mathbf{A}^{N}$ and the class $\mathbf{W}_{0}^{N}$ of the 0 -normalized games in $\mathbf{W}^{N}$. Furthermore, we have $\mathbf{W}_{+}^{N}=\mathbf{A}_{+}^{N}+\mathbf{W}_{0}^{N}$ if

$$
v(S) \geq \sum_{i \in S} v(\{i\}) \text { for each non-empty coalition } S,
$$

for all games $v \in \mathbf{W}^{N}$.

Proof: Obviously, $\mathbf{A}^{N}+\mathbf{W}_{0}^{N} \subseteq \mathbf{W}^{N}$. Now let $w \in \mathbf{W}^{N}$ and $u$ denote the additive game associated to $(w(\{i\}))_{i \in N}$. Then $w_{0}=w-u \in \mathbf{W}^{N}+\mathbf{A}^{N}=$ $\mathbf{W}^{N}$ yielding $w_{0} \in \mathbf{W}_{0}^{N}$. Therefore, $w=w_{0}+u \in \mathbf{A}^{N}+\mathbf{W}_{0}^{N}$. We conclude that $\mathbf{W}^{N}$ equals $\mathbf{A}^{N}+\mathbf{W}_{0}^{N}$.

The last assertion follows in a similar way from the observation that (1.25) implies the non-negativity of a game in $\mathbf{W}_{0}^{N}$.

Observe that for a cone $\mathbf{W}^{N}$ for which $\mathbf{A}^{N}$ is the maximal linear subspace the cone $\mathbf{W}_{0}^{N}$ must be pointed.

In a game theoretical context the extremality of a game in a (not necessarily pointed) cone $\mathbf{W}^{N}$ of games could have been defined as: a game $v \in \mathbf{W}^{N}$ is extreme if $v=v^{1}+v^{2}$, with $v^{1}, v^{2} \in \mathbf{W}^{N}$, implies that both $v^{1}$ and $v^{2}$ are strategically equivalent with $v$. This definition, however, corresponds exactly to the extremality of the 0-normalization of $v$ in $\mathbf{W}_{0}^{N}$.

\section{Generating sets of simple games}

Let us conclude this section with some results concerning generating sets of simple games. 
Lernma 1.20 Let $\mathbf{W}^{N} \subseteq \mathrm{G}^{N}$ be a cone of games for which its simple games form a generating set. Then $\mathbf{W}^{N}$ is pointed and its extreme directions are precisely the positive multiples of the simple games in $\mathbf{W}^{N}$.

Proof: The games in $\mathbf{W}^{N}$ are non-negative linear combinations of simple games and therefore elements of the pointed cone $\mathbf{G}_{+}^{N}$. This implies the pointedness of $\mathbf{W}^{N}$.

The only possible extreme games are the positive multiples of the simple games in $\mathbf{W}^{N}$. Thus, we conclude the proof by showing that a simple game $w$ of $\mathbf{W}^{N}$ is extreme. Suppose that $w$ equals $v+u$ for two games $v, u \in$ $\mathbf{W}^{N} \backslash\{0\}$. The games $v$ and $u$ are positive linear combinations of simple games, say $v=\sum_{r=1}^{q} \lambda^{r} w^{r}$ and $u=\sum_{r=q+1}^{Q} \lambda^{r} w^{r}$, with $w^{r} \in \mathbf{W}^{N}$ for $1 \leq$ $r \leq Q$. If $w(S)=0$ for a coalition $S$ then $\lambda^{r} w^{r}(S)=0$, which implies $w^{r}(S)=0$ for all $r \in\{1,2, \ldots, Q\}$; and if $w(S)=1$ then

$$
w(S)=1=\sum_{r=1}^{Q} \lambda^{r} w^{r}(S) \leq \sum_{r=1}^{Q} \lambda^{r}=\sum_{r=1}^{Q} \lambda^{r} w^{r}(N)=w(N)=1,
$$

yielding $w^{r}(S)=1$ for all $r \in\{1,2, \ldots, Q\}$. We conclude that all involved simple games are equal. Thus, $v$ and $u$ are non-negative multiples of $w$. This proves the extremality of $w$ in $\mathbf{W}^{N}$.

A similar reasoning can be used in order to prove

Lemma 1.21 Let $\mathbf{W}^{N} \subseteq \mathbf{G}^{N}$ be the convex hull of the simple games in $\mathbf{W}^{N}$. Then the extreme elements of $\mathbf{W}^{N}$ are precisely its simple games.

As we shall see later on several classes of games are generated by their simple games. As an example consider the class $\mathbf{M N}_{+}^{N}$ of non-negative monotonic games. The presented proof is constructive in nature, i.e., we will describe an arbitrary non-negative monotonic game $v \neq 0$ as a positive weighted sum of monotonic simple games.

Theorem 1.22 The class $\mathbf{M N}_{+}^{N}$ is generated by the set of monotonic simple games. These games are extreme directions of $\mathbf{M N}_{+}^{N}$.

Proof: Let $0=v(\theta)=\lambda^{0}<\lambda^{1}<\ldots<\lambda^{R}=v(N)$ be the worths of the coalitions in the game $v$. Define the simple games $w^{1}, w^{2}, \ldots, w^{R}$ as follows

$$
w^{r}(S)=\left\{\begin{array}{cc}
1 & \text { if } v(S) \geq \lambda^{r}, \\
0 & \text { otherwise, }
\end{array} \quad \text { with } S \subseteq N \text { and } 1 \leq r \leq R .\right.
$$


Observe that the games $w^{r}, 1 \leq r \leq R$, are simple. The monotonicity of these games clearly follows from the definition. Furthermore,

$$
v=\sum_{r=1}^{R}\left(\lambda^{r}-\lambda^{r-1}\right) w^{r}
$$

This proves that $\mathbf{M N}_{+}^{N}$ is generated by the monotonic simple games. The extremality of these games in $\mathbf{M N}_{+}^{N}$ follows from lemma 1.20 . 


\section{Chapter 2}

\section{Cones of games with a non-empty core}

\subsection{Introduction}

Our first concern in this chapter is the characterization of several classes of games with a non-empty core, considered as cones in the game space. This will be done by presenting generating sets. The main result is the characterization of the extreme directions of the class $\mathbf{C}_{+}^{N}$ of non-negative games with a non-empty core: its extreme games correspond to the vetocontrolled simple games. A constructive method is given in order to represent a game as a weighted sum of veto-controlled simple games.

As an application of our main result an alternative proof is presented of the well-known equivalence of the balancedness property and the nonemptiness of the core.

Also other applications concerning linear programming games and flow games are discussed.

The contents

In section 2.2 we will start with a discussion of a generating set for the cone $\mathbf{C}^{N}$. Furthermore, some subsets of $\mathbf{C}^{N}$ are treated, such as the set of the 0 -normalized games with a non-empty core and the set of games for which the marginal contribution allocation is an element of the core. In subsection 2.2.3 we will give a new proof of the BondarevarShapley correspondence, expressing the equivalence between the non-emptiness of the core and the balancedness. In the proof we will employ the results of subsection 2.2.1. The subsections 2.2.4, 2.2.5 and 2.2 .6 are devoted to the set $\mathbf{C}_{+}^{N}$ of non- 
negative games with a non-empty core. The generating set which we will present for this pointed cone consists of the simple veto-controlled games. It is shown that these games are extreme in $\mathbf{C}_{+}^{N}$. On the basis of these results we will show again the Bondareva-Shapley correspondence restricted to the non-negative games. Furthermore, a method is discussed to represent a non-negative game as a non-negative weighted sum of simple veto-controlled games. We end section 2.2 with a discussion of two subclasses of $\mathbf{C}_{+}^{N}$ : the class of games having a so-called population monotonic allocation scheme and the class of games generated by the airport cost savings games.

The remaining sections are devoted to three subjects of cooperative game theory in which the core and the balancedness are central notions. Along with a detailed discussion of these subjects the results of the previous sections are applied.

In section 2.3 the so-called linear programming games are studied. These games are introduced as arising from a linear production model in which the resources are controlled by a set of players. Using the characterization of the non-negative games with a non-empty core in section 2.2 it is shown that two apparently different generalizations of the controlled linear production model, as discussed in literature, in fact yield the same model. In addition we will present a class of linear programming games with a non-empty core, which is essentially larger than the one known thus far. With the help of a simple argument we show that not all core allocations of a linear programming game need to correspond to dual optimal solutions of the linear programming problem, related to the computation of the worth of the grand coalition.

Section 2.4 discusses the so-called multicommodity game. It is a generalization of our notion of a game and has a natural application in controlled linear production models. We will characterize the controlled linear production models which give rise to a balanced multicommodity game.

The concluding section of this chapter is devoted to the subject of flow games. We will introduce a multicommodity transportation model in which flow is controlled by a set of players. From this model the so-called (multicommodity) flow game is derived. In the one-commodity case there is a nice result characterizing the flow games as being the non-negative totally balanced games. In the multicommodity case, however, we will show that although a multicommodity flow game is balanced, it is not possible to represent each balanced two-commodity game as a flow game. 


\subsection{Cones of games with a non-empty core}

The core considered as a set-valued function on the game space has the so-called superadditivity property

$$
\operatorname{Core}(v)+\operatorname{Core}(w) \subseteq \operatorname{Core}(v+w) \text { for all games } v, w \in \mathbf{G}^{N}
$$

(where an algebraic sum is assumed to be empty if one of the summands is empty). See also Peleg [1986] where an axiomatic characterization is given of the core in which the superadditivity plays a significant role. The core is in fact additive on the class of convex games (see Dragan, Potters and Tijs [1989]). Additivity does not hold in general. To show this, observe that normally the core consists of more than one allocation: in the unity game $\mathbb{1}^{N}$, for example, all individual rational allocations $x \in I R_{+}^{N}$, with $\sum_{j \in N} x_{j}=1$, belong to the core, i.e., $\operatorname{Imp}\left(\mathbf{1}^{N}\right)=\operatorname{Core}\left(\mathbf{1}^{N}\right)$. Now consider an arbitrary game $v$ with a core allocation $x$. Of course, $v$ is the sum of the additive game $u^{x}$ corresponding to $x$ and the difference $v-u^{x}$. The game $v-u^{x}$ is non-positive with zero value for the grand coalition. Therefore, it is a sum of the games $\left(\sum_{j \in S} x_{j}-v(S)\right)\left(-\mathbf{1}^{S}\right), S \subset N, S \neq \emptyset$; for the game $-\mathbb{1}^{S}$ the zero allocation is the only core allocation. Also $u^{x}$ has only one core allocation, $\operatorname{Core}\left(u^{x}\right)=\{x\}$. Thus, we have written $v$ as a sum of games with cores consisting of only one allocation. The algebraic sum of these cores obviously equals $\{x\}$, in general a proper subset of Core $(v)$.

We emphasize that the results concerning the classes $\mathbf{C}^{N}, \mathbf{C}_{0}^{N}$ and $\mathbf{C}_{+}^{N}$, which are presented in the sequel, can be derived by using theorem 1.17. For the linear mapping $f$ in 1.17 one has to take the function $x \mapsto u^{x}$, with domain the allocation space $C=I R^{N}$. Furthermore, the set $\Omega$ of coalitions has only to contain one element in this case: $\Omega=\{N\}$ (notice that $f$ is not $\Omega$-injective here). One easily verifies that $C_{z}=C_{p}=I R_{+}^{N}$. However, the proofs we present here will be slightly different. In the characterizations of $\mathbf{C}^{N}$ and $\mathbf{C}_{0}^{N}$ we will use direct arguments, and in the case of $\mathbf{C}_{+}^{N}$ the proof follows similar arguments as used in the proof of theorem 1.17 but in a slightly other setting.

\subsubsection{The cone of games with a non-empty core}

Let us rephrase the above decomposition. For a game $v$ and efficient allocar tion $x$ we have

$$
v=u^{x}-\left(u^{x}-v\right)
$$




$$
\begin{aligned}
= & \sum_{j \in N} \max \left(x_{j}, 0\right) u^{(j)}+\sum_{j \in N} \max \left(-x_{j}, 0\right)\left(-u^{\{j\}}\right) \\
& +\sum_{\emptyset \neq N}\left(\sum_{j \in S} x_{j}-v(S)\right)\left(-\mathbf{1}^{S}\right) .
\end{aligned}
$$

Of course, the decomposition is a non-negative weighted sum if and only if $x$ is a core allocation of $v$. This leads to the observation that $\mathbf{C}^{N}$ is generated by the dictator games $u^{\{i\}}, i \in N$, the negatives of these games, $-u^{\{i\}}$, $i \in N$, and the negatives of the unity games, except $-\mathbb{1}^{N}$. From this simple reasoning it already follows that $\mathbf{C}^{N}$ is a polyhedral cone since it is finitely generated (cf. theorem 1.11). Furthermore, a generating set consisting of games for which only one core allocation is available.

Theorem 2.1 The cone $\mathbf{C}^{N}$ of games with a non-empty core is generated by the $2^{|N|}+2|N|-2$ games $u^{\{i\}},-u^{\{i\}}, i \in N$, and $-\mathbf{1}^{S}$, with $\neq S \subset N$.

Notice that a decomposition of a game as a positive weighted sum of games mentioned in theorem 2.1 may not be unique since different decompositions evolve from (2.1) when two non-equal core allocations are used. The generating games in theorem 2.1, however, form a minimal generating set in the sense that no games can be left out without losing the property that it has to generate $\mathbf{C}^{N}$. To show this, let $v$ be one of the generating games and suppose that it equals $\sum_{j \in N} \alpha_{j} u^{\{j\}}+\sum_{\emptyset \neq S \subset N} \alpha_{S}\left(-\mathbf{1}^{S}\right)$, with $\alpha_{i} \in I R$, $i \in N$, and $\alpha_{S} \in I R_{+}, \emptyset \neq S \subset N$. Observe that $\left(\alpha_{i}\right)_{i \in N}$ is a core allocation of $v$. Thus,

- in the case $v$ equals $-\mathbb{1}^{T}$, with $T \subset N$, then $\alpha_{i}=0$ for each $i \in N$, implying $-1^{T}=\sum_{\emptyset \neq S \subset N} \alpha_{S}\left(-1^{S}\right)$; this is only possible if $\alpha_{T}=1$ and the other scalars are zero. We conclude that none of the games $-\mathbf{1}^{S}$, $\emptyset \neq S \subset N$, can be left out.

- In the case $v$ equals $u^{\{j\}}$, with $j \in N$, we have $\left(\alpha_{i}\right)_{i \in N}=\chi^{\{j\}}$, i.e., $\alpha_{j}=1$ and $\alpha_{i}=0$ for $i \in N \backslash\{j\}$. This implies that $u^{\{j\}}=u^{\{j\}}+$ $\sum_{\emptyset \neq S \subset N} \alpha_{S}\left(-1^{S}\right)$ which is only possible if $\alpha_{S}=0$ for all $\emptyset \neq S \subset$ $N$. We conclude that all dictator games are needed and by a similar reasoning also all games $-u^{\{i\}}, i \in N$, are indispensable in order to generate the whole class $\mathbf{C}^{N}$ of games with a non-empty core.

Notice that the set $\left\{u^{(i)}: i \in N\right\} \cup\left\{-\mathbb{1}^{S}: S \subset N,|S| \geq 2\right\}$ of games with a non-empty core is in fact a base of the $\left(2^{|N|}-1\right)$-dimensional game space since it consists precisely of $2^{|N|}-1$ games and each game can be 
represented as a weighted sum of these games according to (2.1) (see also subsection 3.6.2 regarding bases of $\mathbf{G}^{N}$ ). Therefore, $\mathrm{C}^{N}$ is a ful dimensional subset of $\mathrm{G}^{N}$.

\section{Two subelasses of $\mathbf{C}^{N}$}

The decomposition in (2.1) is also useful in the determination of generating sets for the subclass $\mathbf{D}^{N}$ of $\mathbf{C}^{N}$ consisting of those games for which the marginal contribution allocation is a core allocation, and the subclass $\mathrm{H}^{N} \subset \mathbf{C}^{N}$ of games $v$ with $(v(\{i\}))_{i \in N} \in \operatorname{Core}(v)$, i.e.,

$$
\begin{aligned}
& \mathbf{D}^{N}=\left\{v \in \mathbf{G}^{N}: b^{v} \in \operatorname{Core}(v)\right\} \\
& \mathbf{H}^{N}=\left\{v \in \mathbf{G}^{N}:(v(\{i\}))_{i \in N} \in \operatorname{Core}(v)\right\} .
\end{aligned}
$$

These classes of games with a non-empty core will play an important role in the decompositions of classes of semibalanced games in chapter 3. As a result of the fact that the marginal core $\operatorname{MCore}(v)=\left\{x \in I R^{N}: x \leq b^{v}\right.$ and $\left.\sum_{j \in N} x_{j}=v(N)\right\}$ of a game $v$ contains all core allocations, the core consists only of $b^{v}$ whenever it is a core allocation. The core for a game $v$ in $\mathbf{H}^{N}$ also contains only one element. This is due to the fact that each core allocation is contained in the imputation set $\operatorname{Imp}(v)$ which equals $\left\{(v(\{i\}))_{i \in N}\right\}$ for the games $v$ in $\mathbf{H}^{N}$.

Theorem 2.2 The cone $\mathrm{D}^{N}$ of games $v$ with Core $(v)=\left\{b^{v}\right\}$ is generated by the $2^{|N|}+|N|-1$ games $u^{\{i\}},-u^{\{i\}}$, with $i \in N$, and $-\mathbb{1}^{S}$, with $\emptyset \neq S \subset N$, $|S| \leq|N|-2$.

Proof: First, observe that

$$
\begin{aligned}
\sum_{j \in N} b_{j}^{v}-v(N) & =\sum_{j \in N \backslash\{i\}} b_{j}^{v}+v(N)-v(N \backslash\{i\})-v(N) \\
& =\sum_{j \in N \backslash\{i\}} b_{j}^{v}-v(N \backslash\{i\})
\end{aligned}
$$

holds for an arbitrary game $v$, i.e., the gaps $\sum_{j \in N \backslash\{i\}} b_{j}^{v}-v(N \backslash\{i\}), i \in$ $N$, corresponding to the $(|N|-1)$-person coalitions, equals the marginal gap. Using the core allocation $x=b^{v}$ for a game $v \in \mathbf{D}^{N}$ the weights corresponding to the games $-\mathbb{1}^{N \backslash\{i\}}, i \in N$, in the decomposition of (2.1) are zero according to $v(N)-\sum_{j \in N} b_{j}^{v}=0$ and (2.2). The theorem now follows immediately.

One similarly proves that 
Theorem 2.3 The cone $\mathbf{H}^{N}$ of games $v$ with Core $(v)=\left\{(v(\{i\}))_{i \in N}\right\}$ is generated by the $2^{|N|}+|N|-1$ games $u^{\{i\}},-u^{\{i\}}, i \in N$, and $-\mathbb{1}^{S}$, with $\emptyset \neq S \subset N,|S| \geq 2$.

The generating sets in the two latter theorems are minimal in the sense that no games can be deleted without losing the generating set property. Furthermore, they abey the property that each element in the generated cone has a unique representation as a non-negative weighted combination of the generating elements (in a representation it is assumed that either one of the weights of $u^{\{i\}}$ and $-u^{\{i\}}$ is 0 for each $\left.i \in N\right)$. This follows from the fact that the games in these classes have only one core allocation and from the cornment following (2.1).

\section{The 0,1-normalized games}

Theorem 2.1 is a natural generalization of a result in Spinetto [1974] concerning the class of 0,1 -normalized games with a non-empty core. For a game $v \in \mathbf{G}_{01}^{N}$ and core allocation $x$ we obviously have the following decomposition, similar to $(2.1)$,

$$
v=\sum_{i \in N} x_{i}\left(u^{\{i\}}-\mathbb{1}^{\{i\}}\right)+\sum_{S \subset N_{,}|S| \geq 2}\left(\sum_{j \in S} x_{j}-v(S)\right)\left(-\mathbf{1}^{S}\right) .
$$

Since $x \geq 0$ and $\sum_{j \in N} x_{j}=v(N)=1$ the following theorem is immediate.

Corollary 2.4 (Spinetto) The cone $\mathrm{C}_{01}^{N}$ equals the convex hull of the games $u^{\{i\}}-1^{\{i\}}, i \in N$, and $-1^{S}, S \subset N,|S| \geq 2$.

Again, by a similar reasoning as before one can show that none of the games in corollary 2.4 is redundant.

The 0-normalized games

From (2.3) we easily detect a generating set for the cone $\mathrm{C}_{0}^{N}$ of 0 normalized games with a non-empty core. It is stated in the next theorem together with results concerning the 0 -normalized games of the subclasses $\mathbf{D}^{N}$ and $\mathbf{H}^{N}$. Let us for the moment denote

$$
\begin{aligned}
& U=\left\{u^{\{i\}}: i \in N\right\} \cup\left\{-u^{\{i\}}: i \in N\right\} \text { and } \\
& W=\{-1: S \subset N, 2 \leq|S| \leq|N|-2\}) .
\end{aligned}
$$

We thus have

$$
\begin{aligned}
& \mathbf{A}^{N}=\operatorname{Cone}(U), \\
& \mathbf{C}^{N}=\operatorname{Cone}\left(U \cup W \cup\left\{-\mathbf{1}^{N \backslash\{i\}}: i \in N\right\} \cup\left\{-\mathbf{1}^{\{i\}}: i \in N\right\}\right), \\
& \mathbf{D}^{N}=\operatorname{Cone}\left(U \cup W \cup\left\{-\mathbf{1}^{\{i\}}: i \in N\right\}\right), \text { and } \\
& \mathbf{H}^{N}=\operatorname{Cone}\left(U \cup W \cup\left\{-\mathbf{1}^{N \backslash\{i\}}: i \in N\right\}\right) .
\end{aligned}
$$


Theorem 2.5 Let $|N| \geq 2$. Then

$$
\begin{aligned}
& \mathbf{C}_{0}^{N}=\operatorname{Cone}\left(\left\{u^{\{i\}}-\mathbb{1}^{\{i\}}: i \in N\right\} \cup W \cup\left\{-\mathbb{1}^{N \backslash\{\{i\}}: i \in N\right\}\right), \\
& \mathbf{D}_{0}^{N}=\operatorname{Cone}\left(\left\{u^{\{i\}}-\mathbf{1}^{\{i\}}: i \in N\right\} \cup W\right), \text { and } \\
& \mathbf{H}_{0}^{N}=\operatorname{Cone}\left(W \cup\left\{-\mathbf{1}^{N \backslash\{i\}}: i \in N\right\}\right) .
\end{aligned}
$$

Furthermore, the mentioned games in the generating sets are extreme in the corresponding classes.

Proof : The statements concerning the generating sets of $\mathbf{C}_{0}^{N}$ and $\mathbf{H}_{0}^{N}$ follow directly from (2.3). The statement with respect to $\mathbf{D}_{0}^{N}$ also follows from (2.3) using the core allocation $x=b^{v}$, the equality $\sum_{j \in N} b_{j}^{v}=v(N)$, and (2.2).

It remains to prove the extremality property of the games in the generating sets of $\mathbf{C}_{0}^{N}, \mathbf{D}_{0}^{N}$ and $\mathbf{H}_{0}^{N}$. The extremality of $-\mathbf{1}^{S}$ in $\mathbf{C}_{0}^{N}$, with $S \subset N,|S| \geq 2$, obviously follows from the fact that it is the only game in the generating set of $\mathbf{C}_{0}^{N}$ with a negative value for coalition $S$ and therefore it cannot be represented as a non-negative weighted sum of the other generating games.

Now suppose, for $j \in N$ arbitrary, that $u^{\{j\}}-1^{\{j\}}$ is a non-negative weighted sum of the other games in $G=\left\{u^{\{i\}}-\mathbb{1}^{\{i\}}: i \in N\right\} \cup\left\{-\mathbb{1}^{S}\right.$ : $S \subset N,|S| \geq 2\}$, say $u^{\{j\}}-\mathbf{1}^{\{j\}}=\sum_{w \in G} \alpha^{w} w$, with $\alpha^{u^{(j)}-\mathbf{1}^{(j)}}=0$. Then

$$
\begin{aligned}
\chi^{\{j\}=} & b^{u^{(j\}}-\mathbf{1}^{(j)}}=\sum_{w \in G} \alpha^{w} b^{w} \\
= & \sum_{i \in N} \alpha^{u^{\{i\}}-\mathbf{1}(i\}} b^{u^{(i)}-\mathbf{1}^{(i)}} \\
& -\sum_{S \subset N: 2 \leq|S| \leq|N|-2} \alpha^{-\mathbf{1}^{s}} b^{\mathbf{1}^{s}}-\sum_{i \in N} \alpha^{-\mathbf{1}^{N \backslash\{i\}} b^{\mathbf{1}^{N \backslash(i)}}} \\
= & \sum_{i \in N} \alpha^{u^{(i)}-\mathbf{1}(i)} \chi^{\{i\}}-\sum_{i \in N} \alpha^{-\mathbf{1}^{N \backslash(i)}}\left(-\chi^{\{i\}}\right)
\end{aligned}
$$

(observe that the marginal contribution allocation is considered here as a linear function on $\mathbf{G}^{N}$ with values in $I R^{N}$ ). This is only possible in case $\alpha^{-1^{N \backslash(i)}}=1$ for $i=j$ and 0 otherwise, and $\alpha^{u^{(i)}-N^{(i)}}=0$ for all $i \in N$. Thus, $u^{\{j\}}-\mathbb{1}^{\{j\}}$ is a positive weighted sum of the non-positive games $-\mathbb{1}^{S}$,

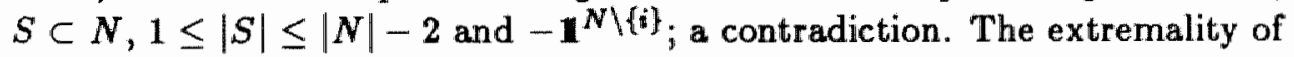
this game is now immediate.

The extremality of the games in the generating sets of $\mathbf{D}_{0}^{N}$ and $\mathbf{H}_{0}^{N}$ now follows from the extremality of these games in the larger cone $\mathrm{C}_{0}^{N}$. 
In case the player set $N$ consists of only two players then one simply checks that $\mathbf{C}_{0}^{N}=\operatorname{Cone}\left(\left\{\mathbf{1}^{N}\right\}\right)$ and $\mathbf{D}_{0}^{N}=\mathbf{H}_{0}^{N}=0$.

\subsubsection{A proof of the Bondareva-Shapley correspondence}

As an application of theorem 2.1 we will use this result in the proof of the characterization of a game with a non-empty core as a balanced game. This result has been shown independently by Bondareva [1963] and Shapley [1967]. In their proofs they basically use linear programming arguments, i.e., both showed that the characterization is an instance of the Duality Theorem (see also Charnes and Kortanek [1967]). Our proof is essentially based on theorem 2.1 and lemma 1.12 which is proved by the Separation Theorem.

Theorem 2.6 (Bondareva,Shapley) A game has a non-empty core if and only if it is balanced, i.e., $\mathbf{C}^{N}=\mathbf{B}^{N}$.

Proof: Let $D$ be the set of the generating games of $\mathbf{C}^{N}$, mentioned in theorem 2.1, and let $Y$ denote the set of balanced vectors, i.e.,

$$
\begin{gathered}
D=\left\{u^{\{i\}}: i \in N\right\} \cup\left\{-u^{\{i\}}: i \in N\right\} \cup\left\{-\mathbf{1}^{S}: \emptyset \neq S \subset N\right\} \text { and } \\
Y=\left\{\lambda \in I R^{2^{N} \backslash\{\emptyset\}:} \lambda_{N} \geq 0, \lambda_{S} \leq 0 \text { for } \emptyset \neq S \subset N,\right. \text { and } \\
\left.\sum_{S \ni i} \lambda_{S}=0 \text { for each } i \in N\right\} .
\end{gathered}
$$

Thus, $\mathbf{C}^{N}=\operatorname{Cone}(D)$ and $\mathbf{B}^{N}=\cap_{\lambda \in Y} \not(\lambda)$, where $\mathcal{H}(\lambda)$ is by definition the closed half-space of games $v$ for which $\lambda \cdot v=\sum_{\forall \neq S \subseteq N} \lambda_{S} v(S) \geq 0$. Using lemma 1.12 the cones $\mathbf{C}^{N}$ and $\mathrm{B}^{N}$ are equal if $Y$ consists of exactly those vectors $\lambda$ for which $D \subseteq N(\lambda)$ holds ( $Y$ is a cone by itself, implying $Y=\operatorname{Cone}(Y))$.

For a $\lambda \in Y$ the inclusion $D \subseteq \mathcal{H}(\lambda)$ follows from the observation on page 7, where we showed that a game with a non-empty core is balanced. We conclude that $Y \subseteq\left\{\lambda \in I R^{2^{N}} \backslash\{\theta\}: D \subseteq \mathcal{X}(\lambda)\right\}$.

Now suppose $D \subseteq \mathcal{K}(\lambda)$ for an arbitrary vector $\lambda$. Using $-\mathbf{1}^{S} \in D$ we obtain $0 \leq \lambda \cdot\left(-\mathbb{1}^{S}\right)=-\lambda_{S}$, i.e., $\lambda_{S} \leq 0$ for each $\neq S \subset N$. Using $u^{\{i\}} \in D$ we have $0 \leq \lambda \cdot u^{\{i\}}=\sum_{S \ni i} \lambda_{S}$, and from $-u^{\{i\}} \in D$ we obtain $0 \geq \sum_{S \ni i} \lambda_{S}$, i.e.,

$$
\sum_{S \ni i} \lambda_{S}=0 \text { for each } i \in N
$$

$\lambda_{N} \leq 0$ implies the non-positivity of the vector $\lambda$ and from (2.4) we now obtain that $\lambda$ must be the zero vector, in particular $\lambda_{N}=0$. We conclude 
that $\lambda$ is an element of $Y$; thus, $Y \supseteq\left\{\lambda \in I R^{2^{N}} \backslash\{(t): D \subseteq \mathcal{X}(\lambda)\}\right.$.

At the end of subsection 1.2.3 we showed that the class of balanced games is a subset of both classes $\mathbf{S}^{N}$ of semibalanced games and $\mathbf{Q}^{N}$ of quasi-balanced games. Using the equivalence of balancedness and the nonemptiness of the core this (again) shows that a game with a non-empty core is both semibalanced and quasi-balanced.

The set $Y$ of balanced vectors is a polyhedral cone, and the main result in Shapley [1967] is in fact the characterization of its extreme elements. Shapley showed that extreme balanced vectors are exactly those vectors $\lambda \neq 0$ which possess a minimal carrier $\left\{S \subset N: \lambda_{S}<0\right\}$; in other words a balanced vector $\lambda$ is extreme if and only if no other balanced vector $\mu$ exists with $\left\{S \subset N: \mu_{S}<0\right\} \subset\left\{S \subset N: \lambda_{S}<0\right\}$.

\subsubsection{The cone of non-negative games with a non-empty core}

Spinetto [1971] discusses the following result. Although its proof is based on the wrong assumption that in a simple game without veto-players there exist two disjoint winning coalitions, the theorem stays true.

Theorem 2.7 (Spinetto) The extreme elements of the compact and convex set of 0,1-normalized non-negative games with a non-empty core are the 0 normalized veto-controlled simple games.

We will provide an alternative proof based on the following generalization, which is the main result of this chapter.

Theorem 2.8 The cone $\mathbf{C}_{+}^{N}$ of non-negative games with a non-empty core is generated by the veto-controlled simple games. Furthermore, these games and their non-negative multiples are exactly the extreme directions of $\mathbf{C}_{+}^{N}$.

The proof is based on the properties of the set-valued function $F$ on the set $I R_{+}^{N}$ of non-negative allocations, which assigns to an allocation $x \geq 0$ the set $F(x)$ of games in $\mathbf{C}_{+}^{N}$ for which $x$ is a core allocation, i.e.,

$$
F(x)=\left\{v \in \mathbf{G}^{N}: v \geq 0, v \leq u^{x}, v(N)=\sum_{j \in N} x_{j}\right\}
$$

Thus, $F(x)$ consists precisely of those games which majorize $\left(\sum_{j \in N} x_{j}\right) 1^{N}$ and are majorized by the additive game $u^{x}$ corresponding to $x$. This implies 
that $F^{\prime}(x)$ equals $\left[\left(\sum_{j \in N} x_{j}\right) \mathbf{1}^{N}, u^{x}\right]$, the interval with top game $u^{x}$ and bottom game $\left(\sum_{j \in N} x_{j}\right) 1^{N}$. Notice that the dimension of $F(x)$ is $2^{|N|}-2$. In lemma 1.13 we characterized the extreme elements of these sets as precisely the ones with coordinates equal to the corresponding coordinates of either the top element or the bottom element of the interval.

In $a$ way $F$ is the inverse of the superadditive and homogeneous setvalued function Core: $F(x)=\left\{v \in \mathbf{G}^{N}: x \in \operatorname{Core}(v)\right\}$, with $x \in I R^{N}$. The obviously homogeneous function $F$ (of degree 1), however, obeys the stronger linearity property:

$$
F(x+y)=F(x)+F(y) \text { for all } x, y \in I R_{+}^{N} .
$$

This follows from the observation that the algebraic sum of each two nonempty intervals $\left[a^{0}, b^{0}\right]$ and $\left[a^{1}, b^{1}\right]$ in an arbitrary vector space equals $\left[a^{0}+\right.$ $\left.a^{1}, b^{0}+b^{1}\right]$ and, therefore,

$$
\begin{aligned}
F(x)+F(y) & =\llbracket\left(\sum_{j \in N} x_{j}\right) \mathbf{1}^{N}, u^{x} \rrbracket+\left[\left(\sum_{j \in N} y_{j}\right) \mathbf{1}^{N}, u^{y}\right] \\
& \left.=\llbracket \sum_{j \in N} x_{j} \mathbf{1}^{N}+\sum_{j \in N} y_{j} \mathbb{1}^{N}, u^{x}+u^{y}\right] \\
& =\left[\left(\sum_{j \in N}(x+y)_{j}\right) \mathbb{1}^{N}, u^{x+\nu}\right] \\
& =F(x+y)
\end{aligned}
$$

with $x, y \in I R_{+}^{N}$ arbitrary.

Proof: (of theorem 2.8) For a game $v \in \mathrm{C}_{+}^{N}$ with a core allocation $x \in I R_{+}^{N}$ we have

$$
v \in F(x)=F\left(\sum_{j \in N} x_{j} \chi^{\{j\}}\right)=\sum_{j \in N} x_{j} F\left(\chi^{\{j\}}\right) \text {. }
$$

Thus, $v$ is a non-negative weighted sum of games in the sets $F\left(\chi^{\{i\}}\right), i \in N$, and, therefore, $\mathbf{C}_{+}^{N}$ is generated by the extreme elements of these sets. According to lemma 1.13, the extreme games of $F\left(\chi^{\{i\}}\right)=\left[\mathbf{1}^{N}, u^{\{i\}}\right]$ are exactly all mixtures of the two simple games $\mathbb{1}^{N}$ and $u^{\{i\}}, i \in N$. We conclude that $\mathrm{C}_{+}^{N}$ is generated by a set of simple games. These simple games must be veto-controlled according to corollary 1.2 and extreme in $\mathbf{C}_{+}^{N}$ according to lemma 1.20 .

The above observation that the simple games are veto-controlled also follows from the fact that each mixture of the two games $\mathbb{1}^{N}$ and $u^{\{i\}}$ must be veto-controlled by player $i$. 
Theorem 2.8 is introduced in Derks [1987a], where it is proved with the help of a constructive method to decompose an arbitrary non-negative game, for which a core allocation is available, into a weighted sum of veto-controlled simple games. For further information concerning this method the reader is referred to subsection 2.2.4, where also an other procedure is discussed based on the implicit decomposition of a game with a non-empty core in the proof above.

Theorem 2.8 is also discussed in Curiel, Derks and Tijs [1989], where it is proved using Spinetto's characterization of the class of 0,1-normalized games with a non-empty core. Other applications of this theorem are discussed here.

In the last three sections results concerning games arising from a production model and a flow model are generalized with the help of theorem 2.8 .

\section{The proof of theorem 2.7}

We give a proof of Spinetto's 0,1-normalized version of theorem 2.8. Proof: (theorem 2.7) Let $v \in \mathbf{C}_{01}^{N} \cap \mathbf{G}_{+}^{N}$ and let $x \in I R^{N}$ be a core allocation. Thus, $x \geq 0$ and $\sum_{j \in N} x_{j}=1$. According to theorem 2.8 there is a positive weighted sum of veto-controlled simple games, say $\sum_{j \in J} \alpha^{j} w^{j}$, which equals v. From

$$
1=v(N)=\sum_{j \in J} \alpha^{j} w^{j}(N)=\sum_{j \in J} \alpha^{j}
$$

we conclude that $v$ is a convex combination of the games $w^{j}, j \in J$. Furthermore, from $v \geq \alpha^{j} w^{j}$ and $\alpha^{j}>0$ for each $j \in J$, and $v(\{i\})=0, i \in N$, we conclude that $w^{j}, j \in W$, are 0-normalized also. Therefore, the class $\mathbf{C}_{01}^{N} \cap \mathbf{G}_{+}^{N}$ is the convex hull of the 0-normalized veto-controlled simple games. The extremality of these games in the convex set $\mathbf{C}_{01}^{N} \cap \mathbf{G}_{+}^{N}$ follows from lemma 1.21 .

The class of games which are strategically equivalent to a game in the set $\mathbf{C}_{01}^{N} \cap \mathbf{G}_{+}^{N}=\operatorname{Conv}\left(\left\{w \in \mathbf{G}_{0}^{N}: w\right.\right.$ simple and veto-controlled $\left.\}\right)$ is equal to

$$
\text { Cone }\left(\left\{u^{\{i\}}: i \in N\right\}\right)+\operatorname{Cone}\left(\left\{w \in \mathbf{G}_{0}^{N}: w \text { simple and veto-controlled }\right\}\right. \text {. }
$$

It consists of precisely all those non-negative games with a non-empty core for which also

$$
v(S) \geq \sum_{j \in S} v(\{j\}) \text { for each non-empty coalition } S .
$$


The Bondareva-Shapley correspondence restricted to the non-negative games

Using theorem 2.8 and the Bondareva-Shapley correspondence as described in theorem 2.6 , the cone generated by the simple, veto-controlled games equals the set of games fulfilling the balancedness conditions and the non-negativity conditions; i.e., if

$$
\begin{aligned}
& D=\left\{w \in \mathbf{G}^{N}: w \text { simple, veto-controlled }\right\} \text { and } \\
& Y=\left\{\lambda \in I R^{2^{N} \backslash\{\emptyset\}}: \lambda_{N} \geq 0, \lambda_{S} \leq 0 \text { for } \emptyset \neq S \subset N\right. \text {, and } \\
& \left.\sum_{S \ni i} \lambda_{S}=0 \text { for each } i \in N\right\} \cup\left\{\mathbb{1}^{S}: \emptyset \neq S \subseteq N\right\}
\end{aligned}
$$

then Cone $(D)=\cap_{\lambda \in Y} \mathcal{H}(\lambda)$. Again this can be proved using theorem 2.8 and lemma 1.12, in the same way as is done in the proof of theorem 2.6. Since no game in the generating set $D$ can be left out, because of its extremality, the proof has to rely on the whole set $D$. A proof showing this dependency is therefore included.

Theorem 2.9 The class of the non-negative balanced games is generated by the veto-controlled simple games.

Proof: According to lemma 1.12 we have to prove the equality Cone $(Y)=$ $\left\{\lambda \in I^{2^{N} \backslash\{\phi\}}: D \subseteq \mathcal{H}(\lambda)\right\}$ with $D$ and $Y$ as described above. First, we obviously have $D \subseteq \mathcal{H}(\lambda)$ for all $\lambda \in Y$, implying $\operatorname{Cone}(Y) \subseteq\left\{\lambda \in I R^{2^{N} \backslash\{\phi\}}\right.$ : $D \subseteq \mathcal{H}(\lambda)\}$.

Now suppose $D \subseteq \mathcal{H}(\lambda)$ for an arbitrary $\lambda \in I R^{2^{N} \backslash\{\emptyset\}}$. We must have $\lambda_{N} \geq 0$ according to the fact that $\mathbb{1}^{N} \in D$. Now let $\mu \in I R^{2^{N}} \backslash\{\emptyset\}$ be the vector equal to $\lambda$ for coalitions $S$ with $\lambda_{S} \geq 0$ and $S \neq N$; the other entries of $\mu$ are assumed to be 0 . Then the vector $\lambda^{\prime}=\lambda-\mu$ fulfils $\lambda_{N}^{\prime}=\lambda_{N} \geq 0$ and $\lambda_{s}^{\prime} \leq 0$ for all $0 \neq S \subset N$. For the simple and veto-controlled game $w^{i}$ defined by $w^{i}(N)=1$ and $w^{i}(S)=1$ exactly for coalitions $S$ with $i \in S$ and $\lambda_{S}<0$ we have

$0 \leq w^{i} \cdot \lambda=w^{i} \cdot \lambda^{\prime}+w^{i} \cdot \mu=w^{i} \cdot \lambda^{\prime}=\sum_{S \ni i, \lambda_{S}<0} \lambda_{S}^{\prime}+\lambda_{N}^{\prime}=\sum_{S \ni i} \lambda_{S}^{\prime}=u^{\{i\}} \cdot \lambda^{\prime}$.

Now let $\lambda^{\prime \prime}=\lambda^{\prime}-\sum_{j \in N}\left(u^{\{j\}} \cdot \lambda^{\prime}\right) \mathbf{1}^{\{j\}}$. Then

$$
\sum_{S \ni i} \lambda_{S}^{\prime \prime}=\sum_{S \ni i} \lambda_{S}^{\prime}-u^{\{i\}} \cdot \lambda^{\prime}=0
$$

Of course, $\lambda^{\prime \prime}$ fulfils $\lambda_{N}^{\prime \prime} \geq 0$ and $\lambda_{S}^{\prime \prime} \leq 0$ for all $\emptyset \neq S \subset N$. Therefore, $\lambda^{\prime \prime}$ is a balanced vector. The vector $\lambda$ equals $\lambda^{\prime \prime}+\mu+\sum_{j \in N}\left(u^{\{j\}} \cdot \lambda^{\prime}\right) \mathbf{1}^{\{j\}}$ which 
equals $\lambda^{\prime \prime}$ incremented by a non-negative vector, i.e., $\lambda \in$ Cone $(Y)$. This proves the inclusion Cone $(Y) \supseteq\left\{\lambda \in I R^{2^{N} \backslash\{\theta\}}: D \subseteq \mathcal{K}(\lambda)\right\}$, and, therefore, equality holds.

\subsubsection{Constructive methods}

Let us consider (2.5) more closely. Let the games $v^{i} \in F\left(\chi^{(i)}\right), i \in N$, be such that we have $v=\sum_{j \in N} x_{j} v^{j}$. Notice that if $v$ is extreme in $F(x)$, i.e., $v \in \operatorname{Extr}(F(x))$, then the games $v^{i}, i \in N$, are also extreme in $F\left(\chi^{(i)}\right)$. Extremality of $v$ in $F(x)=\left[\left(\sum_{j \in N} x_{j}\right) \mathbf{1}^{N}, u^{x}\right]$ implies that there is a subset $\Omega \subseteq 2^{N} \backslash\{\emptyset\}$ of non-empty coalitions such that $v=\operatorname{mix} n\left(\sum_{j \in N} x_{j} \mathbf{1}^{N}, u^{x}\right)=$ $\operatorname{mix}_{n}\left(u^{x}\right)$, assuming that $N$ is contained in $\Omega$. Then $v^{i}, i \in N$, are the games $\operatorname{mix}_{\Omega}\left(u^{\{i\}}\right)$, i.e., simple and veto-controlled. Now if $v$ is a convex combination of extreme games of $F(x)$ then each game $v^{i}, i \in N$, may be chosen as the game which is the same convex combination of the corresponding extreme games of $F\left(\chi^{\{i\}}\right)$. Therefore, in order to represent a non-negative game $v$ with a core allocation $x$ as a non-negative weighted sum of simple, veto controlled games, we have to represent $v$ as a convex combination of extreme games of $F(x)$. In (1.19) a decomposition is given representing an element of an interval as a convex combination of mixtures of the top element and the bottom element of the interval, which are the extreme elements. Since $F(x)$ is an interval we may apply (1.19) on the game $v$.

The computational aspects of the procedure which results from the above observations are strongly dependent on the choice of the core allocation $x$ of the game $v$ which is to be represented as a non-negative weighted sum of simple veto-controlled games. The procedure provides a desired representation using the lowest number of distinct simple veto-controlled games whenever an allocation $x \in \operatorname{Core}(v)$ is chosen such that the set $\left\{\emptyset \neq S \subseteq N: u^{x}(S)=v(S)\right\}$ is as large as possible.

The slice procedure

An other procedure is introduced in Derks [1987a]. The heart of this procedure is the computation of a 'slice' $\alpha w$ of a given game $v$ and core allocation $x$, such that

- $w$ is simple and veto-controlled, say by $i \in N$;

- the weight $\alpha$ is positive with $v-\alpha w \in \mathbf{G}_{+}^{N}$, and

- $x-\alpha \chi^{\{i\}} \in \operatorname{Core}(v-\alpha w)$. 




Figure 2.1: The slice $\alpha w$

Lemma 2.10 Let $v \in \mathbf{C}_{+}^{N} \backslash\{0\}$ and $x \in \operatorname{Core}(v)$. For a player $i$ with $x_{i}>0$ let the game $w$ be defined by $w(S)=1$ if $v(S)>0, i \in S$, and $w(S)=0$ otherwise. Then $w$ is simple and veto-controlled.

Let $\alpha$ denote the minimum of $x_{i}$ and $\min (\{v(S): S \subseteq N$ with $w(S)=1\})$. Then $x-\alpha \chi^{\{i\}} \in \operatorname{Core}(v-\alpha w)$.

Proof : The allocation $x$ cannot be zero; thus there is a player $i$ with positive entry $x_{i}$. The $\{0,1\}$-valued game $w$, as defined in the lemma, is simple since the assertion $w(N)=1$ follows from $v(N) \geq x_{i}>0$. Of course, $i$ is a veto-player of $w$.

Let us consider the second assertion.

For all $\emptyset \neq S \subset N$, not containing player $i$ :

$$
(v-\alpha w)(S)=v(S) \leq \sum_{j \in S} x_{j}=\sum_{j \in S}\left(x-\alpha \chi^{\{i\}}\right)_{j}
$$

for all $S \subseteq N, i \in S$, with $v(S)>0$ :

$$
(v-\alpha w)(S)=v(S)-\alpha \leq \sum_{j \in S} x_{j}-\alpha=\sum_{j \in S}\left(x-\alpha \chi^{\{i\}}\right)_{j} ;
$$

and for all $S \subseteq N$, $i \in S$, with $v(S)=0$ :

$$
(v-\alpha w)(S)=v(S)=0 \leq x_{i}-\alpha \leq \sum_{j \in S}\left(x-\alpha \chi^{\{i\}}\right)_{j}
$$

Furthermore, $x-\alpha \chi^{\{i\}}$ is an efficient allocation since we have

$$
\sum_{j \in N}\left(x-\alpha \chi^{[i\}}\right)_{j}=v(N)-\alpha=(v-\alpha w)(N)
$$


We conclude that $x-\alpha \chi^{\{i\}}$ is a core allocation of $v-\alpha w$.

Observe that combining the above lemma with theorem 1.15 again yields the result that $\mathbf{C}_{+}^{N}$ is generated by the simple veto-controlled games.

The scalar $\alpha$ in the above lemma is chosen as large as possible: for a scalar $\alpha^{\prime}>\alpha$ we have

- either $\alpha^{\prime}>x_{i}$, implying $x-\alpha \chi^{\{i\}} \notin I R_{+}^{N}$

- or $\alpha^{\prime}>v(S)$ for some coalition $S$ with $w(S)=1$, implying $v-\alpha w \notin$ $\mathrm{G}_{+}^{N}$.

If $N(y)$ denotes the number of non-zero entries in an arbitrary vector $y$ we thus have either $\mathcal{N}(x)>\mathcal{N}\left(x-\alpha \chi^{\{i\}}\right)$ or $\mathcal{N}(v)>\mathcal{N}(v-\alpha w)$. This implies

$$
2^{|N|}-1+|N| \geq \mathcal{N}(v)+\mathcal{N}(x)>\mathcal{N}(v-\alpha w)+\mathcal{N}\left(x-\alpha \chi^{\{i\}}\right) \geq 0 .
$$

This argument is essential in the proof that the following procedure will terminate after a finite number of loops.

\section{Procedure 2.11}

Step 1 Let $k=0, v^{0}=v$ and $x^{0}=x$.

Step 2 If $x^{k}=0$ then STOP.

Step 3 Choose $i \in N$ such that $x_{i}>0$.

Let $w^{k}$ be the game defined by $w^{k}(S)= \begin{cases}1 & \text { if } S \ni i \text { and } v^{k}(S)>0, \\ 0 & \text { otherwise, }\end{cases}$ and $\alpha^{k}=\min \left(\left\{x_{i}^{k}\right\} \cup\left\{v(S): S \subseteq N\right.\right.$ with $\left.\left.w^{k}(S)=1\right\}\right)$.

Let $v^{k+1}=v^{k}-\alpha^{k} w^{k}$ and $x^{k+1}=x^{k}-\alpha^{k} \chi^{\{i\}}$.

Step 4 Increase $k$ by 1 ; GOTO step 2.

Theorem 2.12 For a game $v \in \mathbf{C}_{+}^{N}$ and allocation $x \in$ Core $(v)$ procedure 2.11 yields a finite number of positive weighted simple veto-controlled games $\alpha^{k} w^{k}, 1 \leq k \leq K$, such that $v=\sum_{k=1}^{K} \alpha^{k} w^{k}$.

Proof: By construction all computed games and allocations are nonnegative whenever we start with a non-negative game $v$ and allocation $x \geq 0$. Now according to lemma 2.10 we have $x^{k+1} \in \operatorname{Core}\left(v^{k+1}\right)$ provided that 
$x^{k} \in \operatorname{Core}\left(v^{k}\right)$. The assumption $x^{0} \in \operatorname{Core}\left(v^{0}\right)$, thus, implies $x^{k} \in \operatorname{Core}\left(v^{k}\right)$ for each $k$. According to (2.7) the sequence of non-negative numbers

$$
\mathcal{N}\left(v^{0}\right)+\mathcal{N}\left(x^{0}\right), \mathcal{N}\left(v^{1}\right)+\mathcal{N}\left(x^{1}\right), \ldots
$$

is strictly decreasing. It therefore terminates at some point, say at $k=K+1$, implying $x^{K+1}=0$. Since $x^{K+1} \in \operatorname{Core}\left(v^{K+1}\right)$ the game $v^{K+1}$ is the alllosing game. Therefore,

$$
\begin{aligned}
v & =v^{0}=v^{1}+\alpha^{1} w^{1}=v^{2}+\sum_{k=1}^{2} \alpha^{k} w^{k}=\ldots \\
& =v^{K+1}+\sum_{k=1}^{K} \alpha^{k} w^{k} \\
& =\sum_{k=1}^{K} \alpha^{k} w^{k}
\end{aligned}
$$

Let $i^{k}$ denote the player who is chosen in step 3 in the $k^{\text {th }}$ loop. We must have

$$
x=x^{0}=\sum_{k=0}^{K} \alpha^{k} \chi^{\left\{i^{k}\right\}} .
$$

Now if player $i \neq i^{k}$ is an other veto-player of $w^{k}$ then $x-\alpha^{k} \chi^{\left\{i^{k}\right\}}+\alpha^{k} \chi^{\left\{i^{k}\right\}}$ denotes also a core allocation. The procedure may thus be of help in locating (nearby) core allocations of $x$.

Suppose the procedure is started with an arbitrary efficient allocation then the procedure still terminates after a finite number of loops. Again, the games $w^{k}$ are simple and veto-controlled, and the weights $\alpha^{k}$ are positive by construction, for each $k$ the procedure is in step 3 . Furthermore, (2.8) and also (2.9) are still valid. The last computed game $v^{K+1}$, however, may not be equal to the all-losing game. Notice that at each loop $k$ the allocation $x^{k+1}$ is efficient for the game $v^{k+1}$ whenever $x^{k}$ is efficient for $v^{k}$. Therefore, we must have $v^{K+1}(N)=0$. If the game $v^{K+1}$ is equal to the all-losing game then we have $v=\sum_{k=1}^{K} \alpha^{k} w^{k}$, yielding

$$
x=\sum_{k=1}^{K} \alpha^{k} \chi^{i^{k}} \in \sum_{k=1}^{K} \alpha^{k} \operatorname{Core}\left(w^{k}\right) \subseteq \operatorname{Core}\left(\sum_{k=1}^{K} \alpha^{k} w^{k}\right)=\operatorname{Core}(v)
$$

Therefore, 
Corollary 2.13 If procedure 2.11 is started with a non-negative game $v$ and a non-negative efficient allocation $x$ then it terminates after a finite number $K$ of loops. We have $x \in \operatorname{Core}(v)$ if and only if $v^{K+1}=0$.

Procedure 2.11 can thus be used also for testing whether an allocation is contained in the core of a game. We emphasize, however, that checking the core inequalities $v(S) \leq \sum_{j \in S} x_{j}, \emptyset \leq \subseteq N$, is at least as efficient.

\section{An example}

The following example is considered in Derks [1987a]. It demonstrates the decomposition of a 4-person game using procedure 2.11. This is done in the following table. The worths of the non-supplemented coalitions in the table are 0 for the considered games. Observe that in step 3 the choice of a player with positive coordinate value in $x$ may be arbitrary; however, here we choose a player with the smallest index.

\begin{tabular}{l|ccccccc|c} 
& $\{1,2\}$ & $\{1,3\}$ & $\{1,4\}$ & $\{2,3\}$ & $\{2,4\}$ & $\{3,4\}$ & $N$ & \\
\hline Step 1: & $k=0$, & & & & & \\
$v^{0}$ & 1 & 1 & 1 & 1 & 1 & 2 & 3 & $x^{0}=(1,0,1,1)$ \\
Step 2: & $x^{0} \neq 0$,
\end{tabular}

Step 4: $k=1$.

Step 2: $x^{\mathbb{1}} \neq 0$.

Step 3: Let $i$ be player 3;

\begin{tabular}{l|lllllll|l}
$w^{1}$ & 0 & 0 & 0 & 1 & 0 & 1 & 1 & $\alpha^{1}=1$ \\
$v^{2}$ & 0 & 0 & 0 & 0 & 1 & 1 & 1 & $x^{2}=(0,0,0,1)$
\end{tabular}

Step 4: $k=2$.

Step 2: $x^{2} \neq 0$.

Step 3: Let $i$ be player 4;
\begin{tabular}{l|l}
$w^{2}$ & 0 \\
$v^{3}$ & 0
\end{tabular}
$0 \quad 0$
0
$\begin{array}{ll}1 & 1 \\ 0 & 0\end{array}$
\begin{tabular}{l|l}
1 & $\alpha^{2}=1$ \\
0 & $x^{3}=(0,0,0,0)$
\end{tabular}

Step 4: $k=3$.

Step 2: $x^{3}=0$; STOP. 


\subsubsection{Population monotonic allocation schemes}

Let us consider here the class of games $v$ for which there exist a collection of allocations $\left(x^{\mathcal{S}}\right)_{S \subseteq N, S \neq \emptyset}$ with the three properties

$$
\begin{array}{ll}
\text { - } & x_{j}^{S}=0 \text { for each coalition } S \text { and player } j \notin S \\
\text { - } & \sum_{j \in S} x_{j}^{S}=v(S) \text { for each coalition } S, \\
\text { - } & x_{i}^{S} \leq x_{i}^{T} \text { for each } i \in N \text { and } S, T \subseteq N, i \in S \subseteq T .
\end{array}
$$

A collection with these properties is called a population monotonic allocation scheme (PMA-scheme for short). It easily follows that the allocations $x^{S}$, $S \subseteq N$, in a PMA-scheme are non-negative, implying that the game $v$ itself must be non-negative.

A PMA-scheme can be considered as a collection of core allocations for the subgames. This follows from the observation that for a game $v$ with PMA-scheme $\left(x^{\mathcal{S}}\right)_{\emptyset \neq S \subseteq N}$ we have, for each coalition $T \subseteq N$,

$$
v_{\mid T}(S)=v(S)=\sum_{j \in S} x_{j}^{S} \leq \sum_{j \in S} x_{j}^{T} \text { for each coalition } S \subseteq T
$$

the efficiency of the allocation $x^{T}$ in the game $v_{\mid T}$ follows from (2.10) and (2.11). Therefore, $x^{T}$ is a core allocation of $v_{\mid T}$.

For a comprehensive discussion of this subject the reader is referred to Moulin [1989] and Sprumont [1990]. We will discuss here the results in the latter article, where the class of games with a PMA-scheme, denoted by $\mathbf{P M}^{N}$, is characterized, both by constructing a generating set and by the construction of a set of normal vectors, which intersection of the corresponding closed half-spaces yields the class $\mathbf{P M}^{N}$.

\section{A generating set}

The class $\mathbf{P M}^{N}$ is generated by its simple games:

Theorem 2.14 (Sprumont) The class $\mathrm{PM}^{N}$ is generated by the set of monotonic simple games with veto-control. Furthermore, the latter games and their positive multiples are the extreme directions of $\mathrm{PM}^{N}$.

Proof: Let $v \in \mathbf{P M}^{N} \backslash\{0\}$ and let $\left(x^{S}\right)_{0 \neq S \subseteq N}$ be a PMA-scheme of $v$. Consider the following $|N|$ games $w^{i}, i \in N$, defined by $w^{i}(S)=x_{i}^{S}, S \subseteq N$. The monotonicity of these games follows from the monotonicity property 
(2.12). Using the second property (2.11) we obtain

$$
v=\sum_{j \in N} w^{*}
$$

According to theorem 1.22 we may assume that each of the games $w^{i}, i \in N$, is a positive weighted sum, say $w^{i}=\sum_{r \in R^{i}} \alpha^{r i} w^{r i}$, of simple monotonic games. Then $w^{i} \geq w^{r i}$ for each $i \in N$ and $r \in R^{i}$. Now using the fact that $w^{i}(S)=x_{i}^{S}=0$ for $S \subset N$ and $i \in N \backslash S$, we conclude that $w^{r i}(S)=0$ for each coalition $S$ not containing player $i$. Thus, all involved simple games are veto-controlled. Together with (2.13) this implies that $v$ is a positive weighted sum of monotonic simple veto-controlled games.

The latter games are contained in $\mathbf{P M}^{N}$ since for a veto-player $i$ of a monotonic simple game $w$ the collection $\left(w(S) \chi^{\{i\}}\right)_{\phi \neq S \subseteq N}$ is a PMA-scheme of $w$. Furthermore, these games are extreme since they are extreme in the larger class of non-negative games with a non-empty core.

In accordance with the Bondareva-Shapley correspondence the games with a non-empty core for each subgame are exactly the totally balanced games. Therefore, The class $\mathbf{P M}^{N}$ is a subset of $\mathbf{T B}_{+}^{N}$. The monotonic vetocontrolled simple games are, thus, totally balanced simple games. These are exactly the totally balanced simple games, since a non-negative totally balanced game is monotonic.

In section 4.3.1 we shall see that the class $\mathbf{T B}_{+}^{N}$ of non-negative totally balanced games actually unequals the cone generated by its simple games. This shows that $\mathbf{P M}^{N}$ is a non-trivial subset of $\mathbf{T B}_{+}^{N}$.

\section{The inequality conditions}

Sprumont [1990] also presents the inequality conditions for a game to be contained in $\mathbf{P M}^{N}$ "For the introduction of these conditions consider a vector $\lambda \in I R^{2^{N}} \backslash\{\emptyset\}$ to be subbalanced if $\sum_{S \in B} \lambda_{S} \geq 0$ holds for all monotonic vetocontrolled families $B \subseteq 2^{N}$ of coalitions. Here a family $B$ of coalitions is said to be monotonic if $S \in B$ implies $T \in B$ for each coalition $T$ containing $S$ as a subset; $B$ is called veto-controlled if there is an $i \in N$ such that $S \in B$ implies $i \in S$. Let $y$ denote the set of subbalanced vectors. Sprumont proved that a 0 -normalized game $v$ has a PMA-scheme if and only if it fulfils

$$
\sum_{S \subseteq N} \lambda_{S} v(S) \geq 0 \text { for all } \lambda \in y \text {. }
$$

In fact we have the slightly more general result: 
Theorem 2.15 Let $v$ be a non-negative game. Then $v$ has a PMA-scheme if and only if it fulfils (2.11).

Proof: For a monotonic collection $\emptyset \neq B \subseteq 2^{N} \backslash\{\emptyset\}$, for which there is a player $i \in N$ such that $i \in S$ for all $S \in B$, the corresponding $\{0,1\}$-valued game $w$, with $w(S)=1$ if and only if $S \in B$, is monotonic, simple and veto-controlled. The subbalancedness property of a vector $\lambda$ is, therefore, equivalent to

$\lambda \cdot w \geq 0$ for all simple, monotonic and veto-controlled games $w$.

Let $D$ denote the set of simple, monotonic and veto-controlled games. Then we have $\{\lambda: D \subset \mathcal{H}(\lambda)\}=y$. According to lemma 1.12 we, therefore, conclude that $\mathbf{P M}^{N}$, which equals Cone $(D)$ according to theorem 2.14, coincides with $\cap_{\lambda \in Y} \mathbb{K}(\lambda)$, which consists exactly of those games for which (2.14) holds.

\subsubsection{The airport cost savings games}

As mentioned in section 1.2.6, the savings games associated to the airport cost functions have a non-empty core. Recall that an airport cost savings game $w^{x}$ for an allocation $x \in I R^{N}$ is defined by

$$
w^{x}(S)=\sum_{j \in S} x_{j}-\max _{S \ni i} x_{i}
$$

The cone generated by the airport cost savings games associated to the nonnegative allocations will play a significant role in the decomposition of the class of the so-called semiconvex games, treated in subsection 3.4.1.

The class of airport cost savings games, however, is not a convex set as is illustrated by the following example. Consider the player set $N=\{1,2,3\}$ (the example can easily be extended to arbitrarily large $N$ ) and the savings games $w^{x}$ and $w^{y}$ associated to the airport cost functions with the cost allocations $x=(0,1,2)$ and $y=(2,1,0)$. In fact, the games $w^{x}, w^{y}$ and $w=w^{x}+w^{y}$ are as follows.

\begin{tabular}{l|cccc}
$S$ & $\{1,2\}$ & $\{1,3\}$ & $\{2,3\}$ & $N$ \\
\hline$w^{x}(S)$ & 0 & 0 & 1 & 1 \\
$w^{v}(S)$ & 1 & 0 & 0 & 1 \\
$w(S)$ & 1 & 0 & 1 & 2
\end{tabular}


(the not included coalitions have worth 0 for each of the three games). Suppose $w$ equals $w^{z}$ for a certain allocation $z \in I R^{3}$. Then we have, for each two-person coalition $S$,

$$
w(S)=w^{x}(S)=\sum_{j \in S} z_{j}-\max _{j \in S} z_{j}=\min _{j \in S} z_{j} .
$$

From this and $w(\{1,3\})=0$, we first derive that $z_{1}=0$ or $z_{3}=0$, and consequently, $w(\{1,2\}) \leq 0$ or $w(\{2,3\}) \leq 0$; a contradiction. We conclude that $w$ cannot be an airport cost savings game.

Now the idea is to embed the class of the airport games in a convex cone generated by a finite number of appropriately defined airport cost savings games. For the sake of notation, the airport cost savings game associated with the allocation $\chi^{S}$ is shortly denoted by $w^{S}$. Thus,

$$
w^{S}(T)=\sum_{j \in T} \chi_{j}^{S}-\max _{i \in T} \chi_{i}^{S}=\left\{\begin{array}{cl}
|S \cap T|-1 & \text { if } S \cap T \neq \emptyset \\
0 & \text { otherwise. }
\end{array}\right.
$$

We note that for coalitions $S$, with $|S| \geq 2$, the marginal contribution allocation $b^{w^{S}}$ of the game $w^{S}$ equals the original allocation $\chi^{S}$ and in case $|S| \leq 1$ then $w^{S}$ equals the all-losing game, and, thus, $b^{w^{S}}=0$.

The next theorem states that a airport cost savings game associated with a non-negative allocation is a non-negative linear combination of the games $w^{S}, S \subseteq N$.

Theorem 2.16 The cone $\mathrm{AC}^{N}=\operatorname{Cone}\left(\left\{w^{S}: S \subseteq N\right\}\right)$ is the smallest cone containing the airport cost savings games $w^{x}, x \in I_{+}^{n}$.

Proof: Let $x \in I R_{+}^{N}$. Let the players be numbered such that $N=$ $\{1,2, \ldots, n\}$ and $0=x_{0} \leq x_{1} \leq x_{2} \leq \ldots \leq x_{n}$, with $|N|=n$. For $j \in N$, define the set $S_{j}=\{j, j+1, \ldots, n\}$ of players with index $j$ or a larger index. It is evident that the vector equality $x=\sum_{j=1}^{n}\left(x_{j}-x_{j-1}\right) \chi^{S_{j}}$ holds. We claim that

$$
w^{x}=\sum_{j=1}^{n}\left(x_{j}-x_{j-1}\right) w^{s_{j}}
$$

To prove (2.16) let $T \subseteq N, T \neq \emptyset$. Further, let $m \in T$ be the player in $T$ with the largest index. We have $S_{j} \cap T=0$ if and only if $m<j$. It follows that

$$
\sum_{j=1}^{n}\left(x_{j}-x_{j-1}\right) w^{S_{j}}(T)=\sum_{j=1}^{m}\left(x_{j}-x_{j-1}\right)\left(\left|S_{j} \cap T\right|-1\right)
$$




$$
\begin{aligned}
& =\sum_{j=1}^{m}\left(x_{j}-x_{j-1}\right) \sum_{k=j}^{m} \chi_{k}^{T}-\sum_{j=1}^{m}\left(x_{j}-x_{j-1}\right) \\
& =\sum_{j=1}^{m} \sum_{k=j}^{m}\left(x_{j}-x_{j-1}\right) \chi_{k}^{T}-\left(x_{m}-x_{0}\right) \\
& =\sum_{k=1}^{m} \sum_{j=1}^{k}\left(x_{j}-x_{j-1}\right) \chi_{k}^{T}-x_{m} \\
& =\sum_{k=1}^{m} x_{k} \chi_{k}^{T}-x_{m} \\
& =\sum_{k \in T} x_{k}-\max _{k \in T} x_{k} \\
& =w^{x}(T) .
\end{aligned}
$$

We conclude that (2.16) holds. This proves that each airport cost savings game associated with non-negative allocations is contained in $\mathbf{A C}^{N}$. Now $\mathrm{AC}^{N}$ is the smallest cone with this property since it is generated by airport cost savings games associated with $\chi^{S}, S \subseteq N$.

Actually the games $w^{S}, S \subseteq N$, are extreme in $\mathbf{A C}^{N}$. This will also be derived in subsection 3.4 .1 in a more general setting.

The decomposition in (2.16) has a nice application in the determination and interpretation of the Shapley value allocation of the airport cost savings game $w^{x}$. The linearity property of $\phi()$ implies that $\phi\left(w^{x}\right)$ equals the sum $\sum_{j=1}^{n}\left(x_{j}-x_{j-1}\right) \phi\left(w^{S_{j}}\right)$. The Shapley value of an airport cost savings game $w^{S}$ equals

$$
\phi\left(w^{s}\right)=\frac{|S|-1}{|S|} \chi^{s}, \quad \emptyset \neq S \subseteq N,
$$

i.e., the total $|S|-1$ of the savings of the grand coalition is equally distributed among the players in $S$. Now (2.16) implies that the total $w^{x}(N)=$ $\sum_{j \in N} x_{j}-\max _{i \in N} x_{i}$ of savings is built up of the incremental savings $\left(x_{j}-\right.$ $\left.x_{j-1}\right)\left(\left|S_{j}\right|-1\right)$, which are contributed by the players in $S_{j}, j=1, \ldots, n$. The equality $\phi\left(w^{x}\right)=\sum_{j=1}^{n}\left(x_{j}-x_{j-1}\right) \phi\left(w^{S_{j}}\right)$ suggests that each of these incremental savings is distributed equally among the players who made these savings possible.

These observations correspond in fact to the following simple cost allocation rule for an airport cost function as treated in Littlechild and Owen [1973]. Divide the lowest cost $x_{1}$ equally among all players. Divide the incremental cost $x_{2}-x_{1}$ (above the lowest cost $x_{1}$ ) equally among all players 
except player 1 . Continue this procedure thus until the incremental cost $x_{n}-x_{n-1}$ is allocated to player $n$. The Shapley value allocation of the corresponding airport cost savingsgame is determined by the difference of the cost allocation $x$ itself and the cost allocation resulting from the above mentioned simple rule.

\subsection{Linear programming games}

Consider the following model in which production of $k$ different products is possible with the use of $m$ available resources. Let $a_{i j}$ denote the amount of resource $i$ needed in the production of one unit of product $j$. It is allowed that some of the entries $a_{i j}$ may be negative, i.e., production may enlarge the amount of some of the available resources.

Let $A$ be the $(m \times k)$-matrix $\left(a_{i j}\right)_{1 \leq i \leq m, 1 \leq j \leq k}$. For $x \in I R_{+}^{k}$ the product $A x=\left(\sum_{j=1}^{k} a_{i j} x_{j}\right)_{1 \leq i \leq m}$ denotes the amount needed of each of the resources in order to produce the amount $x_{j}, 1 \leq j \leq k$, of each product $j$. For a resource constraint vector $b \in I R_{+}^{m}$ the feasible region $\left\{x \in I R_{+}^{k}: \quad A x \leq b\right\}$ consists of those production bundles which actually can be produced with the amounts of resources given by $b$. In this section we assume that $A$ has the property that the set $\left\{x \in I R_{+}^{k}: A x \leq b\right\}$ is bounded for each $b \in I R_{+}^{m}$. The following lemma characterizes those matrices.

Lemma 2.17 The set $\left\{x \in I R_{+}^{k}: A x \leq b\right\}$ is bounded for each $b \in I R_{+}^{m}$ if and only if there exists a vector $y \in I R_{+}^{m}$ such that $y^{t} A>0^{t}$.

\section{Proof:}

('only if'-part) Boundedness of $\left\{x \in I R_{+}^{k}: A x \leq b\right\}$ implies that $\max \{c \cdot x: x \geq 0, A x \leq b\}$ exists for arbitrary $c \in I R^{k}$ and this maximum equals $\min \left\{y \cdot b: y \geq 0, y^{t} A \geq c^{t}\right\}$ according to the Duality Theorem. In particular, this is true for a positive vector $c$, which implies that there is a vector $y \in I R_{+}^{m}$ such that $y^{t} A>0^{t}$.

('if'-part) Now let $y \in I R_{+}^{m}$ be such that $y^{t} A>0^{t}$. Then for each $b \in I R_{+}^{m}$ and $x \in I R_{+}^{k}$ with $A x \leq b$ we have

$$
y \cdot b \geq y \cdot(A x)=\left(y^{t} A\right)^{t} \cdot x \geq\left(y^{t} A\right)_{j} x_{j}, \quad 1 \leq j \leq k .
$$

Thus, $\left\{x \in I R_{+}^{k}: A x \leq b\right\}$ is contained in the interval $\left[0,\left(y \cdot b /(y A)_{j}\right)_{1 \leq j \leq k}\right]$, and this proves the 'if'-part.

The vector $y \in I R_{+}^{m}$ in the above lemma and proof may be regarded as a (shadow) price vector for the resources. In this context the amount $\left(y^{t} A\right)_{j}$ 
is the cost price of producing 1 unit of product $j$, assuming that the only costs in the production are induced by the resources. In accordance with the above lemma we therefore assume in this section that there exists a vector $y \in I R_{+}^{m}$ with $y^{t} A>0^{t}$, i.e., there exists a price vector on the resources such that the production of each product involves some positive costs. If $A$ is a non-negative matrix with non-zero column vectors then each positive vector $y$ will do.

Now if furthermore a non-negative price vector $c \in I R_{+}^{k}$ is given for the products then the maximal profit that can be made is given by

$$
\max \left\{c \cdot x: x \in I R_{+}^{k}, A x \leq b\right\} .
$$

Consider the situation that for a group $N$ of players it is known how much of the resources can be released by each subgroup of $N$; thus a collection $\left(b^{S}\right)_{s \subseteq N}$ of non-negative constraint vectors is given, where $b^{b}$ is assumed to be the zero constraint vector. The tuple $\left\langle A,\left(b^{S}\right)_{S \subseteq N}\right\rangle$ is called a controlled linear programming model.

This model gives rise to the following game $v^{c}$ with player set $N$, and defined by

$$
v^{c}(S)=\max \left\{c \cdot x: x \in \mathbb{R}_{+}^{k}, A x \leq b^{S}\right\}, \quad S \subseteq N,
$$

with $c \in I R_{+}^{k}$ a price vector. This game is called a linear programming game.

\subsubsection{Linear production games}

Let us first list some approaches and results with respect to linear programming garnes. Proofs are omitted since most of the results here follow from a general result which will be discussed and proved in the subsection following this one.

\section{Owens' approach}

Study of linear programming games started in Owen [1975], where situations are considered with $A$ a non-negative matrix and

$$
b^{S}=\sum_{j \in S} b^{\{j\}}, \quad 0 \neq S \subseteq N
$$

Games arising from these models are called linear production games. Then Theorem 2.18 (Owen) A linear production game is totally balanced. 
A collection $\left(b^{S}\right)_{S \subseteq N}$ of $m$-dimensional vectors can be seen as $m$ different non-negative games, for each coordinate one game. We refer to these games as the constraint games (in subsection 2.2 .5 we encountered the situation that a collection of allocations $\left(x^{S}\right)_{S \subseteq N}$ gave rise to $|N|$ games in a similar way). A collection $\left(b^{S}\right)_{S \subseteq N}$ is, therefore, called (totally) balanced if the $m$ corresponding constraint games are (totally) balanced. Observe that property (2.18) is equivalent to the additivity of the $m$ constraint games.

\section{The approach of Granot}

Granot [1986] considered linear pogramming models with non-negative matrix $A$ and totally balanced collections of constraint vectors. The games arising from these models are called generalized linear production games. These games are indeed generalizations of the linear production games since the additivity of the constraint games, implied by (2.18), yields the necessary total balancedness property.

Theorem 2.19 (Granot) A generalized linear production game is totally balanced.

\section{Committee control}

In Curiel, Derks and Tijs [1989] and Curiel [1988] simple veto-controlled games are used to describe the control of the involved constraint vectors. This type of control is inspired by the observation that in Owen's controlled linear programming model the control of the $|N|$ constraint vectors $b^{\{i\}}$, $i \in N$, is in fact modelled by the dictator games. Simple games are especially suitable for modelling (committee) control since the worth of a coalition in such a game denotes whether the coalition controls (value is 1) or does not control (value is 0 ) the resource vector in question. See also Dubey and Shapley [1984] where this kind of control is applied for the first time.

Games are referred to as veto-controlled linear production games if they arise from controlled linear programming models with non-negative matrix $A$ and constraint vectors

$$
b^{S}=\sum_{r \in R} w^{r}(S) b^{r}, \quad \emptyset \neq S \subseteq N,
$$

where $b^{r}, r \in R$, is a finite collection of resource constraint vectors and $w^{r}$, $r \in R$, are veto-controlled simple games. Then

Theorem 2.20 (Curiel, Derks, Tijs) A veto-controlled linear production game is balanced. 
According to the following lemma the application of veto-controlled simple games is equivalent to permitting balanced collections $\left(b^{S}\right)_{S \subseteq N}$ of constraint vectors.

Lemma 2.21 The collection $\left(b^{S}\right)_{S \subseteq N}$ of elements of $I R_{+}^{m}$ is balanced if and only if there exist veto-controlled simple games $w^{r}, r \in R$, and elements $b^{r} \in I R_{+}^{m}, r \in R$, such that (2.19) holds.

\section{Proof:}

('if'-part) The constraint game corresponding to each constraint $j$ equals $v^{j}=\left(b_{j}^{S}\right)_{S \subseteq N}=\sum_{r \in R} b_{j}^{r} w^{r}$ which is a non-negative weighted sum of vetocontrolled simple games. The latter games are balanced according to the Bondareva-Shapley correspondence, implying the balancedness of $v^{j}, 1 \leq$ $j \leq m$. This proves the balancedness of the collection $\left(b^{S}\right)_{S \subseteq N}$.

('only if'-part) For $1 \leq j \leq m$ let $v^{j}$ denote the constraint game $v^{j}(S)=$ $b_{j}^{S}, S \subseteq N$. The assumption is now that all these games are balanced. Thus, there exist a finite collection $\left(w^{j r}\right)_{r \in R_{j}}$ of simple veto-controlled games and non-negative weights $\alpha_{j r}, r \in R_{j}$, such that $v^{j}$ equals $\sum_{r \in R_{j}} \alpha_{j r} w^{j r}$. Let $b^{j r}$ denote the vector $\alpha_{j r} \chi^{\{j\}} \in I R_{+}^{m}$. We obtain

$$
b^{S}=\left(v^{j}(S)\right)_{1 \leq j \leq m}=\left(\sum_{r \in R_{j}} \alpha_{j r} w^{j r}(S)\right)_{1 \leq j \leq m}=\sum_{1 \leq j \leq m} \sum_{r \in R_{j}} w^{j r}(S) b^{j r}
$$

and we conclude, therefore, that the collection is of the desired form, with index set $R=\cup_{1 \leq j \leq m} R_{j}$.

\section{Potters' approach}

The set of production bundles $\left\{x \in I R_{+}^{k}: A x \leq b^{S}\right\}$ can be considered as the set of actions or alternatives of coalition $S$ in the sense that the players in $S$ are free to choose any of these bundles. There are two approaches based on this observation. One involves the so-called multicommodity game and is treated in section 2.4 .

The other approach is introduced in Potters [1987]. For each coalition $S$ an action set $\Theta(S)$ is considered, with $\Theta(S) \subseteq I R^{k}$, the action space. For a so-called evaluating function $f$, which is a real-valued function on the action space, the following game

$$
v^{f}(S)=\max \{f(x): x \in \Theta(S)\}, \quad \emptyset \neq S \subseteq N
$$


is called an optimization game. Of course, the above optimization model $\langle\theta(S))_{S \subseteq N}, f>$ only gives rise to an optimization game if the optimal values in (2.20) are well defined.

Notice that the linear programming games are optimization games. In fact several other classes of games can be treated in the above way. For further information the reader is referred to Potters [1987].

Let us call the collection of action sets $(\Theta(S))_{S \subseteq N}$ balanced if

$$
\sum_{S \in B} \lambda_{S} \theta(S) \subseteq \operatorname{Conv}(\theta(N))
$$

for each balanced family $B$ with balanced weights $\left(\lambda_{S}\right)_{S \in B}$. An optimization model $\left\langle(\Theta(S))_{S \subseteq N}, f\right\rangle$ is said to be linear if the evaluation function $f$ is linear.

Theorem 2.22 (Potters) An optimization game arising from a linear optimization model with balanced collection of action sets is balanced. Furthermore, if an optimization model gives rise to a balanced optimization game, for arbitrary linear evaluating functions, then the collection of action sets is balanced.

Examples of linear optimization models with balanced collection of action sets are the controlled linear programming models with balanced collection of constraint vectors, supplemented with a price vector as the evaluating function. The first part of theorem 2.22 therefore encloses all foregoing theorems concerning the linear production games.

The second part, however, does not imply the equivalence of the balancedness of a collection $\left(b^{S}\right)_{S \subseteq N}$ and the balancedness of each of the games $v^{c}, c \in I R_{+}^{k}$, as defined in (2.17). The first statement implies the second (corollary 2.24) and for the reverse an extra condition is needed (see theorem 2.26).

\section{Other approaches}

We finally mention two related studies using (not necessarily linear) programming problems in which resource constraints are partly controlled by players and coalitions (Dubey and Shapley [1984]) or in which the parameters are controlled by the players (Kalai and Zemel [1982a]).

\subsubsection{Balanced linear programming games}

In this subsection we will introduce a class of linear programming games with a non-empty core, which contains the veto-controlled linear production 
games and, thus, also the (generalized) linear production games. Controlled linear programming models will be considered where the matrix $A$ only fulfils our assumption on page 56 , and, thus, negative coefficients are allowed. Furthermore, we consider collections of constraint vectors which are not necessarily balanced (and thus theorem 2.22 cannot be applied).

Theorem 2.23 Let $c \in I R_{+}^{k}$ be a price vector for which there is an optimal feasible solution for the minimization problem $\min \left\{y \cdot b^{N}: y \geq 0, y^{t} A \geq c^{t}\right\}$, say $\hat{y} \in I R^{m}$, such that the game $v$ defined by

$$
v(S)=\hat{y} \cdot b^{S}, \quad S \subseteq N
$$

is balanced. Then the corresponding linear programming game $v^{\mathcal{C}}$ is balanced.

Proof: According to the Duality Theorem the optimal value $v^{c}(S)$ equals $\min \left\{y \cdot b^{S}: y \geq 0, y^{t} A \geq c^{t}\right\}$, and since both $\hat{y} \geq 0$ and $\hat{y}^{t} A \geq c^{t}$ hold we must have

$$
v^{c}(S) \leq \hat{y} \cdot b^{S}=v(S), \quad S \subseteq N .
$$

Observe that $v^{c}(N)$ equals $v(N)$. This implies that the core of $v$ is contained in the core of $v^{c}$. Hence, balancedness of $v$ thus implies the balancedness of $v^{e}$.

The game $v$ in theorem 2.23 is a non-negative weighted sum of the $|N|$ constraint games and is therefore (totally) balanced, for arbitrary nonnegative vectors $\hat{y}$, whenever the collection of constraint vectors is (totally) balanced, i.e.,

Corollary 2.24 If the collection of constraint vectors is (totally) balanced then the linear programming game is (totally) balanced for each price vector $c \in I R_{+}^{k}$.

The total balancedness version of corollary 2.24 follows immediately from the observation that each subgame $\left\langle S, v_{\mid S}^{\mathrm{c}}\right\rangle$ of the linear programming game $v^{c}$ is again a linear programming game associated to the same matrix $A$, price vector $c$ and the constraint vector collection $\left(b^{T}\right)_{T \subseteq S}$, with $S \subseteq N$. Now the total balancedness of $\left(b^{T}\right)_{T \subseteq N}$ induces the balancedness of $\left(b^{T}\right)_{T \subseteq S}$, implying the balancedness of $\left\langle S, v_{\mid S}^{c}\right\rangle$.

Notice that whenever the production matrix is chosen to be non-negative the corollary converts to the Owen case if an additive collection of constraint vectors is considered, and the corollary converts to the Granot case if totally 
balanced collections of constraint vectors are considered and, furthermore, it converts to the Curiel, Derks, Tijs case if balanced collections are considered.

\section{An example}

The balancedness of the game $v$ in theorem 2.23 , which is a non-negative weighted sum of the constraint games as we mentioned earlier, can even be accomplished by non-balanced constraint games as the following example shows. Let $N$ be the set $\{1,2,3\}$. Consider the 2-dimensional constraint vectors

\begin{tabular}{c|cccc}
$S$ & $\{1,2\}$ & $\{1,3\}$ & $\{2,3\}$ & $N$ \\
\hline$b^{S}$ & $\left(\begin{array}{l}2 \\
0\end{array}\right)$ & $\left(\begin{array}{l}0 \\
2\end{array}\right)$ & $\left(\begin{array}{l}0 \\
0\end{array}\right)$ & $\left(\begin{array}{l}1 \\
1\end{array}\right)$ \\
$v$ & 2 & 2 & 0 & 2
\end{tabular}

where the non-mentioned constraint vectors and worths are considered to be zero. The two constraint games clearly have an empty core and are therefore not balanced. The game $v$, however, defined by (2.21) with $\hat{y}=\left(\begin{array}{l}1 \\ 1\end{array}\right)$, is balanced. Observe that $\hat{y}$ is the only vector with this property, up to a scalar multiplication. For the production matrix $A=\left(\begin{array}{rr}-1 & 2 \\ 2 & -1\end{array}\right)$ and price vector $c=\left(\begin{array}{l}1 \\ 1\end{array}\right)$ the vector $\hat{y}$ clearly fulfils the conditions mentioned in theorem 2.23 (see figure 2.2 where we sketched the corresponding feasible regions). The corresponding linear programming game $v^{\mathfrak{c}}$ equals the game $v$, and is, therefore, balanced.

\section{Stable price vectors}

With respect to a controlled linear programming model a price vector $c \in I R_{+}^{N}$ is called stable if the corresponding linear programming game $v^{c}$ is balanced. Recapitulating theorem 2.23 we conclude that in a controlled linear programming model a price vector $c$ is stable if there exists an optimal feasible solution $\hat{y} \in I R^{m}$ for $\min \left\{y \cdot b^{N}: y \geq 0, y^{t} A \geq c^{t}\right\}$ such that the game $v=\left(\hat{y} \cdot b^{S}\right)_{S \subseteq N}$ is balanced. Unfortunately, the reverse does not hold, i.e., the reverse of theorem 2.23 is not true. The following example shows this. 


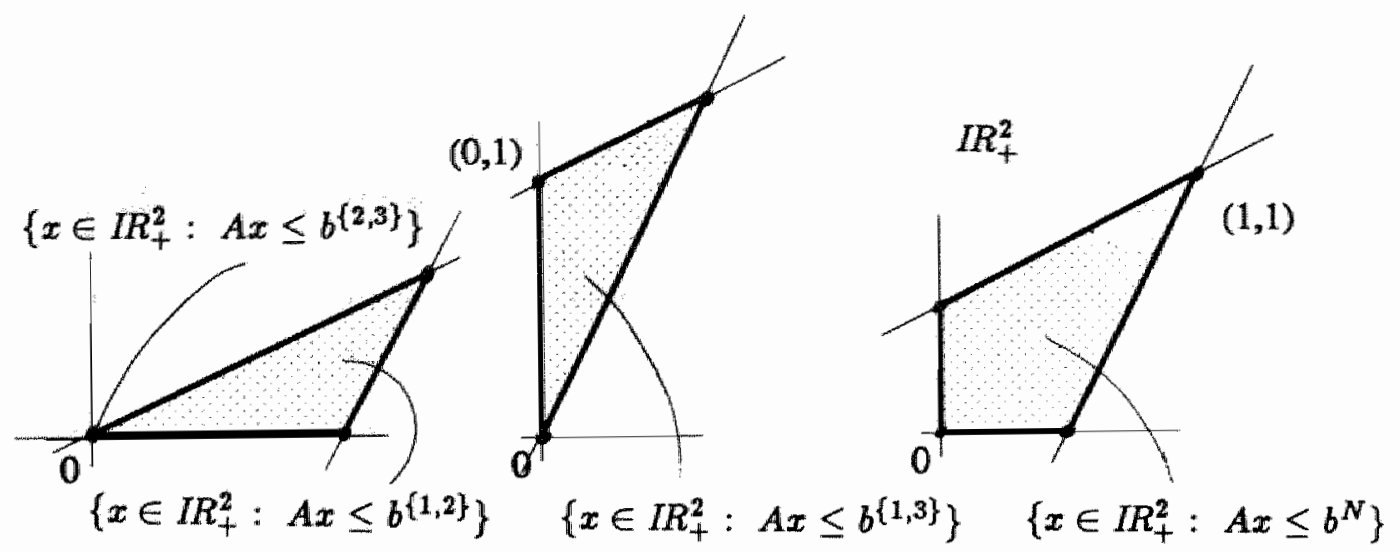

Figure 2.2: The feasible solutions

Consider the controlled linear programming model with player set $N=$ $\{1,2\}$, matrix $A=\left(\begin{array}{lll}1 & 1 & 2 \\ 1 & 2 & 1\end{array}\right)$ and constraint vectors

\begin{tabular}{c|ccc}
$S$ & $\{1\}$ & $\{2\}$ & $N$ \\
\hline$b^{S}$ & $\left(\begin{array}{l}3 \\
5\end{array}\right)$ & $\left(\begin{array}{l}5 \\
3\end{array}\right)$ & $\left(\begin{array}{l}7 \\
7\end{array}\right)$
\end{tabular}

Both constraint games are not balanced and each non-negative weighted sum of these games again is not balanced. In spite of these observations there still exist stable price vectors. For example consider the price vector $c=$ $\left(\begin{array}{lll}1 & 1 & 1\end{array}\right)^{t}$. One easily checks that in the optimization problem $\min \left\{y \cdot b^{N}\right.$ : $\left.y \geq 0, y^{t} A \geq c^{t}\right\}(1,0)^{t}$ and $(0,1)^{t}$ are optimal with optimal value $v^{c}(N)=7$. Furthermore, $v^{c}(\{1\})=\min \left\{y \cdot b^{\{1\}}: y \geq 0, y^{t} A \geq c^{t}\right\}=\left(\begin{array}{l}1 \\ 0\end{array}\right) \cdot\left(\begin{array}{l}3 \\ 5\end{array}\right)=3$, and also $v^{c}(\{2\})=3$, yielding $v^{c}(\{1\})+v^{c}(\{2\})<v^{c}(\{1,2\})$. Therefore, $v^{c}$ has a non-empty vore, implying its balancedness.

Sharp constraint vectors

In the examples above the coefficients of the involved constraint vectors cannot be lowered without changing the corresponding feasible region. A 
constraint vector $b$ with this property is called sharp (with respect to the matrix $A$ ), i.e., $b$ is called sharp if for each constraint $j$ there exists a production bundle $x^{j} \in\left\{x \in I R_{+}^{k}: A x \leq b\right\}$ for which the whole amount of resource $j$ is needed: $\left(A x^{j}\right)_{j}=b_{j}$. A constraint for which this property does not hold is called strongly redundant. Strongly redundant constraints in the linear inequality system $(A x \leq b, x \geq 0)$ can be deleted without affecting the set of feasible solutions.

A sharp constraint vector is, thus, a vector without strongly redundant constraints. In general, for arbitrary matrices $A$ and constraint vectors $b$ with non-empty feasible region $\left\{x \in I R_{+}^{n}: A x \leq b\right\}$ a sharp constraint vector $b^{\prime} \leq b$ exists such that the corresponding feasible region $\left\{x \in I R_{+}^{n}: A x \leq b^{\prime}\right\}$ equals the one corresponding to $b$. It is therefore no restriction if we assume that the constraint vectors in theorem 2.23 are sharp. In the case $b^{N}$ is sharp it is even desirable that the other constraint vectors $b^{S}, S \subset N$, are chosen as small as possible, implying sharpness, since this will enlarge the possibility that the game in (2.21) is balanced. We therefore think that the notion of sharpness will play a significant role in the characterization of the stable price vectors, which is still an open problem.

A comprehensive study dealing with (strong) redundancy and methods for locating (strong) redundant constraints is Karwan, Lofti, Telgen and Zionts [1983]. See also Telgen [1979]. The notion of sharpness is discussed in Derks [1989].

\subsubsection{Core allocations of linear programming games}

In the proof of theorem 2.23 we observed that the core of the game $v$ is contained in the core of $v^{c}$. More precisely, we saw that if $\hat{y} \in I R_{+}^{m}, \hat{y}^{t} A \geq c^{t}$, is such that $v^{c}(N)=\hat{y} \cdot b^{N}$ then the game $v=v^{\hat{v}}=\left(\hat{y} \cdot b^{S}\right)_{S \subseteq N}$ majorizes $v^{c}$, with $v^{\vartheta}(N)=v^{c}(N)$, implying Core $\left(v^{\vartheta}\right) \subseteq \operatorname{Core}\left(v^{c}\right)$. Letting $v^{j}, 1 \leq j \leq m$, be the constraint games with respect to the collection $\left(b^{S}\right) S \subseteq N$ we have $v^{2}=\sum_{j=1}^{m} \hat{y}_{j} v^{j}$, and, thus,

$$
\operatorname{Core}\left(v^{c}\right) \supseteq \operatorname{Core}\left(v^{\vartheta}\right)=\operatorname{Core}\left(\sum_{j=1}^{m} \hat{y}_{j} v^{j}\right) \supseteq \sum_{j=1}^{m} \hat{y}_{j} \operatorname{Core}\left(v^{j}\right)
$$

(with the assumption that if one of the summands with positive weight is the empty set then the algebraic sum is the empty set). Actually, the proofs of the theorems $2.18,2.19$ and 2.20 , which can be found in the literature, essentially employ this result although the relation between the cores of the 
constraint games and the linear programming game as described in (2.22) has not been recognized.

Corollary 2.25 The core of a linear programming game encloses the weighted algebraic sum $\sum_{j=1}^{m} y_{j}$ Core $\left(v^{j}\right)$ of the cores of the constraint games, where the weights $\left(y_{j}\right)_{1 \leq j \leq m}$ form an optimal solution of the minimization problem $\min \left\{y \cdot b^{N}: y \geq 0, y^{t} A \geq c^{t}\right\}$.

It is well-known that in general we do not obtain the whole core of $v^{c}$ in this way. In Owen [1975] an extensive discussion of this phenomenon can be found.

In the second example of the previous subsection each of the games $v^{\theta}$ is not balanced, i.e., Core $\left(v^{\theta}\right)=0$, although the linear programming game $v^{c}$ has a non-empty core. This example in fact shows that we may not even have the equality of the core of $v^{c}$ and the (convex hull of the) union of the cores of $v^{y}$, with $y=\left(y_{j}\right)_{1 \leq j \leq m}$ as in the corollary.

\subsection{Multicommodity games}

A multicommodity game, $\mathrm{MC}$ game for short, is a set-valued function $V$ on the set $2^{N}$ of coalitions of the player set $N$, which assigns to each nonempty coalition $S$ a non-empty, comprehensive and compact subset $V(S)$ of the commodity space $I R_{+}^{k}$, with $V(\theta)=\{0\}$ (a set $C$ of $I R_{+}^{k}$ is called comprehensive if the interval $[0, x]$ is a subset of $C$ for each $x \in C$ ). In the one-commodity case $(k=1)$ we may identify the function $V$ with a single-valued function $v$ for which

$$
V(S)=[0, v(S)] \text { for all } S \subseteq N
$$

Of course, $v$ may be considered to be a (non-negative) game. The MC game model is, therefore, a generalization of the one we considered thus far.

The worth $V(S) \subset I R_{+}^{k}$ of a coalition $S$ in an $\mathrm{MC}$ game $V$ is considered as a set of alternatives among which the members in $S$ are free to choose one element in case the members decide to cooperate. This element $x \in I R_{+}^{k}$ corresponds to a bundle of pay-offs, using $k$ different commodities. Such a multidimensional pay-off arises in cases where the utility comparison of the commodities is difficult or only possible after the involved participants, the players, have agreed upon a kind of cooperation. See also Shapley [1959] and Zeleny [1975], where also multidimensional pay-offs are considered in a (distinct) game theoretic context. 
An application is a generalization of the model in the previous section. In the linear programming context of (2.17) a game is constructed using a price vector $c$. Such a price vector may not be known beforehand, for example in situations in which this vector evolves from the cooperation behavior of the players. Furthermore, it may not be convenient or unrealistic to transform the game theoretic situation into a single game as done in (2.17). In these situations one should consider the set of feasible production bundles $\left\{x \in I R_{+}^{k}: A x \leq b^{S}\right\}$ as the worths $V(S)$ of the coalitions $S \subseteq N$; in the case $A$ is non-negative these sets are comprehensive. Let us call the multicommodity game $V$ arising from a controlled linear programming model $\left\langle A,\left(b^{S}\right)_{S \subseteq N}>\right.$ a linear production $M C$ game, i.e.,

$$
V(S)=\left\{x \in I R_{+}^{k}: A x \leq b^{S}\right\} \text { for each } S \subseteq N .
$$

\subsubsection{Balanced multicommodity games}

In Derks and Tijs [1985] (totally) balanced multicommodity games are considered. A multicommodity game $V$ with player set $N$ is balanced if for each balanced collection $B$ with balanced weights $\left(\lambda_{S}\right)_{S \in B}$ we have

$$
\sum_{S \in B} \lambda_{S} V(S) \subseteq V(N)
$$

One easily proves that this is equivalent to the condition that for each nonnegative (price) vector $c \in I R^{k}$ the game $v^{c}$ defined by

$$
v^{c}(S)=\max \{c \cdot x: x \in V(S)\}, S \subseteq N,
$$

must be balanced. Observe that for a linear production $\mathrm{MC}$ game we have balancedness if and only if for each price vector $c \geq 0$ the corresponding linear programming game is balanced. According to corollary 2.24 we therefore have a balanced linear production $\mathrm{MC}$ game if the corresponding collection $\left(b^{S}\right)_{S \subseteq N}$ is balanced. This result can also be found in Van den Nouweland, Aarts and Borm [1989]. They gave a counterexample of the reverse assertion. However, they used non-sharp constraint vectors, and in fact we have

Theorem 2.26 Let $\left(b^{S}\right)_{S \subseteq N}$ be a collection of sharp constraint vectors with respect to a non-negative matrix $A$. The corresponding linear production $M C$ game is balanced if and only if $\left(b^{S}\right)_{S \subseteq N}$ is balanced.

Proof: The 'if'-part has already been discussed above (sharpness is not needed). Now suppose that $\left(b^{S}\right)_{S \subseteq N}$ is not balanced; then there is a constraint $j$ and a balanced collection $B$ with balanced weights $\left(\lambda_{S}\right)_{S \in B}$ such 
that $\sum_{S \in B} \lambda_{s} b_{j}^{S}>b_{j}^{N}$. For each $S \in B$ there is an element $x^{S}$ of $\left\{x \in I R_{+}^{k}\right.$ : $\left.A x \leq b^{S}\right\}$ with $\left(A x^{S}\right)_{j}=b_{j}^{S}$. Then

$$
\left(A\left(\sum_{S \in B} \lambda_{S} x^{S}\right)\right)_{j}=\sum_{S \in B} \lambda_{S}\left(A x^{S}\right)_{j}=\sum_{S \in B} \lambda_{S} b_{j}^{S}>b_{j}^{N}
$$

i.e., $\sum_{S \in B} \lambda_{S} x^{S}$ is not an element of $V(N)=\left\{x \in I R_{+}^{k}: A x \leq b^{N}\right\}$. This implies that $V$ is not balanced.

We remark that this theorem is actually an instance of theorem 2.26.

Van den Nouweland, Aarts and Borm [1989] considered the total balancedness property. Of course, theorem 2.26 can be naturally extended to this case as well.

\subsubsection{Multicommodity core allocations}

The following notion generalizes the allocation concept to the multicommodity situation. A set-valued function $X$ on $N$ with values subsets of the commodity space $I R_{+}^{m}$ is called a (multicommodity or MC) allocation if $X(i)$ (also denoted by $X_{i}$ ) is comprehensive and compact.

For a multicommodity game $V$ an allocation $X$ is called a $(M C)$ core allocation if the total distribution $\sum_{j \in N} X_{j}$ is a subset of $V(N)$, and the worth $V(S)$ for each coalition $S \subset N$ is contained in $\sum_{j \in S} X_{j}$ as a subset. In general, a comprehensive set cannot be easily decomposed into a sum of non-trivial comprehensive sets unless, of course, only sets of equal shape are used. Furthermore, it might not be possible to extend an allocation $X$ with $\sum_{j \in N} X_{j} \subset V(N)$ to an allocation $X^{\prime}$ with $X_{i} \subseteq X_{i}^{\prime}, i \in N$, and $\sum_{j \in N} X_{j}^{\prime}=V(N)$. Therefore, we allow a core allocation of a $\mathrm{MC}$ game $V$ to distribute only a subset of $V(N)$. See also figure 2.3 where an example is given of a two-person two-commodity game with only one core allocation $X(1)=V(\{1\})$ and $X(2)=V(\{2\})$, which doesnot distribute the whole commodity set of $N$ among the players.

Examples of multicommodity games with core allocations are the balanced games which equal a (finite) sum of games of the form $(w(S) X)_{S \subseteq N}$, with $w$ a veto controlled simple game and $X$ a comprehensive and compact commodity set. Not all $\mathrm{MC}$ games with core allocations, however, can be represented in this way. Figure 2.3 constitutes a counter example. Therefore, theorem 2.8 , stating that a game with non-empty core is a non-negative weighted sum of simple veto-controlled games, cannot be generalized. This is also the case if balanced games are considered in stead of games with 

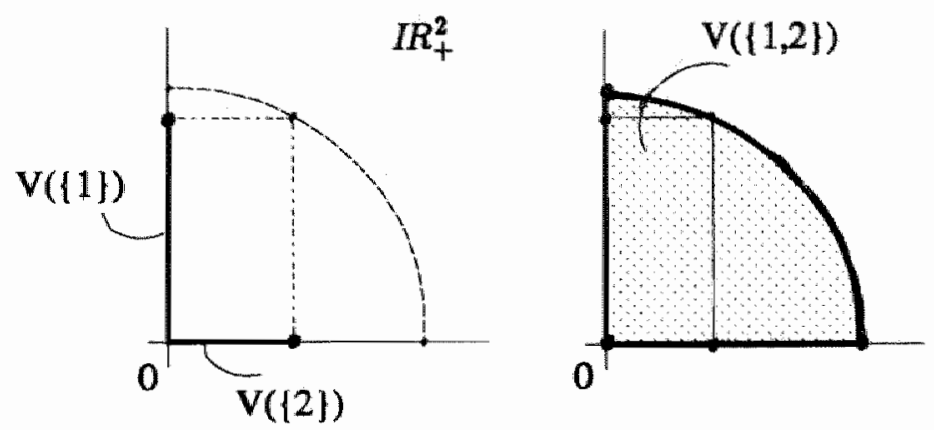

Figure 2.3: A multicommodity game with one core allocation

non-empty core, i.e., there exist balanced multicommodity games having no core allocations. The following example shows this.

A counter example

Let $V$ be the two-commodity game on the player set $N=\{1,2,3\}$ defined by (see also figure 2.4).

$$
\begin{aligned}
V(\{1\}) & =\{(0,0)\}=V(\{2\})=V(\{3\}), \\
V(\{1,2\}) & =\left\{x \in I R_{+}^{2}: x_{1} \leq 1, x_{2}=0\right\} \\
V(\{1,3\}) & =\left\{x \in I R_{+}^{2}: x_{1}^{2}+x_{2}^{2} \leq 1\right\} \\
V(\{2,3\}) & =\left\{x \in I R_{+}^{2}: x_{1}=0, x_{2} \leq 1\right\}, \text { and } \\
V(N) & =\frac{1}{2} V(\{1,2\})+\frac{1}{2} V(\{1,3\})+\frac{1}{2} V(\{2,3\}) .
\end{aligned}
$$

One easily verifies that $V$ is totally balanced. Suppose $X$ is a core allocation of this game. If $X_{2} \neq\{(0,0)\}$, then $X_{2}+V(\{1,3\})$ contains elements with a coordinate greater than 1 , which is impossible since it is contained in $V(N)$ as a subset. Therefore, $X_{2}=\{(0,0)\}$, implying $(1,0) \in X_{1}$ and $(0,1) \in X_{3}$ in order to obey the inclusions $V(S) \subseteq \sum_{j \in S} X_{j}$ for the two-person coalitions $S$. This implies $(1,1) \in X_{1}+X_{2}+X_{3}$ which is not an element of $V(N)$, contradicting the assumption that $X$ is a core allocation. We conclude that no core allocations exist for this game. 



Figure 2.4: A totally balanced two-commodity game

\subsubsection{The quasi-additive multicommodity games}

With respect to a multicommodity allocation $X$ we associate the so-called additive MC game $U^{X}$ in the usual way:

$$
U^{X}(S)=\sum_{i \in S} X_{i} \quad \text { for each coalition } S
$$

Of course, additive MC games are totally balanced. This introduces a new type of totally balanced MC game together with the observation that an intersection of totally balanced MC games is again totally balanced: a multicommodity game is called quasi-additive if it can be represented as a finite intersection of additive MC games.

\section{The one-commodity quasi-additive games}

In the one-commodity case a quasi-additive garne is in fact the minimum of a finite collection of non-negative additive games (a minimum of a finite collection of vectors is to be interpreted coordinate-wise). Kalai and Zemel [1982a] proved that quasi-additive games are exactly the non-negative totally balanced games. Here a similar characterization is given for all totally balanced games.

Theorem 2.27 (Kalai, Zemel) A game is totally balanced if and only if it is the minimum of a finite collection of additive games. 
Proof:

('if'-part) One easily shows that the minimum of a finite collection of (totally) balanced games is again (totally) balanced.

('only if'-part) Consider a totally balanced game $v$, and let $M$ be the the maximum of the differences $v(T)-v(S), S \subseteq T \subseteq N$. The subgame $\left\langle S, v_{\mid S}>\right.$ of $v$ has a non-empty core, say $x^{S} \in \operatorname{Core}\left(v_{\mid S}\right) \subset I R^{S}$. For each non-empty coalition $S \subseteq N$ let $w^{S}$ denote the additive game defined by

$$
w^{S}(T)=\sum_{i \in S \cap T} x_{i}^{S}+|T \backslash S| M, \quad T \subseteq N .
$$

Then $v \leq w^{S}$ since

for each coalition $T \subseteq S$ :

$$
v(T)=v_{\mid S}(T) \leq \sum_{i \in T} x_{i}^{S}=w^{S}(T) ;
$$

and for each coalition $T \nsubseteq S$ :

$$
\begin{aligned}
v(T) & =v(T)-v(T \cap S)+v(T \cap S) \\
& \leq M+\sum_{i \in S \cap T} x_{i}^{S} \\
& \leq|T \backslash S| M+\sum_{i \in S \cap T} x_{i}^{S} \\
& =w^{S}(T) .
\end{aligned}
$$

Let the game $w$ denote the minimum of the collection $\left(w^{s}\right)_{S \subseteq N}$ of additive games. Then $v \leq w$. We obtain $v=w$ since for each coalition $T$ we have $v(T)=x^{T}(T)=w^{T}(T) \geq w(T)$. Thus, $v$ is written as the minimum of a finite collection of additive games.

\section{The quasi-additive MC games}

Unfortunately, the above theorem cannot be extended to the multicommodity case, i.e., not each totally balanced multicommodity game can be expressed as a finite intersection of additive multicommodity games. The example of the two-commodity totally balanced game $V$ as defined on page 67 shows this: suppose, on the contrary, that $\left\{X^{r}: r \in R\right\}$ is a finite collection of two-commodity allocations with

$$
V(S)=\bigcap_{r \in R} U^{X^{r}}(S) \text { for all coalitions } S .
$$


First we note that if for an index $r$ the set $U^{X^{r}}(N)=X_{1}^{r}+X_{2}^{r}+X_{3}^{r}$ contains an element $(1+\delta, 0)$ for a scalar $\delta>0$, then from $(1+\delta, 0) \cup V(N) \subseteq U^{X^{r}}(N)$ and the convexity of $U^{X^{r}}(N)$ it follows that $U^{X^{r}}(N)$ contains an element $\left(1, \frac{1}{2}+\epsilon\right)$, with $\epsilon>0$ suitably small. Because $V(N)=n_{r \in R} U^{X^{r}}(N)$ and $|R|<\infty$ there must be an index $k \in R$ such that

$$
U^{X^{k}}(N) \cap\left\{x \in I R_{+}^{2}: x_{1} \geq 1\right\}=\left\{x \in I R_{+}^{2}: x_{1}=1, x_{2} \leq \frac{1}{2}\right\} .
$$

From $(2.24)$ and $\{(1,0)\}+X_{3}^{k} \subseteq V(\{1,2\})+X_{3}^{k} \subseteq U^{X^{k}}(N)$ it follows that

$$
X_{3}^{k} \subseteq\left\{x \in I R_{+}^{2}: x_{1}=0, x_{2} \leq \frac{1}{2}\right\}
$$

Similarly, from (2.24) and $\{(1,0)\}+X_{2}^{k} \subseteq V(\{1,3\})+X_{2}^{k} \subseteq U^{X^{k}}(N)$ we conclude that

$$
X_{2}^{k} \subseteq\left\{x \in I R_{+}^{2}: x_{1}=0, x_{2} \leq \frac{1}{2}\right\}
$$

From (2.25), (2.26), and

$$
V(\{2,3\}) \subseteq X_{2}^{k}+X_{3}^{k} \subseteq\left\{x \in I R_{+}^{2}: x_{1}=0, x_{2} \leq 1\right\}=V(\{2,3\})
$$

we obtain $X_{2}^{k}=X_{3}^{k}=\left\{x \in I R_{+}^{2}: x_{1}=0, x_{2} \leq \frac{1}{2}\right\}$. Since $(1,0) \in V(N) \subseteq$ $U^{X^{k}}(N)=X_{1}^{k}+\left\{x \in I R_{+}^{2}: x_{1}=0, x_{2} \leq 1\right\}$ the set $X_{1}^{k}$ contains $(1,0)$ and, therefore, $U^{X^{k}}(N)$ contains $(1,0)+(0,1)=(1,1)$, which is in contradiction with (2.24). Thus, (2.23) cannot hold.

Without proof we state the totally balanced MC games $V$ for which the values $V(S), S \subseteq N$, are polyhedral sets in $I R_{+}^{k}$, are examples of quasiadditive MC games.

\subsection{Flow games}

\subsubsection{A multicommodity flow model}

Consider the following capacitated (directed) flow model consisting of a node set $P,|P| \geq 2$, with two distinct nodes $s$ and $t$, the source and the sink, and a set-valued capacity function $C$ on the arc set $P \times P$ with values comprehensive and compact subsets of the commodity space $I R_{+}^{k}$. It is assumed that the transportation of a commodity bundle $x \in I R_{+}^{k}$ from node $p$ to node $q$ along the arc $(p, q)$ is only possible if $x \in C(p, q)$. 
Flows

A function $f: P \times P \longrightarrow I R_{+}^{k}$ is called a flow (from $s$ to $t$ ) if the conservation conditions

$$
\sum_{q \in P} f(p, q)=\sum_{q \in P} f(q, p) \text { for all nodes } p \in P \backslash\{s, t\}
$$

hold, and the net outflow

$$
\nu(f)=\sum_{q \in P} f(s, q)-\sum_{q \in P} f(q, s)
$$

in the source is non-negative. $\nu(f)$ is called the flow value. A flow $f$ is feasible if $f(p, q) \in C(p, q)$ for all $p, q \in P$. A feasible flow $f$ is to be regarded as a schedule to transport the commodity bundle $\nu(f)$ from node $s$ to node $t$ along the arcs, such that all capacity constraints are met. The conservation conditions assure that no flow is lost in the intermediate (transhipment) nodes.

The commodity bundle $\nu(f)$ is not only the net outflow of $s$ but it also equals the net inflow of the sink $t$ as one may have expected. To show this let $f(Q, R)$ denote the sum $\sum_{q \in Q, r \in R} f(q, r)$, with $Q$ and $R$ arbitrary subsets of $P$. We have

$$
f(Q, P)=f(P, Q)+\nu(f)
$$

if $Q$ is a set of nodes containing $s$ and not containing $t$. A set with this property is called a cut. Then, for an arbitrary cut $Q$ we have

$$
\begin{aligned}
f(Q, P \backslash Q) & =f(Q, P)-f(Q, Q) \\
& =f(P, Q)+\nu(f)-f(Q, Q) \\
& =f(P \backslash Q, Q)+\nu(f) .
\end{aligned}
$$

In particular, for the cut $P \backslash\{t\}$ we have $\nu(f)=f(P,\{t\})-f(\{t\}, P)$. Notice that $f(P,\{t\})-f(\{t\}, P)$ is the net inflow of the sink $t$.

\section{The flow capacity}

The flow capacity of the capacitated flow model $\Gamma=\langle P, s, t, C\rangle$ is denoted by $V^{\Gamma}$ and consists of all those commodity bundles $x$ for which there is a feasible flow with flow value $x$. One easily shows that $V^{\Gamma}$ is nonempty $\left(0 \in V^{\mathbb{T}}\right)$, compact and comprehensive.

From (2.27) it follows that for a feasible flow $f$ its flow value is majorized by $f(Q, P \backslash Q)$, with $Q$ an arbitrary cut. The feasibility of $f$ implies $f(Q, P \backslash Q) \in \sum_{q \in Q, p \in P \backslash Q} C(q, p)$; the latter algebraic sum of capacity sets 


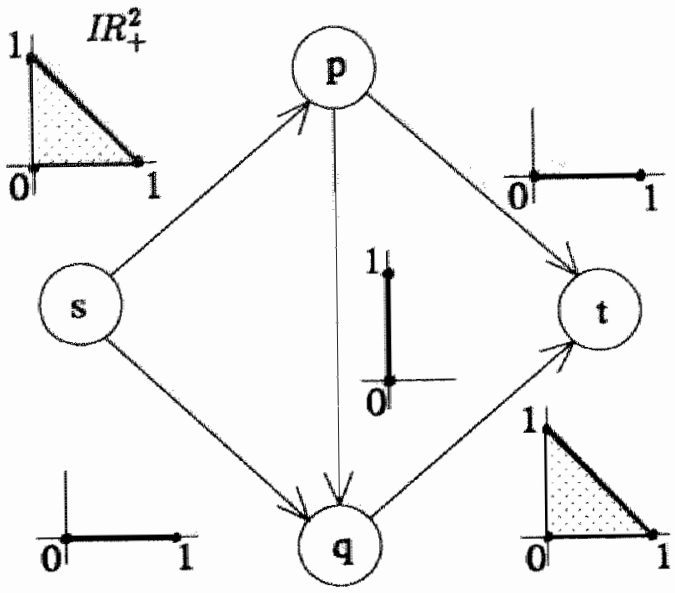

Figure 2.5: A two commodity flow model

is called the capacity of the cut $Q$; it is a comprehensive and compact subset of the commodity space. Therefore, $\nu(f)$ is an element of the capacity of $Q$, i.e.,

Lemma 2.28 The flow capacity is a subset of the capacity of a cut.

\section{An example}

In general the flow capacity unequals the (finite) intersection of the cut capacities. The following example shows this.

Consider the following flow model $\Gamma$ with node set $P=\{s, p, q, t\}$ and capacity function $C$ :

$$
\begin{aligned}
& C(s, p)=\left\{x \in I R_{+}^{2}: x_{1}+x_{2} \leq 1\right\}, \\
& C(s, q)=\left\{x \in I R_{+}^{2}: x_{1} \leq 1, x_{2}=0\right\}=\left[0, x^{\{1\}}\right], \\
& C(p, q)=\left\{x \in I R_{+}^{2}: x_{1}=0, x_{2} \leq 1\right\}=\left[0, x^{\{2\}}\right], \\
& C(p, t)=\left\{x \in I R_{+}^{2}: x_{1} \leq 1, x_{2}=0\right\}=\left[0, \chi^{\{1\}}\right], \text { and } \\
& C(q, t)=\left\{x \in I R_{+}^{2}: x_{1}+x_{2} \leq 1\right\} .
\end{aligned}
$$

The capacities of the other arcs are considered to be $\{0\}$. See also figure 2.5 (arcs with capacity $\{0\}$ are not displayed). There are four cuts, $\{s\},\{s, p\}$, $\{s, q\}$, and $\{s, p, q\}$ with capacities

$$
C(s, p)+C(s, q)=\left\{x \in I R_{+}^{2}: x_{1}+x_{2} \leq 2, x_{2} \leq 1\right\},
$$




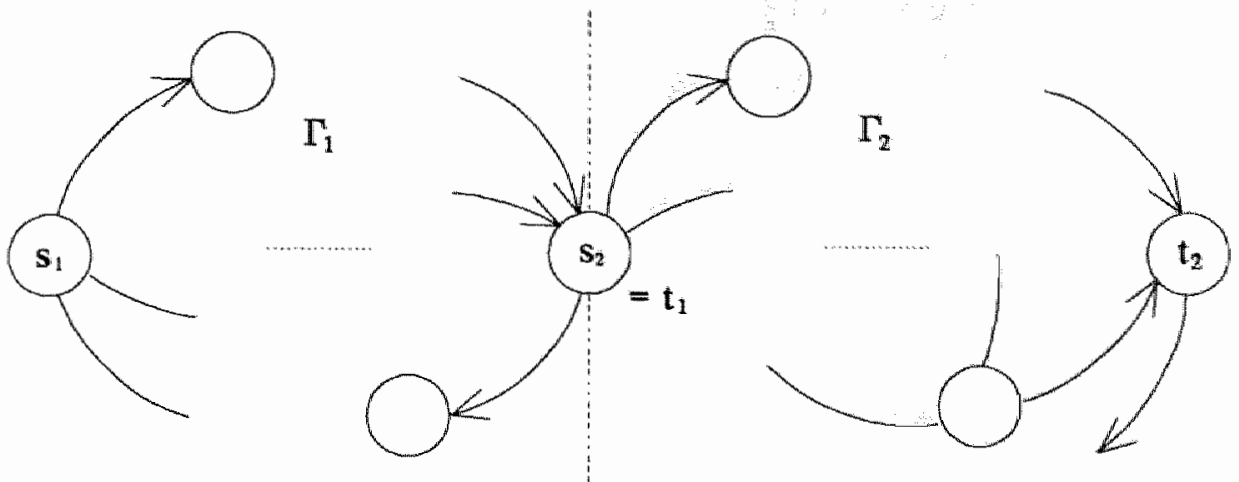

Figure 2.6: Serial composition of flow models

$$
\begin{aligned}
C(s, q)+C(p, t)+C(p, q) & =\left[0,2 \chi^{\{1\}}+\chi^{\{2\}}\right], \\
C(s, p)+C(q, t) & =\left\{x \in I R_{+}^{2}: x_{1}+x_{2} \leq 2\right\}, \text { and } \\
C(p, t)+C(q, t) & =\left\{x \in I R_{+}^{2}: x_{1}+x_{2} \leq 2, x_{2} \leq 1\right\}
\end{aligned}
$$

respectively. The intersection of these sets equals $\left\{x \in I R_{+}^{2}: x_{1}+x_{2} \leq\right.$ $\left.2, x_{2} \leq 1\right\}$. The flow capacity of this flow model can be traced as follows. Let $f$ be a feasible flow with flow value $x$. Notice that we must have $x_{2}=$ $f_{2}(s, p)=f_{2}(p, q)=f_{2}(q, t)$. Therefore, $x_{1}=f_{1}(s, p)+f_{1}(s, q)=f_{1}(s, p)+$ $f_{1}(q, t) \leq 2\left(1-x_{2}\right)$. Thus, $V^{\Gamma} \subseteq\left\{x \in I R_{4}^{2}: x_{1} \leq 2\left(1-x_{2}\right)\right\}$ (in fact equality holds). It follows that the flow capacity $V^{\Gamma}$ unequals the intersection of the cut capacities.

\section{A composition of flow models}

In figure 2.6 we informally describe a possible way to compose one new flow model from two distinct flow models $\Gamma_{1}$ and $\Gamma_{2}$ (with disjoint node sets). In the new flow model $\Gamma$ the sink $t_{1}$ of $\Gamma_{1}$ and the source $s_{2}$ of $\Gamma_{2}$ is contracted into one node. Of course, the arcs between nodes of the two flowmodels $\Gamma_{1}$ and $\Gamma_{2}$ obtain capacity $\{0\}$ in $\Gamma$. It is easily seen that the capacity set $V^{\Gamma}$ of the flow model $\Gamma$ obtained by combining $\Gamma_{1}$ and $\Gamma_{2}$ as in figure 2.6 equals the intersection of $V^{\Gamma_{1}}$ and $V^{\Gamma_{2}}$.

\section{The one-commodity case}

For the one-commodity case each involved capacity is in fact a closed interval in the commodity space $I R_{+}$of the form $[0, c]$ and it may therefore 
be identified with the single vallue $c$. In accordance with this identification we state the following well-known equality.

Theorem 2.29 (Ford, Fulkerson) In the one-commodity case $k=1$ the maximal value of a feasible flow equals the minimal capacity of a cut.

It is also known as the Max-Flow-Min-cut theorem and is introduced in Ford and Fulkerson [1956]; there, it is actually proved by a construction of a feasible flow with flow value equal to the capacity of one of the cuts. In fact, the Max-Flow-Min-cut theorem is an instance of the Duality Theorem (see Dantzig and Fulkerson [1956]). It was followed by many studies on generalizations of this theorem to situations in which simultanuous flows or more commodities are involved. For a comprehensive survey of the obtained results the reader is referred to Assad [1978] and Kennington [1978].

\subsubsection{The flow game}

We extend the flow model with a set $N$ of players and a so-called control function $W$ on the arc set $P \times P$, with values simple games on the player set $N$. It is assumed that the players in $N$ control the arcs in the sense that transportation of a commodity bundle $x \in C(p, q) \backslash\{0\}$ from node $p$ directly to node $q$ is only allowed for coalitions with worth 1 in the simple game $W(p, q)$ (also denoted by $W_{p, q}$ ).

A flow game arising from this controlled flow model is the set-valued function on $2^{N}$ assigning to a coalition $S$ all those flow values of flows $f$ fulfilling the $S$-feasibility conditions

$$
f(p, q) \in W_{p, q}(S) C(p, q)=\left\{\begin{array}{cl}
\{0\} & \text { if } W_{p, q}(S)=0, \\
C(p, q) & \text { if } W_{p, q}(S)=1,
\end{array} \text { for all } p, q \in P\right.
$$

Actually, a flow game is a multicommodity game. This follows from the comprehensiveness and compactness of the flow capacity sets. In the onecommodity case the flow game may be identified with a (single-valued) savings game. Study on the subject of these flow games started in Kalai and Zemel [1982b]. Multicommodity flow games are introduced in Derks and Tijs [1985].

\section{The one-commodity flow game}

In Kalai and Zemel [1982b] the arcs in the one-commodity flow model are controlled by a single player. We will refer to this kind of control as the dictatorial control. As we saw earlier it can be modelled by a control function $W$ with values only among the dictator games $u^{\{i\}}, i \in N$. 
Theorem 2.30 (Kalai, Zemel) A flow game arising from a dictatorial controlled one-commodity flow model is totally balanced. Furthermore, for each non-negative totally balanced game $v$ there exists a dictatorial controlled onecommodity flow model for which $v$ is the flow game.

In view of theorem 2.27 the following theorem describes a generalization to the multicommodity case. Its proof uses similar arguments as the one for theorem 2.30 in Kalai and Zemel [1982b].

Theorem 2.31 A flow game arising from a dictatorial controlled flow model is totally balanced. Furthermore, for each quasi-additive multicommodity game $V$ there exists a dictatorial controlled flow model for which $V$ is the flow game.

Proof: Let $V$ be a flow game corresponding to a flow model with node set $P$ and control function $W$. Let $i_{p, q}$ denote the player $i$ for which $W(p, q)=u_{\{i\}}$. Let $\lambda$ be a balanced vector. We, thus, have $\sum_{S: i_{p, q} \in S \subseteq N} \lambda_{S}=1$ for all $p, q \in P$. In order to prove $\sum_{\emptyset \neq S \subseteq N} \lambda_{S} V(S) \subseteq V(N)$ let $f^{S}$ be an $S$-feasible flow for each non-empty coalition $S$. Obviously, $f=\sum_{\emptyset \neq S \subseteq N} \lambda_{S} f^{S}$ is a flow. The $N$-feasibility of $f$ follows from the observation that $f^{S}(p, q)=0$ for each coalition $S$ not containing the player $i_{p, q}$, which fully controls the arc $(p, q)$; therefore,

$$
f(p, q)=\sum_{\emptyset \neq S \subseteq N} \lambda_{S} f^{S}(p, q)=\sum_{i p, q \in S \subseteq N} \lambda_{S} f^{S}(p, q),
$$

which is a convex combination of elements of the capacity set $C(p, q)$. Since the capacity sets are convex we, thus, have $f(p, q) \in C(p, q)$ for all $p, q \in P$, implying the feasibility of $f$. Feasibility and $N$-feasibility are equivalent since in the simple games $W(p, q), p, q \in P$, the grand coalition is always winning: $W_{p, q}(N)=1$. We conclude that $\nu(f) \in V(N)$. Now $\nu(f)=\nu\left(\sum_{\emptyset \neq S \subseteq N} \lambda_{S} f^{S}\right)=\sum_{\emptyset \neq S \subseteq N} \lambda_{S} \nu\left(f^{S}\right)$, and, therefore, each element of $\sum_{\emptyset \neq S \subseteq N} \lambda_{S} V(S)$ is contained in $V(N)$. This proves the balancedness of $V$. The total balancedness follows from the observation that each subgame of $V$ is again a flow game from a dictatorial controlled flow model.

The second assertion in the theorem is shown by construction. Let $V$ be a multicommodity game which equals the intersection of the additive $\mathrm{MC} \mathrm{ga-}$ mes corresponding to a finite number of $\mathrm{MC}$ allocations $X^{1}, X^{2}, \ldots, X^{R}$. Consider the flow model $\Gamma_{r}$ consisting of the node set $P^{r}=\left\{s_{r}, t_{r}\right\} \cup$ $N=\left\{s_{r}, t_{r}, 1,2, \ldots, n\right\}$, and capacity function $C^{r}$ defined by $C^{r}\left(s_{r}, i\right)=$ 
control: $u^{\{1\}}$
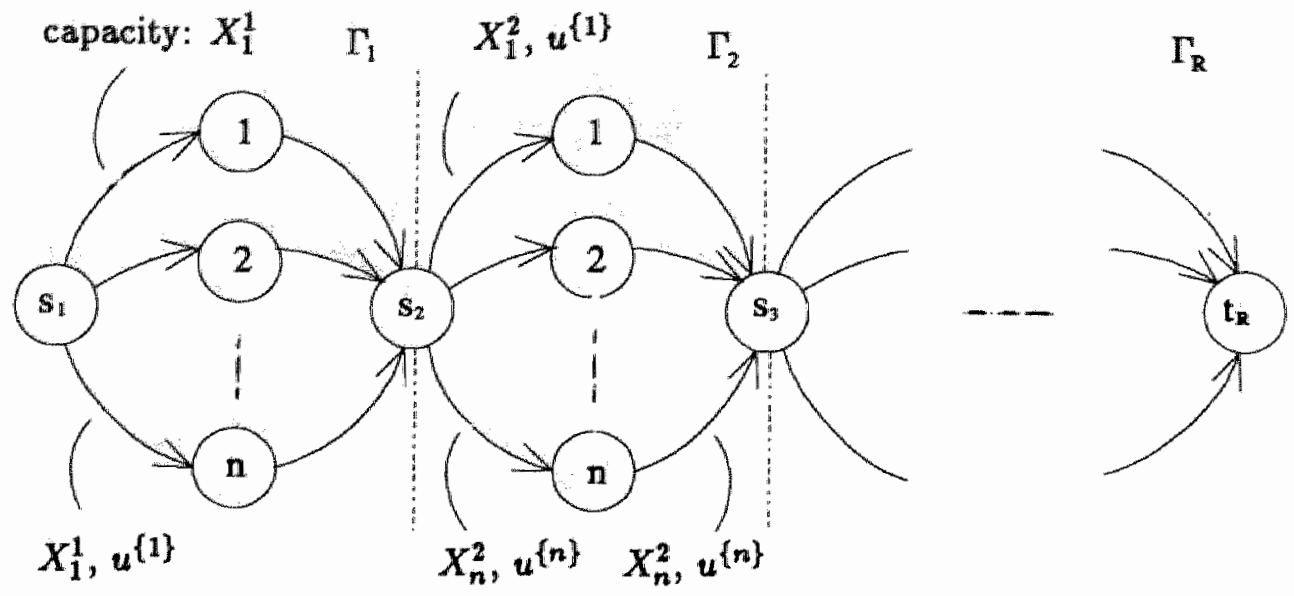

Figure 2.7: A controlled flow model corresponding to an intersection of $R$ additive multicommodity games

$C^{r}\left(i, t_{r}\right)=X^{r}(i)$ for each $i \in N$, and $C^{r}(p, q)=\{0\}$ otherwise, and a dictorial control function $W^{r}$, with $W^{r}(s, i)=W^{r}(i, t)=u^{\{i\}}$ for each $i \in N$. Of course, the flow game arising from $\Gamma_{r}$ is the additive MC game corresponding to the allocation $X^{r}$. Now construct the dictatorial controlled flow model $\Gamma$ by combining the $|R|$ flow models $\Gamma_{r}, r \in R$, in the same way as in figure 2.7. The flow game $V^{\Gamma}$ equals the intersection of the flowgames $V^{\Gamma}$, $r \in R$, which equal the additive MC games corresponding to $X^{r}, r \in R$. We conclude that $V^{\Gamma}=V$.

\subsubsection{Multicommodity games arising from veto-controlled flow models}

Let us call a controlled capacitated flow model veto-controlled if the values of the control function consist of veto-controlled simple games. In Curiel, Derks, Tijs [1989] the following generalization of theorem 2.30 is given.

Theorem 2.32 (Curiel, Derks, Tijs) A flow game arising from a vetocontrolled one-commodity flow model is balanced. Furthermore, for each balanced game $v \in \mathbf{G}_{+}^{N}$ there exists a veto-controlled one-commodity flow model for which $v$ is the flow game. 
The proof follows a similar reasoning as the one in the proof of theorem 2.31 , combined with the characterization of a non-negative balanced game as a non-negative weighted sum of veto-controlled simple games (theorem 2.8).

In the remainder of this section we will discuss which multicommodity games actually arise from veto-controlled capacitated flow models. Our main result is that there exist balanced two-commodity games which cannot be represented as arising from a veto controlled capacitated flow model. This is done by the introduction of the so-called strict balancedness property for MC games and by showing that this property holds for all two-commodity flow games. These notion and result are also discussed in Derks and Tijs [1986].

We will, thus, show that the second part of theorem 2.32 cannot be generalized. The generalization of the first part, however, is possible and is described in the following theorem. A proof follows a similar reasoning as the first part of the proof of theorem 2.31, and is, therefore, omitted.

Theorem 2.33 A multicommodity flow game arising from a veto-controlled multicommodity flow model is balanced.

\section{strict balancedness}

A multicommodity game $V$ with player set $N$ is strictly balanced in an element $y$ of the commodity space $I R_{+}^{k}$ if for each balanced family $B \subseteq$ $2^{N} \backslash\{\emptyset\}$ with corresponding balanced weights $\left(\lambda_{S}\right)_{s \in B}$ and elements $y^{S} \in$ $V(S), S \in B$, with $y=\sum_{S \subseteq N} \lambda_{S} y^{S}$, the following holds: there exists an $\epsilon>0$ such that

$$
y+x-y^{S} \in V(N) \text { for all } S \in B \text { and } x \in V(S) \text { with }\left\|x-y^{S}\right\|<\epsilon .
$$

\section{Strictly balanced multicommodity games}

$V$ is called strictly balanced if it is strictly balanced in each element of $V(N)$. Notice that strict balancedness implies balancedness. Furthermore, a balanced MC game $V$ is strictly balanced in each element of $V(N)$ which is not Pareto-optimal(for a comprehensive and closed subset $C$ of $I R_{+}^{k}$ an element $y \in C$ is Pareto-optimal if $\{y\}=C \cap\left(\{y\}+I R_{+}^{k}\right)$ ). For an interpretation of the notion of strict balancedness the following example will be illustrative.

example

Consider the two-commodity game which is defined on page 67 . The Pareto optimal element $y=\left(1, \frac{1}{2}\right)$ of $V(\{1,2,3\})$ equals the combination 
$\frac{1}{2} y^{\{1,2\}}+\frac{1}{2} y^{\{1,3\}}+\frac{1}{2} y^{\{2,3\}}$, with $y^{\{1,2\}}=y^{\{1,3\}}=(1,0)$ and $y^{\{2,3\}}=(0,1)$. In order to have strict balancedness in $y$ there must be an $\epsilon>0$ such that $y+x-y^{\{1,3\}} \in V(\{1,2,3\})$ for each $x \in V(\{1,3\})$ within distance $\epsilon$ from $y^{(1,3)}$. Let $x^{e}$ denote the element $\left(\sqrt{1-\frac{1}{4} \epsilon^{2}}, \frac{1}{2} \epsilon\right) \in V(\{1,3\})$. We obviously have $\left\|x-y^{\{1,3\}}\right\|<\epsilon$ and $y+x^{\epsilon}-y^{\{1,3\}}=\left(\sqrt{1-\frac{1}{4} \epsilon^{2}}, \frac{1}{2}+\frac{1}{2} \epsilon\right)$, which strictly majorizes the Pareto-optimal element $\left(\frac{1}{2}+\frac{1}{2} \sqrt{1-\epsilon^{2}}, \frac{1}{2}+\frac{1}{2} \epsilon\right)$ of $V(\{1,2,3\})$, i.e, $y+x^{\varepsilon}-y^{\{1,3\}} \notin V(\{1,2,3\})$. This proves that $V$ is not strictly balanced in $y=\left(1, \frac{1}{2}\right)$.

One easily shows that the additive $\mathrm{MC}$ games are strictly balanced. Also the polyhedral multicommodity games $V$ arising from a balanced game $v_{\text {, }}$ i.e., there is a positive normal vector $\mathrm{p} \in I R_{+}^{k}$ such that $V(S)=\left\{x \in I R_{+}^{k}\right.$ : $p \cdot x \leq v(S)\}$ for each non-empty coalition $S$, are strictly balanced. Furthermore, the class of strictly balanced $\mathrm{MC}$ games is closed under addition and intersection. We conclude that each quasi-additive or balanced polyhedral MC game is strictly balanced. It also follows that a balanced multicommodity game $V$ is strictly balanced for which $V(N)$ is polyhedral.

\section{Strictly convex sets}

A convex set $C$ is called strictly convex if for each convex combination $x=\lambda y+(1-\lambda) z$, with $y, z \in C \backslash\{x\}$, there is a scalar $\delta>0$ such that for each $y^{\prime} \in C$, with $\left\|y^{\prime}-y\right\|<\delta$ we have $x+y^{\prime}-y \in C$.

Examples of strictly convex sets are the open convex sets, the convex sets of dimension at most 2 , and the polyhedral sets. In figure 2.8 a 3dimensional convex set is displayed which is not strictly convex. We will only prove that

\section{Lemma 2.34 $A$ convex set $C \subseteq I R^{2}$ is strictly convex.}

Proof: Consider the convex combination $z=\lambda y+(1-\lambda) z$, with $y, z \in$ $C \backslash\{x\}$. If $x$ is an interior point of $C$ then a scalar $\delta>0$ obviously exists such that for each $y^{\prime} \in C$, with $\left\|y^{\prime}-y\right\|<\delta$, we have $x+y^{\prime}-y \in C$. Thus suppose $x$ is a border element of $C$. Let $p \in I R^{2}$ be such that $p \cdot c \leq p \cdot x$ for all $c \in C$. The convexity of $C$ implies that $p \cdot y$ and $p \cdot z$ equals $p \cdot x$. Let $\delta \geq 0$ be such that $c \in C$ whenever $p \cdot c \leq p \cdot x$ and $\|c-x\| \leq \delta$. If $\delta$ cannot be chosen to be positive then $C$ is a subset of the line through $x, y$ and $z$; in this case strict convexity is easily shown. Thus, let $\delta>0$. Then for each $y^{\prime} \in C$, with $\left\|y^{\prime}-y\right\|<\delta$, we have $p \cdot\left(x+y^{\prime}-y\right)=p \cdot y^{\prime} \leq p \cdot x$ and $\left\|\left(x+y^{\prime}-y\right)-x\right\|=\left\|y^{\prime}-y\right\| \leq \delta$. Thus, $x+y^{\prime}-y \in C$. We conclude that $C$ is strictly convex. 




Figure 2.8: a convex set which is not strictly convex

Let us now describe our main result.

Theorem 2.35 A flow game arising from a veto-controlled flow model with strictly convex capacity sets is strictly balanced.

Proof: Let $V$ be a flow game arising from the flow model with node set $P$, control function $W$, and capacity function $C$ such that $C(p, q)$ is a strictly convex subset of $I R_{+}^{k}$ for each $p, q \in P$.

Suppose $V$ is not strictly balanced in an element $y \in I R_{+}^{k}$. Thus, there exist a balanced family $B$ of coalitions, with balanced weights $\left(\lambda_{S}\right)_{S \in B}$, elements $y^{S} \in V(S), S \in B$, with $y=\sum_{S \in B} \lambda_{S} y^{S}$, and there is a coalition $T \in B$, and a sequence $x^{1}, x^{2}, \ldots$ of elements of $V(T)$, with $\lim _{r \rightarrow \infty} x^{r}=y^{T}$ and

$$
y+x^{r}-y^{T} \notin V(N) \text { for all } r=1,2, \ldots \text {. }
$$

Let $f^{1}, f^{2}, \ldots$ be a sequence of $T$-feasible flows such that $\nu\left(f^{r}\right)=x^{r}, r=$ $1,2, \ldots$. The sequence $f^{1}(p, q), f^{2}(p, q), \ldots$ must have an accumulation point in $C(p, q)$ since its elements are contained in the compact set $C(p, q)$, with $p, q \in P$ arbitrary. Since $P$ is finite there is a subsequence $f^{r_{1}}, f^{r_{2}}, \ldots$ such that $\lim _{k \rightarrow \infty} f^{r_{k}}(p, q)$ exists for each arc $(p, q)$. We may assume that this subsequence equals the original sequence. Let $f^{T}$ be the function on the set $P \times P$ of arcs such that

$$
f^{T}(p, q)=\lim _{r \rightarrow \infty} f^{r}(p, q) \quad \text { for all } p, q \in P .
$$


Then $f^{T}$ is a feasible flow. In fact, $f^{T}$ is $T$-feasible since $f^{T}(p, q)=0$ for all $p, q \in P$ with $W_{p, q}(T)=0$. The flow value of $f^{T}$ obviously equals $\lim _{T \rightarrow \infty} \nu\left(f^{r}\right)$, i.e., $\nu\left(f^{T}\right)=y^{T}$. For the other coalitions $S$ in $B$ let $f^{S}$ be an $S$-feasible flow with value $y^{S}$. Then $f=\sum_{S \in B} \lambda_{S} f^{S}$ is a feasible flow with value $y$, which is therefore an element of $V(N)$.

For an arbitrary arc $(p, q)$, which is controlled by coalition $T$, i.e., the worth of $T$ in the simple game $W(p, q)$ equals 1 , consider the commodity bundle

$$
z=\frac{1}{\left(1-\lambda_{T}\right)} \sum_{s \in B \backslash\{T\}} \lambda_{S} f^{s}(p, q) .
$$

Then $z$ is a non-negative combination of elements of $C(p, q)$. Now $f^{S}(p, q) \neq$ 0 only for coalitions $S \in B$ with $W_{p, q}(S)=1$, yielding

$$
z=\frac{1}{\left(1-\lambda_{T}\right)} \sum_{S \in B \backslash\{T\}} \lambda_{S} W_{p, q}(S) f^{S}(p, q) .
$$

Using that the simple games $W(p, q), p, q \in P$, are veto-controlled, and, therefore, balanced, we obtain that the sum of the weights in the decomposition of $z$ is majorized by 1 :

$$
\begin{aligned}
\frac{1}{\left(1-\lambda_{T}\right)} \sum_{S \in B \backslash\{T\}} \lambda_{S} W_{p, q}(S) & =\frac{1}{\left(1-\lambda_{T}\right)}\left(\sum_{S \in B} \lambda_{S} W_{p, q}(S)-\lambda_{T}\right) \\
& \leq \frac{1}{\left(1-\lambda_{T}\right)}\left(W_{p, q}(N)-\lambda_{T}\right) \\
& =1,
\end{aligned}
$$

i.e., $z$ is majorized by a convex combination of elements of $C(p, q)$. Since the capacity sets are comprehensive we conclude that $z$ is an element of $C(p, q)$. Of course, $f(p, q)=\sum_{S \in B} \lambda_{S} f^{S}(p, q)$ equals the convex combination (1$\left.\lambda_{T}\right) z+\lambda_{T} f^{T}$ of elements of $C(p, q)$, which is strictly convex. This implies that there is a $\delta_{p, q}>0$ such that

$$
f(p, q)+x-f^{T}(p, q) \in C(p, q) \text { for } x \in C(p, q) \text { with }\left\|x-f^{T}(p, q)\right\|<\delta_{p, q} \text {. }
$$

Let $\delta>0$ denote the minimum of the scalars $\delta_{p_{0} q}$, with $p, q \in P$ such that $W_{p, q}(T)=1$. Furthermore, let $M$ be such that $\left\|f^{r}(p, q)-f^{T}(p, q)\right\|<\delta$ for each $r>M$ and $p, q \in P$. Using (2.29) we conclude that the function $g^{r}=f+f^{r}-f^{T}$ must be a feasible flow whenever $r>M$. Thus, $\nu\left(g^{r}\right)=$ $y+x^{r}-y^{T} \in V(N)$ if $r>M$; a contradiction. Our assumption that $V$ is 
not strictly balanced in $y$ must therefore be false.

According to lemma 2.34 all capacity sets of a two-commodity flow model are strictly convex. Therefore,

Corollary 2.36 A two-commodity flow game is strictly balanced.

\subsubsection{Concluding remarks}

Combining corollary 2.36 and the example of a non-strictly balanced twocommodity game on page 67 we conclude that the class of balanced multicommodity games properly contains the flow games. This shows that a full generalization of the one-commodity case in theorem 2.32 is not possible.

Now let TBMC denote the family of totally balanced multicommodity games, FMC the family of flow games, QAMC the family of quasi-additive MC games and PMC the family of totally balanced polyhedral MC games. We have

$$
\text { PMC } \subset \text { QAMC } \subseteq \text { FMC } \subset \text { TBMC. }
$$

Open is: coincides QAMC with FMC?

Or: is it possible to express a flow game as a finite intersection of additive multicommodity games?

If the answer is affirmative then this will imply that the strict balancedness is not a sufficient condition for a multicommodity game to assure that it is a flow game. To see this, consider the two-commodity game $V^{\prime}$ with $V^{\prime}(S)=V(S)$ for each coalition $S \neq\{1,3\}$, where $V$ is defined on page 67 , and $V^{\prime}(\{1,3\})=\left\{x \in I R_{+}^{2}: x_{1} \leq 1, x_{2}=0\right\}$. $V^{\prime}$ is strictly balanced but in a similar way as on page 69 one shows that $V^{\prime} \notin$ QAMC. 


\section{Chapter 3}

\section{Cones of semibalanced games and related classes}

\subsection{Introduction}

Chapter 3 focusses on the characterization of several classes of semibalanced games and related classes, by presenting generating sets which are representative if possible. Besides the direct methods, we will use the method as presented in the theorems 1.17 and 1.18, where a linear mapping, with the game space as the image, gives rise to classes of games, for which generating sets are described. We will show here that the semibalanced games and the maximally blocked games can be treated in this way. Also results are obtained for the 1-convex games and the clan games. Other methods, constructive in nature, will be used in order to obtain generating sets for the class of semiconvex games and the class of quasi-balanced games.

contents

In section 3.2 we will study the cones $\mathbf{S}^{N}, \mathbf{S}_{0}^{N}$ and $\mathbf{S}_{+}^{N}$ of semibalanced games, followed by a discussion of cones of games for which (some of) the maximal blockedness conditions hold. These cones are the class of maximally blocked games and the class of 1-convex games, their restrictions to the 0 -normalized games and the non-negative games, and the class of clan games. Section 3.4 is devoted to the characterization of the cones $\mathrm{SC}^{N}$ and $\mathrm{SC}_{0}^{N}$ of semiconvex games, and section 3.5 to the cones $\mathrm{Q}^{N}$ and $\mathrm{Q}_{0}^{N}$ of quasi-balanced games. As we have mentioned the proofs of our results are constructive in nature. However, for the quasi-balanced games we will explicitly present a procedure representing a quasi-balanced game as a non- 
negative weighted sum of the games in a finite generating set of $Q^{N}$. For the other types of games similar procedures follow immediately from the proofs.

The concluding section 3.6 starts with a summary of our results in this chapter. We will show in subsection 3.6.2 that several of the constructed generating sets in fact span the whole game space, implying that the corresponding classes are fully dimensional.

The gap function defines a 1-1 mapping between cones of 0-normalized games and cones of cost games with zero marginal contribution allocation. The cones of cost games corresponding to the considered cones of 0-normalized games will be discussed in subsection 3.6.3.

\subsection{Cones of semibalanced games}

Recall that a game $v$ is semibalanced if the additive game corresponding to its marginal contribution allocation $b^{v}=(v(N)-v(N \backslash\{j\}))_{j \in N}$ majorizes $v$, i.e., $v(S) \leq \sum_{j \in S} b_{j}^{v}$ for $\emptyset \neq S \subseteq N$.

\subsubsection{The cone of semibalanced games}

The cone $\mathrm{S}^{N}=\left\{v \in \mathrm{G}^{N}: v(S) \leq \sum_{j \in S} b_{j}^{v}\right.$ for each $\left.\emptyset \neq S \subseteq N\right\}$ obviously encloses the subset $\mathbf{D}^{N}$ of games for which the marginal contribution allocation is a core allocation. The negative of the simple game $\hat{w}$, defined by

$$
\hat{w}=\mathbf{1}^{N}+\sum_{j \in N} \mathbf{1}^{N \backslash\{j\}}
$$

is also semibalanced since $-\hat{w}$ is non-positive with marginal contribution allocation $b^{-\hat{w}}=-b^{\hat{w}}=0$. Clearly, $-\hat{w}$ is not an element of $\mathrm{D}^{N}$.

According to the following theorem $\mathbf{S}^{N}$ can be decomposed into $\mathbf{D}^{N}$ and the cone generated by $-\hat{w}$.

Theorem 3.1 The class $\mathbf{S}^{N}$ of semibalanced games equals the algebraic sum of the cone $\mathbf{D}^{N}$ and Cone $(\{-\hat{w}\})$.

Proof: First notice that $\mathbf{D}^{N}+\operatorname{Cone}(\{-\hat{w}\}) \subseteq \mathbf{S}^{N}$ holds since $\mathbf{S}^{N}$ is a cone. Now let $v \in \mathbf{S}^{N}$ and $\gamma^{v}$ be the marginal gap $\sum_{j \in N} b_{j}^{v}-v(N) \geq 0$. We will demonstrate that the game $w=v+\gamma^{v} \hat{w}$ is semibalanced. First, note that $b^{w}=b^{v}+\gamma^{v} b^{w}=b^{v}$. Furthermore,

$$
\sum_{j \in N} b_{j}^{w}=\sum_{j \in N} b_{j}^{v}=v(N)+\gamma^{v}=w(N) .
$$


Therefore, using (2.2) concerning the equality of the marginal gap and $\sum_{j \in N \backslash\{i\}} b_{j}^{u}-v(N \backslash\{i\})$ for arbitrary $v \in \mathbf{G}^{N}$ and player $i$, we have also

$$
\sum_{j \in N \backslash\{i\}} b_{j}^{w}=w(N \backslash\{i\}) \quad \text { for each player } i \in N .
$$

Moreover,

$$
\sum_{j \in S} b_{j}^{w}=\sum_{j \in S} b_{j}^{v}=\sum_{j \in S} b_{j}^{v}+\gamma^{v} \hat{w}(S) \geq v(S)+\gamma^{v} \hat{w}(S)=w(S)
$$

for all coalitions $S$ with less than $|N|-1$ players. We conclude that the additive game associated to $b^{w}$ majorizes $w$. This implies the semibalancedness of $w$.

Together with $\sum_{j \in N} b_{j}^{w}=w(N)$ we, therefore, obtain that $w$ is an element of $\mathbf{D}^{N}$. Now the game $v$ equals $w-\gamma^{v} \hat{w}$ which is an element of $\mathbf{D}^{N}+\operatorname{Cone}(\{-\hat{w}\})$, and we conclude that $\mathbf{S}^{N} \subseteq \mathbf{D}^{N}+\operatorname{Cone}(\{-\hat{w}\})$. The theorem now follows.

\section{A generating set}

Note that the game $-\hat{w}$ is not contained in $\mathbf{D}^{N}$ since $-\hat{w}$ does not possess a core allocation. Adding $-\hat{w}$ to the generating set of $\mathbf{D}^{N}$, mentioned in theorem 2.2, provides us with a generating set of $\mathbf{S}^{N}$.

Corollary 3.2 The cone of semibalanced games is generated by the $2^{|N|}+$ $|N|-1$ games $u^{\{i\}}$ and $-u^{\{i\}}, i \in N,-1 s, S \subset N$, with $|S| \leq|N|-2$, and $-\hat{w}$.

The decomposition of a semibalanced game into a game in $\mathbf{D}^{N}$ and a non-negative multiple of $-\hat{w}$ in the proof of theorem 3.1 is unique. To see this let $u^{0}-\alpha^{0} \hat{w}$ and $u^{1}-\alpha^{1} \hat{w}$ be two different decompositions of the same semibalanced game, with $u^{0}, u^{1} \in \mathbf{D}^{N}$. We obtain that $\hat{w}$ is a linear combination of the games that generate $\mathbf{D}^{N}$, say

$$
\hat{w}=\sum_{j \in N} \alpha^{\{j\}} u^{\{j\}}+\sum_{S \subset N,|S| \leq|N|-2} \alpha^{S}\left(-\mathbf{1}^{S}\right) .
$$

Using the linearity of the marginal contribution allocation we must have $0=$ $b^{凶}=\sum_{j \in N} \alpha^{\{j\}} b^{u^{(j)}}+\sum_{S \subset N,|S| \leq|N|-2} \alpha^{s^{s}} b^{-1^{s}}=\sum_{j \in N} \alpha^{\{j\}} \chi^{\{j\}}$, implying that $\alpha^{\{j\}}=0, j \in N$. Therefore, $\hat{w}$ is a linear combination of the games $-\mathbb{1}^{S}, S \subset N,|S| \leq|N|-2$, a contradiction. 
As we shall see in subsection 3.6 .2 , the games in theorem 3.1 except the additive games $-u^{\{i\}}, i \in N$, form a base of the game space.

The decomposition $v=\left(v+\gamma^{v} \hat{w}\right)-\gamma^{v} \hat{w}$ uniquely splits the game $v$ in a part which has a (unique) satisfactory core allocation and in a part corresponding to the only game without core allocations in the generating set of $\mathbf{S}^{N}$, given in corollary 3.2. In this context the marginal gap $\gamma^{v}$ may be seen as a sort of dissatisfaction factor for a semibalanced game and in Driessen [1988] it is proposed to divide this factor equally among the players. One, thus, obtains the following solution scheme on the semibalanced games:

$$
s(v)=b^{v}-\frac{1}{|N|}\left(\sum_{j \in N} b_{j}^{v}-v(N)\right) \chi^{N} \quad \text { with } v \in \mathbf{S}^{N} .
$$

This scheme has the property that it is majorized by the marginal contribution allocation, a property that also holds for core allocations. It is a natural property in the context of our reasoning on page 11. Notice that the allocation $s(v)$ equals the average of the extreme elements of the marginal core MCore $(v)$.

\section{The 0-normalized semibalanced games}

Of course, $\mathbf{S}^{N}$ cannot have extreme directions since the subspace of additive games $\mathbf{A}^{N}=\operatorname{Cone}\left(\left\{u^{\{i\}}: i \in N\right\} \cup\left\{-u^{\{i\}}: i \in N\right\}\right)$ is included in $\mathbf{S}^{N}$. The cone of 0 -normalized semibalanced games, however, is pointed as follows from the fact that a semibalanced game $v$, for which also $-v$ is semibalanced, is necessarily an additive game. Thus, $\mathbf{S}_{0}^{N}$ is generated by a representative set of its extreme directions (theorem 1.14). Consider the decomposition $v=v+\gamma^{v} \hat{w}-\gamma^{v} \hat{w}$ again, with $\gamma^{v}=\sum_{j \in N} b_{j}^{v}-v(N)$. Observe that $-\hat{w}$ is 0-normalized; thus, whenever $v$ is a 0 -normalized semibalanced game then $v+\gamma^{v} \hat{w}$ must be contained in $\mathbf{D}_{0}^{N}$. A representative set of extreme directions of the cone $D_{0}^{N}$ is presented in theorem 2.2. The games in this set and $-\hat{w}$ therefore generate $\mathbf{S}_{0}^{N}$. In fact these games are also extreme.

Theorem 3.3 The cone $\mathrm{S}_{0}^{N}$ is generated by its extreme directions $u^{\{i\}}-\mathbb{1}^{\{i\}}$, $i \in N,-\mathbb{1}^{S}, 2 \leq|S| \leq|N|-2$, and $-\hat{w}$.

Proof: The only fact to be proved is the extremality of the mentioned games. The extremality of $-\mathbb{1}^{S}$, with $S \subset N, 2 \leq|S| \leq|N|-2$, follows from the same observation as is used for these games in the proof of theorem 2.5. A similar reasoning holds also for $-\hat{w}$.

Now let $W$ denote the set consisting of the mentioned games in theorem 3.3. Then $\mathbf{S}_{0}^{N}=$ Cone(W). Suppose that for a player $i$ the game $u^{\{i\}}-$ 
$1^{\{i\}}$ is a non-negative weighted sum of the games in $W$, say $w^{\{i\}}-\mathbb{1}^{(i\}}=$ $\sum_{w \in W} \alpha^{w} w$. Then

$$
\chi^{\{i\}}=b^{u^{(i)}-\mathbf{1}^{(i)}}=\sum_{w \in W} \alpha^{w} b^{w}=\sum_{j \in N} \alpha^{w^{(j)}-\mathbf{1}^{(j)}} \chi^{\{j\}}
$$

and this only holds in case $\alpha^{u^{(i)}-\mathbf{1}^{(i)}}=1$ and $\alpha^{\mathrm{u}^{(i)}-\mathbf{1}^{(j)}}=0$ for the other players $j \in N \backslash\{i\}$. Thus, the non-negative weighted sum

$$
\sum_{S \subset N, 2 \leq|S| \leq|N|-2} \alpha^{-\mathbf{1}^{s}}\left(-\mathbb{1}^{s}\right)+\alpha^{-\hat{w}}(-\hat{w})
$$

of the non-positive games in $W$ equals the all-losing game, yielding that the involved weights must be zero. We conclude that $u^{\{i\}}-\mathbb{1}^{\{i\}}$ cannot be represented as a non-negative weighted sum of the other games in $W$. This implies the extremality of $u^{\{i\}}-\mathbf{1}^{\{i\}}$.

The results in this subsection can also be derived with the help of theorem 1.17. This will be shown in the next subsection, where the theorems 1.17 and 1.18 are used in order to construct a generating set of the class $\mathbf{S}_{+}^{N}$ of non-negative semibalanced games.

\subsubsection{The cone of non-negative semibalanced games}

Consider the following mapping on the $(|N|+1)$-dimensional vector space $I R^{N} \times I R$ :

$$
(x, \gamma) \mapsto u^{x}-\gamma \hat{w}
$$

This mapping is clearly linear. The game $u^{x}-\gamma \hat{w}$ has a marginal contribution allocation equal to $x$ and its marginal gap equals $\sum_{j \in N} x_{j}-u^{z}(N)+\gamma \hat{w}(N)=$ $\gamma$. Observe that a garne $v$ equals $u^{b^{*}}-\gamma^{v} v_{\text {one }}$ on the coalitions in $\Omega=$ $\{N\} \cup\{N \backslash\{i\}, i \in N\}$ since for a player $i$ we have

$$
\begin{aligned}
v(N \backslash\{i\}) & =\sum_{j \in N \backslash\{i\}} b_{j}^{u}-\left(\sum_{j \in N} b_{j}^{v}-v(N)\right)=\left(u^{b^{v}}-\gamma^{v} \hat{w}\right)(N \backslash\{i\}), \text { and } \\
v(N) & =\sum_{j \in N} b_{j}^{v}-\left(\sum_{j \in N} b_{j}^{\nu}-v(N)\right)=\left(u^{b^{v}}-\gamma^{\nu} \hat{w}\right)(N) .
\end{aligned}
$$

From $\sum_{j \in S} b_{j}^{v}=u^{b^{v}}(S)=\left(u^{b^{v}}-\gamma^{v} \hat{w}\right)(S)$ we conclude that $v$ is semibalanced if and only if

$$
\gamma^{v} \geq 0, \text { and } v \leq u^{b^{v}}-\gamma^{v} v \text { one. }
$$


Therefore, we can apply theorem 1.17 with $f$ the mapping as defined in (3.1) with domain $D=I R^{N} \times I R_{+}$, and the set $\Omega$ containing the grand coalition $N$ and the $(|N|-1)$-person coalition. From the above observations we derive that the cone $W^{N}$ as defined in theorem 1.17 equals $\mathrm{S}^{N}$.

Consider also the cones $D_{x}$, and $D_{p}$, with $D_{z}=\left\{(x, \gamma): f^{(x, \gamma)}(\{i\}) \geq 0\right.$ for all $i \in N\}$, and $D_{p}=\left\{(x, \gamma): f(x, \gamma) \in \mathbf{G}_{+}^{N}\right\}$ (see theorem 1.17.B and C respectively). The equalities $D_{z}=I R_{+}^{N} \times I R_{+}$and

$$
D_{p}=\left\{(x, \gamma) \in I R_{+}^{N} \times I R_{+}: \sum_{j \in N \backslash\{i\}} x_{j} \geq \gamma \text { for each } i \in N\right\}
$$

follow immediately. Generating sets for $D, D_{z}$, and $X_{p}$ are

$$
\begin{aligned}
X & =\left\{\left(\chi^{\{i\}}, 0\right): i \in N\right\} \cup\left\{\left(-\chi^{\{i\}}, 0\right): i \in N\right\} \cup\{(0,1)\}, \\
X_{z} & =\left\{\left(\chi^{(i\}}, 0\right): i \in N\right\} \cup\{(0,1)\}, \text { and } \\
X_{p} & =\left\{\left(\chi^{s},|S|-1\right): \emptyset \neq S \subseteq N\right\}
\end{aligned}
$$

respectively. We will show that $X_{p}$ is a generating set of $D_{p}$. Obviously, $X_{p}$ is a subset of the cone $D_{p}$. Now let $(x, \gamma)$ be an element of $D_{p}$. If $\gamma=0$ then $(x, \gamma)$ equals the non-negative combination $\sum_{j \in N} x_{j}\left(\chi^{\{j\}}, 0\right)$ of elements of $X_{p}$. Thus suppose $\gamma>0$. Consider the non-empty set $S=\left\{i \in N: x_{i}>0\right\}$ of players with positive entries in $x$. Choose $\epsilon>0$ such that

- $\epsilon(|S|-1) \leq \gamma$, and

- $\epsilon \chi^{s} \leq x$.

Then $\left(x-\epsilon \chi^{S}, \gamma-\epsilon(|S|-1)\right)=(x, \gamma)-\epsilon\left(\chi^{S},|S|-1\right)$ is a non-negative vector. Furthermore,

$$
\begin{aligned}
\sum_{j \in N \backslash\{i\}}\left(x-\epsilon \chi^{S}\right)_{j} & =\sum_{j \in N \backslash\{i\}} x_{j}-\epsilon|S|+\epsilon \chi_{j}^{S} \\
& \geq \gamma-\epsilon|S|+\epsilon=\gamma-\epsilon(|S|-1) \text { if } i \epsilon S,
\end{aligned}
$$

and

$$
\begin{aligned}
\sum_{j \in N \backslash\{i\}}\left(x-\epsilon \chi^{S}\right)_{j} & =\sum_{j \in N} x_{j}-\epsilon|S| \geq \sum_{j \in N \backslash\{k\}} x_{j}+\epsilon-\epsilon|S| \\
& \geq \gamma-\epsilon(|S|-1) \text { if } i \notin S,
\end{aligned}
$$

where player $k$ is chosen arbitrarily from the coalition $S$. We conclude that $(x, \gamma)-\epsilon\left(\chi^{S},|S|-1\right)$ is contained in the set $D_{p}$. Applying theorem 1.15 we obtain that $X_{p}$ is a generating set of $D_{p}$. 
Observe that if the scalar $\epsilon$ above is chosen as large as possible then one can construct only finitely many slices $\epsilon\left(\chi^{S},|S|-1\right)$ before eventually the zero element turns up. Thus, the derivations above in fact yield a constructive method to represent an element of $D_{p}$ as a non-negative weighted sum of elements of $X_{p}$.

$X_{z}$ and $X_{p}$ are in fact representative sets of extreme directions of $D_{z}$ and $D_{p}$ respectively. Let us show this for $D_{p}$. For an arbitrary non-empty coalition $S$ we consider $\left(\chi^{S},|S|-1\right)$ as a non-negative combination of the elements in $X_{p}$, say $\left(\chi^{S},|S|-1\right)=\sum_{\emptyset \neq T \subseteq N} \alpha^{T}\left(\chi^{T},|T|-1\right)$. Using $0=\chi_{i}^{S}$ for $i \notin S$ we obtain $\alpha^{T}=0$ for coalitions $T$ containing player $i$, and, therefore,

$$
|S|=\sum_{j \in S} \chi_{j}^{S}=\sum_{\emptyset \neq T \subseteq S} \alpha^{T}|T|
$$

and also

$$
|S|-1=\sum_{\emptyset \neq T \subseteq S} \alpha^{T}(|T|-1)=\sum_{\emptyset \neq T \subseteq S} \alpha^{T}|T|-\sum_{\emptyset \neq T \subseteq S} \alpha^{T},
$$

yielding $\sum_{\emptyset \neq T \subseteq S} \alpha^{T}=1$. Combining this result with the fact that for each player $i$ in $S$ we have $1=\chi_{i}^{S}=\sum_{\emptyset \neq T \subseteq S} \alpha^{T} \chi_{i}^{T}=\sum_{i \in T \subseteq S} \alpha^{T}$, we conclude that $\alpha^{S}=1$ and $\alpha^{T}=0$ otherwise. This proves that $\left(\chi^{S},|S|-1\right)$ is extreme. Therefore,

Lemma 3.4 A generating set of $D_{p}=\left\{(x, \gamma) \in I R_{+}^{N} \times I R_{+}: u^{x}-\gamma \hat{w} \in \mathbf{G}_{+}^{N}\right\}$ is formed by the extreme directions $\left(\chi^{S},|S|-1\right), \emptyset \neq S \subseteq N$.

Observe that the mapping in (3.1) is $\Omega$-injective (with $\Omega=\left\{S \in 2^{N}\right.$ : $|S| \geq|N|-2\}$ ); therefore, the conditions, mentioned in theorem 1.18 are satisfied. In order to apply theorem 1.18 we first list the games which correspond to the elements in the generating sets $X, X_{z}$ and $X_{p}$. In the table $i$ is a player, and $S$ denotes a non-empty coalition.

\begin{tabular}{|c|c|c|c|c|}
\hline$(x, \gamma)$ & $u^{x}-\gamma \hat{w}$ & $X$ & $X_{z}$ & $X_{p}$ \\
\hline$\left(\chi^{\{i\}}, 0\right)$ & $u^{\{i\}}$ & & & \\
$\left(-\chi^{\{i\}}, 0\right)$ & $-u^{\{i\}}$ & $\mathrm{x}$ & $\mathrm{x}$ & \\
$(0,1)$ & $-\hat{w}$ & $\mathrm{x}$ & $\mathrm{x}$ & \\
$\left(\chi^{S},|S|-1\right)$ & $\mathrm{car}^{S}-(|S|-1) \hat{w}$ & & & $\mathrm{x}$ \\
\hline
\end{tabular}


It follows that theorem 3.1 concerning a generating set of $\mathbf{S}^{N}$ is an instance of part $A$ of theorem 1.17, with the above definitions of the notions involved. In the same way the representative set of $\mathrm{S}_{0}^{N}$, as displayed in theorem 3.3 , can be obtained by applying theorem 1.17.B and theorem 1.18.A. Applying theorem 1.17.C and theorem 1.18.B we finally obtain (with $\Omega=\{N\} \cup$ $\{N \backslash\{i\}: i \in N\})$.

Theorem 3.5 The class $\mathbf{S}_{+}^{N}$ is generated by the extreme directions

$$
\operatorname{mix}_{\Omega^{\prime}}\left(\operatorname{car}^{S}-(|S|-1) \hat{w}\right), \quad \text { with } \emptyset \neq S \subseteq N \text { and } \Omega^{\prime} \subseteq 2^{N}, \Omega \subseteq \Omega^{\prime}
$$

These games have the following expression:

$\operatorname{mix}_{\Omega^{\prime}}\left(\operatorname{car}^{S}-(|S|-1) \hat{w}\right)(T)=\left\{\begin{array}{cl}1 & \text { if } T=N \text { or } T=N \backslash\{i\}, i \notin S, \\ |S \cap T| & \text { if } T \subset N,|T| \leq|N|-2, T \notin \Omega, \\ 0 & \text { otherwise. }\end{array}\right.$

Observe that there are extreme non-simple semibalanced games.

\subsection{Cones of maximally blocked games}

The marginal core $\operatorname{MCore}(v)=\left\{x \in I R^{N}: \sum_{j \in N} x_{j}=v(N), x \leq b^{v}\right\}$ has been introduced in chapter 1 as a relaxation of the core. It turned out that the subset of 1-convex games $v$ consists precisely of those games for which the core is non-empty and equals MCore( $v$ ) (cf. theorem 1.7). The 1-convex games have been defined as the games $v$ for which $v(N) \leq \sum_{j \in N} b_{j}^{\psi}$ and

$$
v(S) \leq v(N)-\sum_{j \notin S} b_{j}^{\psi} \quad \text { for each coalition } S \neq \emptyset
$$

hold. The games with property (3.2) were called the maximally blocked games.

\subsubsection{The cones of maximally blocked games and 1-convex games}

Similar decompositions of classes of maximally blocked games will be given as is done in the previous section. In the decomposition the airport cost savings game $w^{N}$ plays a significant role, where $w^{N}$ is the game assigning $|S|-1$ to a non-empty coalition $S$. 
Theorem 3.6 The class $\mathrm{MB}^{N}$ of the maximally blocked games equals $\mathbf{D}^{N}+$ $\operatorname{Span}\left(\left\{w^{N}\right\}\right)$, and the class $\mathbf{1 C}^{N}$ of 1 -convex games equals $\mathbf{D}^{N}+\operatorname{Cone}\left(\left\{w^{N}\right\}\right)$.

Proof: Both classes of games are cones containing $\mathbf{D}^{N}$. Furthermore, $w^{N}$ is a 1-convex game and both $w^{N}$ and $-w^{N}$ are maximally blocked. Thus, $\mathrm{D}^{N}+\operatorname{Cone}\left(\left\{w^{N}\right\}\right) \subseteq \mathbf{I C}^{N}$ and $\mathbf{D}^{N}+\operatorname{Span}\left(\left\{w^{N}\right\}\right) \subseteq \mathbf{M B}^{N}$.

Now let $v$ be a maximally blocked game. Consider the game $w=v-$ $\gamma^{v} w^{N}$. Its marginal contribution allocation $b^{w}$ equals $b^{v}-\gamma^{v} b^{w^{N}}=b^{v}-\gamma^{v} \chi^{N}$. The semibalancedness of $w$ now follows from

$$
\begin{aligned}
\sum_{j \in S} b_{j}^{w} & =\sum_{j \in S} b_{j}^{v}-\gamma^{v}|S| \\
& =\sum_{j \in S} b_{j}^{v}-v(S)+v(S)-\gamma^{v} w^{N}(S)-\gamma^{v} \\
& =\sum_{j \in S} b_{j}^{v}-v(S)+w(S)-\left(\sum_{j \in N} b_{j}^{v}-v(N)\right) \\
& =w(S)-\sum_{j \in N \backslash S} b_{j}^{v}-v(S)+v(N) \\
& =w(N) \text { if } S=N, \\
& \geq w(S) \text { if } S \subset N .
\end{aligned}
$$

The latter inequality is a consequence of the maximal blockedness of the game $v$. We conclude that $w$ is an element of $\mathbf{D}^{N}$. Of course, $v$ equals $w+\gamma^{v} w^{N}$ which is contained in $\mathbf{D}^{N}+\operatorname{Cone}\left(\left\{w^{N}\right\}\right)$ whenever $\gamma^{v} \geq 0$. Hence, $\mathbf{1 C}^{N} \subseteq \mathbf{D}^{N}+\operatorname{Cone}\left(\left\{w^{N}\right\}\right)$ and also $\mathrm{MB}^{N} \subseteq \mathbf{D}^{N}+\operatorname{Span}\left(\left\{w^{N}\right\}\right)$.

In theorem 2.2 a generating set for $\mathrm{D}^{N}$ is given. Using the abowe decompositions it follows immediately that

Corollary 3.7 The $2^{|N|}+|N|-1$ games $u^{\{i\}},-u^{\{i\}}, i \in N$, and $-\mathbb{1}^{s}$, $\emptyset \neq S \subset N,|S| \leq|N|-2$, and $w^{N}$ form a generating set for the class of 1 -convex games. The same set, supplemented with $-w^{N}$, generates $\mathrm{MB}^{N}$.

As was also the case in the decomposition of the class $\mathbf{S}^{N}$, the decomposition of a maximally blocked (or 1-convex) game $v$ into the sum of a game of $\mathbf{D}^{N}$ and a multiple of $w^{N}$ is unique. Suppose this was not the case, i.e., there exist $u^{0}, u^{1} \in \mathbf{D}^{N}$ and $\alpha^{0}, \alpha^{1} \in I R_{+}$with $u^{0}+\alpha^{0} w^{N}=u^{1}+\alpha^{1} w^{N}$, then it would imply that $w^{N}$ is a linear combination of elements of $\mathbf{D}^{N}$, say

$$
w^{N}=\sum_{j \in N} \alpha^{j} u^{\{j\}}+\sum_{s: 1 \leq|s| \leq|N|-2} \alpha^{s} 1^{S}
$$


where we used the generating set of $\mathbf{D}^{N}$ in theorem 2.2. Then

$$
b^{w^{N}}=\chi^{N}=\sum_{j \in N} \alpha^{j} b^{u^{(j)}}+\sum_{S: 1 \leq|S| \leq|N|-2} \alpha^{S} b^{\mathbf{1}^{s}}=\sum_{j \in N} \alpha^{j} \chi^{\{j\}},
$$

implying $\alpha^{i}=1$ for each player $i$. Therefore, $w^{N}(N)=\sum_{j \in N} u^{\{j\}}(N)=$ $\operatorname{car}^{N}(N)$, which is clearly a contradiction.

It will be shown in subsection 3.6.2 that the generating set of $1 C^{N}$ in theorem 3.7, without the games $-u^{\{i\}}, i \in N$, forms a base of $\mathrm{G}^{N}$. Therefore, the class of 1-convex games is a full dimensional class of games in the game space (see also Driessen and Tijs [1983]).

\section{The 0-normalized games}

Observe that the 0 -normalizedness of $w^{N}$ implies that for a 0 -normalized game $v$ the game $v-\gamma^{v} w^{N}$ must be contained in $\mathbf{D}_{0}^{N}$. Again using theorem 2.5, where a generating set of extreme directions of $\mathbf{D}_{0}^{N}$ is presented, we obtain generating sets for $\mathbf{1 C}_{0}^{N}$ and $\mathbf{M B}_{0}^{N}$. However, for the latter cone there are no extreme games since it contains the 1-dimensional subspace $\operatorname{Span}\left(\left\{w^{N}\right\}\right)$ as a subset.

Corollary 3.8 The $2^{|N|}-|N|-1$ games $u^{\{i\}}-1^{\{i\}}, i \in N$, and $-\mathbb{1}^{S}$, $\emptyset \neq S \subset N,|S| \leq|N|-2$, and $w^{N}$ are extreme directions and form a generating set for the class of 0-normalized 1-convex games. Furthermore, the same set, supplemented with $-w^{N}$, generates $\mathrm{MB}_{0}^{N}$.

Proof: The only statement left to prove is the extremality of the mentioned games in $\mathbf{1 C}_{0}^{N}$. Except for $w^{N}$ the extremality of these games in the larger class $\mathbf{S}_{0}^{N}$ implies their extremality in $\mathbf{1 C}_{0}^{N}$. In the paragraph following corollary 3.7 we proved that $w^{N} \notin \operatorname{Span}(W)$, with $W$ a generating set of $\mathbf{D}^{N}$. This implies that in particular $w^{N} \notin \mathbf{D}_{0}^{N}$ which proves that this game cannot be written as a non-negative weighted sum of the other games mentioned in the corollary. It must, therefore, be extreme in the cone generated by all these games, i.e., in $\mathbf{1 C}_{0}^{N}$.

We will briefly show in the following subsection that our results here could have been obtained by applying theorem 1.17. The necessary preparations are carried out in order to construct generating sets of the classes $\mathbf{M B}_{+}^{N}$ and $\mathbf{I C}_{+}^{N}$. 


\subsubsection{Cones of non-negative maximally blocked games}

Consider the following linear mapping on the $(|N|+1)$-dimensional vector space $I R^{N} \times I R$ :

$$
(x, \gamma) \mapsto u^{x}-\gamma v_{\text {one }},
$$

where $v_{o n e}$ is the all-winning game assigning 1 to each non-empty coalition. The marginal contribution allocation of the game $u^{x}-\gamma v_{\text {one }}$ clearly equals $x$, and its marginal gap equals $\sum_{j \in N} x_{j}-u^{x}(N)+\gamma v_{\text {one }}(N)=\gamma$.

The cone $\mathrm{MB}_{+}^{N}$ of non-negative maximally blocked games

The maximal blockedness property of $u^{x}-\gamma v_{\text {one }}$ follows from

$$
\begin{aligned}
\left(u^{x}-\gamma v_{\text {one }}\right)(S) & =u^{x}(N)-\sum_{j \notin S} x_{j}-\gamma \\
& =\left(u^{x}-\gamma v_{\text {one }}\right)(N)-\sum_{j \notin S} b_{j}^{u^{x}-\gamma y^{y} \text { one }}
\end{aligned}
$$

with $S \subseteq N$ non-empty.

For an arbitrary game $v$ we have $v \leq u^{b^{*}}-\gamma^{v} v_{\text {one }}$ if and only if $v \in \mathrm{MB}^{N}$; equality holds for the coalitions with at least $|N|-1$ coalitions.

In order to apply theorem 1.17 with $f$ as defined in (3.3), with domain $D=I R^{N} \times I R, \Omega=\left\{S \in 2^{N}:|S| \geq|N|-1 \mid\right\}$, notice that the sets $D_{z}=$ $\left\{(x, \gamma): f^{(x, \gamma)}(\{i\}) \geq 0, i \in N\right\}$, and $D_{p}=\left\{(x, \gamma): f(x, \gamma) \in \mathbf{G}^{N}\right\}$ are equal to $\left\{(x, \gamma): x_{i} \geq \gamma, i \in N\right\}$, and $\left\{(x, \gamma): \sum_{j \in S} x_{j} \geq \gamma, \emptyset \neq S \subseteq N\right\}$ respectively. Generating sets of $D, D_{z}$, and $D_{p}$ are

$$
\begin{aligned}
X & =\left\{\left(\chi^{\{i\}}, 0\right): i \in N\right\} \cup\left\{\left(-\chi^{\{i\}}, 0\right): i \in N\right\} \cup\{(0,1),(0,-1)\}, \\
X_{z} & =\left\{\left(\chi^{\{i\}}, 0\right): i \in N\right\} \cup\left\{\left(\chi^{N}, 1\right),-\left(\chi^{N}, 1\right)\right\}, \quad \text { and } \\
X_{p} & =\left\{\left(\chi^{\{i\}}, 0\right): i \in N\right\} \cup\left\{-\left(\chi^{\{i\}}, 1\right): i \in N\right\} \cup\left\{\left(\chi^{N}, 1\right),-(0,1)\right\}
\end{aligned}
$$

respectively. Let us show that $X_{p}$ is a generating set of the cone $D_{p} . X_{p}$ is a subset of $D_{p}$; furthermore,

if $\gamma \geq 0$ (thus $x \geq 0$ ):

$$
(x, \gamma)=\sum_{j \in N}\left(x_{j}-\gamma\right)\left(\chi^{\{i\}}, 1\right)+\gamma\left(\chi^{N}, 1\right)
$$

if $\gamma<0$ and $x \geq 0$ :

$$
(x, \gamma)=\sum_{j \in N} x_{j}\left(x^{\{i\}}, 0\right)+(-\gamma)(0,-1)
$$


and, finally, if $x \geq 0$ (thus $\gamma<0$ ):

$$
(x, \gamma)=\sum_{j: x_{j} \geq 0} x_{j}\left(\chi^{\{j\}}, 1\right)+\sum_{j: x_{j}<0}\left(-x_{j}\right)\left(-\chi^{\{j\}},-1\right)+\left(\sum_{i: x_{i}<0} x_{i}-\gamma\right)(0,-1)
$$

In the above decompositions the involved weights are non-negative, which implies that $X_{p}$ is a generating set of $D_{p}$.

Before we apply theorem 1.17 consider the following table in which we displayed the games corresponding to the elements of the sets $X, X_{z}$ and $X_{p}$. In the table $i$ denotes a player.

\begin{tabular}{|c|c|c|c|c|}
\hline$(x, \gamma)$ & $u^{x}-\gamma v_{\text {one }}$ & $X$ & $X_{z}$ & $X_{p}$ \\
\hline$\left(\chi^{\{i\}}, 0\right)$ & $u^{\{i\}}$ & $\mathrm{x}$ & $\mathrm{x}$ & $\mathrm{x}$ \\
$\left(-\chi^{\{i\}}, 0\right)$ & $-u^{\{i\}}$ & $\mathrm{x}$ & & \\
$(0,1)$ & $-v_{\text {one }}$ & $\mathrm{x}$ & & \\
$-(0,1)$ & $v_{\text {one }}$ & $\mathrm{x}$ & & $\mathrm{x}$ \\
$\left(\chi^{N}, 1\right)$ & $w^{N}$ & & $\mathrm{x}$ & $\mathrm{x}$ \\
$-\left(\chi^{N}, 1\right)$ & $-w^{N}$ & & $\mathrm{x}$ & \\
$-\left(\chi^{\{i\}}, 1\right)$ & $v_{\text {one }}-u^{\{i\}}$ & & & $\mathrm{x}$ \\
\hline
\end{tabular}

The results in the previous subsection concerning the maximally blocked games now easily follow from theorem 1.17 part $\mathbf{A}$ and part $\mathbf{B}$. Applying part $\mathbf{C}$ we obtain

Theorem 3.9 The cone $\mathrm{MB}_{+}^{N}$ is generated by the extreme directions $\operatorname{mix}_{\Omega^{\prime}}\left(u^{\{i\}}\right), \operatorname{mix}_{\Omega^{\prime}}\left(\right.$ vone $\left.-u^{\{i\}}\right), i \in N$, and $\operatorname{mix}_{\Omega^{\prime}}\left(w^{N}\right), \Omega^{\prime} \subseteq 2^{N}, \Omega \subseteq \Omega^{\prime}$.

Proof: It follows from theorem 1.17.C that the mentioned games form a generating set for $\mathbf{M B}_{+}^{N}$. The extremality of these games follows from theorem 1.18.B, the observation that the mapping in (3.3) is $\Omega$-injective, and the fact that $X_{p}$ consists of extreme directions. The latter assertion can be proven easily.

The cone $1 C_{+}^{N}$ of non-negative 1-convex games

Let us restrict the sets $D, D_{z}$ and $D_{p}$ such that the mapping in (3.3) yields 1 -convex games. The only extra condition for a maximally blocked garne $v$ to be 1-convex is the non-negativity of the marginal gap $\gamma^{v}=$ 
$\sum_{j \in N} b_{j}^{t \prime}-v(N)$ (see page 12) since MCore(v) has to be non-empty. Therefore, a game $u^{x}-\gamma v_{\text {one }}$ is 1 -convex if and only if its marginal gap $\gamma$ is non-negative. Furthermore, a game $v$ is 1 -convex if and only if

$$
\gamma^{v} \geq 0, \text { and } v \leq u^{b^{*}}-\gamma^{v} v_{\text {one }} \text {. }
$$

Let us therefore restrict the sets $D, D_{z}$, and $D_{p}$ in the above derivations to the elements of $I R^{N} \times I R_{+}$. Then $D=I R^{N} \times I R_{+}, D_{z}=\left\{(x, \gamma): x_{i} \geq \gamma \geq\right.$ $0, i \in N\}$, and $D_{p}=\left\{(x, \gamma): \sum_{j \in S} x_{j} \geq \gamma \geq 0, \emptyset \neq S \subseteq N\right\}$. Notice that $D_{p}$ and $D_{z}$ actually coincide. It is clear that the set $X_{p}=\left\{\left(\chi^{\{i\}}, 0\right): i \in\right.$ $N\} \cup\left\{\left(\chi^{N}, 1\right)\right\}$ is a representative set for $D_{z}=D_{p}$. Using theorem 1.18.A and the previous table we obtain our results for $\mathbf{1 C}_{0}^{N}$ again, as described in corollary 3.8. Applying theorem 1.18.B we obtain

Theorem 3.10 The cone $\mathrm{1C}_{+}^{N}$ is generated by the extreme directions

$\operatorname{mix}_{\Omega^{\prime}}\left(u^{\{i\}}\right), i \in N$, and $\operatorname{mix}_{n^{\prime}}\left(w^{(N))}\right.$, with $\Omega^{\prime} \subseteq 2^{N}, \Omega \subseteq \Omega^{\prime}$.

The game $w^{N}=\operatorname{mix}_{2^{N}}\left(w^{N}\right)$ is non-simple, and, thus, $1 C_{+}^{N}$ possesses nonsimple extreme games.

\section{The clan games}

A game $v$ is called a clan game if there exists a non-empty coalition $C$, the clan, such that

1. $v \geq 0, b^{v} \geq 0$,

2. $v(S)=0$ for all $S \subseteq N, C \nsubseteq S$, and

3. $v(S) \leq v(N)-\sum_{j \in N \backslash S} b_{j}^{v}$ for all $\emptyset \neq S \subseteq N, C \subseteq S$.

Clan games are introduced and discussed in Potters, Poos and Muto [1989]. In their paper the class CIAN ${ }^{N, C}$ of clan games with respect to a clan $C \neq 0$ is characterized as a cone generated by its simple games. Here we will present a proof of this result with the help of the following linear mapping, which actually equals the mapping, defined in (3.3), on the coalitions containing the clan $C$ :

$$
f_{C}^{(x, \gamma)}(S)=\left\{\begin{array}{cl}
\sum_{j \in S} x_{j}-\gamma & \text { if } S \subseteq N, C \subseteq S, \\
0 & \text { otherwise. }
\end{array}\right.
$$

Of course, $f_{C}(x, \gamma)$ fulfils item 2 of the definition of a clan game, and one easily checks that item 3 is also valid. In fact, the items 2 and 3 can be replaced by

$$
v \leq f_{C}\left(b^{v}, \gamma^{v}\right)
$$


for non-negative games $v$. Observe that the games $v$ and $f_{C}\left(b^{v}, \gamma^{v}\right)$ are equal on the coalitions with at least $|N|-1$ players.

For the players $i$ outside the coalition $C$ the marginal contribution allocation of $f_{C}(x, \gamma)$ equals $f_{C}^{(x, y)}(N)-f_{C}^{(x, \gamma)}(N \backslash\{i\})=x_{i}$, and it equals $f_{C}^{(x, y)}(N)=\sum_{j \in N} x_{j}-\gamma$ for the players in $C$. Therefore, $f_{C}(x, \gamma)$ fulfils item 1 of the definition of a clan game if and only if $\sum_{j \in S} x_{j} \geq \gamma$ for all coalitions $S$ enclosing $C$, and $x_{i} \geq 0$ for all $i \in N \backslash C$. Notice that this is equivalent to

$$
\sum_{j \in C} x_{j} \geq \gamma \text {, and } x_{i} \geq 0 \text { for all } i \in N \backslash C \text {. }
$$

Let $D_{p}$ denote the set of elements $(x, \gamma)$, which fulfil the above restrictions. Then

$$
f_{C}(x, \gamma) \in \text { CLAN }^{N, C} \text { if and only if }(x, \gamma) \in D_{p} .
$$

Observe that for a clan game $v \in \operatorname{CLAN}^{N, C}$ we have $\left(b^{v}, \gamma^{v}\right) \in D_{p}$. For a non-negative game $v$ we in fact have

$$
v \in \mathbf{C L A N}^{N, C} \text { if and only if }\left(b^{v}, \gamma^{v}\right) \in D_{\mathrm{p}} \text { and } v \leq f_{C}\left(b^{v}, \gamma^{v}\right) \text {. }
$$

We conclude that the class CLAN ${ }^{N, C}$ equals $\mathbf{W}_{+}^{N}$ in theorem 1.17 with linear mapping $f=f_{C}$, the set $\Omega$ consisting of $N$ and the $(|N|-1)$-person coalitions, and with domain $D_{p}$ as described above.

Let us consider $D_{p}$ more closely. Observe that the kernel of $f_{C}$

$$
\begin{aligned}
D_{0} & =\left\{(x, \gamma): f_{C}(x, \gamma) \text { is the all-losing game }\right\} \\
& =\left\{(x, \gamma): x_{i}=0 \text { for } i \in N \backslash C \text { and } \sum_{j \in C} x_{j}=\gamma\right\}
\end{aligned}
$$

is included in $D_{p}$. Furthermore, for an element $(x, \gamma) \in D_{p}$ we have

$$
(x, \gamma)=\sum_{k \in N \backslash C} x_{k}\left(\chi^{\{k\}}, 0\right)+\left(\sum_{j \in C} x_{j}-\gamma\right)\left(\chi^{\{i\}}, 0\right)+\left(x^{\prime}, \gamma\right)
$$

with $i \in C$ arbitrary, and $x^{\prime} \in I R^{N}$ appropiately chosen such that the above equality holds. Since we must have $\sum_{j \in C} x_{j}^{\prime}=\gamma$, the element $\left(x^{\prime}, \gamma\right)$ is contained in $D_{0}$. Thus, an element $(x, \gamma) \in D_{p}$ is a non-negative weighted combination of the elements $\left(\chi^{\{i\}}, 0\right), i \in N$ of $D_{p}$ and an element of $D_{0}$.

Applying theorem 1.17 we conclude that $\mathbf{W}^{N}=\mathrm{CLAN}^{N, C}$ is generated by the games mix $\Omega^{\prime}\left(f_{C}\left(\chi^{(i)}, 0\right)\right), i \in N$, and $\Omega^{\prime} \subseteq 2^{N}$ such that it contains $N$ and $N \backslash\{j\}, j \in N$. If $i$ is a member of $C$ then the game $\operatorname{mix}_{\Omega^{\prime}}\left(f_{C}\left(\chi^{\{i\}}, 0\right)\right)$ equals $\operatorname{mix}_{\Omega^{\prime}}\left(u^{C}\right)$, and $\operatorname{mix}_{\Omega^{\prime}}\left(f_{C}\left(\chi^{\{i\}}, 0\right)\right)$ equals $\operatorname{mix}_{\Omega^{r}}\left(u^{C \cup\{i\}}\right)$ for $i \in N \backslash C$. These games are simple. Therefore, 
Theorem 3.11 The cone $\mathrm{CLAN}^{N, C}$ is generated by its simple games. Furthermore, these games are extreme in $\mathrm{CLAN}^{N, C}$.

Proof: We saw already that CLAN ${ }^{N, C}$ is generated by simple games. This proves the first assertion. According to lemma 1.20 we may conclude that these games are extreme.

\subsection{The class of semiconvex games}

For a semibalanced game $v$ the semibalancedness conditions,

$$
v(S) \leq \sum_{j \in S} b_{j}^{v} \quad \text { for } \emptyset \neq S \subseteq N
$$

may even hold if an arbitrary marginal contribution $b_{i}^{v}$ in the sum $\sum_{j \in S} b_{j}^{v}$ is replaced by the individual worth $v(\{i\})$. Observe that we interpret the marginal contribution of a player as a large pay-off to the player and the individual worth as a small pay-off. Inspired by these reasonings, the class of semiconvex games is defined by

$$
\begin{array}{r}
\mathbf{S C}^{N}=\left\{v \in \mathbf{G}^{N}: b^{v} \geq v \text { and } \sum_{j \in S \backslash\{i\}} b_{j}^{v}+v(\{i\}) \geq v(S)\right. \\
\text { for all } i \in N, \text { and } S \subseteq N, \text { with } i \in S\}
\end{array}
$$

(we assume in this section that the player set $N$ consists of at least 3 players). Semiconvex games were introduced in Driessen and Tijs [1985] as an adjunct to the study of the $\tau$-value concept. There it was also established that $\mathbf{S C}^{N}$ is a $\left(2^{|N|}-1\right)$-dimensional cone in $\mathbf{G}^{N}$ which includes the class of convex games. In fact, only some of the convexity conditions already imply the semiconvexity of a game. To show this, let $v$ fulfil

$$
v(N)+v(S \backslash\{i\}) \geq v(N \backslash\{i\})+v(S) \text { for all } i \in N, S \subseteq N, i \in S
$$

Notice that (3.5) is equivalent to

$$
b_{i}^{v} \geq v(S)-v(S \backslash\{i\}) \text { for all } i \in N, S \subseteq N, i \in S,
$$

and this expresses the condition that the marginal contribution of a player to a coalition is largest for the grand coalition (notice that in this case the marginal core of a game $v$ equals the set $M(v)$ of efficient allocations, mentioned on page 13). The semiconvexity condition $b^{v}(S \backslash\{i\})+v(\{i\}) \geq$ 
$v(S)$ for an arbitrary non-empty coalition $S=\left\{i_{1} i_{2}, \ldots, i_{|S|}\right\}$ now follows from the $|S|-1$ inequalities $b_{i_{2}}^{u} \geq v\left(\left\{i_{1}, i_{2}\right\}\right)-v(\{i\}), b_{i_{3}}^{v} \geq v\left(\left\{i_{1} i_{2}, i_{3}\right\}\right)-$ $v\left(\left\{i_{i} i_{2}\right\}\right), \ldots, b_{i_{|S|}}^{v} \geq v(S)-v\left(S \backslash\left\{i_{|S|}\right\}\right)$.

$\mathrm{SC}^{N}$ does not contain all games with a non-empty core. For instance, $u^{\{i\}}-1^{\{i\}}$ is not semiconvex. Although this game has a non-empty core and, thus, fulfils the semibalancedness inequalities these inequalities cannot be strengthened as is done above by replacing $b_{i}^{(i)-1^{\{i\}}}=\chi_{i}^{\{i\}}=1$ by $\left(u^{\{i\}}-1^{\{i\}}\right)(\{i\})=0$ since

$$
\sum_{j \in S \backslash\{i\}} b_{j}^{u^{(i)}-\mathbf{1}^{\{i\}}}+\left(u^{\{i\}}-\mathbb{1}^{\{i\}}\right)(\{i\})=0<1=\left(u^{\{i\}}-\mathbb{1}^{\{i\}}\right)(S)
$$

for a non-empty coalition $S \neq\{i\}$, with $i \in S$.

Notice that the maximal remainder allocation of a game $v$ in $\mathbf{S C}^{N}$, defined by $a^{v}=b^{v}+\left(\max _{S \ni i}\left(v(S)-\sum_{j \in S} b_{j}^{v}\right)\right)_{i \in N}$, equals the allocation $(v(\{i\}))_{i \in N}$. Actually, the semiconvexity property of a game is equivalent to

$$
a_{i}^{v}=v(\{i\}) \text { for each player } i \text {. }
$$

Thus, the core cover of a semiconvex game is the intersection of the imputation set and the marginal core.

The airport cost savings games

Let us show the semiconvexity property for the airport cost savings games $w^{S}$, with $S \subseteq N,|S| \geq 2$. Recall that $w^{S}$ is 0 -normalized and $b^{w^{S}}$ equals $\chi^{S}$. Then, for arbitrary player $i$,

$$
\begin{aligned}
\sum_{j \in S \backslash\{i\}} b_{j}^{w^{T}}+w^{T}(\{i\}) & =0=w^{T}(S) \text { for } S \subseteq N, \text { with } S \cap T=\emptyset, \\
& \geq|S \cap T|-1=w^{T}(S) \text { for } S \subseteq N, S \cap T \neq \emptyset .
\end{aligned}
$$

This proves the semiconvexity of $w^{T}$. In fact these games are convex as one can easily check.

All airport cost savings games with respect to non-negative cost allocations are contained in the cone $\mathrm{AC}^{N}$ generated by the games $w^{T}, T \subseteq$ $N,|T| \geq 2$ (theorem 2.16). Since the latter games are semiconvex and $\mathrm{SC}^{\bar{N}}$ is a cone, the mentioned airport cost savings games are contained in $\mathbf{S C}^{N}$.

\subsubsection{A decomposition of the cone of semiconvex games}

Our main goal here is the decomposition the class of semiconvex games into an algebraic sum of cones of games for which finite generating sets are 
available. The union of these sets thus forms a generating set for $\mathbf{S C}^{N}$, In particular, we will decompose $\mathbf{S C}^{N}$ into three cones. One is the cone $\mathbf{A C}{ }^{N}$. Notice that $\mathbf{A C}{ }^{N}$ is generated by convex games and, thus, consists of convex games. In subsection 1.2.3 we mentioned that the core of a convex game equals the Weber set. The Weber set of a game is always non-empty containing all core allocations and in this sense the core of a convex game is as large as possible. One of the two other cones is $\mathbf{H}^{N}$ consisting of games $v$ with $(v(\{i\}))_{i \in N}$ as the only core allocation. $\mathbf{H}^{N}$ has been discussed in subsection 2.2.1. The remaining cone is generated by the game $-\hat{w}$ which has an empty core (see also section 3.2). This decomposition is also discussed in Derks and Driessen [1990].

\section{Theorem 3.12 $\mathbf{S C}^{N}=\mathbf{H}^{N}+\mathbf{A C}^{N}+\operatorname{Cone}(\{-\hat{w}\})$.}

Proof: The semiconvexity of the game $-\hat{w}$ follows from its semibalancedness and $b^{\hat{w}}=0=(\hat{w}(\{i\}))_{i \in N}=0$.

Observe that the additive games are semiconvex. Therefore, $\mathrm{SC}^{N}=$ $\mathbf{A}^{N}+\mathbf{S C}_{0}^{N}$ according to lemma 1.19. Let $v \in \mathbf{S C}_{0}^{N}$. The semibalancedness of $v$ yields $b^{v} \geq 0$. Therefore, the airport cost savings game $w_{b v}$ is an element of the cone $\mathbf{A C}^{N}$ according to theorem 2.16. Consider the scalar

$$
\alpha=\sum_{j \in N} b_{j}^{v}-v(N)-\max _{j \in N} b_{j}^{v}
$$

The semiconvexity of $v$ yields that $\alpha \geq 0$. Thus, $-\alpha \hat{w} \in \operatorname{Cone}(\{-\hat{w}\})$. With respect to the game $w=v-w_{b^{v}}+\alpha \hat{w}$ we assert that $w$ is non-positive, with $w(N)=0$. Indeed,

$$
\begin{aligned}
w(N) & =v(N)-\sum_{j \in N} b_{j}^{v}+\max _{j \in N} b_{j}^{v}+\alpha=0, \\
w(N \backslash\{i\}) & =v(N \backslash\{i\})-\sum_{j \in N \backslash\{i\}} b_{j}^{v}+\max _{j \in N \backslash\{i\}} b_{j}^{v}+\alpha \\
& =\max _{j \in N \backslash\{i\}} b_{j}^{\nu}-\max _{j \in N} b_{j}^{v} \leq 0 \text { for all } i \in N, \text { and } \\
w(S) & =v(S)-\sum_{j \in S} b_{j}^{v}+\max _{j \in S} b_{j}^{v} \\
& \leq 0 \text { for each } S \subset N \text { with }|S| \leq|N|-2,
\end{aligned}
$$

where the last inequality results from the the 0 -normalizedness and semiconvexity of $v$. Hence, $w(S) \leq 0$ for all $S \subseteq N$. Together with $u(N)=0$ and $w(\{i\})=v(\{i\})=0$ for all $i \in N$, this implies that the zero allocation 
$(w(\{i\}))_{i \in N}$ is a core allocation of $w$ and, therefore, $w \in \mathbf{H}^{N}$. We conclude that $v=w+w_{b}-\alpha \hat{w} \in \mathbf{H}^{N}+\mathbf{A C}^{N}+\operatorname{Cone}(\{-\hat{w}\})$. Using $\mathbf{A}^{N} \subset \mathbf{H}^{N}$ we obtain $\mathbf{C}^{N}=\mathbf{A}^{N}+\mathbf{C}_{0}^{N} \subseteq \mathbf{H}^{N}+\mathbf{A C}^{N}+\operatorname{Cone}(\{-\hat{w}\})$.

The reverse inclusion follows from $\mathbf{A C}^{N} \subset \mathbf{S C}^{N}, \mathbf{H}^{N} \subset \mathbf{S C}^{N},-\hat{w} \in \mathbf{S C}^{N}$ and the fact that $\mathbf{S C}^{N}$ is a cone.

\section{A generating set}

By its definition, the cone $\mathrm{AC}^{N}$ is generated by the set $\left\{w^{s}: S \subseteq N\right.$, $|S| \geq 3\}$. A generating set for the cone $\mathbf{H}^{N}$ is the set $\left\{u^{\{i\}}: i \in N\right\} \cup\left\{-u^{\{i\}}\right.$ : $i \in N\} \cup\left\{-\mathbb{1}^{S}: S \subset N,|S| \geq 2\right\}$ (see theorem 2.3).

Corollary 3.13 A generating set for the class $\mathrm{SC}^{N}$ is formed by the $2^{|N|+1}$ 2 games $u^{\{i\}}$ and $-u^{\{i\}}$, with $i \in N,-\mathbb{1}^{S}$, with $S \subset N,|S| \geq 2$, w $w^{S}$, with $S \subseteq N,|S| \geq 2$, and $-\hat{w}$.

We have shown that the 0 -normalization, $u^{\{i\}}-\mathbb{1}^{\{i\}}$, of $u^{\{i\}}$ is not semiconvex. Since $u^{\{i\}} \in \mathbf{S C}^{N}$ we must therefore have $-\mathbb{1}^{\{i\}} \notin \mathbf{S C}^{N}$. Notice that this fully agrees with the observation that $-\mathbb{1}^{\{i\}} \in \mathbf{S C}^{N}$ for all $i \in \mathbf{S C}^{N}$ would imply that the whole generating set of $\mathrm{C}^{N}$, presented in theorem 2.1, is a subset of $\mathbf{S C}^{N}$ yielding $\mathbf{C}^{N} \subseteq \mathbf{S C}^{N}$, which is impossible.

The following inclusion relations are immediate. There are no other inclusions between the mentioned sets of games except of course the inclusions which follow from the listed ones ( $\mathrm{CV}^{N}$ denotes the class of convex games).

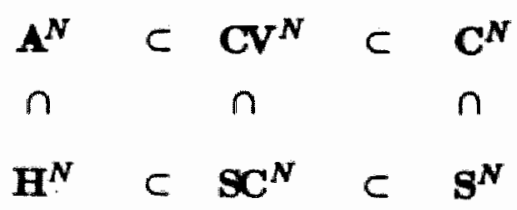

See also subsection 3.6.1.

\section{The 0-normalized semiconvex games}

Except for the additive games the generating games in the above corollary are 0-normalized and in fact they form a representative set of extreme directions of $\mathbf{S C}_{0}^{N}$ :

Theorem 3.14 The $2^{|N|+1}-2|N|-2$ games $-1^{S}$, with $S \subset N,|S| \geq 2, w^{S}$, with $S \subseteq N,|S| \geq 2$, and $-\hat{w}$ form a generating set of $\mathbf{S C}_{0}^{N}$. Furthermore, they are extreme.

Proof: Observe that a game in $\mathbf{A C}^{N}$ is 0-normalized, yielding $\mathbf{A C}^{N} \subseteq$ $\mathbf{S C}_{0}^{N}$. Also, $-\hat{\boldsymbol{w}} \in \mathbf{S C}_{0}^{N}$. We, thus, have $\mathbf{H}_{0}^{N}+\mathbf{A C}^{N}+\operatorname{Cone}(\{-\hat{w}\}) \subseteq$ 
$\mathbf{S C}_{0}^{N}$. Consider the decomposition of a 0 -normalized semiconvex game $v$ as $w+w_{b^{v}}-\alpha \hat{w}$ in the proof of theorem 3.12. The 0-normalizedness of the games $v, w_{b}$ and $-\alpha \hat{w}$ implies the 0 -normalizedness of $w \in \mathbf{H}^{N}$ and, thus, $\mathbf{H}_{0}^{N}+\mathbf{A C}^{N}+\operatorname{Cone}(\{-\hat{w}\}) \supseteq \mathbf{S C}_{0}^{N}$. In theorem 2.3 a generating set for $\mathbf{H}_{0}^{N}$ is presented. Using this result the set $W$ of games mentioned in the theorem is a generating set of $\mathbf{S C}_{0}^{N}$.

Let us prove the extremality of the games in $W$.

The extremality of $-\hat{w}$ and $-\mathbb{1}^{S}$, with $S \subset N, 2 \leq|S| \leq|N|-2$, follow from the fact that these games are extreme in the larger class of 0 -normalized semibalanced games (theorem 3.3).

The extremality of the game $-1^{N \backslash\{i\}}$, with $i \in N$, follows from the observation that in case $-1^{N \backslash\{i\}}$ equals a non-negative combination, say $\sum_{w \in W} \alpha^{w} w$, of elements of $W$, then

$$
\begin{aligned}
\chi^{\{i\}} & =b^{-\mathbf{1}^{N \backslash\{i\}}}=\sum_{w \in W} \alpha^{w} b^{w} \\
& =\sum_{j \in N} \alpha^{-\mathbf{1}^{N \backslash\{j\}}} \chi^{\{j\}}+\sum_{S \subseteq N,|S| \geq 2} \alpha^{w^{s}} \chi^{S},
\end{aligned}
$$

and this equality only holds if $\alpha^{-1^{N \backslash\{i)}}=1, \alpha^{-1^{N \backslash(j)}}=0$ for all $j \in N \backslash\{i\}$, and $\alpha^{w^{S}}=0$ for each coalition $S$ with $|S| \geq 2$. Then also $\alpha^{-1 s}=0$ for each $S \subset N$ with $2 \leq|S| \leq|N|-2$. Therefore, - $\mathbf{1}^{N \backslash(i)} \notin \operatorname{Cone}\left(W \backslash\left\{-\mathbf{1}^{N \backslash\{i\}}\right\}\right)$, implying its extremality.

Now let $w^{T}$ be a non-negative combination, say $\sum_{w \in W} \alpha^{w} w$, of elements of $W$, with $T \subseteq N,|T| \geq 2$, arbitrary. From

$$
\begin{aligned}
\chi^{T} & =b^{w^{T}}=\sum_{w \in W} \alpha^{w} b^{w} \\
& =\sum_{j \in N} \alpha^{-\mathbf{1}^{N \backslash(j)}} \chi^{\{j\}}+\sum_{S \subseteq N_{0}|S| \geq 2} \alpha^{w^{s}} \chi^{S}
\end{aligned}
$$

it follows that $\alpha^{w}=0$ for the games $w=-\mathbb{1}^{N \backslash\{j\}}$, with $j \in N \backslash T$, and $w=w^{S}$, for coalitions $S,|S| \geq 2$, such that $S \backslash T$ is non-empty. Then

$$
1=\chi_{i}^{T}=\alpha^{-\mathbf{1}^{N \backslash\{i}}+\sum_{S \subseteq T,|S| \geq 2, i \in S} \alpha^{w^{s}}, \quad \text { for each } i \in T \text {. }
$$

Adding the $|T|$ equalities of (3.8) we obtain

$$
|T|=\sum_{j \in T} \alpha^{-\mathbf{1}^{M \backslash(j)}}+\sum_{S \subseteq T,|S| \geq 2} \alpha^{\omega^{S}}|S| .
$$


Furthermore,

$$
\begin{aligned}
|T|-1 & =w^{T}(N)=\sum_{S \subseteq T,|S| \geq 2} \alpha^{w^{s}}(|S|-1)-\alpha^{-\hat{w}} \\
& =\sum_{S \subseteq T,|S| \geq 2} \alpha^{w^{S}}|S|-\sum_{S \subseteq T,|S| \geq 2} \alpha^{w^{s}}-\alpha^{-\hat{w}} .
\end{aligned}
$$

Combining (3.9) and (3.10) we obtain

$$
\sum_{j \in T} \alpha^{-\mathbf{1}^{N \backslash(j)}}+\sum_{S \subseteq T,|S| \geq 2} \alpha^{w^{S}}+\alpha^{-\hat{\boldsymbol{w}}}=1 .
$$

In combination with (3.8) this is only possible if $\alpha^{-\mathbf{1}^{N \backslash(j)}}=0$, for $j \in T$, $\alpha^{w^{T}}=1, \alpha^{w^{s}}=0$ for $S \subset T,|S| \geq 2$, and $\alpha^{-\hat{w}}=0$. It follows now that $\alpha^{-r^{s}}=0$ for all $S \subseteq N$, with $|S| \geq 2$, and we conclude that the game $w^{T}$ must be extreme.

The extremality of the game $-\hat{w}$ in $\mathbf{S C}_{0}^{N}$ also follows from the fact that it has an empty core, and $W \backslash\{\hat{w}\}$ is a subset of the cone of games with a non-empty core.

The game $w^{\mathcal{S}}$ as an extreme convex game

The extreme games of $\mathrm{SC}_{0}^{N}$ which are convex are extreme in the class $\mathbf{C V}_{0}^{N}$ of 0 -normalized convex games. according to the fact that this class is contained in $\mathbf{S C}_{0}^{N}$ as a subset. Hence,

Corollary 3.15 The games $w^{S}$, with $S \subseteq N,|S| \geq 2$, are extreme in the class of the O-normalized convex games.

Notice that lemma 1.19 yields the decomposition of the cone $\mathrm{CV}_{+}^{N}$ of nonnegative convex games as the algebraic sum $\mathbf{A}_{+}^{N}+\mathbf{C V}_{0}^{N}$. The extreme games of $\mathbf{C V}_{+}^{N}$ have been characterized in Rosenmüller and Weidner [1974]. There a unique representation is given for a non-negative convex game $v$ as a coalition-wise maximum of games $w^{(q, Q)}$ with $q \in I R_{+}^{N}$ and $Q \in I R_{+}$, defined by

$$
w^{(q, Q)}(S)=\left(\sum_{j \in S} q_{j}-Q\right)_{+} \quad \text { for each } S \subseteq N \text { non-empty }
$$

(where $(\alpha)_{+}$is defined to be $\max (\alpha, 0)$ ). Rosenmüller and Weidner showed that the extremality of a non-negative convex game in $\mathrm{CV}_{+}^{N}$ is equivalent to a particular property of the corresponding representation. 
Games of type (3.11) are also discussed in O'Neill [1982] where they are called bankruptcy games. These games arise from a bankruptcy situation in which there is an estate of size $E \in I R_{+}$, which has to be divided among a group $N$ of heirs. Each heir $i \in N$ is assumed to claim a non-negative portion, say $d_{i} \geq 0$, of the estate $E$. We assume that the sum of the claims $D=\sum_{j \in N} d_{j}$ exceeds the estate $E$. Each coalition $S$ can get at least the portion

$$
\left(E-\sum_{j \in N \backslash S} d_{j}\right)_{+}=\left(\sum_{j \in S} d_{j}-(E-D)\right)_{+}
$$

which equals the worth of $S$ in the game $w^{(d, E-D)}(S)$; i.e., $w^{(d, E-D)}$ expresses the minimal profit of the coalitions. If every claim is majorized by $E-D$, then the corresponding bankruptcy game is 0-normalized.

Now notice that the airport cost savings game $w^{S}, S \subseteq N,|S| \geq 2$, is a bankruptcy game, with estate $E=|S|-1$ and claims 1 for each player in $S$ and 0 otherwise, i.e., $d=\chi^{S}$.

\subsection{Cones of quasi-balanced games}

The core cover $Q \operatorname{Core}(v)=\left\{x \in I R^{N}: a^{u} \leq x \leq b^{v}\right.$ and $\left.\sum_{j \in N} x_{j}=v(N)\right\}$ of a quasi-balanced game $v$ is by definition non-empty and contains all core allocations (theorem 1.9). The class $\mathbf{C}^{N}$ of games with a non-empty core is therefore a subset of $\mathrm{Q}^{N}$. In case $\sum_{j \in N} b_{j}^{v}$ equals $v(N)$, the marginal contribution allocation $b^{v}$ is a core allocation, since $v$ is semibalanced. This situation occurs for example if $a^{v}=b^{v}$ holds. If these allocations are equal up to one entry then the maximal remainder allocation $a^{v}$ is a core allocation.

Lemma 3.16 Let $v$ be a quasi-balanced game and let coalition $S$ be the set of all players $i$ with $a_{i}^{v}<b_{i}^{v}$. If $|S|<2$ or $\sum_{j \in N} b_{j}^{v}=v(N)$ then $v \in \mathbf{C}^{N}$.

Proof: If $|S|=0$ then $a^{v}=b^{v}$, implying $v(N)=\sum_{j \in N} b_{j}^{v}$, i.e., $b^{v}$ is an efficient allocation and, thus, a core allocation.

Now suppose $|S|=1$ and let $i$ be the only player in $S$. Then $\sum_{j \in N \backslash\{i\}} b_{j}^{u}=$ $\sum_{j \in N \backslash\{i\}} a_{j}^{v}$. Thus,

$$
b_{i}^{\nu}-a_{i}^{v} \leq \sum_{j \in N} b_{j}^{v}-v(N) \leq \sum_{j \in N} b_{j}^{\nu}-\sum_{j \in N} a_{j}^{v}=b_{i}^{v}-a_{i}^{\nu},
$$

where the first inequality directly follows from the definition of $a^{v}$. We conclude that $v(N)$ equals $\sum_{j \in N} a_{j}^{v}$. Moreover, for each coalition $T \subseteq N \backslash\{i\}$ we have $v(T) \leq \sum_{j \in T} b_{j}^{v}=\sum_{j \in T} a_{j}^{v}$, and for $T \subseteq N, i \in T$, we have 
$a_{i}^{v} \geq v(T)-\sum_{j \in T \backslash\{i\}} b_{j}^{v}=v(T)-\sum_{j \in T \backslash\{i\}} a_{j}^{v}$. Therefore, the maximal remainder vector majorizes $v$. Together with the efficiency of $a^{v}$ we conclude that $a^{v}$ is a core allocation of $v$.

\subsubsection{The cone of 0-normalized quasi-balanced games}

The set $\mathbf{Q}_{0}^{N}=\mathbf{Q}^{N} \cap \mathbf{G}_{0}^{N}$ of the 0-normalized quasi-balanced games is a polyhedral cone, contained in the pointed cone of 0 -normalized semibalanced games as a subset, and, therefore, it is also pointed. Consequently, there is a finite generating set of extreme directions for $Q_{0}^{N}$ and our main result in this section is the construction of such generating set.

\section{Preliminaries}

The airport cost savings games $w^{S}$, with $S \subseteq N,|S| \geq 2$, and $-\hat{w}$ are semibalanced and 0-normalized. The games $v^{S}$ defined by

$$
v^{S}=w^{S}-(|S|-1) \hat{w} \quad \text { for } S \subseteq N,|S| \geq 2,
$$

i.e.,

$$
v^{S}(T)=\left\{\begin{array}{cl}
0 & \text { if } T=N \text { or } T=N \backslash\{i\} \text { for an } i \notin S, \\
-1 & \text { if } T=N \backslash\{i\} \text { for an } i \in S, \\
|S \cap T|-1 & \text { if } T \subset N,|T| \leq|N|-2, \text { and } S \cap T \neq \emptyset \\
0 & \text { otherwise, }
\end{array}\right.
$$

are therefore semibalanced and 0 -normalized also. Notice that if there are at most three players then these games equal the all-losing game or $-\mathbf{1}^{N \backslash\{i\}}$, $i \in N$.

The marginal contribution allocation of $v^{s}$ equals the sum of $b^{w^{s}}=\chi^{s}$ and $(|S|-1) b^{-w}=0$; hence, $b^{v^{s}}=\chi^{S}$. For each $i \in S$ we thus have

$$
\begin{aligned}
a_{i}^{v^{s}} & =\chi_{i}^{S}+\max _{T \ni i}\left(\left(w^{S}-(|S|-1) \hat{w}\right)(T)-|S \cap T|\right) \\
& =1+\max _{T \ni i}(|S \cap T|-1-(|S|-1) \hat{w}(T)-|S \cap T|) \\
& =1+\max _{T \ni i}(-(|S|-1) \hat{w}(T)-1) \\
& =0,
\end{aligned}
$$

and for each player $i$ outside of $S$ we have $v^{s}(\{i\})=0=b_{i}^{v^{s}}$, implying $a_{i}^{v^{s}} \geq b_{i}^{v^{s}}$; now equality holds since $v^{s}$ is sb. Thus, $v^{s}$ fulfils

$$
a^{v}=0, v(N)=0 \text { and } b^{v}=\chi^{S} \text {. }
$$


We therefore conclude that $v^{S}$ is quasi-balanced, for $S \subseteq N,|S| \geq 2$. The game $v^{S}$ has the property that it majorizes each game which fulfils ( 3.12 ). To show this let $v$ be an arbitrary game for which (3.12) holds; then $v$ obviously equals $v^{S}$ for coalitions with at least $|N|-1$ players, and for a coalition $T$ with $|T| \leq|N|-2$, we have

$$
v(T) \leq\left(a_{i}^{\nu}-b_{i}^{v}\right)+\sum_{j \in T} b_{j}^{v}=-\chi_{i}^{S}+\chi^{S}(T)=|S \cap T|-\chi_{i}^{S}=v^{S}(T) .
$$

\section{A 'Blicing' result}

The games $v^{S}, S \subseteq N,|S| \geq 2$, are shown to be extreme in $\mathrm{Q}_{0}^{N}$. Although there is a striking similarity with the extreme games of $\mathbf{S}_{+}^{N}$ (see theorem 3.5) a similar extremality proof is not (yet) available.

The following result will play a central role.

Lemma 3.17 Let $v$ be a $q b$ game and let $S$ denote the set of players $i \in N$ for which $a_{i}^{v}<b_{i}^{v}$. If $|S| \geq 2$ and $v(N)<b^{v}(N)$ then there is an $\alpha>0$ such that $v-\alpha v^{S} \in \mathbf{Q}^{N}$.

Proof: Choose $\alpha>0$ such that

$$
v(N) \leq \sum_{j \in N} b_{j}^{v}-\alpha|S|
$$

and

$$
a_{i}^{v} \leq b_{i}^{v}-\alpha \text { for all } i \in S,
$$

and consider the game $v^{1}=v-\alpha v^{S}$. This game may be seen as constructed from $v$ by increasing the worths of the coalitions $N \backslash\{i\}$, thus decreasing $b_{i}^{v}$, by an amount $\alpha$ for those players $i$ for whom there is a positive gap between the vectors $a^{v}$ and $b^{v}$, i.e., the players in $S$. Furthermore, the worths of the other coalitions $T$, with $|T| \leq|N|-2$, are decreased by a maximal amount without losing the quasi-balancedness property (as will be shown).

Let us first show the semibalancedness of $v^{1}$. The marginal contribution allocation $b^{v^{1}}$ equals $b^{v}-\alpha b^{v^{s}}=b^{v}-\alpha \chi^{s}$. From

$$
\begin{aligned}
v^{1}(N) & =v(N)-\alpha v^{S}(N)=v(N) \\
& \leq \sum_{j \in N} b_{j}^{\psi}-\alpha|S| \\
& =\sum_{j \in N} b_{j}^{v^{1}}
\end{aligned}
$$


and (2.2) we have, for each coalition $T$ with at least $|N|-1$ players,

$$
v^{1}(T)-\sum_{j \in T} b_{j}^{v^{1}}=v^{1}(N)-\sum_{j \in N} b_{j}^{v^{1}} \leq 0 .
$$

For the other coalitions $T$ with $T \cap S \neq \emptyset$, say $i \in T \cap S$, we have

$$
\begin{aligned}
v^{1}(T)-\sum_{j \in T} b_{j}^{v^{1}} & =v(T)-\alpha v^{S}(T)-\sum_{j \in T} b_{j}^{v}+\alpha|S \cap T| \\
& =v(T)-\sum_{j \in T} b_{j}^{v}+\alpha \\
& \leq a_{i}^{v}-b_{i}^{v}+\alpha \leq 0,
\end{aligned}
$$

and for the remaining non-empty coalitions $T$, say $i \in T$, we have

$$
\begin{aligned}
v^{1}(T)-\sum_{j \in T} b_{j}^{v^{1}} & =v(T)-\sum_{j \in T} b_{j}^{v} \\
& \leq a_{i}^{v}-b_{i}^{v} \leq 0 .
\end{aligned}
$$

Thus $v^{1}(T)-\sum_{j \in T} b_{j}^{v^{1}} \leq 0$ for all $T \subseteq N$ and, therefore, $v^{1}$ is semibalanced.

The semibalancedness of $v^{1}$ implies $a^{v^{1}} \leq b^{v^{1}}$ according to lemma 1.8. For the players $i$ in $S$ we derive a more useful majorization for $a_{i}^{v^{1}}$ in order to show the quasi-balancedness property for $v^{1}$. Using (3.15) and (2.2) we have, for $i \in S$,

$$
\begin{aligned}
a_{i}^{v^{1}} & =b_{i}^{v^{1}}+\max _{T \ni i}\left(v^{1}(T)-\sum_{j \in T} b_{j}^{v^{1}}\right) \\
& =b_{i}^{v^{1}}+\max \left(v^{1}(N)-\sum_{j \in N} b_{j}^{v^{1}}, \max _{T \ni i,|T| \leq|N|-2}\left(v^{1}(T)-\sum_{j \in T} b_{j}^{v^{1}}\right)\right) \\
& \leq b_{i}^{v^{1}}+\max \left(v(N)-\sum_{j \in N} b_{j}^{v}+\alpha|S|, a_{i}^{v}-b_{i}^{v}+\alpha\right)
\end{aligned}
$$

The scalar $\alpha$ is chosen such that $v(N)-\sum_{j \in N} b_{j}^{v}+\alpha|S| \leq 0$ and $a_{i}^{v}-b_{i}^{v}+\alpha \leq 0$ for each $i \in S$. Let us now consider the following two cases:

- there is a player $i$ of $S$ with $v(N)-\sum_{j \in N} b_{j}^{v}+\alpha|S| \geq a_{i}^{v}-b_{i}^{v}+\alpha$. Then (3.16) implies $a_{i}^{v^{1}} \leq b_{i}^{v^{1}}+v(N)-\sum_{j \in N} b_{j}^{v}+\alpha|S|$ and, thus,

$$
\begin{aligned}
\sum_{j \in N} a_{j}^{v^{1}} & \leq \sum_{j \in N \backslash\{i\}} b_{j}^{v^{1}}+b_{i}^{v^{1}}+v(N)-\sum_{j \in N} b_{j}^{v}+\alpha|S| \\
& =\sum_{j \in N} b_{j}^{v}-\alpha|S|+v(N)-\sum_{j \in N} b_{j}^{v}+\alpha|S| \\
& =v(N)=v^{1}(N) .
\end{aligned}
$$


- $v(N)-\sum_{j \in N} b_{j}^{v}+\alpha|S| \leq a_{i}^{v}-b_{i}^{\natural}+\alpha$ for all $i \in S$. Then, for $i \in S$ we must have $a_{i}^{v^{1}} \leq b_{i}^{v^{1}}+a_{i}^{v}-b_{i}^{v}+\alpha=a_{i}^{y}$ according to (3.16). Using $b_{i}^{v^{1}}=b_{i}^{v}=a_{i}^{v}$ for $i \notin S$, we obtain

$$
\begin{aligned}
\sum_{j \in N} a_{j}^{v^{1}} & =\sum_{j \in S} a_{j}^{v^{1}}+\sum_{j \in N \backslash S} a_{j}^{v^{1}} \\
& \leq \sum_{j \in S} a_{j}^{v}+\sum_{j \in N \backslash S} b_{j}^{v^{1}} \\
& =\sum_{j \in N} a_{j}^{v^{1}} \leq v(N)=v^{1}(N) .
\end{aligned}
$$

We conclude that $\sum_{j \in N} a_{j}^{v^{1}} \leq v^{1}(N)$. Together with $a^{v^{1}} \leq b^{v^{1}}$ we derive the quasi-balancedness of $v^{1}=v-\alpha v^{S}$.

The extreme directions of $Q_{0}^{N}$

We are now able to formulate a generating set for $Q_{0}^{N}$ and prove that its elements are extreme directions.

Theorem 3.18 The cone $Q_{0}^{N}$ equals the algebraic sum of the cone $\mathrm{C}_{0}^{N}$ of 0 -normalized games with a non-empty core and the cone generated by the games $v^{S}, S \subseteq N,|S| \geq 2$. The extreme directions are the games $u^{\{i\}}-\mathbb{1}^{\{i\}}$, $i \in N,-\mathbf{1}^{S}, S \subset N,|S| \geq 2$, and $v^{S}, S \subseteq N,|S| \geq 2$, up to a positive multiplication factor.

Proof: Let $W$ denote the set of games, mentioned in the theorem. Let $v$ be a 0-normalized quasi-balanced game. Observe that either lemma 3.16 or lemma 3.17 can be applied to $v$. Thus, either $v \in \mathbf{C}_{0}^{N}=\operatorname{Cone}\left(\left\{u^{\{i\}}-1^{\{i\}}\right.\right.$ : $\left.i \in N\} \cup\left\{-1^{S}:|S| \subset N,|S| \geq 2\right\}\right)$ according to theorem 2.5, or there is a coalition $S \subseteq N,|S| \geq 2$, and $\alpha>0$ such that the 0-normalized game $v-\alpha v^{S}$ is quasi-balanced. Therefore, whenever $v \in \mathbf{Q}_{0}^{N}$ there is a game $w \in W$ and $\alpha>0$ such that $v-\alpha w \in \mathbf{Q}_{0}^{N}$. The pointed cone property of $\mathrm{Q}_{0}^{N}$ and theorem 1.15 now imply $\mathrm{Q}_{0}^{N}=\operatorname{Cone}(W)$.

It remains to prove the extremality of the games in $W$. Note that $Q_{0}^{N}$ is a subset of $S_{0}^{N}$. Extremality of the games $u^{\{i\}}-1^{\{i\}}, i \in N$, and $-1^{S}$, $|S| \subset N,|S| \geq 2$, now follows from the fact that they are extreme in $\mathbf{S}_{0}^{N}$ (theorem 3.3).

Now consider the game $v^{S}$ as a non-negative weighted sum $\sum_{w \in W} \alpha^{w} w$, for an arbitrary coalition $S,|S| \geq 2$. Without loss of generality assume that 
$\alpha^{v^{s}}=0$. In case $N$ does not consist of more than three players the game $v^{S}$ equals one of the games in $W$ considered before. We, therefore, assume $|N| \geq 4$.

Since $w(N) \geq 0$ for all $w \in W$ and $v^{S}(N)=0$ we must have $\alpha^{w}=0$ for those games $w \in W$ with $w(N)>0$. Thus, $\alpha^{u^{(i)}-\mathbf{1}^{\{i\}}}=0, i \in N$. We conclude that $v^{S}$ is majorized by $\sum_{T \subseteq N,|T| \geq 2} \alpha^{v^{T}} v^{T}$, implying that for each two players $i$ and $j$ in $S$ we must have

$$
1=v^{S}(\{i, j\}) \leq \sum_{T \subseteq N,\{i, j\} \subseteq T} \alpha^{v^{T}} .
$$

The marginal contribution allocation of a game in $W$ is non-negative, implying that the marginal contribution allocation $\chi^{S}$ of $v^{S}$ majorizes the $\operatorname{sum} \sum_{T \subseteq N_{v}|T| \geq 2} \alpha^{v^{T}} b^{v^{T}}$, i.e.,

$$
1=b_{i}^{v^{s}} \geq \sum_{T \subseteq N, i \in T_{i}|T| \geq 2} \alpha^{v^{T}}, \text { for each } i \in S,
$$

and

$$
0=b_{i}^{v^{s}} \geq \sum_{T \subseteq N, i \notin T,|T| \geq 2} \alpha^{v^{T}}, \text { for each } i \notin S
$$

From (3.19) we conclude that $\alpha^{v^{T}}=0$ for coalitions $T,|T| \geq 2$, containing players outside $S$. Furthermore, the inequalities (3.17) and (3.18) only hold if we have equality for both, implying that the scalars $\alpha^{v^{T}}$ equal zero for $T \subset S$. We already observed that $\alpha^{v^{T}}=0$ for $T \subseteq N, T \backslash S \neq \emptyset$, and we assumed $\alpha^{v}=0$. Therefore, the only possible games $w$ left in $W$ with positive scalar $\alpha^{w}$ are the non-positive games $-1^{S}, S \subset N,|S| \geq 2$. This is contradictory to the observation that some of the two-person coalitions have positive worths in $v^{s}$. We conclude that $v^{S}$ is extreme in Cone $(W)=\mathbf{Q}_{0}^{N}$.

\subsubsection{The cone of quasi-balanced games}

Using the decomposition of $Q^{N}$ into the algebraic sum of the cones $A^{N}$ and $Q_{0}^{N}$ (see lemma 1.19) the following theorem is a direct consequence of theorem 3.18.

Theorem 3.19 The class $\mathrm{Q}^{N}$ of quasi-balanced games is generated by the $2^{|N|+1}+|N|-3$ games $u^{\{i\}},-u^{\{i\}}, u^{\{i\}}-1^{\{i\}}$, with $i \in N,-\mathbb{1}^{S}, S \subset N$, $|S| \geq 2$, and $v^{S}$, with $S \subseteq N,|S| \geq 2$. 
The mentioned games, except $u^{S}, S \subseteq N,|S| \geq 2$, form a generating set of the cone of games with a non-empty core according to theorem 2.1. Notice also that the only possible core allocation of $v^{S}$, with $|S| \geq 2$, is the zero allocation and this would imply that $v^{S}$ is non-positive, which occurs only for player sets $N$ with at most 3 players.

Corollary 3.20 $\mathrm{Q}^{N}=\mathrm{C}^{N}+\operatorname{Cone}\left(\left\{v^{S}: S \subseteq N,|S| \geq 2\right\}\right)$ and $\mathbf{Q}^{N}=\mathbf{C}^{N}$ if $N$ consists of at most three players.

The game $v^{S}$ has been defined for a coalition $S$ with more than one player. Allowing one-person coalitions in the definition of these games we would obtain zero games. We stated that $v^{S}$ is the maximum of games which fulfil (3.12). Now for $S=\{i\}$ it is easily checked that the quasi-balanced game $-\mathbb{1}^{N \backslash\{i\}}$ is maximal with respect to $(3.12)$, with $i \in N$. It is therefore a natural extension to define $v^{\{i\}}$ to be the game $-\mathbf{1}^{N \backslash\{i\}}, i \in N$.

Applying corollary 3.20 and theorem 2.5 we conclude that the class of quasi-balanced games is the algebraic sum of the cone generated by the games $v^{S}, \emptyset \neq S \subseteq N$, and the cone $D^{N}$ of games for which the marginal contribution allocation is a core allocation.

Corollary 3.21 $\mathbf{Q}^{N}=\mathbf{D}^{N}+\operatorname{Cone}\left(\left\{v^{S}: \emptyset \neq S \subseteq N\right\}\right)$.

\section{The ideally covered games}

First let us list the generating games in theorem 3.19, their maximal remainder allocation (a), marginal contribution allocation (b), and their values with respect to the solution schemes core, marginal core (MCore), core cover (QCore) and $\tau$-value $(\tau)$. In the table $i$ denotes a player and $S$ and $T$ are coalitions with $2 \leq|S| \leq|N|-2$ and $|T| \geq 2$ respectively.

\begin{tabular}{|c|c|c|c|c|c|c|}
\hline game & $a$ & $b$ & Core & MCore & QCore & $\tau$ \\
\hline$u^{\{i\}}$ & $\chi^{\{i\}}$ & $\chi^{\{i\}}$ & $\left\{\chi^{\{i\}}\right\}$ & $\left\{\chi^{\{i\}}\right\}$ & $\left\{\chi^{\{i\}}\right\}$ & $\chi^{\{i\}}$ \\
$-u^{\{i\}}$ & $-\chi^{\{i\}}$ & $-\chi^{\{i\}}$ & $\left\{-\chi^{\{i\}}\right\}$ & $\left\{-\chi^{\{i\}}\right\}$ & $\left\{-\chi^{\{i\}}\right\}$ & $-\chi^{\{i\}}$ \\
$u^{\{i\}}-\mathbf{1}\{i\}$ & $\chi^{\{i\}}$ & $\chi^{\{i\}}$ & $\left\{\chi^{\{i\}}\right\}$ & $\left\{\chi^{\{i\}}\right\}$ & $\left\{\chi^{\{i\}}\right\}$ & $\chi^{\{i\}}$ \\
$-\mathbf{1}^{N \backslash\{i\}}$ & 0 & $\chi^{\{i\}}$ & $\{0\}$ & $*$ & $\{0\}$ & 0 \\
$-\mathbf{1}^{s}$ & 0 & 0 & $\{0\}$ & $\{0\}$ & $\{0\}$ & 0 \\
$\boldsymbol{v}^{T}$ & 0 & $\chi^{T}$ & empty & $* *$ & $\{0\}$ & 0 \\
\hline
\end{tabular}


$*: \quad \operatorname{MCore}\left(-\mathbb{1}^{N \backslash\{j\}}\right)=\operatorname{Conv}\left(\left\{\chi^{\{i\}}-\chi^{\{j\}}: j \in N\right\}\right) ;$
$* * \quad \operatorname{MCore}\left(v^{T}\right)=\operatorname{Conv}\left(\left\{\chi^{T}-|T| \chi^{\{j\}}: j \in N\right\}\right)$.

Notice that the core cover of all generating games consists of only one allocation, which has to be equal to the $r$-value.

In the table all games except $v^{T}, T \subseteq N,|T| \geq 2$, have a non-empty core which equals the core cover; games with this property are called the ideally covered games. Also the airport cost savings games $w^{S}, S \subseteq N$, $|S| \geq 2$, are ideally covered. This follows from the fact that $\chi^{S}-\chi^{\{\bar{i}\}}$ are core allocations for $i \in S$, which are also the extreme elements of the core cover of $w^{S}:$ QCore $\left(w^{S}\right)=\left\{x \in I R^{N}: 0 \leq x \leq \chi^{S}, \sum_{j \in N} x_{j}=|S|-1\right\}$. However, the class $\mathrm{AC}^{N}$ generated by these games is not a subset of the class of ideally covered games, as the following example shows.

For an airport cost savings game $w^{y}$ we have $a^{w^{y}}=\left(w^{y}(\{i\})\right)_{i \in N}=0$ since $w^{\prime \prime}$ is semiconvex, and thus (3.7) on page 98 holds. Furthermore, the marginal contribution allocation of $w^{y}$ equals $y$ whenever there are two equal maximal coordinates. In this case we have

$$
\text { QCore }\left(w_{y}\right)=\left\{x \in I R^{N}: 0 \leq x \leq y \text { and } x(N)=y(N)-\max _{j \in N} y_{j}\right\} .
$$

Assume that $y$ is chosen such that all its coordinates are positive and there exist two distinct players $i$ and $j$ such that $y_{i}+y_{j}=\max _{k \in N} y_{k}$. Then the allocation $z=y-y_{i} \chi^{\{i\}}-y_{j} \chi^{\{j\}}$ is an element of QCore $\left(w_{y}\right)$. Furthermore, $z_{i}+z_{j}=0<\min \left(y_{i}, y_{j}\right)=w^{y}(\{i, j\})$ Therefore, $z$ cannot be a core allocation of $w^{\psi}$. We conclude that $w^{\nu}$, which is an element of $\mathbf{A C}{ }^{N}$, is not ideally covered.

\subsubsection{A decomposition procedure}

The proof of lemma 3.17 provides us with a procedure to represent a quasibalanced game as a non-negative weighted sum of garnes mentioned in theorem 3.19. For a game $v \in Q_{0}^{N}$ consider the set $S^{v}$ of players $i$ for which $a_{i}^{v}<b_{i}^{v}$. If either $\left|S^{v}\right|<2$ or $v(N)=\sum_{j \in N} b_{j}^{v}$ then $a^{v}$ or $b^{v}$ is a core allocation of $v$ and the decomposition in (2.3) will give us the required representation.

Let us now suppose that $\left|S^{v}\right| \geq 2$ and $v(N)<\sum_{j \in N} b_{j}^{v}$. Lemma 3.17 implies that $v$ is the sum of two quasi-balanced games $\alpha v^{S^{v}}$ and $v^{1}=v-\alpha v^{S^{v}}$ with $\alpha>0$ fulfilling (3.13) and (3.14). If $\alpha$ is chosen as large as possible, say $\alpha=\alpha^{1}$, then either (3.13) holds with equality or (3.14) holds with equality for at least one player in $S^{v}$. Let us consider these two cases into more detail. 
- $v(N)=\sum_{j \in N} b_{j}^{y}-\alpha^{1}\left|S^{v}\right|:$ we obtain $v^{1}(N)=\sum_{j \in N} b_{j}^{v^{1}}$, implying that $b^{v^{1}}$ is a core allocation of $v^{1}$. Hence, $v$ is the sum of $\alpha^{1} v_{S}$ and a game with a non-empty core, which can be further decomposed using (2.3).

- $a_{i}^{v}=b_{i}^{v}-\alpha^{1}$ for an $i \in S^{v}$ : thus there is a coalition $S$ containing player $i$ such that

$$
a_{i}^{v}-b_{i}^{v}=\max _{T \ni i}\left(v(T)-\sum_{j \in T} b_{j}^{v}\right)=v(S)-\sum_{j \in S} b_{j}^{v}=-\alpha^{1} .
$$

Now $|S| \leq|N|-2$ since otherwise

$$
-\alpha^{1}=v(S)-\sum_{j \in S} b_{j}^{v}=v(N)-\sum_{j \in S} b_{j}^{v}=-\alpha^{1}\left|S^{v}\right|,
$$

using (2.2); a contradiction. Therefore,

$$
\begin{aligned}
v^{1}(S)-\sum_{j \in S} b_{j}^{v^{1}} & =v(S)-\alpha^{1}\left(\left|S \cap S^{v}\right|-1\right)-\left(\sum_{j \in S} b_{j}^{v}-\alpha^{1}\left|S \cap S^{v}\right|\right) \\
& =v(S)-\sum_{j \in S} b_{j}^{v}+\alpha^{1} \\
& =0,
\end{aligned}
$$

implying $a_{i}^{v^{1}} \geq b_{i}^{v^{1}}$. The quasi-balancedness of $v^{1}$ now implies $a_{i}^{v^{1}}=$ $b_{i}^{v^{1}}$. For $j \notin S^{v}$ the equality $a_{j}^{v^{1}}=b_{j}^{v^{1}}$ easily follows from $a_{j}^{v}=b_{j}^{v}$. We, therefore, conclude that $S^{v^{1}}$ is a proper subset of $S^{v}$, and we continue with the decomposition

$$
v^{1}=v-\alpha^{1} v_{S^{v}}, v^{2}=v^{1}-\alpha^{2} v_{S^{1}}, \text { etc. . }
$$

until eventually $\left|S^{u^{k}}\right|<2$ or $v^{k}(N)=\sum_{j \in N} b_{j}^{v^{k}}$. Both cases have been discussed in lemma 3.16 .

This leads to the following procedure, with $v$ an arbitrary quasi-balanced game.

\section{Procedure 3.22}

Step 1 Let $k=0$ and $v^{0}$ the 0 -normalization of $v$. 
Step 2 Let $S^{k}=\left\{i \in N: a_{i}^{v^{k}}<b_{i}^{v^{k}}\right\}$.

If $\left|S^{k}\right|<2$ or $\sum_{j \in N} v_{j}^{k}=\sum_{j \in N} b_{j}^{v^{k}}$

then $v^{k}$ is decomposed according to (2.3); STOP.

Step 3 Let $\alpha^{k+1}$ denote the minimum of $\left(\sum_{j \in N} b_{j}^{k^{k}}-v^{k}(N)\right) /\left|S^{k}\right|$ and the differences $b_{i}^{v^{k}}-a_{i}^{v^{k}}, i \in S^{k}$.

Let $v^{k+1}=v^{k}-\alpha^{k+1} v^{s^{k}}$.

Step 4 Increase $k$ by 1 , and GOTO step 2.

Theorem 3.23 Procedure 3.22 terminates after a finite number of loops. It represents a quasi-balanced game as a non-negative weighted sum of the generating games of $\mathrm{Q}^{N}$ mentioned in theorem 3.19 .

Proof: In the introduction to procedure 3.22 it has been shown that the sets of players $S^{k}$ are shrinking: $N \supseteq S^{0} \supset S^{1} \supset \ldots$ Therefore, step 2 is passed through only finitely many times which proves that the procedure eventually terminates.

It is obvious that the required representation arises from the procedure, together with the usage of the decomposition in (2.3) (in which either $a^{v}$ or $b^{v}$ can be chosen as the core allocation $x$ ) and the equality $v=v_{0}+u=v_{0}+\sum_{j \in N} v(\{j\}) u^{\{j\}}$, with $u$ the additive game associated to the allocation $(v(\{i\}))_{i \in N}$.

\subsection{A comparison of considered classes of games}

In the previous sections we characterized several classes of games by presenting a generating set or by decomposing it into (an algebraic sum of) subclasses for which generating sets were available. Here we will summarize most of these results. Furthermore, the inclusion relations are discussed into more detail.

\subsubsection{A review}

In the table below we gather some of the results of the previous sections. The considered games are defined as follows, with player set $N$ consisting of at least four players: 
$\mathbb{1}^{S}, \emptyset \neq S \subseteq N$, are the unity games, assigning worth 1 to the coalition

$S$ and 0 to the other coalitions;

${ }_{u^{\{i\}}}=\sum_{S \ni i} \mathbb{1}^{S}, i \in N$, are the dictator games;

$\hat{w}$ is the simple game $\sum_{S:|S| \geq|N|-1} \mathbb{1}^{S}$;

$w^{S}, S \subseteq N,|S| \geq 2$, assigns to non-empty coalitions $T$. the worth

$|S \cap T|-1$ whenever $S \cap T \neq \emptyset$, and 0 otherwise;

$v^{S}, \emptyset \neq S \subseteq N$, equals $-\mathbf{1}^{N \backslash\{i\}}$ if $S=\{i\}, i \in N$, and for the other

coalitions $S \neq \emptyset$ it is defined to be equal to $w^{S}-(|S|-1) \hat{w}$.

In order to elucidate the relationships consider the following cones of games. These sets form basic components with which the considered classes of games can be composed.

$$
\begin{aligned}
& \mathbf{A}=\operatorname{Cone}\left(\left\{u^{\{i\}}: i \in N\right\} \cup\left\{-u^{\{i\}}: i \in N\right\}\right)=\mathbf{A}^{N} \text {, } \\
& \mathrm{B}=\mathrm{Cone}\left(\left\{-\mathbb{1}^{S}: S \subset N, 2 \leq|S| \leq|N|-2\right\}\right) \text {, } \\
& \mathbf{C}=\operatorname{Cone}\left(\left\{-\mathbb{1}^{\{i\}}: i \in N\right\}\right) \text {, } \\
& \mathbf{D}=\operatorname{Cone}\left(\left\{-\mathbb{1}^{N \backslash\{i\}}: i \in N\right\}\right) \text {, } \\
& \mathbf{E}=\operatorname{Cone}\left(\left\{w^{S}: S \subseteq N, 2 \leq|S|\right\}\right)=\mathbf{A C}^{N} \text {, and } \\
& \mathbf{F}=\operatorname{Cone}\left(\left\{v^{s}: S \subseteq N, 2 \leq|S|\right\}\right) \text {. }
\end{aligned}
$$

The following correspondences hold.

\begin{tabular}{|l|c|l|l|}
\hline game property & class & characterization & theorem \\
\hline & & & \\
additive & $\mathbf{A}^{N}$ & $\mathbf{A}$ & 1.16 \\
& $\mathbf{D}^{N}$ & $\mathbf{A}+\mathbf{B}+\mathbf{C}$ & 2.2 \\
& $\mathbf{H}^{N}$ & $\mathbf{A}+\mathbf{B}+\mathbf{D}$ & 2.3 \\
& $\mathbf{C C}^{N}$ & $\mathbf{F}$ & 2.16 \\
maximally blocked & $\mathbf{M B}^{N}$ & $\mathbf{A}+\mathbf{B}+\mathbf{C}+\mathbf{S p a n}\left(\left\{w^{N}\right\}\right)$ & 3.6 \\
1-convex & $\mathbf{C}^{N}$ & $\mathbf{A}+\mathbf{B}+\mathbf{C}+\mathbf{C o n e}\left(\left\{w^{N}\right\}\right)$ & 3.6 \\
non-empty core & $\mathbf{C}^{N}$ & $\mathbf{A}+\mathbf{B}+\mathbf{C}+\mathbf{D}$ & 2.1 \\
quasi-balanced & $\mathbf{Q}^{N}$ & $\mathbf{A}+\mathbf{B}+\mathbf{C}+\mathbf{D}+\mathbf{F}$ & 3.19 \\
semiconvex & $\mathbf{S C}^{N}$ & $\mathbf{A}+\mathbf{B}+\mathbf{D}+\mathbf{E}+\mathbf{C o n e}(\{-\hat{w}\})$ & 3.12 \\
semibalanced & $\mathbf{S}^{N}$ & $\mathbf{A}+\mathbf{B}+\mathbf{C}+\mathbf{C o n e}(\{-\hat{w}\})$ & 3.1 \\
\hline
\end{tabular}

The clan games and the restrictions of the above classes to the 0-normalized and the non-negative games are treated further on. 
Notice that the intersection of each two distinct components, used in the table, only consists of the zero game, except the intersection of each two of the sets $\mathbf{E}$, Cone $\left(\left\{w^{N}\right\}\right)$ and $\operatorname{Span}\left(\left\{w^{N}\right\}\right)$.

The following inclusion relations now follow immediately:

$$
\begin{aligned}
& \mathrm{AC}^{N} \subset \mathrm{SC}^{N} \\
& \cap \\
& \mathbf{A}^{N} \subset \mathbf{D}^{N} \subset \mathbf{1}^{N} \subset \mathbf{C}^{N} \subset \mathbf{Q}^{N} \subset \mathbf{S}^{N} \\
& \stackrel{\cap}{\mathbf{H}^{N}} \quad \mathbf{M B}^{N}
\end{aligned}
$$

The 0-normalized case

All components used in the above table are contained in the class of D-normalized games, except for $\boldsymbol{A}$. Letting

$$
\mathbf{A}_{0}=\operatorname{Cone}\left(\left\{u^{\{i\}}-\mathbf{1}^{\{i\}}: i \in N\right\}\right),
$$

the 0-normalized case practically equals the general case, with $\mathbf{A}_{0}$ instead of $A$, and with the deletion of $\mathbf{C}$ :

\begin{tabular}{|c|l|l|}
\hline class & characterization & theorem/cor. \\
\hline & & \\
$\mathbf{D}_{0}^{N}$ & $\mathbf{A}_{0}+\mathbf{B}$ & 2.5 \\
$\mathbf{H}_{0}^{N}$ & $\mathbf{B}+\mathbf{D}$ & 2.5 \\
$\mathbf{A C}_{0}^{N}=\mathbf{A C}^{N}$ & $\mathbf{F}$ & 2.16 \\
$\mathbf{M B}_{0}^{N}$ & $\mathbf{A}_{0}+\mathbf{B}+\mathbf{S p a n}\left(\left\{w^{N}\right\}\right)$ & 3.8 \\
$\mathbf{I C}_{0}^{N}$ & $\mathbf{A}_{0}+\mathbf{B}+\mathbf{C o n e}\left(\left\{w^{N}\right\}\right)$ & 3.8 \\
$\mathbf{C}_{0}^{N}$ & $\mathbf{A}_{0}+\mathbf{B}+\mathbf{D}$ & 2.5 \\
$\mathbf{Q}_{0}^{N}$ & $\mathbf{A}_{0}+\mathbf{B}+\mathbf{D}+\mathbf{F}$ & 3.18 \\
$\mathbf{S C}_{0}^{N}$ & $\mathbf{B}+\mathbf{D}+\mathbf{E}+\mathbf{C o n e}(\{-\hat{w}\})$ & $\mathbf{3 . 1 4}$ \\
$\mathbf{S}_{0}^{N}$ & $\mathbf{A}_{0}+\mathbf{B}+\operatorname{Cone}(\{-\hat{w}\})$ & $\mathbf{3 . 3}$ \\
\hline
\end{tabular}

Let us consider intersections of some of the classes in the above table.

- $\mathbf{M B}_{0}^{N} \cap \mathbf{S C}_{0}^{N} \subset \mathbf{1 C}_{0}^{N}$ : Consider the game $v \in \mathbf{M B}_{0}^{N} \cap \mathbf{S C}_{0}^{N}$. From the maximal blockedness and the semiconvexity property we gain $0=$ $v(\{i\}) \leq v(N)-\sum_{j \in N \backslash\{i\}} b_{j}^{v}$ and $0=v(\{i\})=a_{i}^{v} \geq v(N)-\sum_{j \in N \backslash\{i\}} b_{j}^{v}$ respectively, and, therefore, $v(N)=\sum_{j \in N \backslash(i\}} b_{j}^{v}$ for each player $i$. The semiconvexity of $v$ implies $0=v(\{i\}) \leq b_{i}^{v}$ (since $v$ is semibalanced) 
and $v(S) \leq v(\{i\})+\sum_{j \in N \backslash\{i\}} b_{j}^{v}=\sum_{j \in N \backslash\{i\}} b_{j}^{v}$ for each coalition $S$ and player $i \in S$. It follows that $b^{v}-b_{i}^{t} \chi^{\{i\}}$ is a core allocation of $v$ implying

$$
\operatorname{MCore}(v)=\operatorname{Conv}\left(\left\{b^{v}-b_{i}^{v} \chi^{\{i\}}: i \in N\right\} \subseteq \operatorname{Core}(v) .\right.
$$

In view of theorem 1.6 we conclude that $v$ is 1 -convex, i.e., $\mathrm{MB}_{0}^{N} \mathrm{n}$ $\mathbf{S C}_{0}^{N} \subset \mathbf{1 C}_{0}^{N}$.

- $\mathrm{MB}_{0}^{N} \cap \mathbf{S}_{0}^{N}=\mathbf{1 C}_{0}^{N}$ : obviously, $\mathbf{1 C}_{0}^{N} \subseteq \mathbf{M B}_{0}^{N} \cap \mathrm{S}_{0}^{N}$ holds. For a game $w \in \mathbf{M B}_{0}^{N} \cap \mathbf{S}_{0}^{N}$ the marginal gap $\gamma^{v}=\sum_{j \in N} b_{j}^{v}-v(N)$ is non-negative and together with the maximal blockedness of $v$ this implies that $v$ is 1-convex.

- The intersection of $\mathbf{S C}_{0}^{N}$ and $\mathbf{C}_{0}^{N}$ is not equal to $\mathbf{C}_{0}^{N}$ and it obviously enclose the set $\mathbf{B}+\mathbf{D}+\mathbf{E}=\mathbf{H}^{N}+\mathbf{A C}^{N}$. It includes, however, more games as can be shown as follows. Consider the 0-normalized semiconvex game $w^{N}-\alpha \hat{w}$, with $\alpha \in I R_{+}$. It is not an element of $\mathbf{H}^{N}+\mathbf{A C}^{N}$. The allocation $|N|-1-\alpha /|N| \chi^{N}$ is efficient for this game. Furthermore, it is a core allocation whenever $\alpha$ is suitably small as can easily be checked.

- The two sets $\mathbf{H}^{N}$ and $\mathbf{A C ^ { N }}$ are also included in $\mathbf{Q}_{0}^{N}$. The intersection of $\mathbf{S C}^{N}$ and $\mathbf{Q}^{N}$, however, unequals the sum $\mathbf{H}^{N}+\mathbf{A C}^{N}$ as is illustrated by the four-person game $v$ given by $v(S)=0,1,-1,0$ for $|S|=1,2,3,4$ respectively. We obtain that $b^{v}=\chi^{N}, a^{v}=0$ and thus, the game $v$ is both semiconvex and quasi-balanced. Nevertheless, the game $v$ cannot be an element of the sum $\mathbf{H}^{N}+\mathbf{A C}^{N}$ because its core is empty.

The restriction to the non-negative games

The following table summarizes the results with respect to the classes of non-negative games which have been considered in the previous sections. We recall that

$u^{S}, \emptyset \neq S \subseteq N$, are the unanimity games, assigning 1 to coalitions $T$ containing $S$ and 0 otherwise;

$\operatorname{car}^{S}, \emptyset S \subseteq N$, is the cardinality game defined by $\operatorname{car}^{S}=|T \cap S|$ for each coalition $T$;

vone denotes the all winning game assigning 1 to each non-empty coalition; 
$\operatorname{mix}_{\mathbf{\Omega}}(v), v \in \mathbf{G}^{N}$ and $\Omega \subseteq 2^{N}$, denotes the game which equals $v$ on the coalitions in $\Omega$ and 0 otherwise.

The table has to be interpreted as follows. In each row the class in the first column equals the cone generated by the games $\operatorname{mix}_{n}(v)$, with $v$ a game from the column denoted by $W$, and $\Omega$ a set of coalitions containing the coalitions which do not obey the restrictions in the column 'possibly 0-valued'.

\begin{tabular}{|c|c|c|c|}
\hline class & $W$ & possibly 0-valued & theorem \\
\hline $\begin{array}{c}\mathbf{C}_{+}^{N} \\
\mathbf{D}_{+}^{N} \\
\mathbf{H}_{+}^{N} \\
\mathbf{1 C}_{+}^{N} \\
\mathbf{M B}_{+}^{N} \\
\mathbf{C L A N}^{N, C} \\
\mathbf{S}_{+}^{N}\end{array}$ & $\begin{array}{c}u^{\{i\}}, i \in N \\
u^{\{i\}}, i \in N \\
u^{\{i\}}, i \in N \\
u^{\{i\}}, i \in N, w^{N} \\
u^{\{i\}}, v_{\text {one }}-u^{\{i\}}, i \in N, w^{N} \\
u^{C \cup\{i\}}, i \in N \backslash C, u^{C} \\
\operatorname{car}^{S}-(|S|-1) \hat{w}, S \neq \emptyset\end{array}$ & $\begin{array}{l}\text { the coalitions } T \neq N \\
\qquad \begin{array}{c}|T|<|N|-1 \\
T \neq N \text { and }|T|>1 \\
|T|<|N|-1 \\
|T|<|N|-1 \\
|T|<|N|-1 \\
|T|<|N|-1\end{array}\end{array}$ & $\begin{array}{l}2.8 \\
- \\
- \\
3.10 \\
3.9 \\
3.11 \\
3.5 \\
\end{array}$ \\
\hline
\end{tabular}

\subsubsection{Spanning sets for the game space}

Some of the above mentioned games form a spanning set for the whole class $\mathbf{G}^{N}$ of games. Well-known is that the set of unanimity games has this property. It even forms a base (see for example Shapley [1953] and Dubey [1975]). This is also the case for the games $w^{s}, S \subseteq N$, and the games $v^{S}$, $S \subseteq N$ (where $w^{\{i\}}$ and $v^{\{i\}}, i \in N$, are defined appropiately).

In order to prove these results we consider square matrices of size $2^{|N|-1}$; it is assumed that each of the rows or columns of the considered matrices correspond to one of the non-empty coalitions.

The following matrices are considered: $A=\left(a_{S T}\right), B=\left(b_{S T}\right), C=\left(c_{S T}\right)$ and $D=\left(d_{S T}\right)$ defined by ( $S$ and $T$ are assumed to be non-empty)

$$
\begin{aligned}
& a_{S T}=\left\{\begin{array}{rl}
1 & \text { if } S \subseteq T, \\
0 & \text { otherwise; }
\end{array} b_{S T}=\left\{\begin{aligned}
1 & \text { if } S \subseteq T,|T \backslash S| \text { is even, } \\
-1 & \text { if } S \subseteq T,|T \backslash S| \text { is odd, } \\
0 & \text { otherwise; }
\end{aligned}\right.\right.
\end{aligned}
$$




$$
\begin{aligned}
& c_{S T}=\left\{\begin{aligned}
1 & \text { if } S \cap T \neq 0 \\
0 & \text { otherwise; }
\end{aligned}\right. \\
& d_{S T}=\left\{\begin{aligned}
(-1)^{|N|+1} & \text { if } S \cup T=N,|T \backslash S| \text { is even, } \\
-(-1)^{|N|+1} & \text { if } S \cup T=N,|T \backslash S| \text { is odd } \\
0 & \text { otherwise. }
\end{aligned}\right.
\end{aligned}
$$

Observe that the rows of matrix $A$ correspond to the unanimity games and the rows of $C$ are the airport cost functions $c_{\chi} s$, which is denoted here by $c_{S}, \emptyset \neq S \subseteq N$. (see section 1.2.6).

The matrices $C$ and $D$ are used implicitly and in an adjusted way in Muto, Potters and Tijs [1989] (see also the definition of derivatives in Shapley [1971]).

We will prove that the matrices $A$ and $B$ and the matrices $C$ and $D$ are each other's inverse. The following well-known result will be very useful.

Lemma $3.24 \sum_{S: S \subseteq T}(-1)^{|S|}=0$ for all $T \subseteq N, T \neq \emptyset$.

Proof: $\sum_{S: S \subseteq T}(-1)^{|S|}=\sum_{k=0}^{|T|}(-1)^{k}\left(\begin{array}{c}|T| \\ k\end{array}\right)=(1+(-1))^{|T|}=0$.

Lemma 3.25 $A B=I$ with $I$ denoting the unity matrix.

Proof: Notice that $b_{S T}=(-1)^{|S|-|T|_{a}}$ for all non-empty coalitions $S$ and $T$. Then

$$
\begin{aligned}
(A B)_{S T} & =\sum_{U \subseteq N}(-1)^{|U|-|T|} a_{S U} a_{U T} \\
& =\sum_{U: S \subseteq U \subseteq T}(-1)^{|U|-|T|} \\
& =\left\{\begin{array}{cl}
1 & \text { if } S=T, \\
0 & \text { if } S \nsubseteq T, \\
(-1)^{|S|-|T|} \sum_{X \subseteq T \backslash S}(-1)^{X} & \text { if } S \subset T .
\end{array}\right.
\end{aligned}
$$

Using lemma 3.24 we obtain $A B=I$.

Lemma 3.26 $D C=I$.

Proof: Let $S$ and $T$ be two non-empty coalitions. Notice that $d_{S T}=$ $(-1)^{|N|+1+|S|-|T|}$ for coalitions $S, T$ with $S \cup T=N$. Then

$$
(D C)_{S T}=\sum_{U \subseteq N} d_{S U} c_{U T}=\sum_{U: S \cup U=N, U \cap T \neq \emptyset}(-1)^{|N|+1+|S|-|U|} .
$$


If $T \& S$ then $U \cap T \neq \emptyset$ for all $U \subseteq N$ with $S \cup U=N$. Thus,

$$
\begin{aligned}
(D C)_{S T} & =\sum_{U: S \cup U=N}(-1)^{|N|+1+|S|-|U|} \\
& =\sum_{X \subseteq S}(-1)^{|N|+1+|S|-|X \cup N| S \mid} \\
& =\sum_{X \subseteq S}(-1)^{|N|+1+|S|-|X|-(|N|+|S|)} \\
& =\sum_{X \subseteq S}(-1)^{1-|X|} \\
& =-\sum_{X \subseteq S}(-1)^{|X|},
\end{aligned}
$$

which equals 0 according to lemma 3.24 .

For $T \subset S$ the set $\{U \subseteq N: S \cup U=N, U \cap T \neq \emptyset\}$ equals $\{X \cup Y \cup N \backslash S$ : $X \subseteq T, X \neq \emptyset, Y \subseteq S \backslash T\}$ and, thus,

$$
\begin{aligned}
(D C)_{S T} & =\sum_{X \subseteq T, X \neq \emptyset} \sum_{Y \subseteq S \backslash T}(-1)^{|N|+1+|S|-|X \cup Y \cup N \backslash S|} \\
& =\sum_{X \subseteq T, X \neq \emptyset} \sum_{Y \subseteq S \backslash T}(-1)^{|N|+1+|S|-|X|-|Y|-|N|+|S|} \\
& =\sum_{X \subseteq T, X \neq \emptyset}(-1)^{1-|X|} \sum_{Y \subseteq S \backslash T}(-1)^{2|S|-|Y|} \\
& =\sum_{X \subseteq T, X \neq \emptyset}(-1)^{1-|X|} \sum_{Y \subseteq S \backslash T}(-1)^{|Y|}
\end{aligned}
$$

which once again equals 0 according to lemma 3.24 .

It remains to consider the case $S=T$.

The set $\{U: S \cup U=N, U \cap T \neq \emptyset\}$ now equals $\{X \cup N \backslash S: X \subseteq S, X \neq \emptyset\}$ and, thus,

$$
\begin{aligned}
(D C)_{s S} & =\sum_{X \subseteq S, X \neq \emptyset}(-1)^{|N|+1+|S|-|X \cup N \backslash S|} \\
& =-(-1)^{|N|+1+|S|-|N|+|S|}+\sum_{X \subseteq S}(-1)^{|N|+1+|S|-|X|-|N|+|S|} \\
& =1+\sum_{X \subseteq S}(-1)^{1-|X|+2|S|} \\
& =1-\sum_{X \subseteq S}(-1)^{|X|}
\end{aligned}
$$


Using lemma 3.24 this equals 1 . We conclude that $D C=I$.

Clearly, the set $\left\{x A: x \in I R_{+}^{2^{N}} \backslash\{\theta\}\right\}$ corresponds to the cone generated by the unanimity games. For a game $v$ the assertion $v^{t} B \geq 0^{t}$ is therefore equivalent with $v \in \operatorname{Cone}\left(\left\{u^{S}: \emptyset \neq S \subseteq N\right\}\right)$. The entry corresponding to coalition $U$ in $v^{t} B$ equals the so-called $U$-derivative

$$
\Delta_{U}^{u}(S)=\sum_{T: S \subseteq U}(-1)^{|U|-|T|} v(S \cup T) \text { with } S \subseteq N \backslash U .
$$

See also Shapley [1953]. This quantity corresponds also to the so-called divident of the coalition $S$ (Harsanyi [1963]). Thus,

Corollary 3.27 A game $v$ is a non-negative weighted sum of the unanimity games if and only if all $U$-derivatives of $v$ are non-negative.

Our prime intention here is to consider the dimensionality of several of the generating sets, which are presented.

Theorem 3.28 The sets $\left\{u^{S}: \emptyset \neq S \subseteq N\right\}$ and $\left\{c_{S}: \emptyset \neq S \subseteq N\right\}$ are bases of $\mathbf{G}^{N}$.

Proof: The set $\left\{u^{S}: \emptyset \neq S \subseteq N\right\}$ consists of the rows of matrix $A$ which forms a maximal independent set of elements in $I R^{2^{N}} \backslash\{\emptyset\}$ according to lemma 3.25. This proves that $\left\{u^{S}: \emptyset \neq S \subseteq N\right\}$ is a base of $\mathbf{G}^{N}=I R^{2^{N}} \backslash\{\theta\}$.

In a similar way, using lemma 3.26 , one proves that $\left\{c_{S}: \emptyset \neq S \subseteq N\right\}$ is a base of $\mathrm{G}^{N}=I R^{2^{N} \backslash\{\phi\}}$.

The airport cost savings games $w^{S}, S \subseteq N,|S|>1$, are defined by $w^{S}=$ $\sum_{j \in S} u^{\{j\}}-c_{S}$. Let us consider here $w^{\{i\}}$ to be defined as the semiconvex game $-\mathbf{1}^{N \backslash\{i\}}, i \in N$. Notice that the definition of the quasi-balanced games $\left.v^{S}, S \subseteq N\right\},|S|>1$, as the games $w^{S}-(|S|-1) \hat{w}$ naturally extends to $v^{\{i\}}=-\mathbb{1}^{N \backslash\{i\}}, i \in N$, which fully agrees with our definition of $v^{\{i\}}$ as is done in section 3.5.2.

Suppose that the games $w^{S}, \neq \subseteq \subseteq N$, are dependent, i.e., consider the linear combination $\sum_{\emptyset \neq S \subseteq N} \alpha_{S} w^{S}$ to be the all losing game. We will prove that the scalars $\alpha_{S}, \emptyset \neq S \subseteq N$, are 0 . Notice that the marginal contribution allocation of the all losing game is zero and $b^{w^{s}}=\chi^{S}, \neq S \subseteq N$; this implies

$$
0=\sum_{\emptyset \neq S \subseteq N} \alpha_{S} \chi^{S}=\left(\sum_{S \ni i} \alpha_{S}\right)_{i \in N}
$$


Then

$$
\begin{aligned}
& 0=\sum_{\emptyset \neq S \subseteq N} \alpha_{S} w^{S} \\
& =\sum_{\emptyset \neq S \subseteq N} \sum_{j \in S} \alpha_{S} u^{\{j\}}-\sum_{\emptyset \neq S \subseteq N} \alpha_{S} c_{S}
\end{aligned}
$$

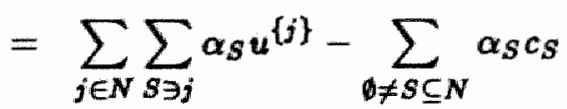

$$
\begin{aligned}
& =-\sum_{\emptyset \neq S \subseteq N} \alpha_{S} c_{S} \text {. }
\end{aligned}
$$

Since $\left\{c_{S}: \emptyset \neq S \subseteq N\right\}$ is a base we must have $\alpha_{S}=0$ for all $\emptyset \neq S \subseteq N$. Therefore, $\left\{w^{S}: \emptyset \neq S \subseteq N\right\}$ is an independent set of $2^{|N|}-1$ games in the game space.

Similarly, one proves that this holds also for the set of games $v^{S}, \emptyset \neq$ $S \subseteq N\}$.

Corollary 3.29 The sets $\left\{w^{S}: \emptyset \neq S \subseteq N\right\}$ and $\left\{v^{S}: \emptyset \neq S \subseteq N\right\}$ are bases of $\mathbf{G}^{N}$.

\section{Bases of semibalanced games and of 1-convex games}

Consider the $2^{|N|}-1$ semibalanced games $u^{\{i\}}, i \in N,-\mathbb{1}^{\{i\}}, S \subset N$, $|S|<|N|-1$, and $-\hat{w}$, which are contained in a generating set of $\mathbf{S}^{N}$, presented in theorem 3.1. These games also form a base of $\mathrm{G}^{N}$. This follows easily from the observations

$$
-\mathbb{1}^{N \backslash\{i\}}=u^{\{i\}}+\sum_{S \ni i,|S|<|N|-1}\left(-\mathbb{1}^{S}\right)-\hat{w}, \quad i \in N,
$$

and

$$
\mathbf{1}^{N}=\sum_{j \in N} u^{[j\}}+\sum_{s \subset N_{,}|S|<|N|-1}|S|\left(-\mathbb{1}^{S}\right)-(|N|-1) \hat{w} .
$$

Furthermore, consider the above set $W$ of games with the airport cost savings game $w^{N}$ instead of $-\hat{w}$. In theorem 3.7 these games form a generating set of $1 \mathrm{C}^{N}$, together with the games $-u^{\{i\}}, i \in N$. The equality

$$
\begin{aligned}
w^{N} & =\operatorname{car}^{N}-v_{\text {one }} \\
& =\sum_{j \in N} u^{\{i\}}+\sum_{S:|S|<|N|-1}\left(-\chi^{S}\right)(-\hat{w})
\end{aligned}
$$


implies that $-\hat{w}$ can be written as a combination of the games in $W$. Therefore, $W$ is a base of the game space.

The following table lists the bases we considered here.

\begin{tabular}{|l|l|}
\hline base & game property \\
\hline & \\
$u^{\{i\}}, i \in N,-\mathbb{1}^{S}, 1 \leq|S|<|N|-1,-\hat{w}$ & semibalanced \\
$u^{\{i\}}, i \in N,-\mathbb{1}^{S}, 1 \leq|S|<|N|-1, w^{N}$ & 1-convex \\
$u^{S}, \emptyset \neq S \subseteq N$, & convex \\
$c_{S}, \emptyset \neq S \subseteq N$, & airport cost f. \\
$w^{S}, \emptyset \neq S \subseteq N$, & semiconvex \\
$v^{S}, \emptyset \neq S \subseteq N$, & quasi-balanced \\
\hline
\end{tabular}

\subsubsection{The cost games of the considered games}

Using the gap function some of the introduced types of savings games have a more simple expression as a cost game (see also Driessen [1988]). The semibalancedness of a game $v$, for example, corresponds exactly to the nonnegativity of the corresponding cost game $g^{v}$ and the maximal blockedness of $v$ is equivalent to

$$
g^{v}(N) \leq g^{v}(S) \text { for each non-empty coalition } S,
$$

i.e., the costs for the grand coalition are the lowest among the costs of all non-empty coalitions. For a 1-convex game $v$ the corresponding cost game $g^{v}$ has lowest non-negative costs for the grand coalition and for a semiconvex game the lowest non-negative costs are attained by the one-person coalitions, i.e.,

$$
0 \leq g^{\nu}(\{i\}) \leq g^{\nu}(S) \text { for all } i \in N \text { and } S \subseteq N \text { with } i \in S .
$$

As we saw in chapter 1 the quasi-balancedness of a game $v$ is equivalent to $v \in \mathbf{S}^{N}$ and $\sum_{j \in N} a_{j}^{v} \leq v(N)$. Using the definition of the maximal remainder allocation the latter inequality is equivalent to

$$
\sum_{j \in N} b_{j}^{v}-v(N) \leq \sum_{j \in N} \min _{S \ni j}\left(\sum_{i \in S} b_{i}^{v}-v(S)\right)
$$

i.e.,

$$
g^{v}(N) \leq \sum_{j \in N} \min _{S \ni j} g^{v}(S)
$$


Let $e^{w} \in I R^{N}$ of a game $w$ denote the concession allocation defined by

$$
e_{i}^{\psi}=\min _{S \ni i} w(S) \quad \text { for } i \in N \text {; }
$$

the amount $e_{i}^{w}$ of a cost game $w$ represents the minimal amount of costs that player $i$ will be charged, possibly together with other players. With this notation we conclude that a game $v$ is quasi-balanced if and only if its gap function is non-negative and

$$
g^{v}(N) \leq \sum_{j \in N} e_{j}^{g^{*}}
$$

One simply shows that for a game $v$ the difference between $b^{v}$ and $a^{v}$ equals the concession allocation of the corresponding cost game $g^{*}$ (cf. Driessen and Tijs [1983]).

The following table shows the correspondences between types of savings games and cost games.

\begin{tabular}{|l|l|}
\hline $\begin{array}{l}\text { savings games } \\
\text { (0-normalized) }\end{array}$ & $\begin{array}{l}\text { cost games } \\
\text { (zero marginal contribution allocation) }\end{array}$ \\
\hline $\begin{array}{l}\text { semibalancedness } \\
\text { maximal blockedness } \\
\text { 1-convexity } \\
\text { semiconvexity } \\
\text { quasi-balancedness }\end{array}$ & $\begin{array}{l}\text { non-negativity } \\
\text { lowest costs for } N \\
\text { lowest non-negative costs for } N \\
\text { lowest non-negative costs for }\{i\}, i \in N \\
\text { non-negativity, costs for } N \text { do not exceed the } \\
\text { sum of the minimal consessions of the players. }\end{array}$ \\
\hline
\end{tabular}

The gap function, as discussed in section 1.2.6, is a 1-1 mapping between 0 -normalized games and cost games $c$ with $b^{c}=0$. The corresponding cost games associated to the savings games in the generating set of an arbitrary cone $\mathbf{W}_{0}^{N}$ of 0-normalized games therefore forms a generating set for $g\left(\mathbf{W}_{0}^{N}\right)$. The extremality of the games in $W_{0}^{N}$ moreover induces the extremality of the corresponding cost games in $g\left(\mathbf{W}_{0}^{N}\right)$. The table below contains the savings games and corresponding cost games of the generating sets of the classes of 0-normalized games, which are considered in this chapter. It is also indicated in which class it is contained ('e' for extreme, ' $y$ ' for contained, and ' $n$ ' for not contained) The player set $N$ is assumed to consist of at least 4 players. In the table $S$ denotes a coalition of at least 2 players. 


\begin{tabular}{|c|c|c|c|c|c|c|}
\hline savings game & $\mathbf{S}_{0}^{N}$ & $\mathrm{MB}_{0}^{N}$ & $1 \mathrm{C}_{0}^{N}$ & $\mathrm{SC}_{0}^{\mathrm{N}}$ & $Q_{0}^{N}$ & cost game \\
\hline $\begin{array}{c}w^{\{i\}}-\mathbb{1}\{i\} \\
-\mathbb{1}^{N \mid\{i\}} \\
-\mathbb{H}^{S}(|S|<|N|-\mathbb{L}) \\
-\hat{w}^{N} \\
w^{N} \\
-w^{N} \\
w^{S}(|S|<|N|) \\
v^{S}\end{array}$ & $\begin{array}{l}\mathbf{e} \\
\mathbf{n} \\
\mathbf{e} \\
\mathbf{e} \\
\mathbf{y} \\
\mathbf{n} \\
\mathbf{y} \\
\mathbf{y}\end{array}$ & $\begin{array}{l}y \\
y \\
y \\
y \\
y \\
y \\
y \\
n\end{array}$ & $\begin{array}{l}\text { e } \\
\text { e } \\
\text { n } \\
\text { n } \\
\text { y } \\
\text { n } \\
\text { y } \\
\text { n }\end{array}$ & $\begin{array}{l}\mathbf{n} \\
\mathbf{n} \\
\mathbf{e} \\
\mathbf{e} \\
\mathbf{e} \\
\mathbf{n} \\
\mathbf{e} \\
\mathbf{n}\end{array}$ & $\begin{array}{l}\mathrm{e} \\
\mathrm{e} \\
\mathrm{e} \\
\mathrm{n} \\
\mathbf{y} \\
\mathrm{n} \\
\mathrm{e} \\
\mathrm{e}\end{array}$ & $\begin{array}{c}\mathbf{1}^{(i)} \\
+\mathbf{1}^{(i)} \\
\mathbf{1}^{s} \\
\hat{w} \\
v_{\text {one }} \\
-v_{\text {one }} \\
c_{\chi^{s}} \\
c_{x}+(|S|-1) \hat{w}\end{array}$ \\
\hline
\end{tabular}

We conclude that

- $\left\{c \in \mathbf{G}^{N}: b^{c}=0, c(S) \geq 0\right.$ for all $\left.S \subseteq N\right\}$ is generated by the extreme directions $\mathbf{1}^{\{i\}}, i \in N, \mathbb{1}^{S}, S \subset N, 1<|S|<|N|-1$, and $\hat{w}$;

- $\left\{c \in \mathbf{G}^{N}: b^{c}=0, c(S) \geq c(N)\right.$ for all $\left.S \subseteq N\right\}$ is generated by the games $\mathbb{1}^{\{i\}}, i \in N, \mathbb{1}^{s}, S \subset N, 1<|S|<|N|-1$, vone, and-vone;

- $\left\{c \in \mathbf{G}^{N}: b^{c}=0, c(S) \geq c(N) \geq 0\right.$ for all $\left.S \subseteq N\right\}$ is generated by the extreme directions $\mathbb{1}^{\{i\}}, i \in N, \mathbb{1}^{S}, S \subset N, 1<|S|<|N|-1$, and vone;

- $\left\{c \in \mathbf{G}^{N}: b^{c}=0, c(S) \geq c(\{i\})\right.$ for $i \in N$ and $S \subseteq N$ with $\left.i \in S\right\}$ is generated by the extreme directions $u^{\{i\}}+\mathbf{1}^{N \backslash\{i\}}, i \in N, \mathbf{1}^{s}, S \subset N$, $1<|S|<|N|-1, \hat{w}$, wone and $c_{\chi} s, S \subset N, 1<|S|$;

- $\left\{c \in \mathbf{G}^{N}: b^{c}=0, c(S) \geq 0\right.$ for all $S \subseteq N$ and $\left.c(N) \leq \sum_{j \in N} e_{j}^{c}\right\}$ is generated by the extreme directions $\mathbf{1}^{\{i\}}, u^{\{i\}}+\mathbb{1}^{N \backslash\{i\}}, i \in N, \mathbf{1}^{S}$, $S \subset N, 1<|S|<|N|-1$, and $c_{\chi}+(|S|-1) \hat{w}, S \subseteq N, 1<|S|$. 



\section{Chapter 4}

\section{Minimum closed cones of}

\section{games}

\subsection{Introduction}

In this chapter we will discuss cones of games with the property that for each two elements also the coalition-wise minimum is a member. Well-known examples are the class $\mathbf{S A}^{N}$ of superadditive games, the class $\mathbf{B}^{N}\left(\mathbf{T B}^{N}\right)$ of (totally) balanced games and the class $\mathbf{M N}^{N}$ of monotonic games. The minimum closedness property holds also for the restriction of these classes to the non-negative games.

The main result of this chapter concerns a set of conditions to guarantee the extremality of a game in a minimum closed cone. Extra conditions will be put on the cone itself such as the monotonicity of its members. One of the conditions in order to assert the extremality of a game is the extremality of some of its subgames.

The results presented here are inspired by a partial characterization of the extreme superadditive games in $\mathbf{S A}_{+}^{N}$, discussed in Derks [1987b]. The applied game theoretic notions such as essential coalitions, decomposability and minimum covers appeared to be based only on the minimum closedness nature of the class of non-negative superadditive games.

\section{The contents}

The contents of this chapter are as follows. We will start with a general discussion of cones (of games) with the minimum closedness property. Normal vectors of adjacent hyperplanes for minimum closed cones appear to have at most one positive entry. With respect to a minimum closed cone of 
games the notions of essentiality of a coalition, the minimum cover and the decomposability of a game into a sum of subgames are introduced and discussed in detail. Section 2 ends with a discussion of our main result. Section 3 is devoted to its application on the cone $\mathbf{S A}_{+}^{N}$. With respect to extreme games of $\mathbf{S A}_{+}^{N}$ some results may be found in literature. A survey of these results is added to section 3 .

\subsection{Minimum closed cones of games}

Let us first give some general results on minimum closed cones.

\subsubsection{A characterization of minimum closed cones}

A subset $Z$ of an arbitrary Euclidean vector space $I R^{k}$ is called minimum closed if for each two elements of $Z$, say $x^{1}, x^{2} \in Z$, also the coordinatewise minimum of $x^{1}$ and $x^{2}$ is contained in $Z$. Examples of cones which are minimum closed are given in lemma 4.1.

Lemma 4.1 A closed half-space $\mathcal{H}(y)=\left\{x \in I R^{k}: x \cdot y \geq 0\right\}$ has the minimum closedness property if and only if $y$ has at most one positive entry.

Proof: Suppose $y$ has at most one positive entry, say $y_{i}>0$ and $y_{j} \leq 0$ for all $1 \leq j \leq k, j \neq i$. Let $x^{1}, x^{2} \in \mathcal{*}(y)$. Without loss of generality assume that $x_{i}^{1} \leq x_{i}^{2}$. For $z=\left(\min \left(x_{j}^{1}, x_{j}^{2}\right)\right)_{1 \leq j \leq k}$ we have

$$
\begin{aligned}
z \cdot y & =z_{i} y_{i}-\sum_{j \neq i}\left|y_{j}\right| z_{j} \\
& =x_{i}^{1} y_{i}-\sum_{j \neq i}\left|y_{j}\right| z_{j} \\
& \geq x_{i}^{1} y_{i}-\sum_{j \neq i}\left|y_{j}\right| x_{j}^{1}=x \cdot y \geq 0 .
\end{aligned}
$$

Therefore, $z \in \mathcal{H}(y)$ which proves the minimum closedness of $\mathcal{H}(y)$.

Now suppose that $y$ has at least two positive entries, say $y_{i}>0$ and $y_{j}>0$. Let $x \in \mathcal{H}(y)$ be such that $x \cdot y=0$, and consider the two elements $x^{1}=x+y_{i} \chi^{\{j\}}-y_{j} \chi^{[i]}$ and $x^{2}=x+y_{j} \chi^{\{i\}}-y_{i} \chi^{\{j\}}$. Then

$$
x^{1} \cdot y=x \cdot y+y_{i} y_{j}-y_{j} y_{i}=x \cdot y=0
$$

and, similarly, $x^{2} \cdot y=0$. See also figure 4.1. Therefore, $x^{1}$ and $x^{2}$ are elements of $\mathcal{H}(y)$. The minimum of these two elements, say $z$, equals $x$ on all entries except $i$ and $j$, and $z_{i}=x_{i}-y_{j}$ and $z_{j}=x_{j}-y_{i}$. Then 


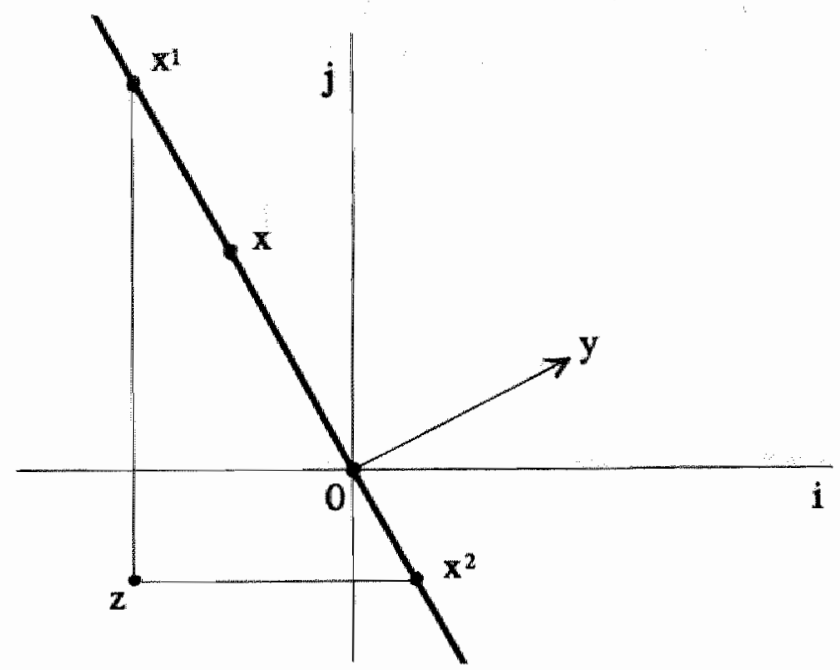

Figure 4.1: The relative positions of $x^{1}, x^{2}$ and $z$

$$
\begin{aligned}
z \cdot y & =x \cdot y-x_{i} y_{i}+\left(x_{i}-y_{j}\right) y_{i}-x_{j} y_{j}+\left(x_{j}-y_{i}\right) y_{j} \\
& =-2 y_{i} y_{j}<0
\end{aligned}
$$

i.e., $z \notin \mathcal{K}(y)$, implying that $\mathcal{K}(y)$ is not minimum closed.

Intersections of minimum closed cones are, again, minimum closed. Thus intersections of closed half-spaces with normal vectors having at most one positive entry are minimum closed. We conjecture that for closed cones the reverse also holds. We only prove here the following

Theorem 4.2 A polyhedral cone $Z \subseteq I R^{k}$ with non-empty interior is minimum closed if and only if there exists a finite set of normal vectors $Y \subset I R^{k}$, all of them having at most one positive entry, such that $Z=\cap_{y \in Y} H(y)$.

Proof: Let $Z$ be a polyhedral cone with non-empty interior. The validness of the 'if'-part has already been mentioned. Thus suppose $Z$ is minimum closed and let $Y$ be a set of normal vectors such that $Z=n_{y \in Y} H(y)$. Since $Z$ is polyhedral the set $Y$ may be chosen finite. Furthermore, let $Y$ be minimal, i.e., $Z \neq \cap_{y \in Y \backslash\left\{y^{\prime}\right\}} \mathcal{X}(y)$ for each $y^{\prime} \in Y$. Also, let $z$ be an element of the interior of $Z$ and $\epsilon>0$ such that the $\epsilon$-ball around $z$ is a subset of $Z$. Consider an arbitrary normal vector $y^{\prime} \in Y$. There is an $x \in I R^{k}$ such that $x \cdot y^{\prime}<0$ and $x \cdot y \geq 0$ for all $y \in Y \backslash\left\{y^{\prime}\right\}$. The element $x^{\prime}=x-\frac{x \cdot y^{\prime}}{z \cdot y^{\prime}} z$ fulfils $x^{\prime} \cdot y^{\prime}=x \cdot y^{\prime}-x \cdot y^{\prime}=0$ and $x^{\prime} \cdot y=x \cdot y-\frac{x \cdot y^{\prime}}{z \cdot y^{\prime}} \cdot y \cdot y>0$ since $x \cdot y \geq 0,-x \cdot y^{\prime}>0$, 
$z \cdot y^{\prime}>0$ and $z \cdot y>0$. Thus, there is a $\delta>0$ such that the intersection of the $\delta$-ball around $x^{\prime}$ and $K^{\prime}\left(y^{\prime}\right)$ is a subset of $Z$. The idea is now to use a similar reasoning as in the proof of lemma 4.1. Therefore, suppose $y^{\prime}$ has at least two positive entries, say $y_{i}^{\prime}>0$ and $y_{j}^{\prime}>0$ and let $\alpha$ denote the positive amount $\frac{\delta}{\left(v_{i}^{\prime}\right)^{2}+\left(y_{j}^{\prime}\right)^{2}}$. Consider the elements $x^{1}=x^{\prime}+\alpha\left(y_{i} \chi^{\{j\}}-y_{j} \chi^{\{i\}}\right)$ and $x^{2}=x^{\prime}+\alpha\left(y_{j} \chi^{\{i\}}-y_{i} \chi^{\{j\}}\right)$. Then $\left\|x^{1}-x^{\prime}\right\|=\delta,\left\|x^{2}-x^{\prime}\right\|=\delta$ and $x^{1} \cdot y^{\prime}=x^{2} \cdot y^{\prime}=0$, implying $x^{1}, x^{2} \in Z$. Similar as in the proof of lemma 4.1 we obtain that the minimum of $x^{1}$ and $x^{2}$ cannot be an element of $\mathcal{X}\left(y^{\prime}\right)$, implying $Z \mathbb{Z}\left(y^{\prime}\right)$; a contradiction. Therefore, $y^{\prime}$ has at most one positive entry. This proves the theorem.

\subsubsection{Minimum closed cones of games}

In the introduction we mentioned several minimum closed cones of games. That these cones are indeed minimum closed can be checked by considering the normals of the half-spaces corresponding to the inequalities which define the specific cone. The monotonicity conditions, for example, correspond to the closed half-spaces $\mathcal{H}(y)$ with normal vectors $y=\mathbb{1}^{S}-\mathbb{1}^{T}, T \subseteq S \subseteq$ $N$. These normals clearly fulfil the condition mentioned in lemma 4.1 and, therefore, the cone of monotonic games $\mathrm{MN}_{+}^{N}$ and each intersection of a minimum closed cone of games with $\mathbf{M N}_{+}^{N}$ is minimum closed.

The superadditivity conditions correspond to the normal vectors $y=$ $\mathbb{1}^{S \cup T}-\mathbf{1}^{S}-\mathbf{1}^{T}$, for disjoint coalitions $S, T \subseteq N$. All these normals have only one positive entry. Notice that the (totally) balanced vectors as defined on page 25 also possess this property. Furthermore, observe that the coalition corresponding to the positive entry contains each coalition for which the corresponding entry is negative. In fact this property holds in general for each cone of non-negative games containing the non-negative additive games. Before we prove this, we remark that from theorem 2.27, where we showed that each totally balanced game is the minimum of a finite collection of additive games, it follows that

Corollary 4.3 A minimum closed cone of games containing the (non-negative) additive games $\mathbf{A}^{N}$ ( $\mathbf{A}_{+}^{N}$ ) as a subset also contains all (non-negative) totally balanced games.

Theorem 4.4 Suppose that the cone $\mathrm{W}^{N} \subseteq \mathbf{G}_{+}^{N}$ encloses the cone $\mathbf{A}_{+}^{N}$. Then $\mathbf{W}^{N}$ is a minimum closed polyhedral cone if and only if $\mathbf{W}^{N}$ equals 
the intersection $\cap_{y \in Y} \nVdash(y)$ of a finite family of closed half-spaces with normal vectors $y \in Y \subset I R^{2^{N} \backslash(0)}$ having the property that there is a coalition $S$ with $y_{S}=1$ and for each other coalition with $y_{T} \neq 0$ we have $T \subset S$ and $y_{T}<0$.

\section{Proof :}

('only if'-part) The polyhedrality of the cone $Z$ implies that there is a finite set $Y$ of normal vectors such that $Z=n_{v \in Y} X(y) . W^{N}$ contains all non-negative totally balanced games and in particular the unanimity games. Since these games form a base for the game space (see corollary 3.27 ) it follows that $W^{N}$ has a non-empty interior. According to theorem 4.2 we may assume that each of the elements of $Y$ has at most one positive entry. Furthermore, since $Z \subseteq \mathrm{G}_{+}^{N}$ we may assume that $\mathbf{1}^{S} \in Y$ for each $S \subseteq N$, $S \neq \emptyset$. Also, each normal $y \in Y$ with non-positive entries may be deleted form $Y$ since for these normal vectors we have $Z \subseteq \mathbf{G}_{+}^{N}=\cap_{S \subseteq N, S \neq \emptyset} *\left(\mathbf{1}^{S}\right) \subseteq$ $K(y)$.

Now since all elements in $Y$ have exactly one positive entry, this entry may be considered to be normalized to 1 . For an arbitrary $y \in Y$ let $S \subseteq N$ be such that $y_{S}=1$. According to corollary 4.3 the totally balanced unanimity games $u^{T}$, with $T \subseteq N, T \neq \emptyset$, are contained in $Z$ and, therefore, $u^{T} \cdot y \geq 0$. Now for a coalition $S^{\prime}$ with $S^{\prime} \& S$ we have $u^{S^{\prime}} \cdot y=\sum_{T \supseteq S^{\prime} y_{T} \geq 0}$ which is a sum of non-positive entries. This implies that the summands have to be zero; in particular, $y_{S^{\prime}}=0$. This holds for each coalition $S^{\prime}$ not included in $S$ as a subset and this is exactly what was left to be proven. ('if'-part) Suppose that $\mathbf{W}^{N}=\cap_{v \in Y} H(y)$ with $Y$ as in the theorem. Thus $W^{N}$ is a polyhedral cone and according to our remark after lemma 4.1 it is minimum closed.

\section{Proper cones}

Let us call a cone $\mathbf{W}^{N} \subseteq \mathbf{G}^{N}$ of games proper if

- $\mathbf{W}^{N}$ is polyhedral;

- it fulfils the minimum closedness property, and

- it contains the dictator games.

The latter property implies that all non-negative additive games are contained in $\mathbf{W}^{N}$ and, thus, we may therefore assume that there exists a finite set of normal vectors with the properties described in theorem 4.4.

All mentioned minimum closed cones of games are polyhedral and contain the dictator games. 


\subsubsection{Essential coalitions and minimum covers}

With respect to a minimum closed cone $\mathbf{W}^{N} \subseteq \mathbf{G}_{+}^{N}$ the notion of essentiality of a non-empty coalition in a game is defined as follows: for a game $v \in \mathbf{W}^{N}$ a coalition $S$ is essential if there is an $\epsilon>0$ sufficiently small such that $v-\epsilon \mathbf{I}^{S}$ is still contained in $\mathbf{W}^{N}$.

essential coalitions of a superadditive game

In the case $W^{N}$ is the class of non-negative superadditive games the notion of essentiality of a coalition agrees with the one which is known in the literature (see Shapley [1971]): A coalition $S$ for a game $v \in \mathbf{S A}_{+}^{N}$ is essential if

$$
v(S)>0 \text { and } \quad v(S)>v(T)+v(S \backslash T) \text { for all } T \subset S, T \neq \emptyset .
$$

In a superadditive simple game the essential coalitions are exactly those coalitions with worth 1 and for which none of its subsets has worth 1 ; these coalitions are known as the minimal winning coalitions.

essential coalitions of a totally balanced game

Let us consider an essential coalition $S$ of a totally balanced game $v$, where the essentiality is considered with respect to the class $\mathbf{T B}_{+}^{N}$. Suppose that $v-\epsilon \mathbb{1}^{S}$ is still contained in $\mathbf{T B}_{+}^{N}$ for a particular positive scalar $\epsilon$. For a totally balanced vector $\lambda$ as defined in (1.22) we have $0 \leq \lambda \cdot\left(v-\epsilon \mathbb{1}^{S}\right)=$ $\lambda \cdot v-\epsilon \lambda_{S}$. Therefore, if $S$ is essential then

$$
0<\lambda \cdot v \text { for all totally balanced vectors } \lambda \text { with } \lambda_{S}>0 \text {. }
$$

Observe that also the reverse holds.

\section{Some regulte}

We remark here that in a proper cone $\mathbf{W}^{N}$ a game $v$ with no essential coalitions must equal the all-losing game. To show this, let $Y$ be as in theorem 4.4, and suppose there is a coalition $S$ with $v(S)>0$. We may assume that $v(T)=0$ for all $T \subset S$. Consider the scalar $0<\epsilon<v(S)$. If for a vector $y \in Y$ we have $y_{s}>0$, then

$$
\left(v-\epsilon \mathbb{1}^{S}\right) \cdot y=\sum_{T \subseteq S}\left(v-\epsilon \mathbb{1}^{S}\right)(T) y_{T}=(v(S)-\epsilon) y_{S}>0
$$

and if $y_{S} \leq 0$, then

$$
\left(v-\epsilon \mathbf{1}^{S}\right) \cdot y=v \cdot y-\epsilon \mathbb{1}^{S} \cdot y \geq v \cdot y \geq 0 .
$$


We conclude that $v-\epsilon \mathbf{1}^{S}$ is contained in $\mathbf{W}^{N}$, implying the essentiality of $S$; a contradiction.

The following result will be of significant help in the remaining part of this chapter.

Lemma 4.5 Let $\mathbf{W}^{N}$ be a proper cone of games and $v, w \in \mathbf{W}^{N}$. If for each essential coalition $S$ of $v$ we.have $v(S) \leq w(S)$ then $v(S) \leq w(S)$ holds for all coalitions, i.e., $v \leq w$.

Proof: Suppose on the contrary that there is a coalition $T$ " $\subseteq N$ with $v(S)>w(T)$. We may choose $T$ such that $v(S) \leq w(S)$ holds for all $S \subset T$. Of course, $T$ cannot be essential for $v$, i.e., $v-\epsilon \mathbf{I}^{T} \notin \mathbf{W}^{N}$ for all $\epsilon>0$. Let $Y$ be a finite set of normals such that $\mathbf{W}^{N}=\cap_{y \in Y} K(y)$ and such that the elements of $Y$ have the property mentioned in theorem 4.4 (note that $\mathrm{W}^{N}$ is proper). Let $y \in Y$ be chosen such that $v-\epsilon \mathbf{1}^{T} \notin \mathcal{X}(y)$ for all $\epsilon>0$ (the fact that $Y$ is finite makes this choice possible). Then from $v \in \mathcal{K}(y)$ it follows that $v \cdot y=0$. From $-\epsilon y_{T}=\left(v-\epsilon \mathbb{1}^{T}\right) \cdot y<0$ we obtain $y_{T}>0$. Now consider the inner product $w \cdot y=w(T)-\sum_{S \subset T}\left|y_{S}\right| w(S)$. Using $v(T)>w(T)$ and $v(S) \leq w(S)$ for all coalitions $T$ strictly included in $S$ we conclude that $w \cdot y$ is strictly majorized by $v(T)-\sum_{S \subset T}\left|y_{S}\right| v(S)=v \cdot y=0$; a contradiction since $w \cdot y \geq 0$ according to $w \in \mathbf{W}^{N} \subseteq \mathcal{K}(y)$. This proves the lemma.

The lemma in fact states that for a game $v$ in a proper cone $W^{N}$ one only needs to know the worths of the essential coalitions of a game $v$ in order to know the whole game $v$. This follows from

$$
v=\min \left(\left\{w \in \mathrm{W}^{N}: w(S) \geq v(S) \quad \text { for all } S \in \operatorname{Ess}\left(v, \mathrm{~W}^{N}\right)\right\}\right)
$$

where $\operatorname{Ess}\left(v, \mathbf{W}^{N}\right) \subseteq 2^{N} \backslash\{\emptyset\}$ denotes the set of essential coalitions of $v$ with respect to the cone $\mathbf{W}^{N}$. If we know less than the worths of all essential coalitions of a game then we cannot trace the whole game. For a more detailed discussion of the notion of essential coalitions with respect to $\mathrm{SA}_{+}^{N}$ the reader is referred to Derks [1987b]. An application of essential coalitions can be found in Derks and Kuipers [1991] where a special structure on the set of essential coalitions is discussed which implies the non-emptiness of the core.

\section{The minimum cover}

The above property (4.1) leads to the natural notion of a minimum cover. For a game $v$ the minimum cover, $v_{\min }$, with respect to a proper cone $\mathbf{W}^{N}$, is defined by

$$
v_{\min }=\min \left(\left\{w \in \mathbf{W}^{N}: w(S) \geq v(S) \text { for all } S \subseteq N\right\}\right.
$$


The minimum cover with respect to the superadditive games is called the superadditive cover. In a similar way the monotonic cover, and the (monotonic, totally) balanced cover are defined.

Especially the superadditive covers have been studied extensively in literature. For a direct application of superadditive covers we refer to the last section of the following chapter.

Let us list some results concerning essential coalitions and minimum covers.

Lemma 4.6 Let $\mathrm{W}^{N}$ be a minimum closed cone of non-negative monotonic games containing $\mathbf{A}_{+}^{N}$ as a subset. For a non-negative game $v$ and its minimum cover $v_{\min }$ (with respect to $\mathbf{W}^{N}$ ) we have, with $E=\{S \subseteq N: v(S)>$ o\},

i) if $\{S\}=E \cap 2^{T}$ for a coalition $T$ then $v_{\min }(T)=v(S)$;

ii) $v_{\min }(T)=0$ if and only if $E \cap 2^{T}=0$;

iii) $E s s\left(v_{\min }, \mathbf{W}^{N}\right) \subseteq E$;

iv) if $E$ is proper then $E s s\left(u_{\min }, \mathbf{W}^{N}\right)=E$.

Here, a set $B$ of coalitions is said to be proper if $S, T \in E, S \subseteq T$, implies $S=T$ (cf. Shapley [1967]).

Proof:

Proof of i).

Consider the additive game $u$ corresponding to the allocation $v(S) \chi^{\{j\}}+$ $M \chi^{N \backslash T}$, with player $j \in S$ arbitrary and scalar $M$ chosen such that $u$ majorizes $v$ (this is possible since $v\left(S^{\prime}\right)=0$ for all $S^{\prime} \in 2^{T} \backslash\{S\}$ ). Since $u \in \mathbf{W}^{N}$ we must have $v_{\min } \leq u$ and in particular $v_{\min }(T) \leq u(T)=v(S)$. Since $v_{\min }$ is monotonic we obtain $v_{\min }(T) \geq v_{\min }(S) \geq v(S)$. Thus equality $v_{\min }(T)=v(S)$ holds.

Proof of ii).

If $E \cap 2^{T}$ is non-empty, say $S \in E \cap 2^{T}$, then $0<v(S) \leq v_{\min }(S) \leq v_{\min }(T)$ and if $E \cap 2^{T}$ is empty then the additive game $u$ corresponding to the allocation $M \chi^{N \backslash T}$, with $M$ such that $u \geq v$, yields $0=v(T) \leq v_{\min }(T) \leq$ $M \chi^{N \backslash T}(T)=0$, i.e., $v_{\min }(T)=0$.

Proof of iii).

For an essential coalition $T$ of $v_{\min }$ we have $v_{\min }-\epsilon \mathbf{1}^{T} \in \mathbf{W}^{N}$ for $\epsilon>0$ sufficiently small. Observe that the inequality $\left(v_{\min }-\epsilon \mathbf{1}^{T}\right)(T) \geq v(T)$ contradicts the definition of $v_{\min }$. Therefore, $0 \leq\left(v_{\min }-\epsilon \mathbb{1}^{T}\right)(T)<v(T)$ which proves that $T \in E$. 
Proof of iv).

Let $i$ be an arbitrary player in a coalition $T \in E$, and let $\epsilon>0$ be such that $\epsilon<v(T)$. Since $E$ is assumed to be proper, we have $\{T\}=E \cap 2^{T}$ and, thus, $v_{\min }(T)=v(T)$ and $v_{\min }(S)=0$ for all $S \subset T$ according to i) and ii). Consider the additive game $u$ corresponding to the allocation $(v(T)-\epsilon) \chi^{\{i\}}+M \chi^{N \backslash T}$, with $M$ sufficiently large, say $M=\sum_{S \subseteq N} v_{\min }(S)$. The minimum of $v_{\min }$ and $u$ equals $v_{\min }-\epsilon \mathbb{1}^{T}$ which is therefore an element of $\mathbf{W}^{N}$, implying the essentiality of the coalition $T$ for $v_{\min }$. Together with iii) we conclude that $\operatorname{Ess}\left(v, \mathbf{W}^{N}\right)=E$.

\subsubsection{Decomposability}

Recall that the subgame of a game $v$ with respect to a coalition $S$ is denoted by $v_{\mid S}$ and is defined by $v_{\mid S}(T)=v(S \cap T)$ for each $T \subseteq N$.

Lemma 4.7 Let $\mathbf{W}^{N}$ be a proper cone of monotonic games and $v \in \mathbf{W}^{N}$. If the subgame $v_{\mid S}$ is an element of $\mathrm{W}^{N}$ then

$$
v_{\mid S}=\min \left(\left\{w \in \mathbf{W}^{N}: v(T) \leq w(T) \text { for all } T \subseteq S\right\}\right)
$$

Proof: Let $u$ denote the game $\min \left(\left\{w \in \mathbf{W}^{N}: v(T) \leq w(T)\right.\right.$ for all $T \subseteq$ $S\})$. The monotonicity of $u$ implies

$$
u(T) \geq u(T \cap S) \geq v(T \cap S)=v_{\mid S}(T) \quad \text { for each } T \subseteq N .
$$

Now if $v_{\mid S} \in \mathbf{W}^{N}$ then by definition we have $u \leq v_{\mid S}$, implying the required equality.

Notice that the subgames of a (non-negative and) superadditive or totally balanced game are also (non-negative and) superadditive or totally balanced. For a (monotonic) balanced game, however, a subgame may not be balanced.

A minimum closed cone $\mathrm{W}^{N}$ is called subgame closed if for each game $v \in \mathbf{W}^{N}$ all its subgames are also elements of $\mathbf{W}^{N}$.

\section{Decomposable games}

A game $v \in \mathbf{W}^{N}$, with $\mathbf{W}^{N}$ minimum closed and subgame closed, is called decomposable if there exists a coalition $S$ such that

$$
v_{\mid S} \neq 0, \quad v_{\mid N \backslash S} \neq 0, \text { and } v=v_{\mid S}+v_{\mid N \backslash S} .
$$


Decomposability with respect to the class $\mathbf{S A} \mathbf{A}^{N}$ has been considered first in Shapley [1971] where decomposable convex games have been characterized as those games for which the grand coalition is essential with respect to $\mathbf{S A}^{N}$.

Of course decomposable games cannot be extreme. Non-decomposable games may not be extreme either. Examples will be given. First, some elementary results are formulated.

A set $E$ of coalitions of $N$ is said to be decomposable if there is a coalition $S \subset N$ such that

$$
E \cap 2^{S} \neq \emptyset, \quad E \cap 2^{N \backslash S} \neq \emptyset \text { and } E=\left(E \cap 2^{S}\right) \cup\left(E \cap 2^{N \backslash S}\right)
$$

thus $E$ consists of coalitions which are included in either $S$ or the complement of $S$.

Lemma 4.8 Let $W^{N}$ be a proper and aubgame closed cone of non-negative superadditive games. A game $v \in \mathbf{W}^{N}$ is decomposable if and only if the set $\operatorname{Ess}\left(v, \mathbf{W}^{N}\right)$ of essential coalitions is decomposable.

Proof: Let $v \in \mathbf{W}^{N}$ be decomposable and coalition $S$ be as in (4.2). For an essential coalition $T$ of $v$, say $v-\epsilon \mathbf{1}^{T} \in \mathbf{W}^{N}$ with $\epsilon>0$ sufficiently small, we suppose for the moment that $T \notin S$ and $T \nsubseteq N \backslash S$. Then

$$
\begin{aligned}
\left(v-\epsilon \mathbf{1}^{T}\right)(T) & =v(T)-\epsilon<v(T) \\
& =v_{\mid S}(T)+v_{\mid N \backslash S}(T) \\
& =v(S \cap T)+v(N \backslash S \cap T) \\
& =\left(v-\epsilon \mathbb{1}^{T}\right)(S \cap T)+\left(v-\epsilon \mathbf{1}^{T}\right)(N \backslash S \cap T) .
\end{aligned}
$$

This is contradictory to the superadditivity of $v-\epsilon \mathbf{1}^{T}$ and we conclude therefore that an essential coalition of $v$ is either a subset of $S$ or of $N \backslash S$. Of course, we have $\operatorname{Ess}\left(v_{\mid S}, \mathbf{W}^{N}\right) \subseteq \operatorname{Ess}\left(v, \mathbf{W}^{N}\right)$, and according to lemma 4.7 the set $\operatorname{Ess}\left(v_{\mid S}, \mathbf{W}^{N}\right)$ is contained in $2^{S}$; therefore, $\operatorname{Ess}\left(v, \mathbf{W}^{N}\right) \cap 2^{S}=$ yields $v_{\mid S}=\emptyset$, implying $v_{\mid S}=0$, which is excluded. Therefore, $\operatorname{Ess}\left(v, \mathbf{W}^{N}\right) \cap 2^{S} \neq \emptyset$ and, by the same reasoning, also $\operatorname{Ess}\left(v, \mathbf{W}^{N}\right) \cap 2^{N \backslash S} \neq \emptyset$. We conclude that Ess $\left(v, \mathbf{W}^{N}\right)$ is decomposable.

Now let $\operatorname{Ess}\left(v, \mathbf{W}^{N}\right)$ be decomposable with $v \in \mathbf{W}^{N}$ and coalition $S$ as in (4.3) where $\operatorname{Ess}\left(v, \mathbf{W}^{N}\right)$ is taken as $E$. It follows that $v$ equals the game $v_{\mid S}+v_{\mid N \backslash S}$ on its essential coalitions, implying $v \leq v_{\mid S}+v_{\mid N \backslash S}$ according to lemma 4.5. Now since $v$ is superadditive we must have

$$
\begin{aligned}
v(T) & =v((T \cap S) \cup(T \cap N \backslash S)) \\
& \geq v(T \cap S)+v(T \cap N \backslash S) \\
& =v_{\mid S}(T)+v_{\mid N \backslash S}(T)
\end{aligned}
$$


for each coalition $T \subseteq N$. Therefore, equality $v=v_{\mid s}+v_{\mid N \backslash s}$ holds. Now $v_{\mid s} \neq 0$ and $v_{\mid N \backslash S} \neq 0$ since $\emptyset \neq \operatorname{Ess}\left(v, \mathbf{W}^{N}\right) \cap 2^{S} \subseteq \operatorname{Ess}\left(v \mid s, \mathbf{W}^{N}\right)$ and also $\emptyset \neq \operatorname{Ess}\left(v, \mathbf{W}^{N}\right) \cap 2^{N \backslash S} \subseteq \operatorname{Ess}\left(v_{\mid N \backslash s}, \mathbf{W}^{N}\right)$.

\section{Examples}

The assumption that the cone $W^{N}$ is a subset of $\mathbf{S A}_{+}^{N}$ in lemma 4.8 cannot be weakened to the cone $\mathbf{M N}_{+}^{N}$ of non-negative monotionic games. The two-person game $v$ and its subgames $v_{\mid\{1\}}$ and $v_{\{\{2\}}$

\begin{tabular}{c|ccc}
$S$ & $\{1\}$ & $\{2\}$ & $\{1,2\}$ \\
\hline$v$ & 1 & 1 & 1 \\
$v_{\mid\{1\}}$ & 1 & 0 & 1 \\
$v_{\mid\{2\}}$ & 0 & 1 & 1
\end{tabular}

do not fulfil $v=v_{\mid\{1\}}+v_{\mid\{2\}}$ although the set $\operatorname{Ess}\left(v, \mathbf{M N}_{+}^{N}\right)=\{\{1\},\{2\}\}$ is decomposable.

In the case

\begin{tabular}{c|ccc}
$S$ & $\{1\}$ & $\{2\}$ & $\{1,2\}$ \\
\hline$v$ & 1 & 1 & 2 \\
$v_{\mid\{1\}}$ & 1 & 0 & 1 \\
$v_{\mid\{2\}}$ & 0 & 1 & 1
\end{tabular}

we however have $v=v_{\mid\{1\}}+v_{\mid\{2\}}$ although $\operatorname{Ess}\left(v, \mathbf{M N}_{+}^{N}\right)=\{\{1\},\{2\},\{1,2\}\}$ is non-decomposable.

The following example shows that the non-decomposability of the set of essential coalitions not necessarily implies the extremality property. Consider the three-person superadditive games $v, w^{1}$ and $w^{2}$ :

\begin{tabular}{c|cccc}
$S$ & $\{1,2\}$ & $\{1,3\}$ & $\{2,3\}$ & $N$ \\
\hline$v$ & 1 & 1 & 1 & 2 \\
$w^{1}$ & 1 & 1 & 0 & 1 \\
$w^{2}$ & 0 & 0 & 1 & 1
\end{tabular}

Then $v$ equals $w^{1}+w^{2}$ which are no multiples of $v$. Thus $v$ is not extreme in $\mathbf{S A}_{+}^{N}$. The set of essential coalitions contains the two-person coalitions and $N$, which is non-decomposable. 


\subsubsection{Extreme games}

In the previous examples we saw that the non-decomposability of a game in a proper cone of non-negative monotonic games is a necessary but not a sufficient condition for the extremality property. The next theorem introduces sufficient conditions in order to obtain extremality.

Theorem 4.9 Let $\mathbf{W}^{N} \subseteq \mathrm{MN}_{+}^{N}$ be a proper cone of games with the subgame closedness property. Let $\theta \in \mathbf{W}^{N}$ be such that there is a sequence of essential coalitions of $v$, say $T_{1}, \ldots, T_{R}$, with the properties

1) $\operatorname{Ess}\left(v, \mathbf{W}^{N}\right)=\left\{T_{r}: 1 \leq r \leq R\right\}$, i.e., each essential coalition of $v$ is included in the sequence;

2) the subgames $v_{\| T_{r} \cup T_{r+1}}, 1 \leq r<R$, are extreme in $\mathrm{W}^{N}$.

Then $v$ is extreme.

Proof: Let $w^{1}, w^{2} \in \mathbf{W}^{N}$ be such that $v=w^{1}+w^{2}$. For each $1 \leq r<R$ the game $v_{\mid T_{r} \cup T_{r+1}}$ equals $w_{\mid T_{r} \cup T_{r+1}}^{1}+w_{\mid T_{r} \cup T_{r+1}}^{2}$; using the extremality of $v_{\mid T_{r}, U T_{r+\mathbb{H}}}$ we conclude that there exist non-negative scalars $\alpha_{r}^{1}, \alpha_{r}^{2}$ such that $w_{\mid T_{r} \cup T_{r+1}}^{i}=\alpha_{r}^{i} v_{\mid T_{r} \cup T_{r+1}}$, with $i=1,2$. Then

$$
\alpha_{r}^{i} v\left(T_{r+1}\right)=\alpha_{r}^{i} v_{\mid T_{r} \cup T_{r+1}}\left(T_{r+1}\right)=w_{\mid T_{r} \cup T_{r+1}}^{i}\left(T_{r+1}\right)=w^{i}\left(T_{r+1}\right)
$$

and, similarly, $\alpha_{r+1}^{i} v\left(T_{r+1}\right)=w^{i}\left(T_{r+1}\right)$, implying $\alpha_{r}^{i}=\alpha_{r+1}^{i}, 1 \leq r<R$, $i=1,2$ (the essentiality of $T_{r+1}$ implies that $v\left(T_{r+1}\right)>0$ ). Let $\alpha^{i}=\alpha_{r}^{i}$, $1 \leq r<R, i=1,2$. We have $\alpha^{1}+\alpha^{2}=1$ since

$$
v\left(T_{1}\right)=w^{1}\left(T_{1}\right)+w^{2}\left(T_{1}\right)=\alpha^{1} v\left(T_{1}\right)+\alpha^{2} v\left(T_{1}\right)=\left(\alpha^{1}+\alpha^{2}\right) v\left(T_{1}\right) .
$$

Using $\operatorname{Ess}\left(\alpha^{i} v, \mathbf{W}^{N}\right)=\emptyset$ if $\alpha^{i}=0$ and $\operatorname{Ess}\left(\alpha^{i} v, \mathbf{W}^{N}\right)=\operatorname{Ess}\left(v, \mathbf{W}^{N}\right)=\left\{T_{r}\right.$ : $1 \leq r \leq R\}$ if $\alpha^{i}>0$, and $\alpha^{i} v\left(T_{r}\right)=w^{i}\left(T_{r}\right)$ for all $1 \leq r \leq R, i=1,2$, we conclude that $\alpha^{1} v \leq w^{1}$ and $\alpha^{2} v \leq w^{2}$ (see lemma 4.5). Therefore,

$$
w^{1}+w^{2}=v=\alpha^{1} v+\alpha^{2} v \leq w^{1}+w^{2}
$$

i.e., $w^{1}$ and $w^{2}$ are non-negative multiples of $v$. Hence, $v$ is extreme.

In order to apply the above theorem we must know at least some of the extreme elements of $\mathbf{W}^{N}$. Since $\mathbf{W}^{N}$ is a subset of $\mathbf{G}_{+}^{N}$, the simple games in $\mathbf{W}^{N}$ are extreme (see lemma 1.20). Thus a game $v \in \mathbf{W}^{N}$, where $\mathbf{W}^{N}$ 
fulfils the conditions in theorem 4.9 , is extreme if there exists a sequence of essential coalitions $T_{1}, \ldots, T_{R}$ in which each essential coalition is included and for which $v_{\mid T_{r} \cup T_{r+1}}$ is simple, $1 \leq r<R$.

\section{Uniform games}

The fact that $v_{\mid T_{r} \cup T_{r+1}}$ is simple for each $1 \leq r<R$ in the observation above implies that the worth of each essential coalition in $v$ equals 1 . This gives rise to the following notion. A game $v \in \mathbf{W}^{N} \subseteq \mathbf{G}_{+}^{N}$, with $\mathbf{W}^{N}$ minimum closed, is called uniform if $v(S)=1$ for each $S \in \operatorname{Ess}\left(v, \mathbf{W}^{N}\right)$. Uniform games may be seen as a generalization of the simple games since each simple game in $\mathbf{W}^{N}$ is uniform.

\subsection{The cone of non-negative superadditive ga- mes}

\subsubsection{Extreme superadditive games}

In Derks [1987b] it is proved that among the uniform games in $\mathbf{S A}_{+}^{N}$ the extreme games are exactly the non-decomposable games. In this section we will prove this result with the help of theorem 4.9. First some preliminary results are needed.

Lemma 4.10 Let $E \subseteq 2^{N}$ be a non-empty, non-decomposable, proper set of coalitions. Then there exists a sequence $Q=\left(T_{1}, \ldots, T_{R}\right)$ of elements of $E$ such that

i) $E=\left\{T_{r}: 1 \leq r \leq R\right\}$;

ii) the union of each two adjacent elements of $Q$ does not contain two disjoint elements of $E$, i.e.,

if $S, T \subseteq T_{r} \cup T_{r+1}$ for an $r, 1 \leq r<R$ and $S, T \in E$, then $S \cap T \neq \emptyset$.

Proof: In the following we shall construct a sequence which has the two desired properties. Note that for a sequence for which ii) holds the intersection of each two adjacent elements is non-empty.

Let $Q=\left(T_{1}, \ldots, T_{R}\right)$ be an arbitrary sequence of elements of $E$ for which we have

$$
T_{r} \cap T_{r+1} \neq \emptyset, \quad 1 \leq r<R,
$$

and suppose that there is an element of $E$ which does not occur in $Q$. Let $T$ be the union of the sets $T_{1}, \ldots, T_{R}$. Consider the following two cases: 
a) $E \cap 2^{N \backslash T}=\emptyset$

Then each element $S^{\prime}$ of $E \backslash\left\{T_{r}: 1 \leq r \leq R\right\}$ fulfils $S^{\prime} \subseteq T$.

b) $E \cap 2^{N \backslash T} \neq 0$.

$\left(E \cap 2^{T}\right) \cup\left(E \cap 2^{N \backslash T}\right) \neq E$ since $E$ is not decomposable by assumption. Therefore, there is an element, say $S^{\prime}$, of $E$ such that $S^{\prime} \cap T \neq \emptyset$ and also $S^{\prime} \cap N \backslash T \neq \emptyset$.

In both cases it is possible to choose an element $S^{\prime}$ of $E \backslash\left\{T_{r}: 1 \leq r \leq R\right\}$ such that $S^{\prime} \cap T \neq \emptyset$. Of course, there is a coalition $T_{r}, 1 \leq r \leq R$, such that $S^{\prime} \cap T_{r} \neq \emptyset$. Now the sequence $Q^{\prime}=\left(T_{1}, \ldots, T_{r}, S^{\prime}, T_{r}, \ldots, T_{R}\right)$ fulfils (4.4) also and it contains one extra element of $E$.

In this way one constructs a sequence of elements of $E$ in which each element of $E$ occurs at least once, thus fulfilling i), and for which (4.4) holds.

Now suppose there is an index $r$ and disjoint elements $S, T \in E$ such that $S \cup T \subseteq T_{r} \cup T_{r+1}$. If $S \cap T_{r}=0$ then $S \subseteq T_{r+1}$. Since $E$ is proper we must have $S=T_{r+1}$, implying $S \cap T_{r} \neq \emptyset$ according to (4.4); a contradiction. We conclude that $S \cap T_{r} \neq \emptyset$ and, similarly, $S \cap T_{r+1} \neq \emptyset$.

The sequence $Q^{1}=\left(T_{1}, \ldots, T_{r}, S, T_{r+1}, \ldots, T_{R}\right)$ therefore fulfils i) and (4.4). The number of players in $T_{r} \cup S$ is strictly smaller than in $T_{r} \cup T_{r+1}$. To show this suppose $\left|T_{r} \cup S\right| \geq\left|T_{r} \cup T_{r+1}\right|$. Then we must have $T_{r+1} \backslash T_{r} \subseteq S$. Using $S \cap T=$ we obtain $T \subseteq T_{r}$, implying $T=T_{r}$ ( $E$ is proper) and thus $S \cap T \neq \emptyset$; a contradiction.

Therefore, $\left|T_{r} \cup S\right|<\left|T_{r} \cup T_{r+1}\right|$ and, similarly, $\left|S \cup T_{r+1}\right|<\left|T_{r} \cup T_{r+1}\right|$. From this property we conclude that we cannot insert a coalition $S$ infinitely many times as described above and we eventually obtain a sequence for which not only i) but also ii) must hold.

The following lemma characterizes the simple games among the uniform superadditive games.

Lemma 4.11 Let $v \in \mathbf{S A}_{+}^{N} \backslash\{0\}$ be a uniform game. Then $v$ is simple if and only if each two essential coalitions have a non-empty intersection.

Proof :

('only if'-part) The essential coalitions of $v$ have worths 1 . If two of these coalitions have an empty intersection then the union has a worth of at least 2 , which is impossible. 
('if'-part) Let $w$ be the simple game defined by

$$
w(S)= \begin{cases}1 & \text { if } \operatorname{Ess}\left(u, \mathbf{S A}_{+}^{N}\right) \cap 2^{s} \neq \emptyset \\ 0 & \text { otherwise. }\end{cases}
$$

Then $w$ is a monotonic game with $w \leq v$. Suppose $w$ is not superadditive, say $w(S \cup T)<w(S)+w(T)$ for two disjoint coalitions $S$ and $T$. The monotonicity of the simple game $w$ implies $1=w(S \cup T)=w(S)=w(T)$. Therefore $\operatorname{Ess}\left(v, \mathbf{S A}_{+}^{N}\right) \cap 2^{S} \neq 0$ and $\operatorname{Ess}\left(v, \mathbf{S A}_{+}^{N}\right) \cap 2^{T} \neq 0$, implying that two disjoint essential coalitions in $\operatorname{Ess}\left(v, \mathbf{S A}_{+}^{N}\right)$ exist; a contradiction. We conclude that $w$ must be superadditive.

The equalities $w(S)=v(S)$ for $S \in \operatorname{Ess}\left(v, S_{+}^{N}\right)$ now implies $w \geq v$ according to lemma 4.5. We mentioned already that $w \leq v$ and, thus, $v=w$.

We are now able to show the validity of our main result.

Theorem 4.12 Let $v \in \mathbf{S A}_{+}^{N}$ be uniform. Then $v$ is extreme in $\mathbf{S A}_{+}^{N}$ if and only if $\operatorname{Ess}\left(v, \mathbf{S A}_{+}^{N}\right)$ is non-decomposable.

Proof: The 'only if'-part is a direct consequence of lemma 4.8.

Thus suppose that the set $\operatorname{Ess}\left(v, \mathbf{S A}_{+}^{N}\right)$ is non-decomposable. According to lemma 4.10 there exists a sequence $T_{1}, \ldots, T_{R}$ of essential coalitions for which i) and ii) of lemma 4.10 hold. Property ii) and lemma 4.11 imply that the subgames $v_{\mid T_{r} \cup T_{r+1}}, 1 \leq r<R$, are simple. The conditions in theorem 4.9 are therefore satisfied, implying the extremality of $v$.

\section{Examples}

Well-known examples of uniform superadditive games, besides the simple games, are the glove games, defined in subsection 1.2 ( $v$ is a glove game if $v(S)=\min (|P \cap S|,|Q \cap S|), S \subseteq N$, where $P$ and $Q$ are two coalitions with $P \cup Q=N)$. The glove game is obviously superadditive. The essential coalitions are the one-person coalitions of the players in the intersection $P \cap Q$ and all two-person coalitions with one player from $P \backslash Q$ and the other from $Q \backslash P$. The worths of the essential coalitions all equal 1 . Therefore, $v$ is uniform. Furthermore, Ess $\left(v, \mathbf{S A}_{+}^{N}\right)$ is decomposable if and only if $P \cap Q \neq \emptyset$. Using theorem 4.12 we conclude that the glove game is extreme in $\mathrm{SA}_{+}^{N}$ if and only if $P \cap Q=\emptyset$.

Notice that the glove game, with $P \cap Q=0$, has a non-empty core: letting $P$ be such that $|P| \leq|Q|$, the allocation $\chi^{P}$, distributing 1 to the players in 
$P$ and 0 to the players in $Q$, denotes a core allocation. Furthermore, each subgame of this glove game is again a glove game of the above type, and, thus, with a non-empty core. Using the Bondareva-Shapley correspondence we conclude that the glove games with $P \cap Q=\emptyset$ are totally balanced. The fact that these games are extreme in the class $\mathbf{S A}_{+}^{N}$ of non-negative superadditive games implies that these games are also extreme in the smaller class $\mathbf{T B}_{+}^{N}$ of non-negative totally balanced games. This shows that $\mathbf{T B}_{+}^{N}$ possesses non-simple extreme games.

Another example of a uniform superadditive game is the game $v$ on the player set $N$ defined by

$$
v(S)=\left\{\begin{array}{cl}
\frac{1}{2}|S| & \text { if }|S| \text { is even, } \\
\frac{1}{2}(|S|-1) & \text { if }|S| \text { is odd. }
\end{array} \quad \text { for each coalition } \mathrm{S},\right.
$$

i.e., the worth of a coalition $S$ equals the maximum amount of disjoint pairs of players in $S$. The set $\operatorname{Ess}\left(u, \mathbf{S A}_{+}^{N}\right)$ consists of all two-person coalitions and is, therefore, non-decomposable. The worths of the essential coalitions all equal 1. Therefore, $v$ is uniform and extreme in $\mathbf{S A}_{+}^{N}$. A similar game has been studied in Shubik [1971] where the worth of a coalition $S$ is computed as the maximum amount of disjoint four-person coalitions with players in $S$. Again this game is uniform with essential coalitions the four-person coalitions.

\section{Counterexamples}

Unfortunately, theorem 4.9 does not characterize all extreme games in $\mathbf{S A}_{+}^{N}$. For example, in Spinetto [1971] all four-person extreme games are enumerated (symetric cases are omitted) among which the game $v$ defined by

\begin{tabular}{ccccccccc}
$\{1,2\}$ & $\{1,3\}$ & $\{2,3\}$ & $\{3,4\}$ & $\{1,2,3\}$ & $\{1,2,4\}$ & $\{1,3,4\}$ & $\{2,3,4\}$ & $N$ \\
\hline$\frac{1}{2}$ & $\frac{1}{2}$ & $\frac{1}{2}$ & $\frac{1}{2}$ & $\frac{1}{2}$ & 1 & 1 & $\frac{1}{2}$ & 1
\end{tabular}

(as usual the not mentioned coalitions have worths 0 ). This game is not uniform or a multiple of a uniform game since $\{1,2\}$ and $\{1,2,4\}$ are essential coalitions with different non-zero worths. Now let us try to find a sequence $T_{1}, \ldots, T_{R}$, using the essential coalitions $\{1,2\},\{1,3\},\{2,3\},\{3,4\},\{1,2,4\}$ and $\{1,3,4\}$, such that $v_{\mid T_{r} \cup T_{r+1}}$ is extreme, $1 \leq r<R$. Excluding the case $T_{r} \cup T_{r+1}=N$ we observe that coalition $\{1,2,4\}$ can only have $\{1,2\}$ as its 
neighbour. Now consider index $r$ for which $T_{r}=\{1,2,4\}$ and $T_{r+1}=\{1,2\}$ (or vice versa). Then $v_{\mid T_{r} \cup T_{r+1}}=v_{\mid\{1,2,4\}}$ is the game

\begin{tabular}{c|cccc}
$S$ & $\{1,2\}$ & $\{1,2,3\}$ & $\{1,2,4\}$ & $N$ \\
\hline$v_{\mid\{1,2,4\}}$ & $\frac{1}{2}$ & $\frac{1}{2}$ & 1 & 1
\end{tabular}

This game is, obviously, not extreme. We conclude that theorem 4.9 cannot be reversed in case $\mathbf{W}^{N}$ is the class of non-negative superadditive games and if we do not permit the trivial cases in which $T_{r} \cup T_{r+1}=N$ for an index $1 \leq r<R$.

This example shows that the extremality of the subgames with respect to the essential coalitions not necessarily implies the extremality of $v$.

Also the extremality of the non-trivial subgames $v_{\mid S}$ with respect to the essential coalitions $S \in \operatorname{Ess}\left(v, \mathbf{S A}_{+}^{N}\right)$, and the non-decomposability of $\operatorname{Ess}\left(v, \mathbf{S A}_{+}^{N}\right)$ are not sufficient in order to imply the extremality of the game $v$ itself. An example is the latter monotonic two-person game $v$ on page 135. Its two non-trivial subgames are extreme in $\mathbf{M N}_{+}^{N}, \operatorname{Ess}\left(v, \mathbf{M N}_{+}^{N}\right)$ is non-decomposable, yet $v$ is not extreme in $\mathbf{M N}_{+}^{N}$.

\subsubsection{A survey of results in the literature concerning ex- treme superadditive games}

Together with the introduction of the game model used here also the study on the subject of extreme superadditive games started in Von Neumann and Morgenstern [1944]. The theoretical model which gave rise to the definition. of a game imposed the superadditivity and the constant sum property. A garme $v$ is said to have the constant sum property if

$$
v(S)+v(N \backslash S)=v(N) \quad \text { for each coalition } S .
$$

The first result on extreme games therefore concerns the set

$$
\mathbf{L}^{N}=\left\{v \in \mathbf{S A}_{01}^{N}: v(S)+v(N \backslash S)=v(N) \text { for each } S \subseteq N\right\} .
$$

$\mathbf{L}^{N}$ is compact and convex and Von Neumann and Morgenstern showed that its extreme games of $\mathrm{L}^{N}$, with $|N|=4$, are exactly the simple games in $\mathrm{L}^{N}$.

The question was raised whether this was generally true. A negative answer was given in Gurk [1959] by characterizing the extreme games of $L^{N}$ with $|N|=5$. First Gurk noticed that a simple game in $\mathbf{L}^{N}$ is extreme 
(see also lemma 1.20). Then he considered three-valued games. For 0,1normalized constant sum games these worths are, of course, $0, \frac{1}{2}$ and 1 . Furthermore he introduced the following notion:

Let $v$ be an element of $\mathrm{L}^{N}$. A sequence $\left(T_{1}, \ldots, T_{2}\right)$ of coalitions is called a $K$-chain if
i) $T_{1}=T_{2 R}$,
ii) $T_{r} \cap T_{r+1}=\quad$ for each $r: 1 \leq r<2 R$, and
iii) $v\left(T_{r}\right)+v\left(T_{r+1}\right)=1$ for each $r: 1 \leq r<2 R$.

For a $K$-chain $\left(T_{1}, \ldots, T_{2 R}\right)$ we obviously have $v\left(T_{1}\right)=v\left(T_{2 r+1}\right)$ and $v\left(T_{2 R}\right)=$ $v\left(T_{2 r}\right)$ for each $r: 1 \leq r<2 R$. The equality $T_{1}=T_{2 R}$ now implies that the coalitions in a $K$-chain all have the same worths in the game $v$. Because of iii) we thus have

$$
v\left(T_{1}\right)=v\left(T_{2}\right)=\ldots=v\left(T_{2 R}\right)=\frac{1}{2} .
$$

Therefore, a $K$-chain for a game is a finite circular sequence of disjoint coalitions all having worths $\frac{1}{2}$.

Theorem 4.13 (Gurk) Let $v \in \mathbf{L}^{N}$ be a three-valued game. If for each coalition $S$ with $v(S)=\frac{1}{2}$ there exists a $K$-chain starting with $S$ then $v$ is extreme.

The $K$-chain property, as formulated in theorem 4.13 , is also sufficient in the case of five-person games.

Theorem 4.14 (Gurk) A game $v$ in $\mathbf{L}^{N}$, with $|N|=5$, is extreme if and only if for each coalition $S$ with worth unequal to $O$ or 1 there exists a $K$ chain starting at $S$.

Theorem 4.14 obviously implies that the extreme elements of $L^{N}$, with $|N|=5$, are three-valued games or simple games. Apart from the theorems above Gurk gave also an example of an extreme game in $\mathbf{L}^{N}$, with $|N| \geq 6$, which assigns more than three different worths to the coalitions (the extreme 'pair' game $v$ defined on page 140 has the constant sum property whenever there are an odd number of players; the worths in $v$ are $\left.0,1, \ldots, \frac{1}{2}(|N|-1)\right)$.

In Griesmer [1959] the reverse of theorem 4.13 is shown.

Theorem 4.15 (Griesmer) Let $v$ be a three-valued game of $\mathrm{L}^{N}$. If $v$ is extreme in $\mathbf{L}^{N}$, then for each coalition $S$ with worth $\frac{1}{2}$ there exists a $K$ chain starting at $S$. 
The theorems above can also be used in the characterization of the three-

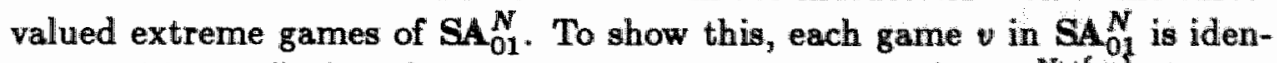
tified with an $(|N|+1)$-person constant sum game $v^{\prime} \in \mathbf{L}^{N \cup\{m\}}$ defined by

$$
v^{\prime}(S)=\left\{\begin{array}{cc}
v(S) & \text { if } m \notin S \\
1-v(N \backslash(S \backslash\{m\})) & \text { if } m \in S
\end{array} \quad \text { for each } S \subseteq N \cup\{m\}\right.
$$

(where $m$ is assumed not to be a member of $N$ ). In this context player $m$ is called the fictitious player.

Theorem 4.16 (Spinetto) A game $v$ of $\mathbf{S A}_{01}^{N}$ is extreme if and only if $v^{\prime}$ is extreme in $\mathrm{L}^{N \cup\{m\}}$.

By using the results in Gurk [1959] on the five-person constant sum games and applying theorem 4.16 Spinetto was able to list the four-person extreme superadditive games. Of course, these games are three-valued or simple.

In Rosenmüller [1977] a completely different approach to the problem of characterizing (the) extreme games of $\mathbf{S A}_{+}^{N}$ is proposed. The aim is to represent a game in $\mathbf{S A}_{+}^{N}$ in such a way that the extremality property can be translated into properties of the representation. This approach has been applied succesfully in the characterization of the extreme elements of the class of 0,1-normalized convex games in Rosenmüller and Weidner [1974]. Although the fact that convex games and superadditive games have apparently similar definitions the representation approach for superadditive games has not been succesfull yet. For a detailed discussion of the used techniques and related subjects the reader is referred to Rosenmüller [1977].

\section{Concluding remarks}

The main open problem left here is the full characterization of the extreme elements of the class of non-negative superadditive games. The partial characterizations in the literature and the one presented in theorem 4.12 are not very promising in order to accomplish the full characterization. Eventually one expects that the approach of Rosenmüller and Weidner [1974] will be successful since it encloses a significant part of the techniques used in linear programming and convex analysis to characterize the extreme points of a polyhedral set. 


\section{Chapter 5}

\section{Upper bound sets for the class of superadditive games}

\subsection{Introduction}

In this chapter we will consider classes of games which fulfil some but not all of the superadditivity inequalities. In particular we will discuss games $v$ for which one of the following collection of inequalities hold.

$$
\begin{aligned}
& v(N) \geq v(S)+\sum_{j \in N \backslash S} v(\{j\}), \text { for each coalition } S, \\
& v(N) \geq v(S)+v(N \backslash S), \text { for each coalition } S, \\
& v(N) \geq \sum_{j \in J} v\left(S_{j}\right), \text { for each partition }\left(S_{j}\right)_{j \in J} \text { of } N .
\end{aligned}
$$

First observe that these conditions only put restrictions on the worth of the grand coalition in contrast with superadditivity. Furthermore, a game v for which (5.3) holds also fulfils (5.1) and (5.2). Recall that the inequalities (5.1) have been used in the characterization of games for which the core equals the domination core.

There are no established names for games for which one of the above sets of inequalities hold. Here we call a game $v$ with property (5.1) semistable; it is called splitting stable if (5.2) holds and partition atable if the game fulfils (5.3). A balanced game fulfils each of the three conditions since these conditions correspond to some of the balancedness constraints.

The contents

In the sections 5.2 and 5.3 we will provide procedures to represent a 
non-negative semistable game and a non-negative splitting stable game as a non-negative weighted sum of semistable splitting stable simple games respectively. In section 5.4 partition stable games are considered. It is shown that the class of non-negative partition stable games contains nonsimple extreme games. Furthermore a result is presented which states that superadditive covers of extreme partition stable games are extreme in the class of non-negative superadditive games. Unfortunately, the reverse does not hold as will be shown by an example.

\subsection{The semistable games}

Besides its role in the characterization of games with equal core and domination core (see subsection 1.2.2) there is another result in connection with the semistability property.

For an arbitrary game $v$ we call player $i$ a veto-player if $i$ is contained in each coalition $S$ for which we have

$$
v(S)>\sum_{j \in S} v(\{j\})
$$

This definition is clearly a generalization of a similar notion used in the context of simple games.

Theorem 5.1 Let $v$ be a game with veto-players. Then $v$ has a non-empty core if and only if it is semistable.

Proof:

('only if'-part) Non-emptiness of the core always implies that the game is semistable.

('if'-part) Let $i \in N$ be a veto-player of the semistable game $v$. Distribute the worth $v(N)$ among the players by allocating $v(\{j\})$ to all players $j \neq i$ and the remaing amount $v(N)-\sum_{j \in N \backslash\{i\}} v(\{j\})$ to player $i$. Denote this efficient allocation by $x$. Observe that $x$ is individual rational. If for a coalition $S$ we have $v(S) \leq \sum_{j \in S} v(\{j\})$ then also $v(S) \leq \sum_{j \in S} x_{j}$. Now suppose $v(S)>\sum_{j \in S} v(\{j\})$. Then $i \in S$, implying

$$
\sum_{j \in S} x_{j}=\sum_{j \in N} x_{j}-\sum_{j \in N \backslash S} x_{j}=v(N)-\sum_{j \in N \backslash S} v(\{j\}) \geq v(S)
$$

according to the semistability of $v$. This proves that $x$ is a core allocation of the game $v$. 
A semistable game without a veto-player may still have a non-empty core. To show this consider the game $v$ defined on page 140 where the worth of a coalition $S$ equals the maximum amount of pairs of players in $S$. We saw that this game is extreme in the class of non-negative superadditive games and has a non-empty core if and only if $|N|$ is even. Thus, $v$ is semistable and, furthermore, 0 -normalized which proves that there is no veto-player.

\section{Generating sets}

Both the class of semistable games, denoted by $\mathbf{S T}^{N}$, and the class $\mathbf{S T}_{+}^{N}$ of non-negative semistable games will be fully characterized here by giving a generating set, and characterizing the extreme directions respectively.

Theorem 5.2 The class $\mathbf{S T}^{N}$ of semistable games is the algebraic sum of the class of additive games and the cone generated by the 0 -normalized simple games and the games -1 , with $S \subset N,|S| \geq 2$.

Proof: For a semistable game $v$ its 0 -normalization $v_{0}=v-u$ is a semistable game, for which

$$
v_{0}(N) \geq v_{0}(S) \text { for each coalition } S, \text { and } v_{0}(N) \geq 0 .
$$

hold, with $u$ the additive game associated to $(v(\{i\}))_{i \in N}$. Let $v_{0}^{\prime}$ be the non-negative game, obtained from $v_{0}$ by changing negative worths into 0 , i.e.,

$$
v_{0}^{\prime}=v_{0}+\sum_{S \subset N_{,} v_{0}(S)<0}\left|v_{0}(S)\right|\left(-\mathbf{1}^{S}\right) .
$$

Then $v_{0}^{\prime}$ still fulfils (5.5). Now let $v_{0}^{\prime}$ be decomposed into a non-negative weighted sum of 0 -normalized $\{0,1\}$-valued games in the same way as is done for a non-negative monotonic game in the proof of theorem 1.22. One easily shows that these $\{0,1\}$-valued games fulfil (5.5), implying that they are simple. This proves that $v$ can be repregented as a non-negative weighted sum of an additive game and the games mentioned in the theorem. The proof is concluded with the observation that each of these games is semistable.

The mentioned games in the theorem form a generating set for $\mathbf{S T}_{0}^{N}$. However, not all of these games are extreme as can be observed from the fact that a 0-normalized simple game $w$ with $w(S)=0$ for a coalition $S \subset N$ can be decomposed into the sum of the game $-1 \mathbf{1}^{S}$ and 0 -normalized simple game $w+\mathbf{1}^{S}$.

For a semistable simple game $w$ it is possible that for one of the players, say $i \in N$, we have $w(\{i\})=1$. Of course, there can be only one player in $w$ 
with this property. Furthermore, for a coalition $S$ not containing $i$ we have $0 \leq w(S) \leq w(N)-w(\{i\})=1-1=0$, implying $w(S)=0$. Therefore, $w$ is either veto-controlled or 0 -normalized.

\section{The non-negative semistable games}

The simple temistable games are extreme in the cone of non-negative semistable games according to lemma 1.20, and there are no other extreme semistable games, up to a scalar multiplication factor. This is shown with the help of a 'slicing'-procedure to construct the weighted sum of simple semistable games for an arbitrary non-negative semistable game $v$.

\section{Procedure 5.3}

step 1 Let $k=0$ and $v^{0}=v$.

step 2 If $v^{k}(N)=\sum_{j \in N} v^{k}(\{j\})$ then start procedure 2.11 on the game $v^{k}$ and (core) allocation $(v(\{i\}))_{i \in N}$.

step 3 Let $w^{k}(S)=\left\{\begin{array}{ll}1 & \text { if } v^{k}(S)>0,|S| \geq 2, \\ 0 & \text { otherwise, }\end{array}\right.$ with $S \subseteq N$, and let $\alpha^{k}$ be the minimum of $v^{k}(N)-\sum_{j \in N} v^{k}(\{j\})$ and $\min \left(\left\{v^{k}(S)\right.\right.$ : $S \subseteq N$, with $\left.w^{k}(S)>0\right\}$ ).

Let $v^{k+1}=v^{k}-\alpha^{k} w^{k}$.

step 4 Increase $k$ by 1 ; GOTO step 2.

For a semistable game $v$ we have $v(N) \geq \sum_{j \in N} v(\{j\})$. If equality holds then each worth of a coalition $S$ is necessarily majorized by $\sum_{j \in S} v(\{j\})$, implying $(v(\{i\}))_{i \in N} \in$ Core $(v)$. In this case procedure 2.11 on page 47 is used in order to represent $v$ as a non-negative weighted sum of vetocontrolled simple games. Therefore, procedure 5.3 will stop whenever $v^{k}(N)$ equals $\sum_{j \in N} v^{k}(\{j\})$.

Suppose we are in Step 3 with a non-negative semistable game $v^{k}$. We, therefore, must have $v^{k}(N)>\sum_{j \in N} v^{k}(\{j\})$. The construction of the $\{0,1\}$ valued game $w^{k}$ and the weight $\alpha^{k}$ is such that $\alpha^{k}>0$ and $v^{k} \geq \alpha^{k} w^{k}$. Thus, the game $v^{k+1}$ is non-negative. Notice that $v^{k+1}(N)$ equals $v^{k}(N)-\alpha$ which majorizes $\sum_{j \in N} v^{k}(\{j\})=\sum_{j \in N} v^{k+1}(\{j\})$. Furthermore, for each coalition $S$ with $|S| \geq 2$ we have

- either $v^{k}(S)>0$; then

$$
v^{k+1}(N)-v^{k+1}(S)-\sum_{j \in N \backslash S} v^{k+1}(\{j\})
$$




$$
\begin{aligned}
& =v^{k}(N)-\alpha^{k}-v^{k}(S)+\alpha^{k}-\sum_{j \in N \backslash s} v^{k}(\{j\}) \\
& \geq 0
\end{aligned}
$$

- or $v^{k}(S)=0$; then

$$
\begin{aligned}
v^{k+1}(N)-v^{k+1}(S) & -\sum_{j \in N \backslash S} v^{k+1}(\{j\}) \\
& =v^{k}(N)-\alpha^{k}-\sum_{j \in N \backslash S} v^{k}(\{j\}) \\
& \geq v^{k}(N)-\sum_{j \in N} v^{k}(\{j\})-\alpha^{k} \\
& \geq 0 .
\end{aligned}
$$

We conclude that $v^{k+1}$ is semistable.

Assuming that $v=v^{0}$ is non-negative and semistable each of the constructed games $v^{k}$ is non-negative and semistable. Notice that the game $w^{k}$ is 0 -normalized and simple. If $\mathcal{N}(v)$ denotes the number of coalitions which have a worth not equal to 0 in a game $v$ then $\mathcal{N}\left(v^{k+1}\right) \leq \mathcal{N}\left(v^{k}\right)\left(\leq 2^{|N|}-1\right)$ since $0 \leq v^{k+1} \leq v^{k}$. The construction of $v^{k+1}$ is such that at least one coalition $S$ with $v^{k}(S)>0$ attains a zero worth in the game $v^{k+1}$, yielding $\mathcal{N}\left(v^{k+1}\right)<\mathcal{N}\left(v^{k}\right)$. The procedure, therefore, terminates after having constructed a finite total of 'slices', say $\alpha^{K} w^{K}$ is the last one (if no slices are constructed then we assume $K=-1$ ). Then

$$
v=v^{K+1}+\sum_{k=0}^{K} \alpha^{k} w^{k}
$$

and for the non-negative semistable game $v^{K+1}$ the equality $v^{K+1}(N)=$ $\sum_{j \in N} v^{K+1}(\{j\})$ holds. Using procedure 2.11 on $v^{K+1}$ with core allocation $\left(v^{K+1}(\{i\})\right)_{i \in N}$ we derive a representation of $v^{K+1}$ as a nonnegative weighted sum of veto-controlled simple games. We conclude that

Theorem 5.4 The class of non-negative semistable games is generated by its simple games. These games are either veto-controlled or O-normalized. 


\section{An example}

Let us illustrate procedure 5.3 with the help of the following 4-person semistable game $v$ which is neither superadditive nor has a core allocation. In the table the coalitions with zero worth are not shown.



Step 4: $k=1$.

Step 2: $v^{1}(N)=4>2=\sum_{j \in N} v^{1}(\{j\})$.

Step 3:

\begin{tabular}{l|llllll|l}
$w^{1}$ & 0 & 0 & 1 & 1 & 0 & 1 & $\alpha^{1}=2$ \\
$v^{2}$ & 1 & 1 & 1 & 0 & 0 & 2
\end{tabular}

Step 4: $k=2$.

Step 2: $v^{2}(N)=2=\sum_{j \in N} v^{2}(\{j\})$.

Start procedure 2.11 in Step 2, with $k=2$.

Step 2: $x^{2}=\left(v^{2}(\{i\})_{i \in N}=(1,1,0,0) \neq 0\right.$.

Step 3: Let $i$ be player 1 ;
\begin{tabular}{l|lll}
$w^{2}$ & 1 & 0 & 1
\end{tabular}
\begin{tabular}{l|ll}
$v^{3}$ & 0 & 1
\end{tabular}
0
0
0



Step 4: $k=3$.

Step 2: $x^{3} \neq 0$.

Step 3: Let $i$ be player 2;

\begin{tabular}{l|llllll|l}
$w^{3}$ & 0 & 1 & 0 & 0 & 0 & 1 & $\alpha^{3}=1$ \\
$v^{4}$ & 0 & 0 & 0 & 0 & 0 & 0 & $x^{4}=0$
\end{tabular}

Step 4: $k=4$.

Step 2: $x^{4}=0$; STOP.

\subsection{The splitting stable games}

We will present here a similar procedure in order to represent a nonnegative splitting stable game as a non-negative weighted sum of splitting stable simple games. 


\section{Procedure 5.5}

step 1 Let $k=0$ and $v^{0}=v$.

step 2 If $v^{k}(N)=0$ then STOP.

step 3 Choose an arbitrary player $i \in N$, and let $w^{k}$ be the game

$w^{k}(S)= \begin{cases}1 & \text { if } v^{k}(S)>0 \text { and } i \in S, \\ 1 & \text { if } v^{k}(S)>0, v^{k}(N \backslash S)=0, \text { and } i \in N \backslash S, \\ 0 & \text { otherwise, }\end{cases}$

with $S \subseteq N$, and let $\alpha^{k}=\min \left(\left\{v^{k}(S): S \subseteq N\right.\right.$ with $w^{k}(S)=$ 1\}).

Let $v^{k+1}=v^{k}-\alpha^{k} w^{k}$.

step 4 Increase $k$ by 1 ; GOTO step 2.

In case $v^{k}$ is non-negative then $v^{k+1}$ is non-negative, and the latter game must have at least one more coalition with zero worth than $v^{*}$. This proves that the procedure eventually terminates, provided that it is started with a non-negative game $v$. One immediately observes that in this case the $\{0,1\}$-valued games $w^{k}$ are simple since $v^{k}(N)>0$, for each $k$. Furthermore, these games are splitting stable by construction. Now let us suppose that $v^{k}$ is splitting stable. Let $i \in N$ be the player chosen in step 3. For each splitting up of the grand coalition $N$ into two coalitions $S$ and $N \backslash S$ the following three cases occur.

- Player $i$ is a member of one of the coalitions, say $S$, with positive worth. Then $w^{k}(S)=1$ and $w^{k}(N \backslash S)=0$, implying

$$
\begin{aligned}
v^{k+1}(N)-v^{k+1}(S) & -v^{k+1}(N \backslash S) \\
& =v^{k}(N)-\alpha^{k}-\left(v^{k}(S)-\alpha^{k}\right)-v^{k}(N \backslash S) \\
& \geq 0
\end{aligned}
$$

- The coalition containing player $i$, say $i \in N \backslash S$, has worth 0 , and the other has positive worth. Then $w^{k}(S)=1$ and $w^{k}(N \backslash S)=0$, implying

$$
\begin{aligned}
v^{k+1}(N)-v^{k+1}(S) & -v^{k+1}(N \backslash S) \\
& =v^{k}(N)-\alpha^{k}-\left(v^{k}(S)-\alpha^{k}\right)-v^{k}(N \backslash S) \\
& \geq 0
\end{aligned}
$$




$$
\begin{aligned}
& -v^{k}(S)=0 \text { and } v^{k}(N \backslash S)=0 \text {. Then } w^{k}(S)=0 \text { and } w^{k}(N \backslash S)=0, \\
& \text { implying } \\
& \quad v^{k+1}(N)-v^{k+1}(S)-v^{k+1}(N \backslash S)=v^{k}(N)-\alpha^{k} \geq 0
\end{aligned}
$$

We conclude that $v^{k+1}$ is splitting stable. Thus, the splitting stability of $v=v^{0}$ implies the splitting stability of each of the computed games $v^{k}$ and in particular of $v^{K+1}$, where the number $K$ is such that $\alpha_{K} w^{K}$ is the last slice computed, with $K \geq-1$. Then

$$
v=v^{K+1}+\sum_{k=0}^{K} \alpha^{k} w^{k}
$$

The splitting stability property of $v^{K+1}$ and the fact that the worth of the grand coalition is 0 implies that this game clearly equals the all-losing game.

We conclude that the procedure provides the required representation for $v$. Thus,

Theorem 5.6 The class of non-negative splitting stable games is generated by its simple games which are the extreme directions, up to a positive multiplication factor.

\subsection{The partition stable games}

For a game $v$ and its superadditive minimum cover $v_{\min }$ the worths $v(N)$ and $v_{\min }(N)$ are equal if and only if $v$ is partition stable (see also the following lemma). For partition stable games one might expect that after the grand coalition is formed there will be no splitting off into smaller coalitions.

The existence of non-simple extreme partition stable games

Of course, the non-negative partition stable games form a pointed polyhedral cone. Therefore, the extreme directions form a generating set. As in the case of non-negative superadditive games there are non-simple extreme partition stable games. To show this consider the five-person game $v$ in which all two-person coalition have worths 1 and the grand coalition has worth 2. The other coalitions are assumed to 
have worths 0 . Now suppose there is a partition stable simple game $w$ and $\alpha>0$ such that the game $v-\alpha w$ is partition stable. We must have $w(S)+w(T)=1=w(N)$ for all two-person disjoint coalitions $S, T \subset N$. Without loss of generality assume that $w(\{1,2\})=1$. This implies $w(\{3,4\})=w(\{3,5\})=0$. From $w(\{3,4\})=0$ we obtain $w(\{1,5\})=1$ and, similarly, $w(\{3,5\})=0$ implies $w(\{2,4\})=1$. Then $2=w(\{1,5\})+w(\{2,4\})=w(N)=1$; a contradiction. We conclude that $v$ cannot be represented as a positive weighted sum of partition stable simple games.

\section{The stable coalitions}

The extreme partition stable games can be of help in providing extreme superadditive games, as the following theorem shows. We will first prove a preliminary result.

In a game $v$ we will call a coalition $S$ stable if $v(S) \geq \sum_{k \in K} v\left(S_{k}\right)$ for each partition $\left(S_{k}\right)_{k \in K}$ of $S$. Thus, a game is partition stable whenever the grand coalition is stable.

Lemma 5.7 For a game $v$ and its superadditive cover $v_{\min }$ we have $v_{\min }(S)=v(S)$ if and only if coalition $S$ is stable in $v$.

Proof: Consider the game $\bar{v}$ defined by

$$
\bar{v}(S)=\max \left(\left\{\sum_{k \in K} v\left(S_{k}\right):\left(S_{k}\right)_{k \in K} \text { partition of } S\right\}\right), \quad S \subseteq N
$$

This game is superadditive and majorizes $v$. Therefore it also majorizes the superadditive cover $v_{\min }$. For stable coalitions $S$ in $v$ we have equality $v(S)=\bar{v}(S)$ and, therefore also $v(S)=v_{\min }(S)$.

For a coalition $S$ which is not stable, say $v(S)<\sum_{k \in K} v\left(S_{k}\right)$ for a partition $\left(S_{k}\right)_{k \in K}$, we have

$$
\sum_{k \in K} v\left(S_{k}\right) \leq \sum_{k \in K} v_{\min }\left(S_{k}\right) \leq v_{\min }(S)
$$

thus strict inequality $v(S)<v_{\min }(S)$ holds.

In general the equality $v_{\min }=\bar{v}$ holds as one can easily obtain from the above observations. 
Theorem 5.8 Let $v \neq 0$ be an extreme game in the class of nonnegative partition stable games. Then the superadditive cover $v_{\min }$ of $v$ is extreme in the closs of non-negative superadditive games.

Proof: Let $S$ not be a stable coalition of $v$, i.e., there is a partition $\left(S_{k}\right)_{k \in K}$ of $S$ such that $v(S)<\sum_{k \in K} v\left(S_{k}\right)$. Of course, $S$ is not equal to $N$. Suppose $v(S)>0$. Then an $\epsilon>0$ exists such that the games $v+\epsilon \mathbb{1}^{S}$ and $v-\epsilon \mathbf{1}^{S}$ are partition stable and non-negative. The sum of these two games equals $2 v$ and since $v$ is extreme we conclude that $v+\epsilon \mathbb{1}^{S}$ and $v-\epsilon \mathbb{1}^{S}$ are positive multiples of $v$, implying that $v$ itself is a multiple of the game $1^{S}$. Now the game $\mathbb{1}^{S}$ is partition stable if and only if $S=N$. We conclude that the assumption $v(S)>0$ cannot hold, i.e., $v(S)=0$.

Now suppose $v_{\min }=w^{1}+w^{2}$ with $w^{1}$ and $w^{2}$ non-negative superadditive games. Let $v^{1}$ and $v^{2}$ be games which equal $w^{1}$ and $w^{2}$ respectively, for all coalitions $S$ which are stable in $v$ and 0 otherwise. Thus, $v^{1}$ and $v^{2}$ are partition stable games. First we will prove that $v$ equals the sum of $v^{1}$ and $v^{2}$.

If $S$ is non-stable in $v$ then $v(S)=0=v^{1}(S)+v^{2}(S)$ and if $S$ is stable in $v$ then $v(S)=v_{\min }(S)$ according to lemma 5.7, implying $v(S)=w^{1}(S)+w^{2}(S)=v^{1}(S)+v^{2}(S)$. We conclude that $v=v^{1}+v^{2}$. Again using the extremality of $v$, both $v^{1}$ and $v^{2}$ are non-negative multiples of $v$. Then also $v_{\min }^{1}$ and $v_{\min }^{2}$ equal $v_{\min }$ up to a positive multiplication factor. Moreover, $v_{\min }^{1}+v_{\min }^{2}=v_{\min }=w^{1}+w^{2}$. Since $v^{1} \leq w^{1}$ and $v^{2} \leq w^{2}$ we have $v_{\min }^{1} \leq w^{1}$ and $v_{\min }^{2} \leq w^{2}$ according to lemma 4.5. We conclude that $v_{\min }^{1}=w^{1}$ and $v_{\min }^{2}=w^{2}$, yielding the extremality of $v_{\min }$ in the class $\mathbf{S A}_{+}^{N}$ of non-negative superadditive games.

As for the class of non-negative superadditive games there is no full characterization available of the extreme games in the cone of nonnegative partition stable games.

\section{An example}

An example of an extreme superadditive game which is not extreme in the class of non-negative partition stable games is the following 4person game $v$ with $v(S)=1$ for coalitions $S$ with 2 or 3 players and $v(N)=2$. The non-mentioned coalitions have worths 0 . This 
superadditive game is extreme according to theorem 4.12 , and is the sum of the superadditive simple game $w^{11}$ with $w^{1}(S)=\mathbb{1}$ for all $S$ with at least 3 players and the sets $\{1,2\},\{1,3\}$ and $\{1,4\}$, and the partition stable simple game $w^{2}$ which assigns 1 only to coalitions $\{2,3\},\{2,4\}$, $\{3,4\}$ and $N$ (notice that the games $v$ and $w^{1}$ have a non-empty core). Therefore, $v$ is not extreme as a partition stable game.

We will show that $v$ cannot be the superadditive cover of an extreme game in the class of non-negative partition stable games. If $w$ is a partition stable game with $w_{\min }=v$ then we must have $w(S)=v(S)=$ 1 for each two-person coalition $S$. Furthermore, according to lemma 5.7 we have $w(N)=w_{\min }(N)=v(N)=2$. Observe that if the other coalitions have worths zero then $w_{\min }=v$ holds. The extremality of $w$ as a partition stable game now implies that the other coalitions indeed have worths zero.

Consider the two simple games $w^{2}$ as defined before, and $u$ defined by $u(S)=1$ only for $S=\{1,2\},\{1,3\}$ and $\{1,4\}$, and $N$. Then $w=u+w^{2}$ and $w$ is not a positive multiple of one of these games. The partition stability of both games $u$ and $w^{2}$ contradicts the extremality assumption of $w$. 



\section{Bibliography}

Assad, A.A. [1978] Multicommodity network flows-a survey. Networks, 8:37-91.

Aumann, R.J. and M. Maschler [1964] The bargaining set for cooperative games. In M. Dresher, L.S. Shapley, and A.W. Tucker, editors, Advances in Game Theory, pages 443-476, Princeton University Press, Princeton, New Jersey.

Bondareva, O.N. [1962] The core of an n-person game (in Russian). Vestnik Leningrad University, 17, No. 13:141-142. English translation: Selected Russian papers on game theory, 1959-65, Economic Research Program, Princeton University, Princeton, pages 29-31, April 1968.

Bondareva, O.N. [1963] Some applications of linear programming methods to the theory of cooperative games (in Russian). Problemy Kibernetiki, 10:119-139. English translation. U.S. Dept. of Commerce, Office of Technical Services, Washington, D.C., Sept. 1964.

Charnes, A. and K.O. Kortanek [1967] On balanced sets, cores, and linear programming. Cahiers du Centre dËtudes de Recherche Opérationelle, 1:32-43.

Curiel, I.J. [1988] Cooperative Game Theory and Applications. Ph.D. Thesis, Nijmegen, The Netherlands.

Curiel, I.J., J.J.M. Derks and S.H. Tijs [1989] On balanced games and flow games with committee control. OR Spektrum, 11:83-88.

Dantzig, G.B. and D.R. Fulkerson [1956] On the max-flow min-cut theorem of networks. In H.W. Kuhn and A.W. Tucker, editors, Linear Inequalities and Related Systems, pages 215-221, Princeton University Press, Princeton, New Jersey.

Davis, M. and M. Maschler [1965] The kernel of a cooperative game. Naval Research Logistics Quarterly, 12:223-259.

Derks, J.J.M. [1987a] Decomposition of games with non-empty core into veto-controlled simple games. OR Spektrum, 9:81-85. 
Derks, J.J.M. [1987b] On the extreme elements of the class of $(0,1)$ normalized superadditive games. In H.J.M. Peters and O.J. Vrieze, editors, Surveys in Game Theory and Related Topics, CWI Tract 39, Centre for Mathematics and Computer Science, Amsterdam, 215-228.

Derks, J.J.M. [1989] On systems of linear inequalities without strongly redundant constraints. Report M89-12, Department of Mathematics, University of Limburg, Maastricht, The Netherlands.

Derks, J.J.M. and T.S.H. Driessen [1990] A decomposition of the class of semiconvex games. Report M90-08, Department of Mathematics, University of Limburg, Maastricht, The Netherlands.

Derks, J.J.M. and J. Kuipers [1991] Cover games. Report M91-05, Department of Mathematics, University of Limburg, Maastricht, The Netherlands.

Derks, J.J.M. and S.H. Tijs [1985] Stable outcomes for multi-commodity flow games. Methods of Operations Research, 50:493-504.

Derks, J.J.M. and S.H. Tijs [1986] Totally balanced multi-commo-dity games and flow games. Methods of Operations Research, 54:335-347.

Dragan, I., J. Potters and S. Tijs [1989] Superadditivity for solutions of coalitional games. Libertas Mathematica, 9:101-110.

Driessen, T.S.H. [1985] Properties of 1-convex n-person games. OR Spektrum, 7:19-26.

Driessen, T.S.H. [1988] Cooperative Games, Solutions and Applications. Kluwer Academic Publisher, Dordrecht, The Netherlands.

Driessen, T.S.H. and S.H. Tijs [1983] The $\tau$-value, the nucleolus and the core for a subclass of games. Methods of Operations Research, 46:395406.

Driessen, T.S.H. and S.H. Tijs [1985] The $\tau$-value, the core and semiconvex games. International Journal of Game Theory, 14:229-247.

Dubey, P. $[\mathbf{1 9 7 5}]$ On the uniqueness of the Shapley value. International Journal of Game Theory, 4:131-139.

Dubey, P. and L.S. Shapley [1984] Totally balanced games arising from controlled programming problems. Mathematical Programming, 29:245-267. Ford, L.R. and D.R. Fulkerson [1956] Maximal flow through a network. Canadian Journal of Mathematics, 8:399-404.

Gillies, D.B. [1953] Some Theorems on n-Person Games. Ph.D. Thesis. Princeton University Press, Princeton, New Jersey.

Granot, D. [1986] A generalized linear production model: a unifying model. Mathematical Programming, 34:212-222. 
Griesmer, J.H. [1959] Extreme games with three values. Contributions to the theory of games IV, Annals of Mathematics Studies, 40:189-212.

Gurk, H.M. [1959] Five person, constant sum, extreme games. Contributions to the theory of games IV, Annals of Mathematics Studies, 40:179-188. Harsanyi, J.C. [1963] A simplified bargaining model for the n-person cooperative game. International Economic Review, 4:194-220.

Ichishi, T. [1981 \ Super-modularity: applications to convex games and to the greedy algorithm for LP. Journal of Economic Theory, 25:283-286.

Kalai, E. and E. Zemel [1982a] Generalized network problems yielding totally balanced games. Operations Research, 30:998-1008.

Kalai, E. and E. Zemel [1982b] Totally balanced games and games of flow. Mathematics of Operations Research, 7:476-478.

Karwan, M.H., M.H. Lofti, J. Telgen and S. Zionts [1983] editors, Redundancy in Mathematical Programming: A State of the Art Survey. Springer-Verlag, Berlin.

Kennington, J. [1978] A survey of linear cost multicommodity network flows. Operations Research, 6:209-236.

Klee, V. [1959] Some characterizations of convex polyhedra. Acta Mathematica, 102:79-107.

Littlechild, S.C. [1974] A simple expression for the nucleolus in a special case. International Journal of Game Theory, 3:21-29.

Littlechild, S.C. and G. Owen [1973] A simple expression for the shapley value in a special case. Management Science, 20:370-372.

Luce, R.D. and H. Raiffa [1957] Games and Decisions. John Wiley and Sons, New York.

Maschler, M., B. Peleg and L.S. Shapley [1972] The kernel and bargaining set for convex games. International Journal of Game Theory, 1:7393.

Milnor, J.W. [1952] Reasonable outcomes for n-person games. Technical Report, RM 916, The RAND Corporation, Santa Monica, California.

Moulin, H. [1989] Cores and large cores when population varies. Mimeo, Department of Economics, Duke University.

Muto, S., J. Potters and S. Tijs [1989] Information market games. International Journal of Game Theory, 18:209-226.

O'Neill, B. [1982] A problem of rights arbitration from the Talmud. Mathematical Social Sciences, 2:345-371.

Neumann, J. von, and O. Morgenstern [1944] Theory of Games and Economic Behavior. Princeton University Press, Princeton, New Jersey. 
Nouweland, A. van den, H. Aarts and P. Borm [1989] Multi-commodity games. Methods of Operations Research, 63:329-338.

Nouweland, A. van den, J. Potters, S. Tijs and J. Zarzuelo [1990] Cores and related Solution Concepts for Multi-Choice Games. FEW 478, Department of Economics, Tilburg University, The Netherlands.

Owen, G. [1975] On the core of linear production games. Mathematical Programming, 9:358-370.

Owen, G. [1982] Game Theory. (Second edition). Academic Press, New York.

Peleg, B. [1986] On the reduced game property and its converse. International Journal of Game Theory, 15:187-200.

Potters, J.A.M. [1987] Linear optimization games. In H.J.M. Peters and O.J. Vrieze, editors, Surveys in Game Theory and Related Topics, CWI Tract 39, Centre for Mathematics and Computer Science, Amsterdam, 251-276.

Potters, J., R. Poos, S. Tijs and S. Muto [1989] Clan games. Games and Economic Behavior, 1:275-293.

Rockafellar, R.T. [1970] Convex Analysis. Princeton University Press, Princeton, New Jersey.

Rosenmüller, J. [1977] Extreme Games and their Solutions. Volume 145 of Lecture notes in Economics and Mathematical Systems, Springer-Verlag, Berlin.

Rosenmüller, J. [1981] The Theory of Games and Markets. North-Holland Publishing Company, Amsterdam.

Rosenmüller, J. and H.G. Weidner [1974] Extreme convex set functions with finite carrier: general theory. Discrete Mathematics, 10:343-382. Schmeidler, D. [1969] The nucleolus of a characteristic function game. SIAM Journal of Applied Mathematics, 17:1163-1170.

Shapley, L.S. [1953] A value for n-person games. In H.W. Kuhn and A.W. Tucker, editors, Linear Inequalities and Related Systems, pages 307318, Princeton University Press, Princeton, New Jersey.

Shapley, L.S. [1956] Simple games. an outline of the descriptive theory. Behavioral Science, 7:59-66.

Shapley, L.S. [1959] Equilibrium points in games with vector payoffs. Naval Research Logistics Quarterly, 6:57-61, 1959.

Shapley, L.S. [1967] On balanced sets and cores. Naval Research Logistics Quarterly, 14:453-460.

Shapley, L.S. [1971] Cores of convex games. International Journal of Game Theory, 1:11-26. 
Shubik, M. [1971] The 'Bridge Game economy: an example of indivisibilities. Journal of Political Economy, 79:909-912.

Shubik, M. [1982] Game Theory in the Social Sciences. Cambridge MIT Press.

Sobolev, A.I. [1975] The characterization of optimality principles in cooperative games by functional equations (in Russian, English summary). In N.N. Vorobjev, editor, Matematischeskie Metody v Socialnix Naukax (Issue 6), pages 94-151, Academy of Sciences of the Lithuanian SSR, Vilnius.

Spinetto, R.D. [1971] Solution Concepts of n-Person Cooperative Games as Points in the Game Space. Ph.D. Thesis, technical report, No. 138, Department of Operations Research, Cornell University Ithaca, New York. Spinetto, R.D. [1974] The geometry of solution concepts for n-person cooperative games. Management Science C, 20:1292-1299.

Sprumont, Y. [1990] Population monotonic allocation schemes for cooperative games with transferable utility. Games and Economic Behavior, 2:378-394.

Telgen, J. [1979] Redundancy and Linear Programs. Ph. D. Thesis, Mathematisch Centrum, Amsterdam.

Tijs, S.H. [1981] Bounds for the core and the $\tau$-value. In O. Moeschlin and D. Pallaschke, editors, Game Theory and Mathematical Economics, pages 123-132, North-Holland Publishing Company, Amsterdam.

Tijs, S.H. and F.A.S. Lipperts [1982] The hypercube and the core cover of $n$-person cooperative games. Cahiers du Centre dÉtudes de Recherche Opérationelle, 24:27-37.

Weber, R.J. [1988] Probabilistic values for games. In A.E. Roth, editor, The Shapley Value, pages 101-119, Cambridge University Press, Cambridge. Zeleny, M. [1975] Games with multiple payoffs. International Journal of Game Theory, 4:179-191. 



\section{Author index}

Aarts, H. 65,66

Assad, A.A. 74

Aumann, R.J. 16

Bondareva, O.N. 7, 40

Borm, P.E.M. 65, 66

Charnes, A. 40

Curiel, I.J. 43, 57, 76

Dantzig, G.B. 74

Davis, M. 16

Derks, J.J.M. 43, 45, 49, 57, 63, $65,74,76,77,99,125,131$, 137

Dragan, I. 17, 35

Driessen, T.S.H. 12, 17, 19, 86, $92,97,99,121,122$

Dubey, P. 57, 59, 116

Ford, L.R. 74

Fulkerson, D.R. 74

Gillies, D.B. 7

Granot, D. 57

Griesmer, J.H. 142

Gurk, H.M. 141, 143

Harsanyi, J.C. 119

Ichiishi, T. 11

Kalai, E. $59,68,74,75$
Karwan, M.H. 63

Kennington, J. 74

Klee, V. 21, 24, 25

Kortanek, K.O. 40

Kuipers, J. 131

Lipperts, F.A.S. 11, 12, 13, 14

Littlechild, S.C. 18, 54

Lofti, M.H. 63

Luce, R.D. ix, 13

Maschler, M. 16

Milnor, J.W. 13

Morgenstern, $O$. ix, 141

Moulin, H. 50

Muto, S. 95,117

Neumann, J. von ix, 141

Nouweland, A. van den 9,65, 66

O'Neill, B. 103

Owen, G. ix, 18, 54, 56, 64

Peleg, B. 16,35

Poos, R. 95

Potters, J.A.M. 9, 17, 35, 58, 59, 95,117

Raiffa, H. ix, 13

Rockafellar, R.T. 10, 25

Rosenmüller, J. ix, xi, 102, 143

Schmeidler, D. 16 
Shapley, L.S. $3,7,11,16,40,41$,

$57,59,64,116,117,119$,

$130,132,134$

Shubik, M. ix, 140

Sobolev, A.I. 16

Spinetto, R.D. 38, 41, 140

Sprumont, Y. 50, 51

Telgen, J. 63

Tijs, S.H. 9, 11, 12, 13, 14, 15, 17, $35,43,57,65,74,76,77$, $92,97,117,122$

Weber, R.J. 10

Weidner, H.G. 102, 143

Zarzuelo, J. 9

Zeleny, M. 64

Zemel, E. $59,68,74,75$

Zionts, S. 63 


\section{Subject index}

0,1-normalization $3,38,41,141$

0-normalization $3,38,86,92,100$, 104,114

action set 58

airport cost function 18,121

allocation 5

concession 122

core $6,36,47$

dominated 8

efficient 5, 49

individually rational 6

marginal contribution 11, 39, $84,93,97,103$

marginal worth 9

maximal remainder $13,98,103$

arc set 70

balancedness 5

collection of vectors 57

collection of weights 5,130

minimum cover 131

multicommodity game 65

set of coalitions 5

vector 25,41

base 116

Bondareva-Shapley correspondence

$$
40,44,140
$$

bottom element 22

capacitated flow model 70

capacity 70 cut 72

flow model 71 capacity function 70 characteristic function 2 coalition 2

clan 95

essential 130

losing 3

minimal winning 130

stable 153

winning 3,41

commodity space 64,70

comprehensive set 64,70

cone 20,132

minimum closed 126

pointed 24

polyhedral 21

proper 129

subgame closed 133

conservation conditions 71

constraint vector $\mathbf{5 5}$

sharp 63,65

strongly redundant 63

control 57

cornmittee 57

dictatorial 74

control function $\mathbf{7 4}$

controlled linear programming model 56

core $6,35,63$

allocation 6,66 
catcher 9

multicommodity 66

superadditivity 35

core cover $14,103,109$

cut 71

decomposable game 133

decomposable set of coalitions 134

derivative 119

divident 119

domination core 8,146

Duality Theorem $40,55,60,74$

evaluating function 58

extremality correspondence $x i$

extreme direction 24

extreme element 22

extreme game 29

feasible region 55

flow 71

feasible 71

value 71

flow capacity 71

game 2

1-convex $12,90,102,113,121$

additive $6,26,29,69,113,128$

airport cost savings $19,52,98$, $104,110,120$

all-losing 3

all-winning 3

balanced 5

bankruptcy 103

cardinality 2

clan 95

constant sum 141

constraint $\mathbf{5 7}$

convex 4, 11

cooperative 2 cost 19,121

dictator 4

dual 19

flow 71

one-commodity 73

glove 2, 139

ideally covered 15,110

linear production 56

generalized 57

multicommodity 65

veto-controlled 57

linear programming 56

maximally blocked $12,90,113$,

121

monotonic $4,50,128,133$

non-negative 3

optimization 59

partition stable 145,152

quasi-balanced $15,103,113,121$

semibalanced $13,84,97,113$, 120,121

semiconvex $97,113,119,121$

semistable 145

simple 3, 29

veto-controlled $3,41,57,149$

splitting stable 145,150

superadditive $4,137,145,152$

three-valued 142

totally balanced $5,51,130,140$

unanimity 4, 119

uniform 137

unity 4,113

gap function 20,121

generating set 21

imputation set 6,35

K-chain 142

linear mapping $26,42,87,93$ 
$\Omega$-injective $28,35,89,94$

marginal core $11,90,109$ marginal gap 11, 37, 84, 91 Max-Flow-Min-Cut theorem 74 maximal remainder 13

Milnor set 13, 97 minimum cover 131

balanced 131 monotonic 131 superadditive 131,153 totally balanced 131 mixture 22, 28, 42, 45 monotonicity 4 set of coalitions 51 simple game 51 vector 25

multicommodity allocation 66 multicommodity core 66 multicommodity game 64 additive 76 balanced 65

flow 81 polyhedral 81 quasi-additive $68,75,81$ strictly balanced 77 totally balanced 81

node set 70

optimization model 59

balanced 59

linear 59

\section{Pareto-optimality $\mathbf{7 7}$}

player 1

fictitious 143

veto $3,41,146$

population monotonic allocation scheme 50 price vector 56 stable 61

proper set of coalitions 132

representative set 24

savings game 19,121

savings function 19

Separation Theorem 10, 22, 40

Shapley value 16,54

sink 70

solution scheme 6,16

source 70

strategical equivalence 3,29

strict convexity 78

subbalancedness $\mathbf{5 1}$

subgame 2, 60, 69, 133

superadditivity 4

core 35

vector 128

T-value $17,97,109$

top element 22

total balancedness 5 collection of vectors 57,130 minimum cover 131 vector 25

veto-control 3

flow model 76 set of coalitions 51 simple game 3,42

voting situation 3

Weber set 10, 16, 99

worth 2 



\section{Samenvatting}

\section{Over polyhedrale kegels van coöperatieve spelen}

Het spelmodel dat centraal staat in dit proefschrift is afkomstig uit de Coöperatieve Speltheorie en bestaat uit een tweetal componenten: een eindige verzameling van spelers en een reëelwaardige funktie, de zogenaamde karakteristieke funktie. Het staat de spelers vrij onderling coalities aan te gaan; de karakteristieke funktie geeft weer welke besparingen of winsten gekoppeld zijn aan zulke coalities.

In dit model zijn twee probleemstellingen te onderscheiden: welke coalities zullen worden gevormd en hoe groot is de (relatieve) sterkte van de betrokken spelers. In dit proefschrift zullen we verscheidene eigenschappen voor spelen behandelen die de formatie van de grote coalitie, de coalitie van alle spelers, aanmoedigt, dan wel waarschijnlijk maakt. In deze context kan de tweede probleemstelling vertaald worden naar hoe de besparingen, gekoppeld aan de grote coalitie, moeten worden verdeeld onder de spelers.

Een verdelingsmethode die voor een spel aangeeft, hoe de genoemde besparingen te verdelen, wordt een oplossingsconcept genoemd. $\mathbf{E r}$ zijn tal van oplossingsconcepten beschikbaar; elk van deze concepten is gebaseerd op verschillende inzichten met betrekking tot het beschrijven of definiëren van de sterkte van een speler in een spel. Verder heeft ieder concept zijn eigen klasse van spelen waarvoor de methode stabiele of redelijke verdelingen voorstelt.

Het meest toegepaste (meerwaardige) oplossingsconcept is wellicht de 
core. Deze bestaat uit die verdelingen van de besparingen (bij de vorming van de grote coalitie) onder de spelers waarbij geen enkele coalitie er beter wan wordt als deze zich alsnog afsplitst van de grote coalitie. Zulke core-verdelingen hoeven voor een spel echter niet te bestaan of zijn slechts met lineaire programmeringstechnieken te berekenen. Men heeft dan ook vele typen van spelen bestudeerd die een gemakkelijk te bepalen core bezitten of waarvoor er een niet-lege alternatieve verzameling van verdelingen bestaat welke de core omvat.

In dit proefschrift beschouwen we verschillende van zulke typen van spelen. Onze eerste doelstelling is inzicht te verwerven in de struktuur van de corresponderende klassen van spelen. De restricties van deze klassen met betrekking tot de 0-genormaliseerde spelen en de nietnegatieve spelen worden eveneens in beschouwing genomen. Vooral de beperking tot de niet-negatieve spelen is een standaard restrictie in het geval negatieve besparingen of winsten betekeningsloos zijn.

De ruimte van spelen wordt beschouwd als een Euclidische vectorruimte, met dimensie gelijk aan het totale aantal niet-lege coalities. De meeste van de door ons beschouwde klassen van spelen zijn polyhedrale kegels, d.w.z. ze kunnen worden beschreven als een eindige doorsnede van gesloten halfruimten. Volgens een bekend dualiteitsprincipe wordt een polyhedrale kegel vastgelegd door een eindige genererende deelverzameling, d.w.z. een eindige deelverzameling van elementen met de eigenschap dat ieder element in de kegel kan worden beschreven als een niet-negatieve gewogen som van elementen uit de deelverzameling. Specifieke randelementen van de kegel, de zogenaamde extreme richtingen, zijn bevat in elke genererende verzameling (op een vermenigvuldigingsfactor na).

Het bepalen van (de) extreme richtingen van een klasse van spelen en de constructie van een genererende verzameling hebben niet alleen een wiskundige maar ook een speltheoretische waarde. In de eerste plaats is het relevant het gedrag van een oplossingsconcept op een genererende verzameling te kennen om zodoende het gedrag te kunnen beschrijven met betrekking tot de kegel die voortgebracht wordt door die genererende verzameling. In aansluiting hierop kan men stellen dat als een oplossingsconcept gebaseerd is op onbetrouwbare rationaliteitsprincipes, dit dan duidelijk zou moeten worden door de verdelingen die dit concept toekent aan de extreme spelen. Rosenmüller [1977] drukt zich zelfs sterker uit: Als een oplossingsconcept gebaseerd is op een spe- 
cifiek sociaal gedrag en als dit concept beschouwd wordt op de klasse van spelen waarvoor het concept aanvaardbare verdelingen toekent dan zou extremiteit van een spel in geometrische zin moeten overeenkomen met een notie van extremiteit in de zin van sociaal gedrag. We zullen deze overeenkomst in extremiteit inderdaad enkele keren aantreffen.

De inhoud van het proefschrift is als volgt. Allereerst worden de noodzakelijke speltheoretische begrippen ingevoerd en verduidelijkt. We gaan in op eigenschappen van spelen zoals superadditiviteit, convexiteit, gebalanceerdheid, semigebalanceerdheid en quasigebalanceerdheid. Verder behandelen we een aantal meerwaardige oplossingsconcepten zoals de core, de dominantie core, de marginale core en de core cover. De laatste twee concepten kunnen worden beschouwd als relaxaties van het core concept. Opgenomen zijn ook twee enkelwaardige oplossingsconcepten: de Shapley waarde en de $\tau$-waarde. Ook zullen we dieper ingaan op enkele begrippen uit de convexe analyse en worden enkele elementaire resultaten gegeven.

In hoofdstuk 2 behandelen we klassen van spelen met een niet-lege core. Ons hoofdresultaat is de karakterisering van de extreme spelen van de klasse van niet-negatieve spelen met een niet-lege core. Deze extreme spelen komen overeen met de $\{0,1\}$-waardige spelen waarvoor er zogenaamde veto-spelers bestaan. Deze spelen voldoen dan ook aan een notie van extreem sociaal gedrag zodat de core een voorbeeld van een oplossingsconcept is waarop de extremiteitsovereenkomst van toepassing is. Ons hoofdresultaat wordt verder toegepast in een alternatief bewijs van de welbekende Bondareva-Shapley correspondentie, die stelt dat de gebalanceerdheid van een spel equivalent is met het niet-leeg zijn van de core. Ook zullen we nog een aantal deelklassen van spelen met een niet-lege core karakteriseren door middel van de constructie van genererende verzamelingen. Hoofdstuk 2 wordt beëindigd met een overzicht van toepassingen van het hoofdresultaat in situaties waarin $\{0,1\}$-waardige spelen gebruikt worden ter modellering van comitécontrole. Het overzicht behandelt de lineaire programmeringsspelen, goederenspelen en (goederen)stroomspelen.

Hoofdstuk 3 beschrijft de karakteriseringen van een aantal klassen van spelen met de semigebalanceerdheidseigenschap. Naast de semigebalanceerde spelen behandelen we de 1-convexe spelen (spelen waarvoor de niet-lege core gelijk is aan de marginale core) en verwante spelen zoals de maximaal geblokte spelen (spelen met een niet-lege marginale 
core) en de clan spelen. Verder komen ook de semiconvexe spelen en de quasigebalanceerde spelen aan de orde. Aan het einde van dit hoofdstuk geven we een overzicht van de resultaten.

Hoofdstuk 4 betreft een onderzoek van kegels van spelen welke gesloten zijn onder de minimum operatie. De klasse van niet-negatieve superadditieve spelen is een welbekend voorbeeld. We zullen de gevonden resultaten dan ook op deze klasse toepassen, resulterend in een gedeeltelijke karakterisering van de extreme spelen.

Een andere benadering van de karakterisering van deze extreme spelen (een algehele karakterisering is nog onbekend) is het afzwakken van de superadditiviteitseigenschap. Het laatste hoofdstuk is gewijd aan drie klassen van spelen die op deze wijze ontstaan. Een resultaat met betrekking tot een van deze klassen laat zien dat haar extreme spelen inderdaad gebruikt kunnen worden ter bepaling van extreme superadditieve spelen. Een andere klasse is verwant met spelen waarvoor de core overeen komt met de dominantie core. 


\section{Curriculum vitae}

De auteur werd geboren op 9 april 1956 te Roermond. Na het behalen van het eindexamen gymnasium $\beta$ in 1975 studeerde hij wiskunde aan de Katholieke Universiteit te Nijmegen. In 1979 rondde hij het kandidaatsexamen af met bijvakken sterrenkunde en informatica. Het doctoraalexamen werd behaald in 1983 met als hoofdrichting besliskunde en bijvakken stochastiek en economie. Hij schreef zijn doctoraalscriptie onder begeleiding van Prof.Dr. S.H. Tijs. Vanaf 1984 was hij gedurende twee en een half jaar werkzaam als wetenschappelijk medewerker bij de sectie wiskunde in Nijmegen, onder supervisie van Prof. Tijs. Een van de onderzoeksonderwerpen richtte zich op een speltheoretische benadering van een goederenstroommodel.

Hierna is hij gedurende twee jaar actief geweest op het gebied van de software ontwikkeling en werd tevens een bedrijfsinformatica opleiding gevolgd. Op 1 juli 1988 trad hij in dienst bij de toen 1 jaar jonge vakgroep wiskunde van de Rijksuniversiteit Limburg, waar hij onder andere software pakketten ontwikkelde ter ondersteuning van een aantal lopende projecten.

Naast deze activiteiten zette hij in 1989 zijn wiskundig onderzoek voort onder begeleiding van Prof.Dr.Ir.Drs. O.J. Vrieze. De resultaten hiervan zijn beschreven in dit proefschrift. 